\title{
Iman Attia,
}

Ozan Zakariya Keskinkılıç, Büşra Okcu

\section{Muslimisch- sein im Sicherheits- diskurs}

Eine rekonstruktive Studie über den Umgang mit dem Bedrohungsszenario

[transcript] Kultur und soziale Praxis 
Iman Attia, Ozan Zakariya Keskinkılıç, Büşra Okcu Muslimischsein im Sicherheitsdiskurs 
Iman Attia (Dr. phil.) ist Professorin an der Alice Salomon Hochschule Berlin. Sie lehrt und forscht zur Relationalität von Rassismen und ihren Intersektionen mit anderen gesellschaftlichen Machtverhältnissen. Ihr Schwerpunkt liegt neben Erinnerungsarbeit und globalhistorischen Narrativen vor allem auf antimuslimischem Rassismus.

Ozan Zakariya Keskinkılıç ist Politikwissenschaftler und Rassismusforscher. Seine Forschungsschwerpunkte sind u.a. (antimuslimischer) Rassismus, Antisemitismus, Orientalismus und Empowerment.

Büşra Okcu ist Praxisforscherin der Sozialen Arbeit und Pädagogik. Ihre Schwerpunkte liegen auf rekonstruktiven Methoden aus rassismuskritischer Perspektive, antimuslimischem Rassismus und Beratungsprozessen in der muslimischen Sozialen Arbeit. 
Iman Attia, Ozan Zakariya Keskinkılıç, Büşra Okcu

\section{Muslimischsein im Sicherheitsdiskurs}

Eine rekonstruktive Studie über den Umgang mit dem Bedrohungsszenario

Unter Mitarbeit von Ouassima Laabich-Mansour

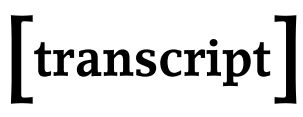


Die vorliegende Publikation wurde aus Mitteln des Bundesministeriums für Bildung und Forschung als Teilprojekt »Gouvernementalität« des Verbundprojekts »Das Bedrohungsszenario des sislamistischen Terrorismus aus den Perspektiven von Politik, Medien und muslimischen Communities - eine empirische Studie« unter dem Kennzeichen o1UG1830BX gefördert.

GEFŌRDERT VOM

拸1 $\begin{aligned} & \text { Bundesministerium } \\ & \text { für Bildung } \\ & \text { und Forschung }\end{aligned}$

\section{Bibliografische Information der Deutschen Nationalbibliothek}

Die Deutsche Nationalbibliothek verzeichnet diese Publikation in der Deutschen Nationalbibliografie; detaillierte bibliografische Daten sind im Internet über http://dnb.d-nb.de abrufbar.

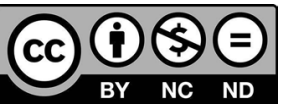

Dieses Werk ist lizenziert unter der Creative Commons Attribution-NonCommercial-NoDerivs 4.0 Lizenz (BY-NC-ND). Diese Lizenz erlaubt die private Nutzung, gestattet aber keine Bearbeitung und keine kommerzielle Nutzung. Weitere Informationen finden Sie unter https://creativecommons.org/licenses/by-nc-nd/4.o/deed.de

Um Genehmigungen für Adaptionen, Übersetzungen, Derivate oder Wiederverwendung zu kommerziellen Zwecken einzuholen, wenden Sie sich bitte an rights@transcript-publishing.com

Die Bedingungen der Creative-Commons-Lizenz gelten nur für Originalmaterial. Die Wiederverwendung von Material aus anderen Quellen (gekennzeichnet mit Quellenangabe) wie z.B. Schaubilder, Abbildungen, Fotos und Textauszüge erfordert ggf. weitere Nutzungsgenehmigungen durch den jeweiligen Rechteinhaber.

\section{() 2021 transcript Verlag, Bielefeld}

Umschlaggestaltung: Kordula Röckenhaus, Bielefeld

Umschlagcredit: »WE MUST NOT BE EXTREME« 2017, Artwork by: Faisal Hussain, www.suspectobjects.com, Photograph: David Rowan

Lektorat: Julia Jürgens, Berlin

Korrektorat: Luisa Bott, Bielefeld

Druck: Majuskel Medienproduktion $\mathrm{GmbH}$, Wetzlar

Print-ISBN 978-3-8376-5711-1

PDF-ISBN 978-3-8394-5711-5

https://doi.org/10.14361/9783839457115

Gedruckt auf alterungsbeständigem Papier mit chlorfrei gebleichtem Zellstoff. Besuchen Sie uns im Internet: https://www.transcript-verlag.de Unsere aktuelle Vorschau finden Sie unter www.transcript-verlag.de/vorschau-download 


\section{Inhalt}

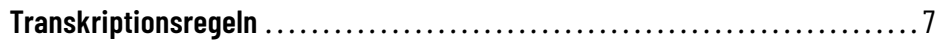

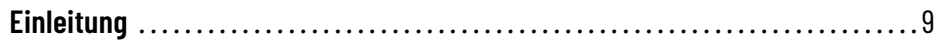

Der antimuslimische Rassismus und sein Sicherheitsdiskurs ........... 13

Traditionen des Sicherheitsdiskurses ............................ 13

Antimuslimischer Rassismus als Gesellschafts- und Erfahrungshintergrund.... 17

Die Adressierung von `Muslim:innen als Sicherheitsproblem ............... 19

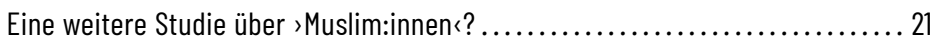

Theoretische Überlegungen zu Fremd- und Selbstführung.............. 29

Gouvernementalität, Biopolitik und Rassismus ...................... 30

Freiheit und (Selbst-)Führung im (antimuslimischen) Rassismus .......... 35

Subjekt, Widerstand und Handlungsmacht ....................... 38

Schlussfolgerung......................................... 46

Rekonstruktive Forschungsmethoden rassismuskritisch perspektiviert ... 49

Sample, Interviewführung und das Problem mit den Typologien............. 51

Nutzen und Grenzen rekonstruktiver Methoden ...................... 56

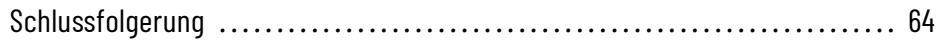

Ver_Handlungen ......................................... 67

Inter_Sektionen .......................................... 69

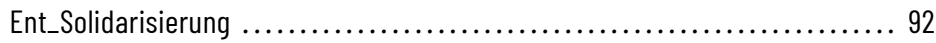

Ver_Antworten ........................................... 116 


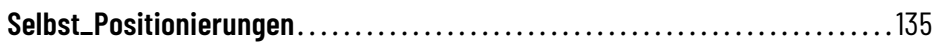

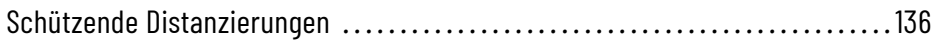

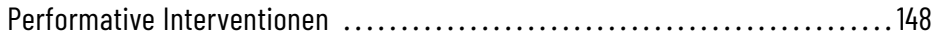

Globalhistorische Verflechtungen ............................ 159

Zusammenfassung und Fazit .................................. 169

Zusammenfassung der Rekonstruktionsergebnisse .................... 171

Islambezogene Gouvernementalität: (Selbst-)Führung und Widerstand ........ 175

Schlussfolgerung und Ausblick .................................. 178

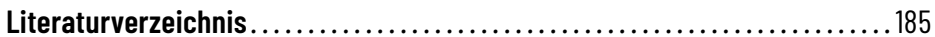




\section{Transkriptionsregeln}

\begin{tabular}{|l|l|}
\hline $\mathrm{L}$ & Beginn einer Überlappung \\
\hline $\mathrm{J}$ & Ende einer Überlappung \\
\hline$()$. & Pause bis zu einer Sekunde \\
\hline$(2)$ & Anzahl der Sekunden einer Pause \\
\hline Nein & Betonung \\
\hline LAUT & laut, akzentuiert (auch Silben, z.B. wOllen) \\
\hline nei::n & $\begin{array}{l}\text { Dehnung, wobei die Anzahl der Doppelpunkte aufdie Länge } \\
\text { des gedehnten Wortes verweist }\end{array}$ \\
\hline oja ${ }^{\circ}$ & sehr leise gesprochenes Wort \\
\hline @ & kurzes Lachen unter einer Sekunde \\
\hline @(.)@ & kurzes Lachen mit Angabe der Sekunden in der Klammer \\
\hline @nicht@ & lachend gesprochen \\
\hline Büch- & Abbruch eines Wortes \\
\hline ach=nee & $\begin{array}{l}\text { Stottern, Wiederholung, schnell gesprochen, Wortschlei- } \\
\text { fung }\end{array}$ \\
\hline (naja) & schwer verständliches Wort \\
\hline 0 & unverständliches Wort \\
\hline$((a t m e t ~ a u s))$ & Beschreibung nicht-sprachlicher Vorgänge \\
\hline
\end{tabular}





\section{Einleitung}

Das vorliegende Buch ist das Ergebnis eines qualitativen Forschungsprojekts. Das Teilprojekt Gouvernementalität des BMBF-geförderten Verbundprojekts Bedrohungsszenario des islamistischen Terrorismus aus den Perspektiven von Medien, Politik und muslimischen Communities war an der Alice Salomon Hochschule Berlin angesiedelt. Von Februar 2018 bis Januar 2021 nahmen wir die Perspektiven muslimischer und als muslimisch markierter Akteur:innen zum Ausgangspunkt, um ihre Erfahrungen und Deutungen zum Sicherheitsdiskurs in Deutschland und ihre Strategien im Umgang damit zu erfragen bzw. zu rekonstruieren. Der hegemoniale Sicherheitsdiskurs konstruiert >Muslim:innen aufgrund ihrer (zugeschriebenen) Religion, Kultur und/oder Herkunft als Bedrohung und nimmt sie ins Visier strafrechtlicher Maßnahmen, interdisziplinärer Forschung sowie pädagogischer und politischer Programme. (Als) Muslim:innen (Markierte) werden beobachtet, vermessen und diszipliniert, der Sicherheitsdiskurs zielt auf ihr Verhalten, ihre Wahrnehmung und ihre Bewegung im öffentlichen Raum, mit dem Ziel, sie (in-)direkt unter der Figur der >deutschen Leitkultur zu führen. Vor diesem Hintergrund arbeiten wir heraus, wie muslimische Akteur:innen Deutungs- und Handlungsmacht über ihr privates und gesellschaftliches Leben sowie ihre aktivistische und berufliche Arbeit (wieder-)gewinnen, wie sie das Verhältnis von Zustimmung zu und Kritik an ihrer Adressierung austarieren und welche Freiräume sie sich schaffen, um die Diskursivierung des muslimischen Subjekts nicht (gänzlich) anderen zu überlassen und ihre Interessen und Bedürfnisse $\mathrm{zu}$ verwirklichen. 
>Muslim:innen gelten nicht erst seit 9/11 als bedrohlich (und) fremd: Das Bedrohungsszenario, das Muslim:innen und als solche Markierte als Sicherheitsrisiko adressiert, stellt einen der Diskursstränge des antimuslimischen Rassismus dar. Darüber ist im ersten Kapitel mehr zu lesen. Das zweite Kapitel dient der theoretischen Grundlegung: Mit dem Begriff der Gouvernementalität bietet Foucault ein für die vorliegende Forschungsfrage nützliches theoretisches Grundgerüst an, um das Kräfteverhältnis von Unterwerfung und Widerstand, den Prozess der Subjektwerdung und damit einhergehend das Zusammenspiel der Regierung durch andere mit Selbsttechnologien in den Mittelpunkt der Untersuchung zu rücken. Anschließend stellen wir die zur Erhebung und Auswertung eingesetzten Methoden vor. Die Dokumentarische Methode und die Objektive Hermeneutik dienen uns als Werkzeuge, die wir rassismuskritisch modifizieren. Vor dem Hintergrund unserer theoretischen und methodologischen Auseinandersetzungen führten und rekonstruierten wir drei Gruppendiskussionen und zehn Einzelinterviews und entwickelten induktiv sechs Spannungsfelder, die in den Kapiteln Ver_Handlungen und Selbst_Positionierungen vorgestellt werden. Die Studie endet mit einer Zusammenfassung der empirischen Befunde sowie methodischer und theoretischer Überlegungen.

Wir danken herzlich allen 24 Gesprächspartner:innen, die uns offen und bereitwillig ihr Vertrauen schenkten und ihre Gedanken mitteilten. Sie haben das vorliegende Buch erst möglich gemacht. Die reflektierten und fundierten Überlegungen, die Intensität der Debatten und die Differenziertheit der Ausführungen haben uns so manche Analyse erleichtert und viele interessante Erkenntnisse ermöglicht. Gleichzeitig wurden die doch häufig durch Repression, Begrenzung und Entrechtung hervorgerufenen Gefühle der Ausweglosigkeit und der Bedrückung von Humor, Witz und Ironie und auch von Inspiration, Utopie und Einfallsreichtum unterbrochen, sodass die Arbeit an den Transkripten oft genug zu einem vergnüglichen und anregenden Erlebnis wurde. Wir danken Euch und Ihnen allen sehr dafür!

Ouassima Laabich-Mansour hat als studentische Mitarbeiterin die Arbeit im Forschungsprojekt von Beginn an bereichert. Ihre Erfahrungen, Perspektiven und Reflexionen haben zum Gelingen beigetragen. 
Dafür gebührt Dir unsere Anerkennung. Deine Herzlichkeit und dein Lachen haben wiederum vieles leichter gemacht.

Danken möchten wir auch Alexander Fahim, Latifa Hahn, Katja Kinder, Malika Mansouri, Kofi Ohene-Dokyi, Rümeysa Şenel und Constantin Wagner, die an Interpretationsgruppen teilnahmen. Unser besonderer Dank geht an Schirin Amir-Moazami, Malika Mansouri und Constantin Wagner, die Teile des Manuskripts vorab gelesen und uns wichtige Rückmeldungen gegeben haben. Julia Jürgens besorgte das Lektorat, auch dafür vielen Dank. 



\section{Der antimuslimische Rassismus und sein Sicherheitsdiskurs}

\section{Traditionen des Sicherheitsdiskurses}

Es greift zu kurz, 9/11 als Kristallisationspunkt des antimuslimischen Rassismus in den USA, Europa, Deutschland und anderen Teilen der Welt zu setzen, wie dies häufig geschieht. Anders als es das mediale und politische Sprechen über Islam, Migration und Integration oder auch einzelne Studien aus der Vorurteilsforschung zu Islamophobie suggerieren, hat die Problematisierung der Minderheit und ihre Diskursivierung als bedrohlich und fremd eine lange Tradition.

Gegenwärtige Maßnahmen zur inneren und äußeren Sicherheit, die sich in spezifischer Weise gegen und an (als) Muslim:innen (Markierte) richten, reichen bis ins späte 15. bzw. frühe 16. Jahrhundert in Südeuropa zurück. Auf der Iberischen Halbinsel etwa müssen die über Jahrhunderte ansässigen Bevölkerungsgruppen muslimischen Glaubens und arabischer oder amazighischer Sprachen nach der Machtübernahme durch katholische, spanische und portugiesische Herrscher:innen zunächst verschiedene restriktive Maßnahmen über sich ergehen lassen, die sie in der Ausübung ihrer Religion und an der Entfaltung ihrer Kulturen hindern: Sie werden zur Konversion gezwungen oder vertrieben und schließlich nach Nordafrika deportiert. ${ }^{1}$ Die Idee der neuen, christlichen Herrscher:innen, die Einheit der Nation durch religiöse Reinheit zu erreichen, koppelt die Loyalität 
gegenüber der Nation an die Religionszugehörigkeit, die wiederum genealogisch hergeleitet wird. Der eigenen Logik und Politik zufolge kann die muslimisch-arabisch-amazighische Bevölkerung, >Mauren` oder >Moriscos ～genannt, nicht loyal sein und wird deswegen als gefährlich behandelt, beobachtet, reglementiert, sanktioniert und schließlich vertrieben und deportiert. Die über mehrere Jahrhunderte andauernde Koexistenz der multilingualen und multireligiösen Gesellschaft, die in gemeinsamen Arbeiten philosophische und naturwissenschaftliche Höhenflüge erreicht, nimmt ein gewaltsames Ende. Der vormoderne Rassismus erhält seine Konturen. ${ }^{2}$

Im Zuge des europäischen Kolonialismus verändert sich die Wahrnehmung und Darstellung der >Mohammedaner:innen $<$ im >Orient $<$ und in >Afrika<, die Deutung ihrer Gefährlichkeit erfährt eine neue Variante und geht abermals mit ihrer Rassifizierung einher. Um die koloniale Herrschaft, Gewalt, Ausbeutung, Unterdrückung und Herabwürdigung $\mathrm{zu}$ rechtfertigen, werden die kolonialen >Untertanen und unzivilisiert diskursiviert, der Widerstand dagegen als Aufstand und Gewalt diffamiert. ${ }^{3}$ Die Ver-anderung und die Gewalt sind geblieben, ihre Begründung und ihre Formen verändern sich, wie Fanon argumentiert:

»Der vulgäre, primitive, gewöhnliche Rassismus gab vor, in der Biologie die materielle Basis der Doktrine zu finden, als sich die Bibel als unzureichend erwiesen hatte. [...] Nichtsdestoweniger feiern die alten Äußerungen hier und dort Wiederauferstehung. [...] Der Rassismus, der sich rational, individuell, genotypisch und phänotypisch determiniert gibt, verwandelt sich in einen kulturellen Rassismus. Das Objekt des Rassismus ist nicht länger der einzelne Mensch, sondern eine bestimmte Existenz-Form. [...] Die >westlichen Werte treffen sich seltsamerweise wieder mit dem so berühmten Aufruf zum Kampf des >Kreuzes gegen den Halbmond . $^{4}$

\footnotetext{
$2 \quad$ Hering Torres 2006.

3 Fanon 1966, 1969; Said 1978.

4 Fanon 1969: 39f.
} 
Fanons und Saids Studien zur kolonialen Gewalt und zum Orientalismus nehmen französische und englische Diskurse und Politiken in den Blick. Auch im deutschen Kolonialismus bis hin zum Nationalsozialismus spielt neben der biologischen und kulturellen Orientalisierung der Muslim:innen ihre politische Beherrschung und Instrumentalisierung eine Rolle. ${ }^{5}$ Die Frage danach, welche Gefahr von ihnen ausgeht, bezieht hier, ähnlich wie in anderen europäischen Kontexten, sowohl vormoderne als auch moderne Argumentationslinien ein ${ }^{6}$, die in aktuelle Diskurse hineinragen. Sie bringen ein spezifisches >Wissen Muslim:innen hervor ${ }^{7}$ und verdichten sich in einem neuen antimuslimischen Rassismus. ${ }^{8}$ Darin kommen dem Bedrohungsszenario und dem Sicherheitsdiskurs wieder prominente Bedeutungen $\mathrm{zu}->$ Muslim:innen werden als innere und als äußere Gefahr diskursiviert. ${ }^{9}$

Der aktuelle antimuslimische Rassismus nimmt sowohl auf den vormodernen als auch auf den modernen Rassismus Bezug, sodass einige ältere Topoi erstaunlich aktuell erscheinen - oder auch gegenwärtige, als menschenrechtliche >Islamkritik^präsentierte Äußerungen auffallend mittelalterlich. Das Wechselspiel von Religion, Kultur, Geschlecht und Sexualität etwa blickt auf eine lange Geschichte zurück ${ }^{10}$, aktuelle Analysen $\mathrm{zu}$ Homonationalismus und Femonationalismus ${ }^{11}$ können auf Vorarbeiten zum »kolonialen Feminismus « ${ }^{12}$ zurückgreifen.

Die Vorstellung einer Gefahr, die von Muslim:innen - und auch Juden:Jüdinnen - als >fünfter Kolonne Nation einer äußeren Macht zuarbeitet, finden wir ausgeprägt im 16., im ausgehenden 20. sowie im beginnenden 21. Jahrhundert wieder,

Attia 2007; Berman 1996; Fuhrmann 2006; Keskinkılıç 2019a; Motadel 2017; Wagenhofer 2010.

6 Bellmann 2011; Höfert 2003; Katzer 2008.

7 Amir-Moazami 2018; für Europa: Johansen/Spielhaus 2012.

8 Attia 2009; für Europa und USA: Attia/Keskinkılıç 2016.

9 Benz 2012.

10 Kabbani 1993; Pinn/Wehner 1995; Shooman 2014; Yeğenoğlu 1998.

11 Zum Homonationalismus Puar 2007; zum Femonationalismus Farris 2011.

12 Ahmed 1992: 151. 
während die angebliche Gefahr, die von der vermeintlichen Rückständigkeit und dem Widerstand der Muslim:innen (und der anderen ausgebeuteten Völker) gegen Kolonialismus und die >Neue Weltordnung ausgeht, das 18. und 19. mit dem 21. Jahrhundert verbindet. Die Transformation spiegelt die Entwicklung des Rassismus: Während der vormoderne Rassismus $>$ Rassen im Plural als getrennte Entitäten konzipiert, führt der moderne Rassismus die vormals getrennten $>$ Rassen zusammen - die Differenz wird nun linear in der Entwicklung der Menschheitsrasse, >Rasse $<$ im Singular also, angeordnet. Insofern verwundert es nicht, wenn im vormodernen Rassismus die Gefahr auch dann als äußere wahrgenommen wird, wenn diejenigen, von denen die Gefahr ausgehen soll, im Inneren leben (Illoyalität), während die Gefahr im modernen Rassismus als universalistische, weil den Fortschritt der Menschheit bzw. die >Rassenhygiene zipiert wird (Minderwertigkeit). In jeweils angepasster Sprache und Bezügen finden wir beide Diskursstränge in aktuellen Rassismen, auch im antimuslimischen, wieder. >Muslim:innen sind demzufolge sowohl gefährlich, »weil sie wirklich im Weltmaßstab operieren " $^{13}$ und »an der Übernahme der Macht « ${ }^{14}$ arbeiten, als auch, weil sie aufgrund ihrer »enorme[n] Fruchtbarkeit [...] auf lange Sicht eine Bedrohung für das kulturelle und zivilisatorische Gleichgewicht [und einen] Rückschritt « ${ }^{15}$ darstellen. Insofern verbinden sich im antimuslimischen Rassismus Argumentationsmuster des Antisemitismus mit jenen des kolonialen Rassismus, weisen aber auch Unterschiede zu beiden auf und stellen daher eine spezifische Form von Rassismus dar. ${ }^{16}$

Der kurze Abriss zeigt, dass der Sicherheitsdiskurs nicht neu ist und die Vermutung, dass er auf die Gewalt reagiert, die von (als) Muslim:innen (Markierten) ausgeht, zu kurz greift. ${ }^{17}$

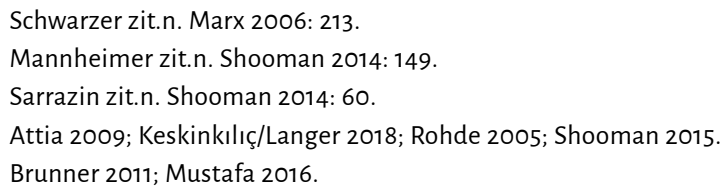




\section{Antimuslimischer Rassismus als Gesellschafts- und Erfahrungshintergrund}

Die Stereotypisierung, Diskriminierung und Verfolgung von (als) Muslim:innen (Markierten) damit zu entschuldigen, dass es nicht die Falschen trifft, angesichts von Terrorismus und Radikalisierung, Illoyalität und Fremdheit, Rückständigkeit und Traditionalismus, verkennt die historischen und gesellschaftlichen Zusammenhänge und bedient sich genau jener Argumentationen, die sie zu kritisieren vorgibt. AlAzmeh vergleicht Die Islamisierung des Islam, so der Titel seines Buches, in europäischen Diskursen mit der "Islamisierung des Islam ${ }^{18}$ durch islamistische Ideologien. Demnach verbindet islamistische und antimuslimische Argumentationen eine Komplizenschaft, die auf der naturalisierenden Kulturalisierung von Differenz beruht und in Rassismus verstrickt ist. Muslimische Gemeinschaften werden in beiden Varianten überislamisiert und »aus konkreten sozialen, historischen, politischen, ideologischen Gebilden mit bestimmten Tendenzen und Perspektiven ${ }^{19} \mathrm{zu}$ einem ahistorischen, essentiellen Islam umgedeutet. Weil Religion als Kultur und beides als unveränderlich und statisch verhandelt wird, spricht Al-Azmeh von Rassismus. Auch Said hatte den kolonialen Orientalismus des 18. und 19. Jahrhunderts als Rassismus theoretisiert. ${ }^{20}$ Aktuelle Fachdebatten stimmen mit dieser Einordnung überein und sprechen von antimuslimischem Rassismus. ${ }^{21}$

Der antimuslimische Rassismus hat Folgen, die über die Wahrnehmung von Muslim:innen als fremd und bedrohlich und die Geringschätzung des Islams und der Muslim:innen hinausgehen. ${ }^{22}$ Im Bedrohungsszenario des sislamistischen Terrorismus` werden (als) Muslim:innen (Markierte) ihrer tatsächlichen oder zugeschriebenen Religion, Kultur und Herkunft nach als spezifische Problemgruppe ins

\footnotetext{
18 Al-Azmeh 1996: 21.

19 Ebd.: 7.

20 Said 1978, 1985.

21 Attia/Keskinkılıç 2016.

22 Hafez/Schmidt 2014.
} 
öffentliche, politische, wissenschaftliche und pädagogische Visier genommen und unter Aufsicht gestellt. Das Bedrohungsszenario mündet in Politiken der Kriminalisierung, Verdächtigung und Überwachung von (als) Muslim:innen (Markierten) als potentielle »Gefährder « ${ }^{23}$. Und das, obwohl »die Geschichte des modernen Terrorismus keine Geschichte islamistischer Gruppierungen« ist und »andere Formen staatlicher wie nicht staatlicher Gewalt ein rechnerisch größeres Gefährdungspotenzial für die innere wie auch internationale Sicherheit $\aleph^{24}$ besitzen. Gleichzeitig wird gegen Muslim:innen gerichtete rassistische und extrem rechte Gewalt individualisiert und marginalisiert ${ }^{25}$ sowie Kritik am antimuslimischen Rassismus verharmlost und diskreditiert. $^{26}$

(Als) Muslim:innen (Markierte) werden mit dem Stigma des bedrohlich Fremden belegt, misstrauisch beobachtet und kommentiert, sie werden der Integrationsverweigerung oder der Integrationsunfähigkeit sowie der Unterwanderung und Islamisierung bezichtigt, sodass ihre Diskriminierung nicht nur als gerechtfertigt, sondern bisweilen gar als geboten erscheint. >Muslim:innen bleiben von hegemonialen Islamdebatten und der damit zusammenhängenden Anrufung als Sicherheitsbedrohung nicht unberührt: Sie sehen sich mit alltäglichen Anfeindungen auf offener Straße konfrontiert, erfahren institutionelle Diskriminierung in Bildungseinrichtungen, im Beruf, auf dem Wohnungsmarkt und im Gesundheitssystem ${ }^{27}$ und müssen sich - ob sie wollen oder nicht - mit jenen Themen auseinandersetzen, die im hegemonialen Islamdiskurs als 'muslimisch und deswegen als problematisch erscheinen. Sie werden dazu angehalten, sich für die Taten anderer $>$ Muslim:innen zu verantworten, von `bösen Muslim:innen $\mathrm{zu}$ distanzieren und sich unaufhörlich als integrierte, aufgeklärte Bürger:innen zu beweisen.

\footnotetext{
23 Bäcker 2020; Denkowski 2014; Graebsch 2019; Jukschat/Leimbach 2019.

24 Bock 2017: 250.

25 Denkowski 2014; Jukschat/Leimbach 2019.

26 Bartel/Liebscher/Remus 2017; Cremer 2017; Naguib 2013.

27 Bayoumi 2009; Elahi/Khan 2017; Lewicki 2016; Mühe 2019.
} 
Ein möglicher Umgang damit ist, die religiösisierende und kulturalisierende Sicht auf Gewalt, die von >Muslim:innen risch $\mathrm{zu}$ kontextualisieren ${ }^{28}$ oder auch muslimische Kritik daran europäischen Leser:innen zugänglich zu machen. ${ }^{29}$ In der vorliegenden Studie werden wir weitere Deutungen und Strategien im Umgang mit dem Bedrohungsszenario und mit der Adressierung von >Muslim:innen Sicherheitsrisiko rekonstruieren.

\section{Die Adressierung von ıMslim:innen als Sicherheitsproblem}

Die folgenden Ausführungen sind das Ergebnis eines Forschungsprojekts, das (als) Muslim:innen (Markierte) fragt, wie sie den Sicherheitsdiskurs wahrnehmen, welche Bedeutung er für sie hat und wie sie damit umgehen. In Einzelinterviews befragt und zu Gruppendiskussionen eingeladen haben wir Akteur:innen, die in Verbänden, Communities oder Gemeinden aktiv sind und die sich selbst als muslimisch verorten oder als solche angesprochen werden. Damit knüpft das Forschungsprojekt an eine Praxis an, die >Muslim:innen nicht nur als besondere Gruppe ver-andert, sondern im Kontext des Sicherheitsdiskurses anspricht.

Belege für die Adressierung von (als) Muslim:innen (Markierten) als Sicherheitsproblem lassen sich in Praxis reflektierender und erforschender Literatur finden. Qasem ${ }^{30}$ schildert, dass er von einer Schule gebeten wird, einen Workshop durchzuführen. Der Auftrag wird damit begründet, dass einige >muslimischeく Schüler:innen »auffälliges Verhalten« zeigen, ein Schüler soll »auf dem Schulhof predige[n]«. »Ich selber beobachtete in meinen Gesprächen während der Workshops jedoch keinerlei besonderen religiösen Eifer bei diesem Schüler, sondern nahm seine mitunter tatsächlich lautstarken Beschwerden über Rassismus vielmehr als aufrichtige Unmutsäußerungen wahr«, so Qasem. 
Dieses Beispiel steht paradigmatisch für viele seiner Erfahrungen im Zusammenhang mit der Islamismusprävention: "Von Rassismus betroffene Jugendliche - und unter ihnen vor allem diejenigen, die diese Erfahrung auch klar als Erfahrung von Rassismus benennen - werden so automatisch als potenziell radikalisierungsgefährdet gelesen«.

Eine Studie von Karakaşoğlu und Wojciechowicz bestätigt diese Schilderung. ${ }^{31}$ Die Autorinnen zitieren einen Schulleiter, der sich gegen eine Referendarin mit Hijab »energisch zur Wehr gesetzt [hat] «, weil er meint, dass »das Kopftuch die demokratisch strukturierte Ordnung der Schule gefährde[t]«. Ein anderer Schulleiter unterstellt eine Praktikantin mit Hijab seiner direkten Aufsicht (im Unterschied zu den anderen Praktikant:innen, die von der Ausbildungskoordinatorin betreut werden) und versetzt sie wöchentlich in eine andere Klasse, wo sie in der letzten Reihe sitzen muss, weil der Schulleiter nicht will, dass die Schüler:innen sie als künftige Lehrerin anerkennen. Die Erfahrung junger Menschen in (außer-)schulischen Bildungskontexten, als Bedrohung und Sicherheitsrisiko adressiert zu werden, reiht sich in ihre allgemeinen Rassismuserfahrungen ein $^{32}$ und betrifft freilich nicht nur Jugendliche. Im Rahmen einer Studie führen Jukschat und Leimbach Interviews im Justizvollzug durch. Sie berichten, wie ihnen "mehrheitlich vermeintlich >radikalisierte Interviewpartner vermittelt wurden, die, wie sich im biografischen Interview und der rekonstruktiven Analyse zeigte, weder demokratiefeindliche Positionen vertraten noch mit islamistischen Akteur:innen sympathisierten «. ${ }^{33}$ In einem Fall stellt sich heraus, dass die als Islamist vorgestellte Person ein Aktivist der PKK ist und im anderen, dass es sich um einen kurdischen Jesiden handelt.

Quantitative Erhebungen und Meinungsumfragen, die (als) Muslim:innen (Markierte) befragen und erforschen, und von Wissenschaft, Politik, Verwaltung, Medien und NGOs in Auftrag gegeben werden, gewinnen in den letzten Jahrzehnten vermehrt an Bedeutung. Sie bilden

31 Karakaşoğlu/Wojciechowicz 2017: 517-518.

32 Mühe 2019; Scharathow 2014, 2017.

33 Jukschat/Leimbach 2019: 15. 
den Korpus einer Untersuchung von Johansen und Spielhaus ${ }^{34}$. Die Autorinnen analysieren Studien, die über zehn Jahre in sieben west- und nordeuropäischen Staaten durchgeführt wurden. Sie fragen danach, welche Vorstellungen darin über Muslim:innen zum Ausdruck kommen: Wer gilt als muslimisch, wie wird die Stichprobe der $>$ Muslim:innen operationalisiert und wie kommt sie zustande? Wer wird als Muslim:in befragt - und wer nicht, welche Themenfelder werden angesprochen, welche Fragen gestellt - und welche nicht, welches Erkenntnisziel liegt den Befragungen zugrunde und wie werden die Ergebnisse präsentiert? Die Befragungen, so ein Ergebnis von Johansen und Spielhaus, greifen auf gesellschaftlich verfügbares Wissen über Muslim:innen zurück und sind ihrerseits an der Produktion von Wissen über Muslim:innen beteiligt, und zwar im Zuge des gesamten Forschungsprozesses, vom Entwurf des Forschungsdesigns und der Zusammenstellung des Samples, über den Erhebungs- und Auswertungsprozess bis hin zur Präsentation der Ergebnisse.

Die wenigen Beispiele zeigen, dass es auch kritische Auseinandersetzungen mit der einseitigen und unterkomplexen Erforschung von >Muslim:innen gibt sowie Studien, die Muslim:innen dazu einladen, eigensinnige Perspektiven einzunehmen, und Abhandlungen, die die fachlichen Kompetenzen von Muslim:innen ernst nehmen. ${ }^{35}$

\section{Eine weitere Studie über ’Muslim:innen`?}

Unsere Studie folgt der Logik, >Muslim:innen`zum Untersuchungsgegenstand $\mathrm{zu}$ nehmen und sie $\mathrm{zu}$ einem problematischen Sachverhalt zu befragen. Da wir die Effekte der Muslimisierung untersuchen wollen, müssen wir >Muslim:innen $<$ befragen. Wir müssen dem Konstruktionsprozess des >muslimischen Sicherheitsproblems` nachspüren, um seine Funktions- und Wirkungsweisen zu verstehen und ihn »kontra-

34 Johansen/Spielhaus 2012, 2018.

35 Spielhaus 2011; Bassiouni 2014. 
punktisch $\ll^{36}$, also sowohl in der hegemonialen Bedeutung und deren Vereindeutigungen und Auslassungen als auch den Widerständen dagegen, lesen zu können. Die Diskursivierung von >Muslim:innen`als Sicherheitsproblem ist ein gesellschaftlicher Rassifizierungsprozess ${ }^{37} \mathrm{mit}$ realen Folgen für (als) Muslim:innen (Markierte). Von einer Konstruktion als einem Prozess der Rassifizierung zu sprechen, bedeutet freilich nicht, dass es keine Menschen gibt, die sich als Muslim:innen verstehen; vielmehr ist damit die naturalisierende Homogenisierung von Menschen gemeint, die sie aufgrund ihrer (vermeintlichen oder tatsächlichen) Herkunft zu kulturell Fremden erklärt, ihre Fremdheit mit der (vermeintlichen oder tatsächlichen) Religionszugehörigkeit begründet und damit die Herabsetzung und Entwürdigung der so zu >Muslim:innen rassifizierten Menschen rechtfertigt.

Der rassifizierende Konstruktionsprozess hat unter anderem zur Folge, dass sich >Muslim:innen $`$ mit Rassismus auf die ein oder andere Weise beschäftigen müssen, auch wenn sie es nicht wollen. Da Rassismus nicht verschwindet, wenn sich Menschen entscheiden, ihn zu ignorieren, muss diese Entscheidung immer wieder neu getroffen werden; so findet die Beschäftigung mit Rassismus also auch dann kein Ende, wenn ihr eine rassifizierte Person aus dem Weg zu gehen versucht: Sie wird weiterhin als $>$ Muslim:in $<$ adressiert und muss sich aktiv dazu oder auch dagegen verhalten. Rassismus zu ignorieren, ist also keine Option, jedenfalls nicht für Menschen, die rassifiziert werden, und nicht in kritischer Absicht. Um Rassismus der Kritik zugänglich zu machen mit dem Ziel, ihn oder zumindest seine Folgen zu minimieren, muss er thematisiert werden.

Eine der Möglichkeiten, Rassismus zu identifizieren, besteht darin, seine Effekte $\mathrm{zu}$ studieren. Insbesondere nicht-intendierter, institutionalisierter, wohlmeinender, paternalistischer Rassismus kann nur schwerlich an den Motiven erkannt werden. Handlungsroutinen etwa können problematisch sein, wenn ihnen eine spezifische Norm (weiß,

36 Said 1994: 51

37 Attia 2009; Shooman 2014. 
christlich, säkular) zugrunde liegt, die partikular ist, aber universalisiert wird; Personen müssen sich nichts weiter dabei denken, wenn sie wie üblich handeln, und dennoch kann der Effekt ausschließen und dem Ausschluss eine rassistische Konstruktion zugrunde liegen. ${ }^{38}$ Grenzüberschreitendes Verhalten, das damit begründet wird, nur das Beste im Sinn $\mathrm{zu}$ haben und helfen $\mathrm{zu}$ wollen, kann in rassistische Diskurse verstrickt sein, ohne dass die so handelnde Person ein rassistisches Motiv benennen könnte. Und doch haben diese Formen von Rassismus Herabsetzung und Verletzung, Barrieren und Ungleichheit, Wut und Rückzug zur Folge.

Antimuslimischer Rassismus (be-)trifft Menschen, die als >Muslim:innen angesprochen und ausgeschlossen, bevormundet und bemitleidet, zurückgewiesen und behindert, beschimpft und beschuldigt werden, der Sicherheitsdiskurs (be-)trifft (als) Muslim:innen (Markierte). Insofern müssen wir >Muslim:innen befragen, wenn wir rassismuskritische Forschung betreiben wollen, auch auf die Gefahr hin, die Konstruktion der Gruppe der >Muslim:innen - jedenfalls vorübergehend - dadurch zu affirmieren, dass wir unsere Gesprächspartner:innen als solche anrufen.

Johansen und Spielhaus kommen in ihrer Analyse zu dem Schluss, dass in der überwiegenden Mehrzahl der Befragungen bereits die Fragestellung und die Auswahl der Gesprächspartner:innen davon ausgehen, dass Muslim:innen fremd und potentiell problematisch sind. Die Themenfelder nehmen folgende Aspekte in den Blick: »integration, belonging and identity, radicalization and security, religiosity and religious practice, political and civic participation, gender and sexuality «. ${ }^{39}$ In Fragestellungen zu Einstellungen, Werten und Praktiken werden die genannten Themenfelder derart miteinander ins Verhältnis gesetzt,

38 Das Neutralitätsgesetz etwa wurde im Zuge der Kopftuch-/Berufsverbote für Kopftuch tragende, muslimische Frauen an staatlichen Schulen erlassen. Die Begründung für das Verbot findet sich im Cesetzestext nicht wieder, seine Folgen für Hijabis sind geblieben. 
dass im Ergebnis die (vermutete) Religionszugehörigkeit von >Muslim:innen als potentielles Integrationshindernis interpretiert wird und die misslungene Integration als potentielles Sicherheitsrisiko. Über den Aspekt der Integration werden also Religion und Sicherheit miteinander verbunden. Da das Thema Integration nur Migrant:innen (und davon nur bestimmte) betriff, werden deutsche Muslim:innen ohne Migrationsbezüge aus Studien über >Muslim:innen ausgeklammert ${ }^{40}$, während Befragte, die sich trotz Adressierung als Muslim:in als atheistisch oder säkular bezeichnen, nicht aus der Stichprobe entfernt werden. ${ }^{41}$ Die Zusammenstellung des Samples und die in den Blick genommenen Fragen stellen die (vermutete) Differenz, die Religion an Kultur und Nation bindet, in den Mittelpunkt - schließlich begründet diese eine eigene Befragung von >Muslim:innen<. Wenig überraschend erscheinen >Muslim:innen dann tatsächlich als fremd bzw. werden als fremd präsentiert - alles andere wäre unspektakulär und würde keine Sonderbefragung rechtfertigen.

Auch wir überlassen den von uns interviewten Personen und Gruppen nicht die Wahl des Themas, sondern haben ein Erkenntnisinteresse. Hierzu bedienen wir uns zwar offener Erhebungsmethoden, laden unsere Gesprächspartner:innen aber zu einem spezifischen, problematisierenden Thema ein. In der vorliegenden Publikation reihen wir keine Zitate aneinander - auch das würde nicht ungefiltert Aussagen der Gesprächspartner:innen wiedergeben, sondern wäre durch uns ausgewählt und angeordnet -, sondern interpretieren die Aussagen kontrolliert und versuchen $\mathrm{zu}$ rekonstruieren: Letztlich treffen wir als Forschungsteam, das ein Forschungsdesign entwirft, Fragestellung und Methoden festlegt, Interviewpartner:innen und Diskussionsteilnehmer:innen einlädt, Passagen auswählt und interpretiert, den gesamten Prozess über Entscheidungen. Wir haben versucht, der Homogenisierung von (als) Muslim:innen (Markierten) entgegenzuwirken, indem wir das Sample der $\mathrm{zu}$ befragenden >Muslim:innen divers zusammenstellen. Der Problematisierung von >Muslim:innen

$40 \quad$ Hernández Aguilar 2018.

41 Spielhaus 2013. 
wiederum begegnen wir, indem wir diese ins Zentrum unserer Studie stellen: Wir bitten unsere Gesprächspartner:innen, über die Bedeutung des Sicherheitsdiskurses für sie zu sprechen. In Einzelinterviews und Gruppendiskussionen geben wir ihnen die Gelegenheit, ihre Wahrnehmungen und Strategien zu explizieren, ihr Muslimischsein angesichts des Bedrohungsszenarios selbst zu definieren und zu verkomplizieren, miteinander auszuhandeln und voneinander abzugrenzen, sodass sie aus der fremdbestimmten Positionierung >Muslim:in heraustreten und in Selbstpositionierungen aus pluralen, intersektionalen, ambivalenten, brüchigen Perspektiven von Muslim:innen sprechen können. Dies ist mit der Hoffnung verbunden, dass sie auch als solche gehört werden.

Die ausgewählten Auswertungsmethoden orientieren sich an einer rekonstruktiven Methodologie. Sie geht davon aus, dass die Wirklichkeit gesellschaftlich konstruiert ist und es Aufgabe der empirischen Forschung ist, "die (sinnhaften) Konstruktionen der Wirklichkeit zu rekonstruieren, welche die Akteure in und mit ihren Handlungen vollziehen $«{ }^{42}$ Wissensbestände, die Handlungen anleiten, sind meist implizit, sodass nicht einfach danach gefragt werden kann. Vielmehr müssen Handlungen, wozu auch Sprachhandlungen gehören, beobachtet und rekonstruiert werden, um die »implizite Klugheit ${ }^{43}$ sichtbar $\mathrm{zu}$ machen. Das Ziel der Rekonstruktion ist es, "soziale Verhältnisse als Sinnzusammenhänge erfassen zu können und auf diese Weise [...] einen verstehenden Nachvollzug sozialen Handelns zu ermöglichen«, unter anderem auch, weil »sich in der Art und Weise, wie die soziale Welt erfahren wird, milieu- oder generationstypische Lebensbedingungen dokumentieren « ${ }^{44}$ können.

(Als) Muslim:innen (Markierte) zu befragen bedeutet also, dass wir davon ausgehen, dass sie ein Set an sozialen Wirklichkeiten und Wissensbeständen teilen, das Rückschlüsse auf ihre Lebensumstände ermöglicht. $\mathrm{Zu}$ rekonstruieren (oder auch auf andere Weise Daten

\footnotetext{
42 Meuser 2018: 207.

43 Bohnsack zit.n. ebd.: 208.

44 Meuser 2018: 207.
} 
auszuwerten) ist daher kein technokratischer Arbeitsschritt, der von jeder beliebigen Person angewendet zum gleichen Ergebnis gelangt. Vielmehr kommt in allen Schritten des Forschungsprozesses zum Ausdruck, welches (Alltags-)Wissen die Forschenden einspeisen und zur Geltung bringen und wie sie selbst in das Thema involviert sind. Die spezifischen sozialen Wirklichkeiten, die spezifischen Wissensbestände und die besonderen Lebensumstände von >Muslim:innen können religiös, kulturell, gesellschaftlich oder historisch perspektiviert werden oder aber genau diese Perspektivierungen hinterfragt, intersektional miteinander und mit weiteren Aspekten verwoben und/oder rassismuskritisch dekonstruiert werden.

Methoden sind Werkzeuge, aber keine bloßen Techniken; sie werden $\mathrm{zu}$ einem spezifischen Erkenntniszweck eingesetzt, theoretisch begründet und (alltags-)theoretisch umgesetzt. Sie werden von gesellschaftlich positionierten Personen entwickelt und genutzt, die auf die ein oder andere Weise in rassistische Diskurse verstrickt sind, insbesondere dann, wenn die Forschenden sich unbeteiligt oder objektiv wähnen und damit ihre spezifische Position, von der aus sie forschen, nicht offen legen.

Unser Forschungsteam setzt sich aus (als) Muslim:innen (Markierten $)^{45}$ zusammen, die unterschiedliche religiöse, sprachliche, geschlechtliche, sexuelle, soziale, migrationsbiographische, disziplinäre und weitere Positionierungen und Perspektivierungen in die Arbeit einbringen. Die zu den Interpretationsgruppen eingeladenen Personen verfügen über diverse Expertisen und intersektionale Rassismuserfahrungen, sodass weitere Perspektiven hinzukommen. Die (als) Muslim:innen (Markierten), die wir für die vorliegende Studie befragen, sprechen wir - im Unterschied zum Großteil der Studien

45 Im Folgenden nutzen wir die Bezeichnungen Muslim:innen, >Muslim:innen und (als) Muslim:innen (Markierte), um (zunächst) zwischen Figur, Anrufung und Identifizierung, zwischen Selbst- und Fremdbezeichnung zu unterscheiden, die sich sowohl auf die Fokussierung auf das eine zentrale Merkmal, das Muslimischsein, bezieht als auch auf die Frage danach, ob diese Zuordnung überhaupt zutrifft. 
über Muslim:innen - nicht als Fremde und schon gar nicht als problematisch, illoyal und bedrohlich, sondern als Teil der Bevölkerung an. Und obwohl wir >muslimische Akteur:innen befragen, zielt unsere Studie nicht darauf, >Muslim:innen als Typ zu konstruieren und auch nicht verschiedene Typen >muslimischer Wirklichkeiten voneinander $\mathrm{zu}$ unterscheiden, sondern stattdessen möglichst vielfältige gouvernementale und insbesondere widerständige Umgangsstrategien mit dem Sicherheitsdiskurs zu rekonstruieren. Unsere Gesprächspartner:innen (sprach-)handeln auf unterschiedliche und häufig widersprüchliche Weisen. Sie suchen - in (selbst-)reflexiven Überlegungen und gemeinsamen Diskussionen - nach Deutungen und Positionen, nach angemessenem Verhalten und zielführenden Handlungen, sie wechseln die Richtung, sowohl im Denken als auch im Handeln. Insofern präsentieren wir als Forschungsergebnis keine Typologien, sondern Deutungen und Strategien, die um Themenspektren kreisen, welche jeweils neu verhandelt werden und zu verschiedenen, sich kreuzenden und auseinander laufenden, bisweilen ambivalenten und dann wieder klaren Positionierungen einladen. 



\section{Theoretische Überlegungen zu Fremd- und Selbstführung}

»Aber das heißt, wir sind auch Gefährder, oder wie?«, fragt Hassan M. in der dritten Gruppendiskussion besorgt in die Runde. Die Unkenntnis darüber, nach welchen Kriterien der polizeiliche Fachbegriff operiert und wie sicherheitspolitisch zwischen sguten Muslim:innen unterschieden wird, führt zu einem generellen Unbehagen unter den Diskussionsteilnehmer:innen. Sie befürchten, als Bedrohung wahrgenommen zu werden, zum Beispiel weil sie regelmäßig Moscheen besuchen oder in religiösen Vereinen aktiv sind. Von strafrechtlicher Relevanz sind diese Aspekte des alltäglichen Lebens weit entfernt, das ist allen bewusst. Gleichzeitig kommen sie nicht umhin, sich zu fragen, was passieren könnte, wenn bestimmte Orte und Akteur:innen mit Salafismus und Radikalisierung in Verbindung gebracht werden oder sie mit Gruppen und Personen assoziiert werden, die dem Spektrum des sogenannten politischen Islams zugeordnet werden. Die muslimischen Diskussionsteilnehmer:innen fühlen sich unter Druck gesetzt, ihre Loyalität und Zugehörigkeit unter Beweis zu stellen, um einer möglichen Zuordnung in eine problematische Kategorie vorzubeugen. Allein der Gedanke, als Gefährder:in, Salafist:in oder Terrorist:in wahrgenommen zu werden, führt dazu, dass sie selbst ihre Körper, ihre Sprache und ihre Kleidung, ihr Verhalten und ihre Mobilität im öffentlichen Raum daraufhin befragen, wie sie wahrgenommen werden und wie sie sich den diskursiven Anforderungen an eine sgute Bürgerschaft‘ anpassen oder entziehen. 
Die Angst davor, als gefährlich eingeordnet zu werden, kann auch dazu führen, anderen Muslim:innen und muslimischen Akteur:innen gegenüber mit Vorsicht zu begegnen. Im Alltag bedeutet das, Kontaktschuldvorwürfen ${ }^{1}$ vorzubeugen und daher Gruppen und Individuen, die möglicherweise als islamistisch eingestuft werden, zu meiden. Es bedeutet auch, sich zu überlegen, ob und gegebenenfalls in welcher Weise sie Stellung beziehen $\mathrm{zu}$ den Themen Terrorismus, Salafismus und Gewalt, die im Namen des Islams gerechtfertigt werden. ${ }^{2}$ Weder Strafe und Zwangsmaßnahmen noch direkte staatliche Kontrolle und Disziplinierung engen in dieser Sequenz die Diskussionsteilnehmer:innen im Kontext des Bedrohungsszenarios des islamistischen Terrorismus ein. Vielmehr tun dies Andeutungen und Erwartungen, Befürchtungen und exemplarische Erfahrungen Anderer, mediale und politische Diskursregeln, die sie als Muslim:innen homogenisieren und als Kollektiv als (potentielle) Gefahr adressieren. In ihren Selbstbildern und Verhaltensweisen tritt jene Machttechnologie in Erscheinung, die Foucault als Gouvernementalität ${ }^{3}$ (gouverner: regieren; mentalité: Denkweise) bezeichnet.

\section{Gouvernementalität, Biopolitik und Rassismus}

Dabei handelt es sich um eine Regierungstechnologie, »die aus den Institutionen, den Vorgängen, Analysen und Reflexionen, den Berechnungen und den Taktiken gebildete Gesamtheit, welche es erlauben, diese recht spezifische, wenn auch sehr komplexe Form der Macht auszuüben, die als Hauptzielscheibe die Bevölkerung, als wichtigste Wissensform die politische Ökonomie und als wesentliches technisches Instrument die Sicherheitsdispositive hat«. ${ }^{4}$ Im gegenwärtigen Sicherheits-

\footnotetext{
1 Schiffauer 2020.

2 Die genannte Sequenz wird im Kapitel Ver_Handlungen im Abschnitt Ent_Solidarisierung rekonstruiert.

3 Foucault 2017a.

4 Ebd.: 162.
} 
diskurs steht der nach Foucault üblichen Disziplinarmacht, die (islamistischen Terrorismus) verbietet, (Gefährder:innen) beobachtet und (islamistische Straftäter:innen) diszipliniert, eine andere Technik zur Seite, die den Rest der Muslim:innen kollektiv zur Selbstführung anhält. Foucaults Ausführungen zur Gouvernementalität sind für die vorliegende Untersuchung instruktiv, weil sie den Blick auf Sicherheitsdispositive lenken, deren Funktion darin liegt, "auf eine Realität zu antworten, so daß diese Antwort jene Realität aufhebt, auf die sie antwortet«. ${ }^{5}$ Im Verhältnis von Sicherheit und antimuslimischem Rassismus ist das Ziel einer gouvernementalen Führung, indirekt auf die Bevölkerung einzuwirken, also »auf die der Bevölkerung offensichtlich entfernten Dinge Einfluß [zu] nehmen ${ }^{6}{ }^{6}$

Bublitz zufolge handelt es sich bei der Gouvernementalität um »eine spezifische Form der Biomacht, in der das Leben des Menschen als Lebewesen auf dem Spiel steht und Gegenstand wissens- und technikbasierter >Praktiken des Selbst « wird «. ${ }^{7}$ Die Biopolitik, die Foucault bereits in Der Wille zum Wissen. Sexualität und Wahrheit I untersucht, nimmt seit dem 18. Jahrhundert die Bevölkerung - unter dem Vorwand innere und äußere Gefahren abwehren zu wollen - ins Visier biologischer Umgestaltung. Sie löst das alte Souveränitätsprinzip »sterben zu machen oder leben zu lassen « ab durch die Regel »leben zu machen oder in den Tod zu stoßen $\varkappa^{8}$ Die Biopolitik zielt auf die »vollständige Durchsetzung des Lebens «. ${ }^{9}$ Sie befragt Gesundheit, Sterblichkeit, Geburtenziffer, Hygiene und Lebensdauer der Bevölkerung. Ihre Geburtsstunde fällt nicht zufällig zusammen mit der

»Entwicklung eines spezifischen politischen Wissens und neuer Disziplinen wie Statistik, Demografie, Epidemiologie und Biologie, die Lebensprozesse auf dem Niveau von Bevölkerungen analysieren, um

\footnotetext{
5 Ebd.: 76.

6 Ebd.: 111.

7 Bublitz 2014: 94.

8 Foucault 2017b: 134.

9 Ebd.: 135.
} 
Individuen und Kollektive mittels korrigierender, exkludierender, normalisierender, disziplinierender, therapierender oder optimierender Maßnahmen zu >regieren « ${ }^{10}$

Als Beispiel besonders geeignet ist die sich im 18. Jahrhundert in Deutschland etablierende Polizeiwissenschaft, die, so Foucault, »eine vom Prinzip der Staatsräson beherrschte Regierungstechnologie angenommen « hat, indem sie sich den »Problemen der Population« annimmt, um die Bevölkerung »für die Stärke des Staates« $\mathrm{zu}$ >verbessern ${ }^{11}$ Das macht die Biopolitik zum Dreh- und Angelpunkt einer "Macht-Technologie, deren Gegenstand und Ziel das Leben ist $\aleph^{12}$, insofern sie (bestimmtes) Leben aufwertet, verlängert und vervielfacht. Sarasin ${ }^{13}$ hebt diesen Aspekt ausdrücklich hervor: Anders als es etwa in Agambens Souveränitätstheorie zum Tragen kommt, die sich unter dem Begriff der Biopolitik auf das Töten beschränkt, kreist Foucaults Konzept »um die Produktion, nicht um die Tötung von Leben «. ${ }^{14}$ Für Foucault stellt sich deshalb die Frage danach, wie eine Macht, die Leben maximieren und vervielfältigen will, töten kann: »Wie sollte eine Macht ihr höchstes Vorrecht in der Verhängung des Todes äußern, wenn ihre Hauptaufgabe darin besteht, das Leben $\mathrm{zu}$ sichern, $\mathrm{zu}$ verteidigen, $\mathrm{zu}$ stärken, zu mehren und zu ordnen? $^{15}$ Hier kommt der Rassismus ins Spiel.

Tatsächlich hängen Foucaults Überlegungen zur Biopolitik unmittelbar mit jenen zum modernen Rassismus zusammen: Der Rassismus legitimiert im Namen der Biopolitik das Töten. In seinem Vortrag In Verteidigung der Gesellschaft betont Foucault, dass die Biopolitik nicht ohne den Rassismus gedacht werden kann, Rassismus stellt eine intrinsische Komponente innerhalb des Machtapparates dar und ist das "Mittel, um in diesen Bereich des Lebens, den die Macht in Beschlag genommen

\footnotetext{
10 Lemke 2007: 14.

11 Foucault 2013a: 181.

12 Foucault 2001: 300.

13 Sarasin 2003: 58.

14 Ebd.: 61.

15 Foucault 2017b: 133.
} 
hat, eine Zäsur einzuführen: die Zäsur zwischen dem, was leben, und dem, was sterben muß «. ${ }^{16}$ Rassismus ermöglicht es, die Bevölkerung $\mathrm{zu}$ fragmentieren, $\mathrm{zu}$ disziplinieren und Gruppen gegeneinander auszuspielen, indem >Rassen ^produziert und als höherwertig bzw. minderwertig hierarchisiert werden. Wichtig dabei ist, dass Foucault unter dem Recht zu töten weit mehr versteht als den direkten Mord. Im Gegenteil zählen darunter auch indirekte Formen, wie »jemanden der Gefahr des Todes ausliefern, für bestimmte Leute das Todesrisiko oder ganz einfach den politischen Tod, die Vertreibung, Abschiebung usw. erhöhen ${ }^{17}{ }^{17}$

Zwar beschränkt sich Foucault in seinem Vortrag in erster Linie auf einen biologistischen Rassismus, doch kann, wie Rose anmerkt, ein ähnliches Prinzip ebenso im Falle eines kulturell argumentierenden Rassismus beobachtet werden. ${ }^{18}$ Mit Balibar und Hall ${ }^{19}$ versteht Rose Rassismus

»als machtvolles Operations- und Differenzierungsmodell [...], das in das Kontinuum einer Bevölkerung bedeutsame Unterscheidungen wie seigen oder >fremd durch diejenigen, für die die letztgenannten Kategorien in Anschlag gebracht werden, stärker als erstere einer Gefahr ausgesetzt sind «. ${ }^{20}$

Stoler kritisiert, dass in Foucaults enger Rassismus-Analyse, ausgehend von seiner Geschichte der Sexualität, der Kolonialismus zu kurz kommt. Schließlich spielen auch imperiale Schauplätze außerhalb Europas eine Rolle in der Produktion europäischer Diskurse um Sexualität, weshalb Foucault »die Praktiken [übergeht], welche eine Rassenterminologie in den Körper einschreiben ${ }^{21}{ }^{21}$ Bürgerliche Identitäten in europäischen Metropolen und in >ihren Kolonien in Übersee

\footnotetext{
16 Foucault 2001: 301.

17 Ebd.: 303.

18 Rose 2015: 334.

19 Balibar 1992; Hall 1994.

20 Rose 2015: 334.

21 Stoler 2002: 307.
} 
waren »insgeheim nachhaltig durch Rassenvorstellungen codiert «. ${ }^{22}$ Stoler hinterfragt, weshalb Foucault die Geburtsstunde des Rassismus ins späte 19. Jahrhundert legt, obgleich sich das Phänomen historisch, sozial und politisch breiter auffächern lässt. ${ }^{23}$ Auch Lemke vermisst die Berücksichtigung des Kolonialismus und die spezifischen Intersektionen zur Sexualität in Foucaults Kritik des Rassismus. Gleichzeitig geht er davon aus, dass dies analytische Vorteile bietet, indem Rassismus weder als "Ausnahmesituation oder Antwort auf soziale Krisen « ${ }^{24}$ noch als individuelle Handlung Einzelner verharmlost wird. Im Anschluss an Foucault kann Rassismus verstanden werden als Gesellschaft strukturierend, Politik leitend und institutionell manifestiert.

In Foucaults Überlegungen zur Gouvernementalität wird der Begriff der Biopolitik nicht mehr verwendet. In seiner weiteren Auseinandersetzung mit dem Problem der Bevölkerungsregierung spielt auch die Sexualität keine prominente Rolle mehr. Möglicherweise ist das der Grund, weshalb Foucault fortan dem Rassismus keine Beachtung mehr schenkt. Letztendlich hängt sie in seinen Überlegungen zur Biopolitik noch eng mit der sexuellen, und damit der biologischen Regulierung und (Um-)Gestaltung der Bevölkerung zusammen. Sarasin geht davon aus, dass Foucaults Konzept der Biopolitik dennoch »ein Torso mit vielen Schnittstellen für mögliche theoretische Weiterentwicklungen unter dem Stichwort >Gouvernementalität« « ist. ${ }^{25}$ Dies trifft gewiss für die Untersuchung des gegenwärtigen Rassismus unter dem Gesichtspunkt der Sicherheitsdispositive zu. Angesichts der Verquickung von (disziplinierender) Macht mit (normalisierenden) Selbsttechnologien stellt sich die Frage, wie sich rassistische Praktiken transformieren und wie das Subjekt zur Selbstführung angeleitet wird, wenn die >Freiheit und die >Sicherheit der Subjekte zur raison d'être liberaler Regierungspraxis werden. Die gouvernementale Macht setzt »in einer fundamentalen Weise nicht einfach die disziplinäre Anpassung und Unterwerfung

\footnotetext{
22 Ebd.: 308.

23 Ebd.: 312.

24 Lemke 2007: 59.

25 Sarasin 2005: 177.
} 
der Individuen voraus [...], sondern ganz im Gegenteil deren Freiheit «. ${ }^{26}$ Sicherheitstechnologien »fabrizieren eine Freiheit, die permanent bedroht und gefährdet ist und daher zur Grundlage immer neuer Interventionen wird, die einem Sicherheitskalkül der Gesellschaft folgen «. ${ }^{27}$ Doch was bedeuten Freiheit und Sicherheit im antimuslimischen Rassismus für die Führung rassifizierter Subjekte?

\section{Freiheit und (Selbst-)Führung im (antimuslimischen) Rassismus}

»Regieren heißt, die Dinge regieren ${ }^{28}$, schreibt Foucault. Das funktioniert über Erziehung, Kampagnen und Überzeugungsarbeit, um Einfluss auf die Gewohnheiten der Bevölkerung zu nehmen - auf ihre Befürchtungen, darauf, wie sie sich bewegen und verhalten, wie sie leben und denken. Auch mithilfe von Rassismus kann dieser Regel Folge geleistet werden. Rassismus legitimiert das Strafen, Ausschließen und Kriminalisieren, doch genauso gut können im Rassismus auch Einbezug, >Integration und Vereinnahmung funktional greifen, und das ohne die eigentliche Zielsetzung, nämlich rassifizierte Subjekte unterzuordnen, aus dem Blick zu verlieren.

Mecheril spricht von einem »antizipierten Rassismus « ${ }^{29}$, um $\mathrm{zu}$ beschreiben, dass Rassismus auch erfahren wird, indem man Geringschätzung und Degradierung vorwegnimmt oder Angriffe und Attacken befürchten muss. Rassifizierte Personen können deswegen Wut, Angst, Verzweiflung oder auch Scham empfinden. Mecheril beschreibt Vorsicht bis hin zur Vermeidung von Kontakten und dem Rückzug aus bestimmten Situationen als Reaktionen auf Rassismuserfahrungen.

Nach der Bedeutung des Sicherheitsdiskurses gefragt berichten unsere Gesprächspartner:innen von ihren Gefühlen angesichts der Alltäg-

$\begin{array}{ll}26 & \text { Ebd.: } 184 . \\ 27 & \text { Bublitz 2014: } 95 . \\ 28 & \text { Foucault 2017a: } 147 . \\ 29 & \text { Mecheril 1994: 60. }\end{array}$


lichkeit ihrer Rassismuserfahrungen. ${ }^{30}$ Ahmed diskutiert Rassismus im Zusammenhang mit einer "gewisse[n] paranoide[n] Sorge um Dinge, die bereits passieren und passieren könnten «, sodass sich rassifizierte Menschen als Effekt von Rassismuserfahrungen vorbeugend selbst zensieren und zurückziehen. ${ }^{31}$ Rassismus, Sicherheit und Gouvernementalität stehen so in einem wechselseitigen Dreiecksverhältnis: >Die Anderen können indirekt gelenkt werden, die Führung wird dezentralisiert und in die Subjekte selbst verlagert. ${ }^{32}$ Das lässt sich etwa am Interesse des Staates beobachten, einen spezifischen Bevölkerungsanteil als >Muslim:innen $\mathrm{zu}$ regieren, sie als solche $\mathrm{zu}$ adressieren und $\mathrm{zu}$ erziehen, ein Interesse, das einige muslimische Akteur:innen tatsächlich aufgreifen, unter anderem auch, weil es mit ihren Interessen zusammenfällt. Amir-Moazami spricht von einem »intime[n] Verhältnis von Anerkennungsgesten und verstärkter Kontrolle der religiösen Praxis durch Sicherheitstechniken «. ${ }^{33}$ Der hegemoniale Sicherheitsdiskurs trägt dazu bei, dass Muslim:innen zu >guten (deutschen)< Staatsbürger:innen zivilisiert und in die Logik liberal-säkularer Machttechniken eingefügt werden. Demzufolge betreten, so Amir-Moazami weiter, jene Muslim:innen, die sogenannte Sicherheitspartnerschaften eingehen, die öffentliche Bühne und arbeiten der staatlichen Islamismusbekämpfung zu. Staatlich gelenkte Imamausbildungen und die Gestaltung des islamischen Religionsunterrichts begreift sie als regulative Instrumente und Kontrollmechanismen mit dem Ziel, den Islam nach christlich-säkularem Vorbild zu zähmen und europagerecht zu modernisieren. Tezcan führt das am Beispiel der Deutschen Islamkonferenz

30 Zur Rekonstruktion der Emotionen: Keskinkılıç 2021 i.E.

31 Ahmed 2018: 125.

32 Auch weitere Subjektivierungen und Positionierungen bzw. ihre Intersektionen sind wirksam, da die hegemoniale Anrede als Muslim:in nur eine Facette des Lebens eines Menschen betrifft. Im Forschungsfeld Islam/Muslim:innen in Europa liegen Arbeiten vor, die die Zusammenhänge von Macht und Subjektivierung unter verschiedenen sozialen, historischen und politischen Gesichtspunkten thematisieren u.a. als Kritik am europäischen Säkularismus (AmirMoazami 2018; Asad 2003; Mahmood 2015). 
aus, die wesentlich zur Konstruktion »eines gesellschaftsfähigen, aufgeklärten, deutschen muslimischen Subjekts « ${ }^{34}$ beigetragen hat.

Die konzeptionelle Trennung von Herrschaft und Macht, die im neuen Begriff der Gouvernementalität notwendig wird, lenkt also den Blick auf jene rassistischen Verhältnisse, die sich abseits herkömmlicher Ausschlussmechanismen ausdrücken können. Im antimuslimischen Bedrohungsszenario werden Muslim:innen unter dem Aspekt der (De-)Radikalisierung und der Prävention angesprochen, ihrer (zugeschriebenen) Zugehörigkeit nach mit Terrorismus in Verbindung gebracht und dazu angehalten, >bösen Muslim:innen $\mathrm{zu}$ entsagen. ${ }^{35}$ Einerseits greift die Disziplinarmacht durch rechtliche Normen und disziplinierende Normierungen ${ }^{36}$ weiter ein, wie zum Beispiel in Form von Anti-Terror-Gesetzen, die überwachen, bestrafen und kriminalisieren. ${ }^{37}$ Andererseits wirkt bereits das Wissen, dass die Disziplinarmacht greifen kann, disziplinierend bzw. selbstführend.

Aus dem empirischen Material der vorliegenden Studie geht hervor, dass unsere Gesprächspartner:innen ihr Verhalten nach dem Wissen, dass potentiell alle Muslim:innen betroffen sind (»wir sind auch Gefährder«), ausrichten und sich selbst kritisch befragen, ob sie als Gefahr wahrgenommen werden könnten. Sie antizipieren Rassismuserfahrungen und loten Handlungsmöglichkeiten aus, die sie nicht allzu sehr in Gefahr bringen.

Hieran setzt unser Forschungsinteresse an: Wir untersuchen die Aufforderung bzw. Anordnung, sich selbst zu führen und stellen die Frage, ob und gegebenenfalls wie das geschieht. Uns interessiert, wie es möglich ist, auf diese Aufforderung zu antworten (ohne sich gänzlich $\mathrm{zu}$ unterwerfen) und dabei eigene Interessen nicht aus dem Blick $\mathrm{zu}$ verlieren oder sogar ins Spiel $\mathrm{zu}$ bringen - also widerständig $\mathrm{zu}$ handeln. Uns interessieren in diesem Sinne, wie Gemeinden, Verbände und Vereine auf Ein- und Ausschlussmechanismen im Zusammenhang 
mit dem Sicherheitsdispositiv reagieren. Oder um es in gouvernementalitätstheoretischer Perspektive zu formulieren: Wie macht sich das muslimische Subjekt die gouvernementale Vernunft angesichts des antimuslimischen Rassismus zunutze, um Freiheiten zu erweitern und Handlungsspielräume (ob unterwerfend oder widerständig) im Spannungsverhältnis von Macht- und Selbsttechniken zu dehnen und eigene Bedürfnisse zu artikulieren? Denn wie bereits Foucault zu bedenken gibt, folgt die gouvernementale Vernunft keinesfalls starr den Staatsinteressen, sondern erwächst aus dem Zusammenspiel individueller und kollektiver Bedürfnisse und Interessen. Es gibt keine universelle Freiheit, eher »ein Verhältnis, bei dem das Maß des >zu wenig ‘ an bestehender Freiheit durch das >noch mehr $<$ an geforderter bestimmt wird «. ${ }^{38}$

\section{Subjekt, Widerstand und Handlungsmacht}

Wo Macht ausgeübt wird, gibt es auch Konflikte und Strategien, sich ihr zu widersetzen, sich aufzulehnen oder alternative Handlungsoptionen auszuloten. Um zu verstehen, wie Macht operiert, "müssen wir vielleicht die Widerstände dagegen untersuchen und die Bemühungen, diese Beziehungen aufzulösen «, schreibt Foucault. ${ }^{39}$ Mehr noch: Es gibt »keine Machtbeziehung ohne Widerstand, ohne Ausweg oder Flucht, ohne möglichen Umschwung ${ }^{40}{ }^{40}$ Das eine existiert nicht ohne das andere: Macht und Widerstand stehen »in einem Verhältnis wechselseitiger Provokation, endloser Verkettung und ständiger Verkehrung «. ${ }^{41}$

Foucault lädt dazu ein, den Gegenstand unter dem Aspekt des Widerstandes, also der Umgangsweisen und Handlungsoptionen $\mathrm{zu}$ beleuchten. Schließlich fokussiert er in seinen Studien zur Gouvernementalität keine totalitären Gesellschaften. Flügel-Martinsen vermutet,

\footnotetext{
38 Foucault 2017b: 97.

39 Foucault 2013b: 243.

40 Ebd.: 261.

41 Ebd.: 262.
} 
dass »der Prozess der politischen Subjektwerdung erst durch die interne Verschränkung von Unterwerfung und Widerstand verständlich ${ }^{42}$ wird. Beide Aspekte schließen sich weniger gegenseitig aus, als dass sie sich vielmehr wechselseitig bedingen. Der entscheidende Punkt für die Frage des Widerstands liegt darin, dass im schöpferischen Prozess der Macht Subjekte nicht einfach unterworfen, sondern hervorgebracht werden. Im Zusammenspiel von Subjektwerdung und Macht liegt ein Widerstandspotential: Die Subjekte »selbst sind es ja, die im Konflikt der Mächte mitwirken und die so, wie es erst in diachroner Perspektive sichtbar wird, die Diskurse der Macht umschreiben«. ${ }^{43}$

Den Aspekt subversiver Widerstandspraktiken im Kontext hegemonialer Anrufungen greift Butler in Haß spricht auf. Sie bekräftigt, dass Subjekte zwar diskursiv hervorgebracht werden, diese aber zeitgleich durch den Namen, mit dem sie angerufen werden, in eine Sphäre treten, in der sie den eigenen Handlungsraum entgegen des ursprünglichen Interesses der Anrede erweitern können:

»Durch den Namen, den man erhält, wird man nicht einfach nur festgelegt. Insofern dieser Name verletzend ist, wird man zugleich herabgesetzt und erniedrigt. Doch enthält der Name auch eine andere Möglichkeit, da man durch die Benennung auch eine bestimmte Möglichkeit der gesellschaftlichen Existenz erhält und erst in ein zeitliches Leben der Sprache eingeführt wird, das die ursprünglichen Absichten, die der Namensgebung zugrunde lagen, übersteigt. Während also die verletzende Anrede ihren Adressaten scheinbar nur festschreibt und lähmt, kann sie ebenso eine unerwartete, ermächtigende Antwort hervorrufen. ${ }^{44}$

42 Flügel-Martinsen 2014: 44.

43 Ebd.: 54.

44 Butler 2006: 10. 
Butler knüpft in ihren Arbeiten im Anschluss an Austin ${ }^{45}$ an Sprechakttheorien und den Performativitätsbegriff an. ${ }^{46}$ Theorien des Performativen fokussieren nicht ausschließlich das Feld der Linguistik, sondern werden »über die Sprache hinaus auf sinnenhafte Kulturphänomene aller Art übertragen «. ${ }^{47}$ Wie König bemerkt, unterscheidet sich Butlers Performativitätsbegriff grundlegend von einem theaterwissenschaftlichen Verständnis, in welchem das »)Ereignishafteく (der Aufführung) als fundamental« angesehen wird, während Butler das Prozesshafte hervorhebt. ${ }^{48}$ Butler bietet eine Perspektive an, »wie sprachliche bzw. diskursive Praxis wirklichkeitsverändernd [H.i.O.] wirken kann - nämlich

45 In How to do things with Words begreift Austin das Sprechen, etwa Beleidigungen, Befehle aber auch Ansprache und Rede, als eine Form des Handelns. Das bedeutet, die sprachliche Handlung konstituiert das, was sie äußert. Austin (1975: 109ff.) unterscheidet zwischen drei verschiedenen Sprechakten: Der lokutionäre Akt meint die Handlung, etwas zu sagen. Dazu zählen sprachliche Laute und Äußerungen nach grammatikalischen Regeln einer Sprache, die dazu dienen, Aussagen über Cegenstände und Ereignisse zu machen. Unter einem illokutionären Akt, »meaning in the traditional sense«, versteht Austin den Vollzug einer konventionellen Sprachhandlung, die sich aus einer sozialen Interaktion ergibt, also Fragen, Drohungen oder Empfehlungen. Der illokutionäre Akt vollzieht sich, indem etwas geäußert wird. Dagegen betrifft ein perlokutionärer Akt (»what we bring about or achieve by saying something«) die Folgen einer Sprachhandlung, also dadurch, dass etwas geäußert wird. Er betrifft die Wirkung, er kränkt, verunsichert oder überzeugt.

46 In Anlehnung an Austins Sprechakttheorie und unter Berücksichtigung der Foucaultschen Diskurstheorie erweitert Butler das Blickfeld um Phänomene der Identitätskonstruktionen, insbesondere im Zusammenhang mit der Performativität von Geschlecht und Sexualität: »Cender identity is performative, which means [...] that it is real only to the extent that it is performed. [...] If gender attributes, however, are not expressive but performative, then these attributes effectively constitute the identity they are said to express and reveal.« (Butler 1990: 278f.) Indem Butler auf die Rolle des Performativen in der Herausbildung von Ceschlecht verweist, weist sie Vorstellungen einer ontologischen Realität von Ceschlechtsidentität, also einer vermeintlich natürlich gegebenen, zurück.

47 Hempfer/Volbers 2011: 7.

48 König 2001: 45. 
durch die Möglichkeit der Variation oder parodistischen Subversion vorgegebener Handlung - oder Identitätsskripte «. ${ }^{49}$

Der Mechanismus lässt sich auch im antimuslimischen Rassismus beobachten. Hegemoniale Sprechhandlungen, die Muslim:innen im Akt des Bezeichnens und Besprechens als Sicherheitsbedrohung hervorbringen, können im Sinne Austins neben dem illokutionären Akt auch als perlokutionärer Akt verstanden werden. Die Diskriminierung vollzieht sich einerseits, indem antimuslimisch gesprochen wird, aber auch weil Ausschluss und Benachteiligung praktische Folgen davon sind, dass antimuslimisch gesprochen wird. Die Bezeichnung ist keine neutrale, im Gegenteil reguliert sie den Zugang zu Ressourcen, entscheidet darüber, wer (zu welchen Themen und wie) Gehör findet.

Mit dem Akt der Benennung werden Muslim:innen also dem Diskurs unterworfen und als >Muslim:innen $\prec$ hervorgebracht. Butler nennt diesen Prozess Subjektivation. Die Unterwerfung des Subjekts unter den Diskurs führt es zugleich ins Leben und ist eine Voraussetzung dafür, zu sprechen bzw. Gehör zu finden. Die Anrufung des Subjekts setzt die Bedingungen, um sich selbst im Angesicht der Macht zu konstituieren und sich Diskursregeln zu eigenen Zwecken nutzbar zu machen. Butler schreibt, »dass gerade darin, dass der herrschende, autorisierte Diskurs enteignet [H.i.O.] werden kann, eine Möglichkeit seiner subversiven Resignifikation liegt ${ }^{50}$

Das Changieren zwischen Unterwerfung und Handlungsmacht ist durchaus ambivalent zu begreifen, wie Gutiérrez Rodríguez am Beispiel (neo-)liberaler Regierungstechniken in der Ausländer-, Migrations- und Asylpolitik zeigt. Jene, die als >Migrant:innen und als >Ausländer:innen angerufen werden, werden $\mathrm{zu}$ >ethnisierten Anderen konstruiert: "Erst von dem Zeitpunkt an, an dem die Minderheiten als die rethnisch Differenten kodifiziert, kontrolliert und regiert werden, existieren sie als solche. $\aleph^{51}$ Sie werden zu defizitären Anderen konstruiert,

49 Butler 2011: 49.

50 Ebd.: 246.

51 Gutiérrez Rodríguez 2003: 171. 
in ihren Bewegungsmöglichkeiten eingeschränkt und für ihre missliche Lage selbst verantwortlich gemacht. Gleichzeitig werden sie aufgefordert, sich zu integrieren und durch Leistung und Arbeit aufzusteigen. Indem der Staat an die Individuen appelliert, entledigt er sich seiner Verantwortung. Dem Topos des homo oeconomicus unterworfen, werden Migrant:innen paradoxerweise einerseits entmächtigt und andererseits als selbstständig handelnde Subjekte adressiert. Die Anrufung als ethnisierte Andere geht in das Selbstverständnis der Individuen ein, ohne sie total zu bestimmen. Gutiérrez Rodríguez weist darauf hin, dass sich die Anrufung verkompliziert: Die Anderen gelten als Sündenbock und Problem und werden gleichzeitig »als die neuen UnternehmerInnen [H.i.O.] zelebriert ${ }{ }^{52}$ und als ökonomische Zielgruppe zur Förderung von Selbstständigkeit angesprochen. Eine direkte Analogie hierzu findet sich in der steigenden Anzahl an Förderprogrammen zur Deradikalisierung von Muslim:innen, die sich insbesondere an muslimische Selbstorganisationen richten und finanzielle Abhängigkeiten schaffen. ${ }^{53}$ Die Biopolitik bindet >die Anderen in die neoliberale Logik ein, vermag sie aber nicht vollständig zu kontrollieren:

»Denn der Versuch der Domestizierung der Subjekte scheitert an der Hartnäckigkeit der unbestimmbaren und ungezügelten kollektiven Praxis. Die staatlichen Führungsprogrammatiken im Sinne der Couvernementalität mögen daher aus den Praktiken und Rationalitäten der Subjekte schöpfen, können diese aber nicht vollständig abbilden und kontrollieren. Die Praxis der Subjekte ist nicht zu fixieren, sie durchdringt die Risse der Strukturen, in denen sie zwar verfangen, aber auch zugleich beweglich ist. Das Konzept der Gouvernementalität wirft daher spannende Fragen bezüglich der Vereinnahmung subjektiver Praktiken und Denkformen durch staatliche Institutionen auf. Zugleich verweist es auf die Grenzen dieser Führungslogik, die an

\footnotetext{
52 Ebd.: 176.

53 Attia 2019b; Qasem 2019.
} 
der Praxis und dem widerspenstigen Begehren der Subjekte, die sie zu modellieren sucht, scheitert. ${ }^{54}$

Ähnlich argumentiert auch Rose. Mit Foucault und Butler definiert sie widerständiges Handeln als »eine Praxis der Grenzbearbeitung, eine Praxis des kritischen Fragens und einer kritischen Haltung, die genau jene bislang gültigen Grenzziehungen zwischen dem >Normalen dem >Verwerflichen zum Gegenstand der Auseinandersetzung macht, indem sie ihnen nur begrenzt entspricht ". ${ }^{55}$ Auch Wagenknecht befragt die widersprüchlichen und ambivalenten Verhaltensweisen im Umgang mit hegemonialer Anrede und lenkt dazu den Blick auf die in der Lebenspraxis von Subjekten gesammelten Erfahrungen und Bedürfnisse, »die Ausgangspunkt für die Überwindung herrschaftlicher Zurichtung sein können. $\ll^{56}$ Die Möglichkeit, den Namen zurückzuweisen und das Selbst abseits der diskursiven Anrufung zu gestalten, gelingt, indem Ambivalenz produziert wird. Die Anrufung eines Subjekts kann auf eine Weise beantwortet werden, die die Ansprache irritiert. Sie vermag es, die hervorgebrachte Repräsentation, unter der das Subjekt benannt wird, zu stören und zu brechen.

»Die verschiedenen ökonomischen, politischen, ideologischen, kulturellen usw. Prozesse der Ambivalenzproduktionen gehören zu einer wesentlichen Veränderung des gesellschaftlichen Ganzen, zur Formierung einer neuen Produktionsweise. [...] Die Ambivalenz entsteht als Veränderung in der Artikulation gesellschaftlicher Widersprüche, in der Art und Weise, wie sie verbunden sind und sich gegenseitig formen. Vormals klare Differenzlinien werden verwischt, werden nicht nur durch ideologische Rede vernebelt, sondern tatsächlich uneindeutig. ${ }^{57}$

\footnotetext{
54 Gutiérrez Rodríguez 2003: $176 f$.

55 Rose 2015: 332.

56 Wagenknecht 2003: 200.

57 Ebd.: 213.
} 
Flügel-Martinsen bringt es folgendermaßen auf den Punkt: »Widerstand bedeutet so gesehen Bedeutungsverschiebung «. ${ }^{58}$

Aus der Skepsis gegenüber metaphysisch überhöhten Begrifflichkeiten und absoluten Wahrheiten, die Foucaults Arbeiten durchziehen, warnt Flügel-Martinsen davor, eine systematische Theorie des Widerstandes bei Foucault zu suchen, und plädiert stattdessen dafür, die Frage nach widerständigem Handeln umzudeuten:

» $Z u$ fragen ist also nicht, wie Subjekte generell Widerstand gegen Machtprozesse, die sie konstituieren, leisten können, sondern zu untersuchen sind die je spezifischen Modalitäten der Subjektkonstitution und des Widerstands in konkreten historischen Konstellationen ${ }^{59}$

Jain geht davon aus, dass die Ambivalenz und Widersprüchlichkeit von Wünschen und Begierden eine Reflexion ermöglichen, die wiederum Widerspruch und infolge dessen auch »die Spannung zwischen Subjekt und Politik, die die eigentliche Grundlage des politischen Verhältnisses ist, wieder beleben $«{ }^{60}$ Er sieht in der Entwicklung eines solchen Subjektbewusstseins die notwendige Voraussetzung für kollektive politische Handlungsfähigkeit. Das Subjekt muss

»[...] sich selbst als solches erkennen und begreifen und sich zugleich (kritisch und widerständig) auf seine Umwelt beziehen. Es muss ein sauthentisches< Bewusstsein seiner Selbst entwickeln, und dies setzt eine (Selbst-) Reflexion voraus, die gerade für die Widersprüche und die Ambivalenz des Seins und des Selbst offen ist. Ein solcher reflexiver Authentizitätsbegriff bedeutet, dass das Selbst seine immanente, nicht transzendierbare innere Widersprüchlichkeit und Ambivalenz entdeckt, um seinen Widerspruch für die Möglichkeit des Widersprüchlichen und des Differenten zu entfachen. Die reflexive Authentizität des Selbst bestünde also gerade in der Spiegelung und im Zu-

\footnotetext{
58 Flügel-Martinsen 2014: 54.

59 Ebd.: 56.

60 Jain 2003: 255.
} 
lassen von innerer wie äußerer Ambivalenz - nicht im Beharren auf Eindeutigkeit und Identität. « ${ }^{61}$

Das Ambivalenz-Dispositiv, das in diesen Überlegungen als widerständige Reaktion auf eine unterwerfende Anrede bemüht wird, lenkt den Blick auf vielfältige Strategien, mit denen auch Muslim:innen auf den antimuslimischen Sicherheitsdiskurs antworten. Butlers Subjektivationstheorie gemäß werden sie als (Gefahren-)Subjekt angerufen und hervorgebracht, ihnen wird ein Name und damit einhergehend eine gesellschaftliche Rolle zugewiesen, die sie ins Leben führt und zugleich die Bedingungen schafft, um handlungsfähig und gehört zu werden. Themen, die an sie herangetragen werden - Salafismus, Terrorismus, (De-)Radikalisierung und Prävention - können als Muslim:innen Angerufene aufgreifen, sich $\mathrm{zu}$ ihnen positionieren und sich mit ihnen auseinandersetzen und zugleich eigene Erfahrungen, Interessen und Bedürfnisse artikulieren.

Das erlaubt, islambezogene Kontrastfolien $\mathrm{zu}$ irritieren und um andere Identifikationspunkte $\mathrm{zu}$ erweitern, unter denen sich die jeweiligen Lebensentwürfe und -realitäten gestalten, Erfahrungen mit dem antimuslimischen Rassismus zu artikulieren und das muslimische Selbst $\mathrm{zu}$ verkomplizieren, statt Identität $\mathrm{zu}$ vereinheitlichen. Essentialistische Anrufungen als >Muslim:in können zurückgewiesen (und damit verweigert) werden, sie können unter Berücksichtigung verschiedener religiöser, säkularer, politischer, sozialer und vergeschlechtlichter Deutungsmuster differenziert und multipliziert werden, sodass (nicht nur individuell) Ambivalenz produziert wird. Die »Praxen dieser Überwindung [sind] notwendig kollektive Praxen ${ }^{62}$, gerade weil Erfahrungen des antimuslimischen Rassismus strukturell eingeschrieben sind und im größeren sozialen Zusammenhang existieren. (Als) Muslim:innen (Markierte) sind sich der kollektiven Erfahrungsdimension bewusst. Sie verschaffen sich gegenseitig Gehör,

61 Ebd.: $242 f$.

62 Wagenknecht 2003: 200. 
beziehen sich aufeinander und denken gemeinsam konkrete Utopien. Die Erfahrung von `Gleichheit kann mit einer »Solidarität der Verschiedenheiten ${ }^{63}$ verknüpft werden.

\section{Schlussfolgerung}

In der Auseinandersetzung mit dem antimuslimischen Rassismus kommt man nicht umhin, dem zentralen Stellenwert des hegemonialen Sicherheitsdiskurses Rechnung zu tragen. Wie aus dem empirischen Material der vorliegenden Studie hervorgeht, sind unsere muslimischen Gesprächspartner:innen in ihrem Alltag und in ihrem Beruf, in öffentlichen Institutionen und auf offener Straße, durch mediale und politische Ansprache mit Misstrauen und Verdacht konfrontiert. Sie müssen sich verantworten und Stellung beziehen, sich zu >islami(sti)scher Gewalt positionieren, sich distanzieren und als 'guter Ausnahmen in Szene setzen. Die Erfahrung, ihrer (vermeintlichen) Religion, Kultur und Herkunft nach kollektiv als Gefahr wahrgenommen zu werden, hat Einfluss auf ihre Gefühle und ihr Selbstbild, darauf, an welchen Orten sie Präsenz zeigen und welche sie wiederum meiden, ob und wie sie Religiosität praktizieren, wie sie sich kleiden, was und wie sie (be-)sprechen oder (ver-)schweigen. Doch Muslim:innen sind wie andere auch - keine passiven Objekte einer allgegenwärtigen und omnipotenten Macht.

Hieran knüpft unsere Studie an: Wir fragen danach, wie das muslimische Subjekt Deutungs- und Handlungsmacht (zurück-)erlangt. Vor dem Hintergrund von Foucaults Ausführungen zum Verhältnis von Sicherheit, Bevölkerung und Gouvernementalität sowie seinem Diktum, dass es dort, wo es Macht gibt, auch Widerstand gibt, fragen wir danach, wie die als Muslim:innen Angerufenen das Verhältnis von Unterwerfung und Widerstand angesichts des Sicherheitsdiskurses austarieren. Uns stellt sich also die Frage nach den Spielräumen: Wie navigieren Muslim:innen durch Strukturen der Verbesonderung 
und Diskriminierung, durch Ein- und Ausschlüsse, durch Stigmatisierung und Generalverdacht? Wie knüpfen sie an gesellschaftliche Debatten um Sicherheit an, wie beteiligen sie sich an Aushandlungsprozessen und wie positionieren sie sich, um sich Gehör für ihre Erfahrungen, Anliegen und Bedürfnisse zu verschaffen? Wie gelingt es >Muslim:innen am Diskurs teilzuhaben und dabei Definitions- und Handlungsmacht (wieder) zu gewinnen? Dabei interessieren uns auch widersprüchliche und uneindeutige Gleichzeitigkeiten von Unterwerfung und Widerstand, etwa wie in der affirmativen Selbstführung auch seigene ‘ Interessen artikuliert und verfolgt werden können. Ausgehend davon, dass im Machtverhältnis Aushandlungspotential liegt und in der Selbstführung Interpretations- und Handlungsspielräume genutzt werden können, ist es also möglich, dass sich Muslim:innen im Prozess und in der Aneignung der sicherheitspolitischen Selbstführung nicht nur anpassend unterwerfen, sondern im Zuge ihrer Antwort den Sicherheitsdiskurs verschieben und ihn nutzen, um anderen Interessen nachzugehen als jenen, die von ihnen erwartet werden.

Schließlich lässt sich im empirischen Material ein weites Archiv an widerständigen Selbstbildern, Verhaltensweisen und alternativen Handlungsoptionen nachvollziehen, die das Gegenstück zur antimuslimischen Regierungskunst der Gouvernementalität bilden. Um es in Foucaults Worten $\mathrm{zu}$ sagen, geht es um »die Kunst nicht regiert zu werden bzw. die Kunst nicht auf diese Weise und um diesen Preis regiert zu werden «. ${ }^{64}$ Unsere Gesprächspartner:innen sind darum bemüht, zwischen verschiedenen gesellschaftlichen Rollen $\mathrm{zu}$ changieren und sie miteinander in Dialog zu bringen. Sie berichten von Rassismuserfahrungen, setzen sie in einen politischen, historischen Zusammenhang und untermauern zugleich professionelle, wissenschaftliche und aktivistische Sprechpositionen. Sie ordnen und verkomplizieren gesellschaftliche Themen, die an sie herangetragen werden, sie weiten Fragestellungen aus, analysieren und kontextualisieren eigene Positionierungen, differenzieren Diskussionsräume aus und untergraben hegemoniale Sprechakte.

64 Foucault 1992: 12. 
Insgesamt fordern sie defizitäre Islamdebatten heraus, irritieren homogene Kategorien und rücken komplexe Lebensrealitäten ins Feld, indem sie auf globalhistorische, queer-feministische, unterschiedlich religiöse, säkulare und weitere Erfahrungen und Erinnerungen zurückgreifen. Sie sind sich der Ausschlussmechanismen des Sicherheitsdiskurses bewusst, reflektieren Anforderungen nach Prävention und Deradikalisierung unter dem Aspekt des antimuslimischen Rassismus und rücken eigene Sicherheitsbedürfnisse und staatsbürgerliche Interessen ins Zentrum, die sie den Medien, der Politik und der Gesamtgesellschaft gegenüber geltend machen. Sie kritisieren ein Klima des Misstrauens, der Verdächtigungen und Konkurrenzen, das Einfluss auf die Community und die Zusammenarbeit untereinander nimmt, und reflektieren angesichts dessen Möglichkeiten der Solidarisierung. Und sie tauschen Visionen für eine bessere, community-nahe Zusammenarbeit aus, in der sie nicht gegeneinander ausgespielt werden, sondern stattdessen Räume zur Selbstermächtigung gestalten. 


\section{Rekonstruktive Forschungsmethoden rassismuskritisch perspektiviert}

Im Bedrohungsszenario des rislamistischen Terrorismus werden Muslim:innen als Subjekte hervorgebracht. Sie haben sich zum Sicherheitsdiskurs zu positionieren und ergreifen hierzu diverse Handlungsoptionen, um auf Anforderungen und Anrufungen zu reagieren, (nicht oder anders) $\mathrm{zu}$ antworten und eigene Themen und Bedürfnisse einzubringen. Mit Foucault gehen wir davon aus, dass das gesellschaftliche Kräfteverhältnis keines zwischen aktiv Herrschenden und passiv Beherrschten ist: Macht wird nicht von einer Gruppe besessen, während andere gänzlich ohnmächtig sind. Vielmehr wirkt Macht in sozialen Beziehungen und stellt »eine bestimme Form augenblickhafter und beständig wiederholter Zusammenstöße « ${ }^{1}$ dar. Mit der vorliegenden empirischen Studie versuchen wir, jenen komplexen Prozessen Rechnung zu tragen, in denen Muslim:innen als soziale Größe produziert werden und in denen sie sich zugleich selbst hervorbringen. Es bieten sich rekonstruktive Forschungsmethoden und insbesondere die Dokumentarische Methode an, um Verhandlungen zwischen $>$ Muslim:innen $<u$ untersuchen, während die Objektive Hermeneutik sich für das Herausarbeiten der Positionierungen einzelner `Muslim:innen als nützlich erweist. Beide Methoden unterscheiden sich in ihrem Erkenntnisinteresse und -ziel: Während mithilfe der Dokumentarischen Methode der Umgang mit der bestehenden, gesellschaftlichen Wirklichkeit zu erklären versucht wird, 
zielt die Objektive Hermeneutik darauf, die Sinnstrukturierung dieser Wirklichkeit zu rekonstruieren und zu verstehen.

Im Rahmen unserer Auseinandersetzungen mit beiden Methoden wurden wir auf verschiedene Probleme aufmerksam, die mit erkenntnistheoretischen und forschungsethischen Fragestellungen zusammenhängen. Dies zog Konsequenzen für den Erhebungs- und Auswertungsprozess unserer Daten nach sich: Wir konnten in den Methodologien beider Methoden keine systematische Berücksichtigung gesellschafts- oder gar rassismuskritischer Ansätze erkennen, die es ermöglicht hätten, die Komplexität und Widersprüchlichkeit der Erfahrungen und die Gleichzeitigkeit verschiedener, auch widerstreitender sozialer Wirklichkeiten von rassifizierten Menschen deutend $\mathrm{zu}$ verstehen. Das geteilte, reflektierte Erfahrungswissen rassifizierter Personen wird im sogenannten »Regelwissen ${ }^{2}$ der Objektiven Hermeneutik oder im »kommunikative[n] Wissen $\aleph^{3}$ der Dokumentarischen Methode nicht als common sense der Gesamtgesellschaft abgebildet. Rassifizierte Personen greifen aber sowohl auf ein gesamtgesellschaftliches Regel- bzw. kommunikatives Wissen als auch auf ein Communitybasiertes zurück, das ergab unsere Analyse. Um also einen reflektierten Zugang zu Erfahrungen, Orientierungen und Handlungspraxen $\mathrm{zu}$ ermöglichen bzw. nicht durch eigene, unbearbeitete Verstrickungen $\mathrm{zu}$ behindern, sollte der Forschungspraxis zur Interpretation und Analyse von Datenmaterial mit rassifizierten Menschen eine macht- und rassismuskritische Auseinandersetzung vorangehen. Die Entwicklung der Fragestellung und des Forschungsdesigns, der Zugang zum Feld, die Erarbeitung von Diskussionsimpulsen und Leitfragen, die Vorbereitung auf die Datenerhebung und der Prozess der Interpretation und Generalisierung müssen rassismuskritisch reflektiert werden, um der rassistischen Strukturierung der gesellschaftlichen Wirklichkeit Rechnung tragen zu können.

Bohnsack, Przyborski und Schäffer argumentieren, dass die Analyse mithilfe der Dokumentarischen Methode stets im Zusammenhang mit

2 Wernet 2009: 14.

3 Bohnsack 2018: 54. 
»unserer eigenen Praxis des Forschens $\aleph^{4}$ steht. Wernet beschreibt für die Objektive Hermeneutik, dass Interpret:innen mit den Regeln sozialer Handlungen vertraut sind, da sie als »sprachlich-handlungsfähige Subjekte« gelten, sodass sie »keine Vertrautheit mit dem Forschungsgegenstand $\aleph^{5}$ aufweisen müssen. In Abgrenzung dazu stellen wir im Folgenden unsere Forschungspraxis und modifizierte Anwendung der genannten rekonstruktiven Forschungsmethoden mit (als) Muslim:innen (Markierten) vor dem Hintergrund des antimuslimischen Rassismus vor.

\section{Sample, Interviewführung und das Problem mit den Typologien}

Bezüglich der Dokumentarischen Methode orientierten wir uns an den Vorlagen für Gruppendiskussionsverfahren ${ }^{6}$ und luden (als) Muslim:innen (Markierte) zu drei Gruppendiskussionen ein, um vor dem Hintergrund ihrer beruflichen, ehrenamtlichen, aktivistischen, künstlerischen oder wissenschaftlichen Tätigkeit und ihrer Erfahrungen mit dem antimuslimischen Rassismus zu sprechen. Wir baten sie, miteinander darüber ins Gespräch zu kommen, wie sie den Sicherheitsdiskurs wahrnehmen, welche Bedeutung er für sie hat und wie sie damit umgehen. Entsprechend der Methodologie der Objektiven Hermeneutik führten wir anschließend zehn "gesprächsförmige « ${ }^{7}$ Einzelinterviews mit (als) Muslim:innen (Markierten) zur gleichen Fragestellung. Vor der Datenerhebung entwickelten wir ein Datenschutzkonzept für den gesamten Forschungsprozess. Zur Erhebung bereiteten wir offene Diskussionsimpulse bzw. Leitfragen zur Orientierung vor. Für die Interpretationen wählten wir jeweils drei Sequenzen aus, transkribierten und interpretierten sie mit dem Ziel, den Orientierungsrahmen unserer Diskussionsteilnehmer:innen und die Handlungspraxis unserer

4 Bohnsack/Przyborski/Schäffer 2010: 13.

5 Wernet 2009: 14.

6 Bohnsack/Przyborski/Schäffer 2010.

7 Wernet 2009: 58. 
Gesprächspartner:innen sowie die Strukturen, innerhalb derer sie sich bewegen, zu rekonstruieren.

Die Gruppenzusammensetzung erfolgte nach thematischen Gesichtspunkten, wobei wir uns bemühten, sie entlang unterschiedlicher Aspekte intersektional zu pluralisieren. Das weicht von der üblichen Vorgehensweise $a b$, Gruppen als shomogene, künstliche oder sheterogene, natürliche oder einer Kombination aus beiden Elementen ${ }^{8}$ zusammen zu setzen und danach $\mathrm{zu}$ ordnen, wo »sich in ihnen ein Kontrast in den Orientierungsrahmen, aber auch in Bezug auf die Erlebnisschichtung finden " 9 lässt. Grund hierfür ist, dass es nicht unser Anliegen ist, eine vergleichende Erkenntnishaltung einzunehmen und die Besonderheit eines Falles vor dem Hintergrund anderer Fälle $\mathrm{zu}$ vergleichen, sondern möglichst differenziert Deutungen und Strategien im Umgang mit ähnlichen Erfahrungen zu rekonstruieren. Machtkritische und intersektionale Theorien $\mathrm{zu}$ Rassismus (und $\mathrm{zu}$ anderen gesellschaftlichen Machtverhältnissen) weisen überzeugend darauf hin, dass die Festlegung von Personen auf Kategorien und die Suche nach stabilen Identitäten den fluiden, widersprüchlichen und komplexen Verstrickungen in gesellschaftliche Machtverhältnisse zuwiderläuft. Obwohl rassistische Topoi und ihre Effekte klar beschrieben werden können, wirken sie zusammen mit anderen Machtverhältnissen und werden von Subjekten in jeweils konkreten Situationen ausgehandelt. Insofern würde die Identifizierung von Fällen hinter den Forschungsstand $\mathrm{zu}$ (antimuslimischem und intersektionalem) Rassismus und zur diskursiven Hervorbringung des >muslimischen Subjekts` zurückfallen - und nicht substantiell zur Beantwortung unserer Forschungsfrage beitragen. Wir nehmen daher die Interrelation von Religion, Kultur, Herkunft, Klasse, Alter, Ability, Geschlecht und Sexualität in den Blick, ohne sie als Personenmerkmale $\mathrm{zu}$ verstehen. Vielmehr verstehen wir sie als vielfach verwobene und sich wechselseitig bedingende gesellschaftliche Verhältnisse, die jeweils konkrete Effekte in konkreten Situationen haben und auf die

8 Lamnek/Krell 2016: 410.

9 Amling 2015: 99. 
in changierenden und widersprüchlichen Weisen Bezug genommen wird. Aus diesem Grund erheben wir auch keine Sozialdaten, sondern arbeiten die Bezugnahmen der Gesprächspartner:innen auf Sozialdaten, Personenmerkmale und gesellschaftliche Machtverhältnisse in ihrer Bedeutung für den jeweiligen Kontext heraus.

Anstatt also essentialistische Größen als Ausgangspunkt zu setzen und soziale Machtverhältnisse in Personenkategorien zu überführen und zu festigen, gehen wir von Dynamiken aus und richten unsere Aufmerksamkeit auf jene sozialen Verhältnisse, in denen sich Macht manifestiert. Wir stellen die realen Folgen der gesellschaftlichen Konstruktion von Kategorien in Rechnung, indem wir sie in der Rekonstruktion konkreter Sequenzen als existierende Verhältnisse berücksichtigen, arbeiten jedoch im kollektiven Forschungsprozess daran, sie nicht zu reproduzieren. Wir fragen nach den Ordnungen und Strukturen als Rahmenbedingungen, die es ermöglichen, dass der Diskurs und die Dinge, die er ordnet, sich gerade auf diese Weise (und keine andere) gestalten.

Argumentationen und Strategien zum Umgang mit der Adressierung von >Muslim:innen als Sicherheitsrisiko variieren, sie changieren selbst in den Argumentationen ein und derselben Person im Laufe des Interviews oder der Diskussion. Rekonstruktionen, die darauf zielen, sozio- und sinngenetische Typen zu generieren ${ }^{10}$, würden dies als widersprüchlich und inkonsistent deuten und zum Anlass nehmen, neue Typen zu bilden. Unser Erkenntnisinteresse bezieht sich aber nicht darauf, Typen von Muslim:innen herauszuarbeiten, sie nach binären Kategorien wie Geschlecht oder Nationalität einzuteilen, miteinander zu vergleichen und mit oppositionellen (Kontroll-)Gruppen zu kontrastieren. Es interessiert uns auch nicht, ein sogenanntes >Milieu< unter dem Aspekt von Generation oder Geschlecht zu (re-)konstruieren und auf dieser Grundlage Erfahrungen oder Orientierungen zu kategorisieren. ${ }^{11}$ Mit Ordnungen und Kategorien zu arbeiten, affirmiert diese zumindest vorläufig und schließt Uneindeutigkeit aus. Bestenfalls 
werden Essentialisierungen, Homogenisierungen und Dichotomisierungen durch feinere oder neue ersetzt, nicht aber verflüssigt. Genau so funktionieren Rassismen, wie Hall eindrücklich argumentiert. ${ }^{12} \mathrm{Um}$ keine Rassismen und anderen gesellschaftlichen Machtverhältnisse zu reproduzieren, aber auch, um für die Pluralität und Ambiguität von Deutungs- und Handlungsmöglichkeiten offen $\mathrm{zu}$ bleiben, beziehen wir also Geschlecht, Alter und all die anderen machtförmigen Anrufungen in die Analyse ein und behandeln sie als gesellschaftliche Positionierungen, die sich materialisieren und bedeutsam werden können.

Für unser Forschungsprojekt sehen wir uns also vor der Herausforderung, einerseits der erneuten Rassifizierung von >Muslim:innen<, auch durch die Konstruktion neuer oder von Unter-Kategorien von >Muslim:innen $\mathrm{zu}$ widerstehen, andererseits aber die Deutungen und Handlungen im Umgang mit der rassifizierenden Adressierung als >Muslim:innen< herauszuarbeiten. Uns interessieren ganz konkret die Antworten auf die Anrufung. Insofern müssen wir die Anrufung >Muslim:in dem Sample zugrunde legen, ohne aber den Fehler zu begehen, die befragten Personen als Personenkategorie zu bestätigen: Eine Untersuchung zu Lehrkräften interviewt Lehrkräfte, eine Untersuchung zu Muslim:innen entsprechend Muslim:innen. Im Unterschied zum häufig vorgeschlagenen Vorgehen bei rekonstruktiven Forschungsmethoden entwickeln wir aber keine Subjekt- oder Gruppen-Kategorien und auch keine Binnen-Identitäten (Muslim:in-Typ a/b/c, muslimische Frauen der zweiten Generation). Vielmehr arbeiten wir Strategien heraus, die unter anderem mit der Adressierung als >muslimische Frau oder >türkischer Arbeiter zusammenhängen können, ohne sie als Typ darauf festzulegen und andere Aussagen vor diesem Hintergrund zu deuten bzw. andere Personen in diese Kategorien einzuordnen. Die Berücksichtigung ihrer Adressierung oder Selbstpositionierung dient in der konkreten Sequenz dazu, ihre Deutung bzw. ihre Strategie zu verstehen und bezieht jeweils jene Positionen, Kategorien, Machtverhältnisse usw. ein, die explizit oder implizit (an-)gedeutet werden. 
Unzusammenhängende oder widersprüchliche Aussagen einer Person oder eines >Typus` müssen also nicht als solche problematisiert und in feinere oder neue Kategorien überführt werden, um den Widerspruch aufzulösen und konsistente Typen zu generieren. Vielmehr ergeben sie unserer Vorgehensweise nach in einem spezifischen Kontext Sinn, etwa in der Konstellation der Diskussionsteilnehmer:innen, bezüglich des thematischen Fokus, wegen des intersektionalen Changierens, des Abwägens von Folgen und aus anderen Gründen, die es zu rekonstruieren gilt: $\mathrm{um} \mathrm{zu}$ verstehen, nicht um $\mathrm{zu}$ ordnen. Sie gehen also in die Interpretation der Argumentation ein, um die Deutungs- und Handlungsstrategien zu entschlüsseln und nicht, um einen Deutungsoder Handlungstyp zu (re-)konstruieren.

Wir sprechen unsere Gesprächsteilnehmer:innen also nicht analog zum hegemonialen Diskurs als >Muslim:innen monialen Unterscheidungen nach Alter, Geschlecht usw. an, sondern lassen offen, ob und gegebenenfalls in welcher Weise sie sich selbst oder auch die anderen Diskussionsteilnehmer:innen als Muslim:innen einordnen, und ob und in welcher Weise sie sich in den durch gesellschaftliche Machtverhältnisse hervorgebrachten Personenkategorien wiederfinden und darin positionieren. Unsere Fragestellung macht zudem deutlich, dass wir um die Markierung als sgefährliche Muslim:in wissen und diese Figur in der Forschungsfrage problematisieren (»Sicherheitsdiskurs«). Wir legen unser Erkenntnisinteresse offen und bitten unsere Gesprächspartner:innen, über ihre Sichtweisen zu sprechen (»wie nimmst du das wahr, wie siehst du das«), zu assoziieren und zu diskutieren, was sie an der Frage für wichtig halten. Durch die gezielte (anstelle der beliebigen oder natürlichen) Zusammensetzung der Gruppen versuchen wir theoriegeleitet, den im antimuslimischen Rassismus und insbesondere im Sicherheitsdiskurs angerufenen Positionen Rechnung zu tragen, sodass in den Diskussionen sowohl die Ambiguität als auch die Konkretheit sowie die Suchbewegungen zum Vorschein kommen. Das heißt, unser Forschungsdesign zielt nicht darauf, zur Ordnung der Dinge beizutragen, sondern im Gegenteil die Ordnung kritisch zu reflektieren, ihr Gefüge aufzubrechen, die Komplexität zum Vorschein kommen zu lassen und das zur unhinter- 
fragten Normalität Gewordene in Frage zu stellen. Insofern liegt der Fokus unserer Rekonstruktion des geteilten, aber auch differenten, kollektiven Wissens auf den ihnen zugrunde liegenden geteilten und differenten, kollektiven und spezifischen Erfahrungen, die durchzogen sind von interrelationalen, intersektionalen, gesellschaftlichen Machtverhältnissen, deren Relevanzen in der Auswertung zu berücksichtigen sind, um spezifische Deutungen und Strategien nachzuvollziehen.

\section{Nutzen und Grenzen rekonstruktiver Methoden}

Wir haben uns für die Gruppendiskussion als Erhebungsmethode und die Dokumentarische Methode als Auswertungsmethode entschieden, um einen Zugang zu kollektiven (impliziten und handlungsleitenden) Wissensbeständen von muslimischen Akteur:innen $\mathrm{zu}$ erhalten. Die Auswertung von Einzelinterviews mithilfe der Objektiven Hermeneutik hingegen dient der Rekonstruktion der diskursiven Struktur des Sicherheitsdiskurses, des Erfahrungswissens und der Handlungspraxis unserer Gesprächspartner:innen sowie ihren Subjektivierungsweisen und Selbsttechnologien im Umgang damit.

\section{Dokumentarische Methode: Kommunikatives, atheoretisches und situiertes Wissen}

Die Dokumentarische Methode eignet sich dazu, die Aushandlung von Deutungen und Umgangsweisen mit dem Sicherheitsdiskurs unter Muslim:innen zu untersuchen. In Gruppendiskussionen kann das kommunikative Miteinander mehrerer Personen und die Herstellungspraxis ihrer Wirklichkeit rekonstruiert werden. ${ }^{13}$ Laut Bohnsack, Przyborski und Schäffer bietet diese Methode die Möglichkeit, einen »Zugang zu kollektiven [H.i.O.] Erfahrungen und Orientierungen ${ }^{14} \mathrm{zu}$ erhalten.

\footnotetext{
13 Nohl et al. 2013: 21ff.

14 Bohnsack/Przyborski/Schäffer 2010: 13.
} 
Die in der vorliegenden Publikation vorgestellten Ergebnisse verweisen auf unterschiedliche Umgangsweisen mit dem Sicherheitsdiskurs durch muslimische und muslimisierte Akteur:innen. Konjunktive Erfahrungsräume sind gemeinsame Erfahrungen und Handlungspraxen (auch kollektive Deutungsmuster genannt), die Menschen zu einem spezifischen Thema teilen, ohne sich untereinander kennen $\mathrm{zu}$ müssen. Diese können an jenen Stellen der Gruppendiskussion erkannt werden, »wo diejenigen, die ein gemeinsames Problem oder allgemeiner: einen gemeinsamen [H.i.O.] Erlebnishintergrund oder existentiellen Hintergrund teilen, in ein darauf gerichtetes oder darauf aufbauendes kommunikatives Miteinander [H.i.O.] eintreten ${ }^{15}{ }^{15}$ Auch unsere Rekonstruktion zielt darauf, konkrete Handlungsstrategien herauszuarbeiten.

In Anlehnung an die Dokumentarische Methode fassten wir zunächst den thematischen Verlauf zusammen, wählten die für unsere Fragestellung relevanten Sequenzen aus, die sich durch eine besondere Gesprächsdichte auszeichnen, transkribierten sie und arbeiteten Oberund Unterthemen heraus. Daraufhin schrieben wir zu jeder Sequenz formulierende Interpretationen, auf deren Grundlage wir Diskursmodi identifizierten: antithetisch, parallel, oppositionell, univok und divergent. ${ }^{16}$ Die folgende Textsortentrennung dient dazu, das atheoretische vom kommunikativen Wissen zu unterscheiden. Beim kommunikativen Wissen handelt es sich um alltägliches Wissen. Es tritt in Schilderungen von Handlungen in Erscheinung und wird in Argumentationen und Bewertungen explizit geäußert (weil-Motive ${ }^{17}$ ). Dagegen wird das atheoretische Wissen in Erzählungen und Beschreibungen deutlich, es stellt »ein implizites oder still-schweigendes, ein konjunktives Wissen $\aleph^{18}$ dar. Es ist den Personen reflexiv nicht verfügbar und wird von den Forscher:innen rekonstruiert. Das Spannungsverhältnis zwischen den beiden Wissensformen spielt für die reflektierende Interpretation 
eine wichtige Rolle, um die Art und Weise herauszuarbeiten, wie die Themen bearbeitet werden.

Die gängige Unterscheidung beider Wissensformen kann jedoch aus unserer Sicht die unterschiedlichen Bezüge auf Wissensbestände aus einer rassifizierten oder unmarkierten Position nicht hinreichend berücksichtigen. Der Methodologie der Dokumentarischen Methode zufolge nimmt das kommunikative Wissen das öffentliche Wissen in den Blick und wird expliziert, während das atheoretische Wissen auf den Umgang damit verweist und implizit ist. Die Zuordnung zu dem einen oder anderen Wissen wird durch eine Textsortentrennung (s.o.) identifiziert. Diese Trennung vermag es aber im Zusammenhang mit Redebeiträgen von rassifizierten Personen nicht, ihre spezifische Situation zu berücksichtigen: (Als) Muslim:innen (Markierte) verfügen zwar über ein gemeinsames öffentliches Wissen, das sie mit Angehörigen des Rests der Gesellschaft teilen, jedoch stellt das »situierte Wissen « ${ }^{19}$ ebenfalls ein gemeinsames Wissen gleicher Struktur dar und muss mit berücksichtigt werden. Das bedeutet im Umkehrschluss, dass nicht nur marginalisierte Personen auf ein situiertes Wissen zurückgreifen, sondern dass auch das hegemoniale Wissen situiert ist, obwohl es sich als allgemein gültig gibt. Während die hegemonialen Wissensbestände bekannt sind, finden die Wissensbestände marginalisierter Personen kaum oder gar keinen Eingang in das sogenannte Alltagswissen, obwohl es ihnen selbst neben dem hegemonialen Wissen ebenfalls als

19 Donna Haraway spricht von situiertem Wissen und kritisiert, dass die westliche Kultur Objektivität und Wissenschaftlichkeit für sich beansprucht und hierdurch weiße Herrschaftsverhältnisse reproduziert, um sich zu legitimieren. Durch essentialistische und unkritische Argumentationsweisen schließt sie marginale Wissensbestände aus. Demgegenüber eröffnet eine Haltung, die die Situiertheit des Wissens, seine Verortung anerkennt, »die Perspektive solcher Blickwinkel anzustreben, die niemals im voraus bekannt sein können und die etwas sehr Ungewöhnliches versprechen, nämlich ein Wissen, das die Konstruktion von Welten ermöglicht, die in geringerem Maße durch Achsen der Herrschaft organisiert sind (Haraway 1995: 85). 
kommunikatives Wissen zur Verfügung steht. ${ }^{20}$ Dies wirkt sich auf die Rekonstruktionen dahingehend aus, dass wir berücksichtigen müssen, dass unsere Diskussionsteilnehmer:innen das gemeinsame und öffentliche Wissen kennen und sich darauf beziehen und gleichzeitig dieses von ihrem eigenen situierten Wissen differenzieren können. In der Artikulation des situierten, alltäglichen, kommunikativen Wissens der nicht-muslimischen Gesellschaft wiesen unsere Diskussionsteilnehmer:innen mitunter auf hegemoniale Wissensbestände hin, die sie als muslimisch Markierte homogenisieren und als Sicherheitsgefahr konstruieren. Die hegemonialen Wissensbestände werden von unseren Diskussionsteilnehmer:innen meist zitierend vorgetragen, explizit oder implizit reflektiert und der Kritik unterzogen. Gleichzeitig wird den hegemonialen Wissensbeständen anderes Wissen gegenüber gestellt, das ihre Perspektiven sichtbar macht und ihre Lebensrealitäten widerspiegelt. Wir identifizieren es daher als kommunikativ-situiertes Wissen von (als) Muslim:innen (Markierten) und bezeichnen es als Erfahrungswissen oder situiertes Wissen. Dieses Wissen wird von der nicht-muslimischen Gesellschaft nicht geteilt und ist ihr häufig auch gar nicht bekannt. Das kommunikativ-situierte Wissen von Muslim:innen ist kein allgemeines, alltägliches und kann nicht als Common-Sense der Gesamtgesellschaft vorausgesetzt werden, obwohl es als solches zur Verfügung steht. Dörner et al. weisen darauf hin, dass durch einen "Common-Sense-Blick « ${ }^{21}$ bestimmte Phänomene unberücksichtigt bleiben. Das kommunikativ-situierte Wissen unserer Diskussionsteilnehmer:innen ist als Erfahrungswissen von Alltagstheorien nicht gedeckt, sodass darauf in der Forschungspraxis ohne die von uns hier beschriebenen Modifikationen im methodischen Vorgehen nicht zurückgegriffen werden kann.

20 Indigene, Schwarze und Forscher:innen of Color haben auf ein solches Wissen hingewiesen (Denzin/Lincoln/Smith 2008; Collins 1993; Lewis 1996) und verdeutlicht, dass z.B. marginalisierte Erfahrungen und Wissensbestände sehr wohl bestehen, aber de-legitimiert werden und damit noch keinen Eingang in den eurozentrischen Wissenskanon gefunden haben. 


\section{Objektive Hermeneutik: Objektive Bedeutungsmuster und latente Sinnstrukturen}

Anknüpfend an die Rekonstruktionsergebnisse der Dokumentarischen Methode haben wir ausgewählte Personen zu unseren Einzelinterviews eingeladen, um unser Verständnis der identifizierten Umgangsweisen mit dem Sicherheitsdiskurs zu vertiefen. Zirkulär vorgehend haben wir hierfür nach und nach Personen eingeladen und zunächst dieselbe Einstiegsfrage gestellt, jedoch die Nachfragen an den Personen und ihren beruflichen und/oder aktivistischen Erfahrungen orientiert.

Die Objektive Hermeneutik eignet sich zur Rekonstruktion der unbewussten bzw. latenten Handlungsmöglichkeiten und ihrer zugrunde liegenden Bedeutungen, auf die (als) Muslim:innen (Markierte) im Umgang mit dem Bedrohungsszenario zurückgreifen. Im Zuge der Anwendung der Methode stellten wir allerdings fest, dass sich die transkribierten Interviews als »Protokolle [H.i.O.] der Wirklichkeit ${ }^{22}$ nicht ausschließlich »entlang geltender Regeln $\ll^{23}$ von allen Interpret:innen verstehen lassen. Vor dem Hintergrund unseres Forschungsvorhabens und rassismuskritischer Theorien gehen wir davon aus, dass Gesellschaften von rassistischen Strukturen durchzogen sind und jedes Mitglied einer Gesellschaft darin verstrickt ist, sodass sich sowohl weiße als auch rassifizierte Personen Strategien zum Umgang damit aneignen. ${ }^{24}$ Die unterschiedlichen Weisen des Verstricktseins in rassistische Machtverhältnisse bringt es mit sich, dass nicht angenommen werden kann, dass das Wissen zu Rassismus ein Regelwissen darstellt und die Interpret:innen zwangsläufig darüber verfügen, sodass sogenannte übersubjektive Strukturen bzw. Latenzen herausgearbeitet werden können.

In Anlehnung an sinnverstehende Wissenschaften geht die Objektive Hermeneutik davon aus, »dass sich die sinnstrukturierte Welt durch Sprache konstituiert und in Texten materialisiert «. ${ }^{25}$ Die Welt

\footnotetext{
22 Wernet 2009: 12.

23 Ebd.: 18.

24 Mecheril 1994: 57ff.

25 Wernet 2009: 11.
} 
ist demnach durch Regeln vorstrukturiert und das soziale Handeln orientiert sich an den bestehenden Regeln. Für die Interpretation ist die Berücksichtigung und Einbeziehung dieser Regeln relevant, damit die Rekonstruktion sozialer Praxis gelingt. Im Mittelpunkt der Interpretation stehen also die Alltagshandlungen der Gesprächspartner:innen, die dahingehend analysiert werden, welche unbewussten bzw. latenten Regeln und Strukturen ihrem Handeln zugrunde liegen. Diese Regeln und Strukturen sind objektiv vorhanden und universal, sodass »die fundamentale Bedeutung der Regelgeleitetheit [...] in ihrer Nicht-hintergehbarkeit [besteht]. Die Lebenspraxis kann sich ihr weder entziehen, noch kann sie die Regelgeltung außer Kraft setzen ${ }^{26}{ }^{26}$ Wenn aber die Welt aus viel komplexeren, widersprüchlicheren und vielschichtigeren Regeln besteht, wie dies etwa durch unterschiedliche Positionen, Normalitäten, Regeln und Ressourcen aufgrund von Rassismus und anderen Machtverhältnissen der Fall ist, so stellt sich die Frage, ob bzw. wie sie mit dieser Methode eingefangen werden können. Die Erfahrungen von (als) Muslim:innen (Markierten) stellen eine spezifische gesellschaftliche Wirklichkeit dar, die sich unter anderem durch die Erfahrung von (intersektionalem antimuslimischem) Rassismus von der sozialen Wirklichkeit anderer Gesellschaftsmitglieder unterscheidet und nicht mithilfe sogenannter universaler Regeln rekonstruiert werden kann. Vielmehr bedarf es zur Anwendung von Regeln eines fundierten Wissens über die Funktionsweisen von Rassismus, um regelgeleitet vorgehen $\mathrm{zu}$ können. Andernfalls gehen die unreflektierten, rassistischen Wissensbestände als latente Sinnstrukturen leitend in die Interpretation ein, sodass (als) Muslim:innen (Markierte) und ihre Alltagshandlungen als anders, fremd und bedrohlich affirmiert werden. Sich an einer sogenannten universalen Sprache für den deutschsprachigen Kontext zu orientieren, bedeutet also auch, die Interpretationen entlang der hegemonialen Konstruktion von >Muslim:innen vorzunehmen. Für die Rekonstruktion ihrer Handlungspraxis erscheint es demgegenüber relevant, ihre 
Rassismuserfahrungen und die im hegemonialen Wissen ausgeblendeten Lebensrealitäten mitzudenken und generell im Regelwissen das Mitlaufen von Rassismus, also Rassismus als Subtext, Kontext oder Haupttext, mit einzubeziehen.

Eine solche Interpretation ermöglicht es, die Auswahl der Handlungsmöglichkeiten (also die Selektivität des Handelns) von (als) Muslim:innen (Markierten) vor dem Hintergrund eines rassistischen Regelwissens $\mathrm{zu}$ interpretieren und $\mathrm{zu}$ rekonstruieren. Leanza schlägt vor, die rekonstruierten Fallstrukturen der Kritik zugänglich zu machen und auch die sichtbar gemachten latenten Sinnstrukturen »als Selektion aus einem Raum von Möglichkeiten « $\mathrm{zu}$ verstehen, sodass »bestimmte Implikate von Deutungen oder aber auch versteckte Herrschafts- und >Entfremdungsstrukturen « aufgedeckt werden ${ }^{27}$ können.

Anknüpfend an diesen Vorschlag bezogen wir die Kritik bereits in den Interpretationsprozess ein und unterzogen die objektiven Bedeutungsstrukturen $^{28}$ einer rassismuskritischen Reflexion. Die objektiven Bedeutungsstrukturen sind von rassistischem Alltagswissen durchzogen, hegemoniale Diskurse beziehen sich auf antimuslimische Stereotype als unhinterfragte Wissensbestände. ${ }^{29}$ Unseren Gesprächspartner:innen sind diese hegemonialen Diskurse bekannt, ohne dass sich ihr Handeln ausschließlich daran orientiert. Ihre Praxen, Perspektiven und Wissensbestände gehen in den hegemonialen Diskursen nicht auf, sie werden in der Regel nicht gesehen und gehört, sondern missrepräsentiert und missgedeutet, sodass es dazu kein Regelwissen

27 Leanza 2008: 103.

28 Objektive Bedeutungsstrukturen meint in der Methodologie der Objektiven Hermeneutik, dass jedes Mitglied einer Gesellschaft für seine Handlungen auf bestehende Strukturen und Regeln, die bereits bestehen, zurückgreift. Diese Strukturen und Regeln eignet sich der Mensch im Laufe seiner Sozialisation an. Das Ziel der Rekonstruktion liegt darin, diese herauszuarbeiten, sowie die Bedeutung der Auswahl von Handlungsmöglichkeiten zu verstehen (Wernet 2009: 13ff.).

29 Attia 2009: 53-94. 
gibt. Um sprechen zu können bzw. um gehört zu werden, orientieren sich unsere Gesprächspartner:innen bis zu einem gewissen Grad trotzdem an Themen, Begriffen, Metaphern und Sprachmotiven aus dem hegemonialen Islamdiskurs. Auf diese Weise können sie sich zunächst verständlich machen. Dies gilt auch für das Gespräch unter (als) Muslim:innen (Markierten), da sie miteinander bisweilen auch auf der Grundlage des hegemonialen Diskurses sprechen und dieser ihre geteilte Wirklichkeit (mit-)konstruiert. Aufgrund der rassismuskritischen Ausrichtung unserer Studie und der rassismuskritisch und intersektional informierten Interpretationsgruppen war es uns möglich, das Erfahrungswissen unserer rassifizierten Gesprächspartner:innen zu erkennen und die latenten Sinnstrukturen und Bedeutungen ihrer Lebenspraxen zu rekonstruieren. Insofern war es uns möglich, auch jene Strukturen, Erfahrungen und Wissensbestände zum Vorschein zu bringen, die nicht durch den Text und die ausgesprochenen Worte repräsentiert werden. Vielmehr erhielt das Gesagte vor dem Hintergrund von rassismusbezogenem Wissen, Theorien und Reflexionen seine Bedeutung.

Insofern konnten wir »die Selbstauffassungen des Handlungssubjekts, die Handlungsintentionen $\aleph^{30}$ vor dem Hintergrund des antimuslimischen Rassismus herausarbeiten. Die methodischen Schritte ${ }^{31}$ ließen sich problemlos anwenden und gewährleisten, dass die Lebenspraxis in ihrer Struktur rekonstruiert werden kann. Hierdurch lassen sich mehrere Umgangsstrategien und Subjektivierungsweisen herauskristallisieren. Wir fassten Gemeinsamkeiten mehrerer Einzelinterviews zusammen und stellen sie als Fallstruktur-Generalisierungen vor. Darunter versteht Wernet, dass

30 Wernet 2009: 18.

31 Oevermann versteht die Interpretation mithilfe der Objektiven Hermeneutik, als deren Begründer er gilt, als »Kunstlehre« (Oevermann 1993: 126) und gibt für die praktische Anwendung keine expliziten Vorgaben. Wernet hingegen hat in seinem Standardwerk Einführung in die Interpretationstechnik der Objektiven Hermeneutik»methodentechnische [...] Kernoperationen «(Wernet 2009: 10) für die Interpretation vorgestellt und diese anhand eines Lehrer:inneninterviews praktisch angewendet (ebd.: $52 \mathrm{ff}$.). 
»[d]er analysierte Fall [...] immer schon allgemein und besonders zugleich [ist]. Denn in jedem Protokoll sozialer Wirklichkeit ist das Allgemeine ebenso mitprotokolliert wie das Besondere im Sinne der Besonderheit des Falls. Der konkrete Fall ist insofern schon mehr als ein Einzelfall, als er ein sinnstrukturiertes Cebilde darstellt $«{ }^{32}$

In unserer Studie verstehen wir unter einem Fall Deutungen und Strategien. Folglich stellen wir die Fallstrukturgeneralisierungen jedes Einzelinterviews nicht einzeln vor, sondern fassen sie thematisch zusammen und stellen daher unterschiedliche Umgangsweisen mit dem Bedrohungsszenario vor.

\section{Schlussfolgerung}

Vor dem Hintergrund unserer Erfahrungen mit den genutzten rekonstruktiven Forschungsmethoden erscheint ihre macht- und rassismuskritische Anpassung geboten, sobald die Erfahrungen und Wissensbestände rassifizierter Personen interpretiert, analysiert und rekonstruiert werden. Wir halten dies auch für Forschungsfragen für angebracht, die nicht auf Rassismuserfahrungen fokussieren, da diese immer mitlaufen und relevant sein könnten. Bereits durch den groben Umriss unseres Forschungsinteresses und die Auswahl der Teilnehmer:innen an der Gruppendiskussion, deren Wissen um ihre eigenen Expertisen, denen der anderen und auch um unsere Expertisen in einem spezifischen Fachgebiet, geben wir einen bestimmten Rahmen vor. Diesen versuchen wir nicht zu kaschieren, indem wir etwa die Adressierung von (als) Muslim:innen (Markierten) zu vermeiden versuchen oder das Thema unserer Forschung vage halten. Vielmehr sprechen wir die Teilnehmer:innen explizit auf genau jene Adressierung an und rufen sie als Expert:innen ihrer selbst auf, halten uns aber in der Gruppendiskussion mit eigenen Beiträgen zurück und verbleiben in der Moderationsrolle. 
Uns interessieren sowohl explizite Äußerungen unserer Diskussionsteilnehmer:innen angesichts ihrer Erfahrung mit dem antimuslimischen Rassismus (kommunikatives Wissen) als auch jene handlungsleitenden, kollektiven (widerständigen) Wissensbestände, die sich konjunktiv artikulieren und der indirekten, gouvernementalen Selbstführung in Form alltäglicher Handlungen Rechnung tragen. Es geht uns also darum, die Wissensbegriffe in Dialog zu setzen, anstatt sie scharf voneinander zu trennen. Die Dokumentarische Methode schlägt dafür das Konzept des Orientierungsrahmens vor und verweist auf das Spannungsverhältnis beider Wissensformen. Sie geht davon aus, dass nur jene Normen, die im Kontrast zum eigenen Handeln erkannt werden, explizit formuliert werden können und deshalb kommunikativ sind, denn sonst würde die Norm in den Habitus übergehen und sich konjunktiv manifestieren. Nur jene Normen, die von Subjekten aktiv wahrgenommen werden, finden dementsprechend Eingang ins kommunikative Wissen, während sich im atheoretischen Wissen die impliziten, handlungsleitenden Wissensbestände befinden, da diese im konjunktiven Erfahrungsraum entstehen.

Vor dem Hintergrund intersektionaler, rassismuskritischer Theorien verstehen wir das üblicherweise als kommunikatives Wissen Bezeichnete als hegemoniales Wissen und meinen hiermit jenes öffentliche Wissen, das allen Gesellschaftsmitgliedern und damit auch Muslim:innen bekannt ist und im Grunde das situiert-kommunikative Wissen der Dominanzgesellschaft darstellt. Mit Erfahrungswissen oder situiertem Wissen meinen wir jenes kommunikativ-situierte Wissen unserer Diskussionsteilnehmer:innen, das ihnen darüberhinaus aufgrund ihrer Muslimisierung bekannt ist. Diese Spezifizierung eröffnet uns erst die Möglichkeit, unterschiedliche konjunktive Erfahrungsräume und damit unterschiedliche Umgangsweisen mit dem Sicherheitsdiskurs herauszuarbeiten. Sie basieren auf der Erfahrung des antimuslimischen Rassismus und dem Umgang mit dem Bedrohungsszenario und stellen damit die soziale Wirklichkeit von (als) Muslim:innen (Markierten) in einer Gesellschaft dar, die durch Rassismus strukturiert ist. 



\section{Ver_Handlungen}

In drei Gruppendiskussionen ging es uns darum, eine Breite an Deutungen und Strategien zu generieren, und nicht, wie sonst in Gruppendiskussionsverfahren üblich, Personenkategorien oder Organisationstypen zu konstruieren. Gruppendiskussionen bieten sich dennoch für unsere Fragestellung an, weil im Gespräch mit anderen Reflexionsprozesse verbal zugänglich und in der Explikation und der Aushandlung von Positionen entwickelt und differenziert werden. Wir luden Personen zur Diskussion ein, die in Vereinen, Kollektiven, Verbänden und anderen Zusammenschlüssen aktiv sind. Alle Diskussionsteilnehmer:innen äußern sich entweder selbst als Muslim:innen oder werden als solche adressiert. Die Wahl fiel auf Personen, die auf einen breiten Fundus an Deutungs- und Handlungsmustern zurückgreifen können.

Zur ersten Gruppendiskussion waren der Einladung Vertreter:innen von Moscheegemeinden und anderen Verbänden gefolgt. Im Zuge der Interpretation der ersten Sequenz stellte sich uns die Frage, welche Relevanz es haben könnte, wenn die Verbände auf konkrete eigene Restriktionen im Zusammenhang mit dem Sicherheitsdiskurs zurückblicken und von konkreten Auswirkungen des sicherheitspolitischen Disziplinar- und Sanktionssystems berichten können. Diesen Aspekt fokussierten wir in der zweiten Gruppendiskussion und luden entsprechend ein. In der dritten Gruppendiskussion interessierte uns, welche Deutungen und Strategien diskutiert werden, wenn die Teilnehmer:innen nicht als Vertreter:innen von Verbänden oder Moscheegemeinden sprechen, sondern sich stärker auf ihre aktivistische, 
künstlerische oder akademische Arbeit beziehen und daran orientiert zu Wort melden.

Die Diskussionen eröffneten wir mit der Bitte, miteinander darüber zu sprechen, wie die Diskussionsteilnehmer:innen den Sicherheitsdiskurs wahrnehmen, welche Auswirkungen er auf sie hat und wie sie damit umgehen. Zur Auswertung nutzten wir die Dokumentarische Methode als Werkzeug und passten sie unserer Fragestellung sowie unseren erkenntnistheoretischen und forschungsethischen Positionen an. Alle drei Gruppendiskussionen erhielten von uns die gleichen Informationen und den gleichen Impuls: »Wir würden Euch gerne bitten, miteinander darüber zu sprechen, wie Ihr den Sicherheitsdiskurs wahrnehmt, was er für Euch bedeutet und wir Ihr damit umgeht. Ihr könnt anfangen, wie Ihr wollt, wir werden uns nicht am Gespräch beteiligen.« Zwei Audiogeräte zeichneten die Diskussion auf, zudem protokollierten zwei Forscher:innen ihre Wahrnehmungen. An den Gruppendiskussionen nahmen die folgenden Personen (Pseudonyme) teil:

G 1: Aslı A., Mohamed B., Sidar C., Gülçin D., Viola E. und Rahman F. (96 Minuten)

G 2: Rania G., Samir H. und Abdul I. (84 Minuten)

G 3: Tasnim ]., Fatouma K., Leyla L., Hassan M. und Asmah N. (127 Minuten)

Die folgenden drei Abschnitte fassen unsere Forschungsergebnisse aus den Gruppendiskussionen zusammen. Die Fokussierung auf die genannten Aspekte und die Spannung zwischen den jeweiligen Polen Inter_Sektionen, Ent_Solidarisierung und Ver_Antworten entwickelten wir induktiv aus dem Material. Der Datenerhebung und -auswertung gingen theoretische, ethische und methodologische Auseinandersetzungen voran. Sie begleiteten den Forschungsprozess und ragen in Form neuer Erkenntnisse und Fragen an Theorien sowie Forschungsmethoden und -ethik darüber hinaus. 


\section{Inter_Sektionen}

In den Gruppendiskussionen greifen die Teilnehmer:innen ihre Anrufung als `Muslim:innen auf. Sie sprechen als Muslim:innen, vervielfältigen und verkomplizieren aber ihr Muslimischsein, indem sie es pluralisieren und mit weiteren Positionierungen kreuzen. Damit weisen sie einerseits die dem antimuslimischen Rassismus inhärente Essentialisierung und Homogenisierung >der Muslimin und sdes Muslims zurück, greifen aber andererseits auf jene Subjektpositionen zurück, die den antimuslimischen Rassismus im Allgemeinen und den Sicherheitsdiskurs im Besonderen durchziehen. Narrative zu Orient und Islam sind in der Konstruktion des fremden, rückständigen und bedrohlichen Anderen intersektional mit Geschlecht, Sexualität, Klasse, Bildung, Nationalität, Migration, Kultur, Ethnizität, Sprache, Hautfarbe und Religiosität verwoben. Während die Intersektionen in orientalisierenden und antimuslimischen Diskursen das Fremde signifizieren, vermag es ihre Thematisierung als Machtverhältnisse, die Entitäten zu verflüssigen und in konkreten Situationen zu immer wieder neuen Konstellationen zu verweben, ohne beliebig zu werden. Das eröffnet nach sinnen und nach saußen< hin Ansatzpunkte zur Kritik an Essentialisierung, Homogenisierung, Dichotomisierung und Hierarchisierung.

In beiden Fällen bewegt sich der Bezug auf die genannten Differenzkategorien und Machtverhältnisse innerhalb des hegemonialen Diskurses, wenn auch in unterschiedlichen Weisen, die unterschiedliche Formen der Unterwerfung und des Widerstands aufweisen. Die Diskussionsteilnehmer:innen antworten zum Beispiel auf ihre Anrufung als Muslim:innen, indem sie ihre Adressierung als Hijabi, >Gastarbeiter<, >dritte Generation< usw. aufgreifen und in den Diskussionen auch untereinander ihr Muslimischsein mit Bezug auf diese Anrufungen artikulieren. Im Zuge der Performativität von Intersektionen kommt es zu Verschiebungen und Affirmationen des Diskurses sowie zu vielfältigen und wechselnden Bezugnahmen aufeinander, die implizit oder explizit intersektional eingeleitet und gerahmt werden. Indem die Gesprächspartner:innen die Anrufung annehmen und durch sie sprechen, äußern sie sich als informierte Bürger:innen 
dieser Gesellschaft ${ }^{1}$, denen die mit dem Islam- und Sicherheitsdiskurs verwobenen Intersektionen geläufig und vertraut sind. Sie setzen sie ein, reflektieren sie und verweben sie gegebenenfalls neu.

Im Folgenden fragen wir danach, wie in den Diskussionen Positionierungen und Intersektionen sichtbar gemacht und verhandelt werden. Dies erfolgt mit Bezug auf unterschiedliche Machtverhältnisse. Die Diskussionsteilnehmer:innen reflektieren, wie sie in der Gesellschaft als intersektional positionierte Muslim:innen adressiert werden und welche Spielräume sie angesichts der intersektionalen Durchdringung des Sicherheitsdiskurses ergreifen können. Sie argumentieren vor dem Hintergrund ihrer pluralen, sich kreuzenden und wieder auseinanderlaufenden Positionierungen und deren Bedeutungen in ihrem privaten, beruflichen und zivilgesellschaftlichen Alltag. Es wird deutlich, wie sie mit ihrer intersektionalen Anrufung als >Muslim:innen $<$ in konkreten Situationen umgehen. Indem sie die Gemeinsamkeiten und Unterschiede untereinander in die Diskussion einführen, können die Diskussionsteilnehmer:innen mit den jeweiligen Anrufungen verbundene Problematisierungsweisen bezüglich sicherheitspolitischer Themen herausarbeiten. Die Gruppendiskussionen dienen hierzu nicht nur als Reflexionsraum, sondern führen in actu vor, dass unterschiedliche Erfahrungen zu unterschiedlichen Einschätzungen und Handlungsoptionen beitragen können, aber expliziert werden müssen, um miteinander sprechen zu können.

Intersektionale Machtverhältnisse sind im Verlauf der Diskussionen, in den Reflexionen zwischen den Diskussionsteilnehmer:innen und in unseren Rekonstruktionen vielfach entscheidend. Die Diskussionsteilnehmer:innen rufen Differenzen untereinander entlang der genannten Kategorien und Verhältnisse auf und weisen explizit auf intersektionale Gemeinsamkeiten und Unterschiede hin, um sich in der Diskussion voneinander abzugrenzen oder um gemeinsame Positionen $\mathrm{zu}$ begründen. Sie re-zentrieren ihr Muslimischsein in Interrelation $\mathrm{zu}$ anderen Subjektpositionen, sodass differenzierte Reflexionen ihrer Erfahrungen, Positionen und Praktiken im Zusammenhang mit dem 
Sicherheitsdiskurs und dem antimuslimischem Rassismus möglich werden.

Die Diskussionsteilnehmer:innen tauschen sich über ihre Rassismuserfahrungen im Zusammenhang mit dem Sicherheitsdiskurs aus und identifizieren über die Gemeinsamkeiten hinweg auch Unterschiede in ihrem Muslimischsein als gelebte Erfahrungen und Positionierungen. Unter Berücksichtigung ihrer intersektional-muslimischen Bezüge ist es den Teilnehmer:innen möglich, ihre Erfahrungen in Relation zu setzen und teils in Nuancen, teils aber auch wesentlich zu unterscheiden und in ihrer Pluralität, Widersprüchlichkeit und Ambiguität als gemeinsame, nämlich vielstimmige muslimisch-deutsche Erfahrung zu reflektieren. Die Betroffenheit von antimuslimischem Rassismus vereint (als) Muslim:innen (Markierte) und kann im Rahmen der Analysen sowohl als explizites als auch als implizites Wissen rekonstruiert werden, das sowohl auf ihrer Kenntnis des hegemonialen Wissens als auch auf ihrem Erfahrungswissen beruht. Vor dem Hintergrund ihrer intersektionalen Rassismuserfahrungen ist es den Diskussionsteilnehmer:innen möglich, differenzierter und vertiefter über deren Effekte und über ihre Handlungsoptionen zu reflektieren.

\section{"Herr Sheikh abi»}

Der gegenderte antimuslimische Rassismus spielt im Kontext der Diskussionen zum Sicherheitsdiskurs wiederholt eine herausragende Rolle. Gülçin D. weist in der ersten Gruppendiskussion auf verschiedene Subjektpositionierungen hin, die sie als Adressierung und als Identifizierung ${ }^{2}$ artikuliert und verhandelt. Sie bezeichnet sich als Teil der »deutschtürkischen Muslime« und führt ihre Position als Frau, Mutter und Tochter ins Gespräch ein. Sie bezieht sich intersektional auf diese Positionierungen und tut dies in der folgenden Sequenz im Disput mit und in Abgrenzung von Rahman F., der aus der Perspektive eines Imams spricht. Im weiteren Verlauf der nun folgenden Rekonstruktion 
wird Gülçin D. sein (Rahman F.s) höheres Alter und ihre Zugehörigkeit zu unterschiedlichen Migrationsgenerationen betonen. ${ }^{3}$

Die Sequenz beginnt damit, dass Rahman F. von Nachbarschaftskonflikten berichtet und argumentiert, dass die Verstrickung der »Bürger « in antimuslimisch-rassistische Diskurse von der Medienberichterstattung herrührt. Seine Rede wirbt für Verständnis und Dialog und räumt eine Mitschuld von »unserer Seite« ein. ${ }^{4}$ Gegen diese Thematisierung legt Gülçin D. vehement Widerspruch ein. Sie rahmt ihre Argumentation intersektional und ruft bereits in der einleitenden Ansprache gleich mehrere Differenzdimensionen auf: »((stöhnt und atmet tief ein)) Herr Sheikh abi«.

Die Namen, die Gülçin D. wählt, sprechen auf mehreren Ebenen ihre Beziehung zu Rahman F. an: Der deutschen, bürgerlichen Benennung >Herr < folgt seine Adressierung als religiöse Autorität >Sheikh`, um schließlich mit der familiären Anrede für eine ältere Respektsperson sabi< zu enden. Im Unterschied zu der distanzierten Anrede >Herr greift Gülçin D. mit >Sheikh und sabi< auf Namen zurück, die eine Beziehung aufrufen. Sie deuten auf eine Beziehungskonstellation hin, in der Gülçin D. zunächst der religiösen Autorität Respekt zollt, um Rahman F. gleich darauf durch die familiäre Ansprache wieder näher an sich heranzurücken. Die verschiedenen Bezeichnungen weisen auf die Komplexität ihrer Beziehung hin, die aus Gülçin D.s Perspektive den Alters-, Migrations- und Generationsunterschied in Intersektion mit seiner religiösen Position hervorheben. Rahman F. gehört nicht ihrer »Wir«Gruppe, der Gruppe der »deutschtürkische[n] Muslime«, an. ${ }^{5}$ Gülçin D. erkennt seine Autorität an und würdigt seine religiöse Expertise. Ihre eigene Expertise wiederum möchte sie als die einer in Deutschland geborenen und sozialisierten Person anerkannt wissen. Sie erklärt Rahman F. geduldig-genervt, ((stöhnt und atmet tief ein)), in welcher Weise adäquat mit dem antimuslimischen Rassismus in Deutschland umzugehen ist. Geschlecht als weiteres in beider Namen impliziertes

3 Seine Selbstpositionierung wird weiter unten rekonstruiert.

4 Der Konflikt selbst wird im Abschnitt Ent_Solidarisierung interpretiert.

5 Auf diesen Aspekt wird im Abschnitt Ent_Solidarisierung näher eingegangen. 
Verhältnis spielt in der Beziehung zwischen Gülçin D. und Rahman F. in dieser Sequenz nur insofern eine Rolle, als dass die antimuslimischorientalistischen Adressierungen vergeschlechtlichend zwischen ihnen unterscheiden, von ihnen aber zunächst nicht geltend gemacht werden.

Gülçin D. erfährt von den anderen Diskussionsteilnehmer:innen Zustimmung durch Kopfnicken und Zwischenrufe wie: »Ich gebe ihr eigentlich Recht."Sie äußern ihre inhaltliche Zustimmung zu Gülçin D. und damit die Distanz zu Rahman F.s Argumentation in einem höflichen Ton (»eigentlich«). Aufgrund der Dynamik zwischen Gülçin D. und Rahman F. finden die anderen Diskussionsteilnehmer:innen jedoch keinen Zugang zum Gespräch in dieser Sequenz.

Gülçin D. berichtet davon, wie Lehrer:innen ihrer Tochter von ihr als »türkische[r] Mutter« bei schulischen Anlässen erwarten, »Börek« $\mathrm{zu}$ backen. Bereits ihre Mutter hat mit »Sarma« und »Börek« »die ganzen Nachbarn Jahrze::hnte verso::rgt«. Inzwischen folgt Gülçin D. der Aufforderung nicht mehr: »N::EIN, ich bring einen Apfelsaft mit! «. Sie lehnt den ihr zugewiesenen Platz als Fremde und als Frau entschieden ab: »Ich hab die Nase voll davon! « Damit weist sie gleichzeitig ihre Orientalisierung und ihre Vergeschlechtlichung zurück: Der »Apfelsaft« symbolisiert ihre Absage sowohl an den Arbeitseinsatz für ein aufwändiges Gericht, das Frauen in die Küche verweist, als auch an die Einverleibung des Exotischen, die sich der >Gastfreundlichkeit $<$ und der >orientalischen Küche bedient.

Gülçin D. setzt der intersektionalen Anrufung ihre Selbstpositionierung als Mitglied der Gesellschaft entgegen: »Ich bin hier groß geworden. Ich bin hier geboren. Ich bin ein Teil in dieser Gesellschaft. « Darin scheint sowohl die Dethematisierung von Gender als auch die Fokussierung auf (geschlechtsneutrale) Bürger:inschaft durch. Als Grund dafür, nicht mehr "nett« und »freundlich« auf ihre Adressierung als Fremde reagieren $\mathrm{zu}$ wollen, führt sie zudem an, dass sie auch dann keine gleichwertige Anerkennung erfährt, wenn sie sich der Anrufung unterwirft. Stattdessen wird sie weiterhin diskriminiert: »[U]nd bei einer

6 Auf diese Passage wird weiter unten näher eingegangen und im Abschnitt Ent_Solidarisierung Bezug genommen. 
kleinen Sache bist du trotzdem der Muslim. Ich WILL das nicht mehr machen. «Gülçin D. stellt in ihrer Argumentation nicht einfach eine Erfahrung einer anderen gegenüber - sie reflektiert vielmehr die Gleichzeitigkeit und Interrelation verschiedener Machtverhältnisse. Die Zitation der männlichen Anrede für sich selbst (»der Muslim«) wiederum verschiebt den Fokus ihrer Stereotypisierung. Ihr widerständiges Verhalten gegen ihre Adressierung als untergeordnete »türkische Mutter« wird mit ihrer Adressierung als bedrohlicher »Muslim« beantwortet. In beiden Konstellationen bleibt Gülçin D. fremd. Gleichwohl ändert sich ihre untergeordnete feminisiert-orientalistische Platzierung (gastfreundliche »türkische Mutter«) in eine übermächtige maskulinisiertmuslimisierte (bedrohlicher »Muslim«).

\section{"Story of our Life einfach nur"}

Die Sequenz ${ }^{7}$ wird von Hassan M. eingeleitet, der eingesteht, dass er nach seiner Migration als Erwachsener nach Deutschland »unbewusst« lange Zeit kein arabisches Buch in öffentlichen Verkehrsmitteln gelesen hat. Es entsteht eine dichte Gesprächsatmosphäre, in der die restlichen Diskussionsteilnehmer:innen zu erkennen geben, dass sie sich in der Schilderung wiedererkennen: ((murmelnd)) Oh mein Gott, Story of our Life einfach nur ((lacht)).«Ohne dies explizit auszusprechen, teilen die Diskussionsteilnehmer:innen das situierte Wissen, wonach hier die Rede von antizipierter und erlebter Rassismuserfahrung ist, die im Sicherheitsdiskurs besondere Relevanz erfährt. Das Wissen ist den Redebeiträgen inhärent und den Diskussionsteilnehmer:innen reflexiv verfügbar. Es gehört zu ihrem Alltagswissen und muss nicht ausgeführt, sondern kann als situiertes Wissen vorausgesetzt werden. In der rekonstruierten Sequenz diskutieren die Teilnehmer:innen nicht die Situation als solche und halten sich auch nicht mit möglichen Motiven der anderen Fahrgäste und deren Reaktionen darauf auf. Sie können diese jedoch nicht ignorieren und müssen sich darauf beziehen, auch wenn

7 Die Bedeutung von Migration in Intersektion mit dem Sicherheitsdiskurs wird weiter unten herausgearbeitet. 
sie sich mehr für ihre eigenen Praktiken und Gefühle interessieren. Die Variationen der Grunderzählung sind von intersektionalen Positionierungen durchzogen.

Leyla L. aus der dritten Gruppendiskussion trägt Hijab und problematisiert den gegenderten antimuslimischen Rassismus. Sie liest "Qur'an and Woman" in der U-Bahn und befürchtet, dass das Buch falsch gedeutet wird. Sie »schäm[t]« sich und hat »Angst«, dass »die Leute denken«, es ist "gut«, dass sie sich mit »Frausein im Islam, weil is ja problematisch « befasst. Auch hier führt die Rednerin nicht weiter aus, was »die Leute« meinen und wie sie zu dieser Einschätzung kommen, weil die Gesprächspartner:innen ein gemeinsames Erfahrungswissen hierüber teilen. Es ist auch nicht nötig, das namentlich genannte Buch näher vorzustellen, da es seit einiger Zeit in muslimischen Communities ein Bestseller ist. Leyla L. setzt voraus, dass die Gruppe sowohl das Buch einzuordnen weiß als auch ihr Dilemma nachvollziehen kann. Sie geht auch davon aus, dass das Buch in öffentlichen Verkehrsmitteln von der breiten Masse der Fahrgäste nicht erkannt wird. ${ }^{8}$ Das Wissen um den gegenderten antimuslimischen Rassismus reflektierend kann Leyla L. ihr Buch jedoch nicht »in Ruhe« lesen. $\mathrm{Zu}$ sehr befürchtet sie, dass sie den Diskurs sunterdrückte muslimische Frau bedient, wenn sie als Hijabi ein Buch zu Islam und Frauen liest. Sie möchte »einfach irgendein Buch« lesen, den Titel "Qur'an and Woman« nennt sie nur, weil er auf das Dilemma hindeutet.

Hassan M. kann auf die Erwähnung eines Titels gänzlich verzichten, hier ist es offensichtlich, dass es um »irgendein Buch« geht. In seiner Erzählung weist »Arabisch « auf sein Dilemma als >muslimischer Mann hin. Während er die Zeichen aufruft, die ihn im hegemonialen Islamdiskurs, zumal mit Fokus auf den Sicherheitsdiskurs, als >Gefährder markieren, ruft Leyla L. jene auf, die sie als sunterdrückte Frau deuten. Beide setzen sich unfreiwillig und auch widerstrebend mit ihrer Anrufung als >Muslim:innen a auseinander und reflektieren in ihrer

8 Wadud, Amina (1999): Qur'an and woman. Rereading the Sacred Text from a Woman's Perspective, New York \& Oxford. 
Erzählung die intersektionale Rahmung und den gegenderten antimuslimischen Rassismus. Während Hassan M. sich zunächst in der Weise der Anrufung unterwirft, dass er (zumindest) dieses Zeichen (»Arabisch lesen«) zu verbergen versucht, gehen beide in der Gruppendiskussion dazu in kritische Distanz. Hassan M. sagt: »Und dann aber natürlich hab ich mich irgendwann ( ) Also was für Schei:ße mache ich da? « Freilich können sie sich der Anrufung trotz reflexiver Distanzierung nicht entziehen. ${ }^{9}$ Sie sind weiterhin durch andere Zeichen markiert.

Für Fatouma K. hingegen spielt es keine Rolle, ob sie durch das Lesen bestimmter Bücher in einer spezifischen Schrift erkannt wird. Als Schwarze Hijabi entscheidet sie sich bewusst dazu, im öffentlichen Raum »demonstrativ« aufzutreten. Sie sagt: »Mir ist das egal. @ Ich lauf dann so, ich hab dann mein' James Baldwin oder meine muslimischen Sachen, ich (.) mach das demonstrativ« und fügt leise hinzu: »[U]nd also ${ }^{\circ}$ ich trag ja auch mein Kopftuch und so weiter ${ }^{\circ}$ demonstrativ. « Fatouma K. verknüpft die Anrufung zur Selbstführung mit intrinsischen Bedürfnissen, wenn sie das Kopftuchtragen einerseits als Demonstration bezeichnet und andererseits als religiöse Entscheidung:»[I]mmer noch ist es aus religiösen Gründen. "Sie teilt als Hijabi gegenderte Rassismuserfahrungen mit Leyla L., bezieht sich aber darüber hinaus intersektional auf antischwarzen Rassismus. Fatouma K.'s Erfahrungen kreuzen sich an der Schnittstelle von gegendertem antimuslimischem und antischwarzem Rassismus. Während Hassan M. »Arabisch lesen« und Leyla L. "Qur'an and Woman« als Anlässe für (antizipierte) gegenderte Rassismuserfahrungen identifizieren, führt Fatouma K. zunächst »James Baldwin«, den nicht-muslimischen Schwarzen Schriftsteller, und erst dann die "muslimischen Sachen« an, wobei sie beide als ihr zugehörig (»mein«, »meine«) markiert. Sie knüpft damit an die Debatte und die geteilte Erfahrung als >Muslim:innen $<$ an und grenzt sich gleichzeitig ab. Die doppelte Betonung des »demonstrativ[en]« Zeigens könnte auch diese Abgrenzung implizieren. Für Fatouma K. gehören ihr

9 Verschiedene Formen von Distanzierungen werden im Kapitel Selbst_Positionierungen interpretiert. 
Muslimisch- und ihr Schwarzsein ebenso untrennbar zusammen wie ihre antimuslimisch-antischwarze Rassismuserfahrung als Hijabi.

\section{»|ch kann meine Hautfarbe nicht ablegen«}

Fatouma K. ist die einzige Schwarze Hijabi in der dritten Gruppendiskussion. Sie macht wiederholt im Verlauf der Diskussion auf ihre spezifischen Erfahrungen von intersektional gegendert-antimuslimischantischwarzem Rassismus aufmerksam. Sie widerspricht in der folgenden Sequenz Asmah N. und Hassan M., die anhand verschiedener Beispiele problematisieren, welche weitreichenden Folgen es hat, wenn persönliche Fragen »voll unter dem Sicherheitsaspekt, volle Kanne«verhandelt werden mit dem Ziel, "mit weniger Problemen« zu leben. Asmah N. sagt: "Du betrachtest dich immer aus der Perspektive der anderen.« Die Selbstführung beginnt bei Überlegungen zur Familienplanung und Namensgebung, ragt in die Entscheidung hinein, in welcher Sprache im öffentlichen Raum gesprochen wird, bis hin zur Wahl von Kleidung, Schmuck und Make-up. Asmah N. resümiert, das ist »so ein intimer Eingriff « und Hassan M. meint, dass der Versuch »unsichtbarer $[\mathrm{zu}]$ werden« $\mathrm{zu}$ Überlegungen führt, die für die Person selbst »so falsch sind. Die anderen Diskussionsteilnehmer:innen stimmen wiederholt zu und darin überein, dass jene Marker, die zu ihrer Diskriminierung führen, $\mathrm{zu} » k$ kompensieren« versucht werden. Das setzt allerdings entsprechende Ressourcen voraus, man muss sie sich »leisten« können.

Als Asmah N. ihr Dilemma anspricht, wird sie von Fatouma K., die wie die anderen vorher mehrfach zugestimmt hat, unterbrochen. Fatouma K. sagt, dass sie Asmah N.s kostspielige »Kompensations-irgendwas « nicht mittragen kann, da auch diese Anlass zu Stereotypisierung bieten. Asmah N. kommentiert ihr eigenes Verhalten, indem sie Klasse ins Gespräch einführt: »Das kann ich mir natürlich leisten.« Fatouma K. bestätigt sie unterbrechend: »[W]ollte ich gerade sagen, sorry, aber ((lacht)) ich kann mir nicht « und fordert Asmah N. auf: »Mach's! « Die darauf folgende Irritation nutzt Fatouma K. und führt weiter aus: "Das sind hier halt die Entscheidungen, die Menschen treffen. Die ei- 
nen entscheiden sich so, die anderen so (.) und ob man sich davon tangieren lassen will, ich kann meine Hautfarbe nicht ablegen (2) Kann ich nicht (.).«Asmah N. nimmt den Faden zur eigenen Erzählung, in der sie sich entlang von Klasse distinguiert, nicht wieder auf, sondern knüpft an Fatouma K.s Abgrenzung entlang von Hautfarbe an. Sie fällt nun ihrerseits Fatouma K. ungeduldig zustimmend ins Wort (»Ja, ja, stimmt ja auch, stimmt ja auch«) und gibt anschließend zu bedenken: »[J]a, aber du kannst doch auch dein Kopftuch nicht ablegen.« Damit nimmt sie auf ihr situiertes Wissen Bezug, wonach es für viele Hijabis undenkbar ist, ihr Kopftuch abzulegen.

Die Rassifizierung von Muslim:innen knüpft unter anderem am Kopftuch an. Fatouma K. erwähnt das Kopftuch nicht, vielmehr bezieht sich ihr Einwand auf Kleidungsstile (»moderne Sachen«, »old school Kleidung«, »ethnic clothing«) und Schmuck (»Goldreifen«). Indem Asmah N. den Einwand von Fatouma K. auf diese Weise deutet, lässt sie Klasse als Differenz zwischen sich und Fatouma K. wieder fallen und appelliert an ihre geteilte Erfahrung als Musliminnen. Als Marker $\mathrm{zu}$ ihrer Rassifizierung funktionieren unter anderem das Kopftuch und die Hautfarbe. Fatouma K. kommt der Aufforderung nach, sich weiterhin als Muslimin zu subjektivieren: Sie lässt die Argumentation zur Hautfarbe jedoch fallen und antwortet lediglich auf den Einwand zum Kopftuch. Dennoch geht in ihre Analyse zur Funktionsweise von antimuslimischem Rassismus die Reflexion der Interrelation verschiedener Rassismen ein, die auf ihrem situierten Wissen gründet. So argumentiert sie, dass sie ihr Kopftuch zwar ablegen kann, dass das jedoch nichts an »bestimmte[n] Wissensbestände[n] in den Köpfen der Menschen« ändert. Als Schwarze Hijabi ruft sie zunächst die Unterscheidung zwischen antimuslimischem und antischwarzem Rassismus auf: Während aus ihrer Sicht das Kopftuch physisch abgelegt werden kann, ist die Hautfarbe biologisch nicht zu ändern.

Fatouma K.s Argumentation zielt indes nicht auf eine Unterscheidung, die Rassismus auf biologische >Rassen`konstruktionen reduziert. Ihr Widerspruch zu Asmah N.s Feststellung richtet sich auch nicht gegen die Existenz und die rassistische Aufladung snatürlicher< Unterschiede, hier der Hautfarbe. Vielmehr widerspricht sie der hegemonia- 
len Auffassung, wonach sich >Islamkritikı gegen die Praxis von Muslim:innen, hier das Tragen eines Kopftuchs, richtet und deswegen kein Rassismus sein kann. Rassismus ist keine Reaktion auf das, was rassifizierte Personen tun, sondern ein Ausdruck des Wissens, auf das Personen zurückgreifen, die sich rassistisch äußern. ${ }^{10}$ Aus Fatouma K.s analytischer Perspektive schützt ein auf Assimilation gerichtetes Entgegenkommen rassisierter Personen, hier das Ablegen des Hijab, nicht vor Rassismus. »[...] bestimmte Wissensbestände in den Köpfen der Menschen« wirken weiterhin und rassifizieren Muslim:innen auch ohne Kopftuch, wenn auch zum Teil auf andere Weise.

Auch Asmah N.s Aussage richtet sich sowohl gegen die Thematisierung von antimuslimischem Rassismus als >Islamkritik als auch gegen die unterkomplexe Problematisierung von Rassismus als >Hautfarbenrassismus`. Ihre Argumentation stützt sich aber weiterhin auf die Praxis von Muslim:innen und fordert die Anerkennung von Differenz im Verhältnis zur Gesamtgesellschaft ein. Sie setzt darauf, dass die Praxis von Muslim:innen verstanden und anerkannt wird, sodass diese frei entscheiden können, ob sie ein Kopftuch tragen oder nicht. An diesem Punkt unterscheiden sich die Argumentationen der beiden Diskutierenden: Während Fatouma K. das innermuslimische Verhältnis als diverses und intersektional diskriminiertes anspricht, argumentiert Asmah N. in Richtung des hegemonialen Diskurses. Auch Fatouma K. spricht in diese Richtung, allerdings nicht, um für Verständnis und Anerkennung zu werben, sondern um zur Selbstreflexion aufzufordern. Ihre Argumentation fokussiert nicht die Praxis von Muslim:innen: Nicht die Differenz ist hier das Problem, sondern die Differenzierung, die als Rassifizierung problematisiert wird. Ohne die Unterschiede zwischen den Rassismen $\mathrm{zu}$ vernachlässigen, verwischt Fatouma K. also die den antimuslimischen Rassismus als >Islamkritik« rechtfertigende Unterscheidung zwischen Hautfarbe und Kopftuch. Sie kann zwar das Kopftuch ablegen, wird aber weiterhin als Muslimin rassifiziert: Ob mit oder ohne Kopftuch, »bestimmte 
Wissensbestände« werden andere Marker finden, sodass der antimuslimische Rassismus, wenn auch anders, so doch auch dann greift, wenn eine Frau das Kopftuch ablegt. Die Praxis von Muslim:innen (Kopftuch tragen oder ablegen) ist nicht ausschlaggebend, sondern der Rassismus (»bestimmte Wissensbestände«). Fatouma K. erteilt dem Versuch, »unsichtbarer [zu] werden« als unmögliches Unterfangen eine Absage, weil Rassismus nicht als Reaktion auf die Praxis rassifizierter Personen zu verstehen ist. Die Reflexion ihrer eigenen Hypervisibilität trägt vor dem Hintergrund ihres situierten Wissens, das auf die reflektierte Erfahrung von gegendert-antimuslimisch-antischwarzem Rassismus zurückgreift, dazu bei, die in der Diskussion angeführten Unterwerfungspraktiken von vornherein zum Scheitern zu verurteilen und zu der widerständigen Schlussfolgerung zu gelangen: »Ich möchte mich nicht davon bestimmen lassen.«

\section{»Also (.) manchmal ist es wirklich gu::t, wenn ein anderer (.) für einen spricht."}

Aslı A. aus der ersten Gruppendiskussion gibt sich als säkular zu erkennen. Sie ist als türkische Frau markiert und obwohl sie nicht religiös ist, teilt sie die Erfahrung, als Muslimin adressiert zu werden. Auch sie ist von antimuslimischem Rassismus, wenn auch auf andere Weise als Hijabis oder praktizierende Muslim:innen, betroffen. Sie lebt wie Gülçin D. als Nachfahrin von türkischen Eingewanderten in Deutschland und versteht sich als Teil der Gesellschaft. Ihre antimuslimische Rassismuserfahrung lässt einen anderen Umgang mit dem Sicherheitsdiskurs zu, gerade weil sie sich als »säkular« positioniert und dennoch als >Muslimin entierungsrahmens zu Gülçin D. kann sie sich mit dem gemeinsamen "wir« im Raum identifizieren und wird von den anderen Diskussionsteilnehmer:innen zwar ambivalent, aber doch als säkulare Muslimin angesprochen. Aslı A. teilt die Erfahrung: »man wird automatisch als Bedrohung angesehen«. Gülçin D. kann ihr Schmunzeln jedoch nicht verbergen angesichts der Selbstrepräsentation nicht nur als säkulare, sondern darüberhinaus als nicht-religiöse Muslimin. Die Art und Wei- 
se, wie beide dieses Thema behandeln, zeigt, dass sich ihre Erfahrungswerte an diesem Punkt kreuzen und doch wieder auseinander laufen: Die Bedeutung der Adressierung und der Umgang damit und mit ihrem (vermeintlichen) Muslimischsein wird mehrstimmig geführt und enthält in dieser Sequenz Konfliktpotential.

Gülçin D. weist Aslı A. auf die Rolle ihres säkularen Verbandes hin, der zunächst im Sinne des Berliner Neutralitätsgesetzes argumentierte und deren Mitglieder als Kronzeug:innen für ein Kopftuchverbot gehört wurden. Aslı A. bedauert die ehemalige Position ihres Verbandes (»Das war vor meiner Zeit«), weil sie zum Schaden muslimischer Frauen führte, die Kopftuch tragen. Sie steht an der Seite von Hijabis, wie sie sagt, und wünscht sich mehr Aktivismus gegen das Verbot. Das Ziel, der institutionellen, intersektionalen Diskriminierung zu begegnen, trennt und verbindet beide: Aslı A. sagt, dass sie sich mehr »Gegenwind « von muslimischen Verbänden wünscht, um gegen »künstlich erzeugte Debatten « $\mathrm{zu}$ argumentieren. Sie spricht hier zwar ein religiös-markiertes Thema und die Verbände als religiöse an, meint aber nicht die Religion, sondern die Religiösisierung der »Debatten«, weswegen sie sie als »künstlich erzeugt« attribuiert. Aslı A. diskutiert >Religion « in einem anderen, nämlich säkularen Kontext. Sie vermisst einen "gesellschaftlichen Aufschrei seit dem Bekanntwerden der NSU-Verbrechen«, wenn ein »Zusammenhang zwischen Burkaträgerinnen und Terrorismus [...] erzeugt« oder »geflüchtete Muslime pauschal [als] Gruppenvergewaltiger« bezeichnet werden. Als Vorbild dient ihr die Aktion »Berlin trägt Kippa«. Sie verschiebt den Fokus, indem sie Widerstand gegen antimuslimischen Rassismus in jenen gegen andere Rassismen einbettet. Die Aufforderung der Vertreterin eines säkularen Verbands, gerichtet an die anwesenden Vertreter:innen religiöser Verbände, erscheint deswegen widersprüchlich, ist jedoch gemäß ihres eigenen Orientierungsrahmens stimmig: "Also die Kampagne äh Kontra Neutralitätsgesetz ist (.) äh (.) irgendwie (.) ä:hm muss belebt werden und da äh (.) zählen wir auch so n bisschen auf auf@eure Unterstützung@.«

Dieser Rahmung des Themas widerspricht Gülçin D. vehement. Sie weist Aslı A.'s Aufforderung zurück, dass sich Muslim:innen deutlicher in der Öffentlichkeit kritisch äußern sollen. Sie verengt den durch As- 
lı A. geöffneten Aktionsradius wieder und kehrt zum Ausgangspunkt der Diskussion zurück, dem Kopftuchverbot. Dieses schöpft aus einem hegemonialen Wissen über sie als praktizierende Muslimin, das ihrem eigenen Wissen über sich und ihre Praxis entgegensteht. Sie spricht aus eigener Erfahrung und perspektiviert das politische Thema in seiner persönlichen Bedeutung für sie als Hijabi. Das unterscheidet ihre Situation grundsätzlich von jener Aslı A.s. Gülçin D.s Argumentation zielt darauf, ihre muslimische Perspektive als direkt Betroffene sichtbar zu machen und zu stärken und gleichzeitig jene in die Verantwortung zu nehmen, die säkular-muslimisch sind und kein Kopftuch tragen. Aslı A. behandelt das »Neutralitätsgesetz«als Thema, das in einem Zusammenhang mit anderen Themen steht, die der politischen und zivilgesellschaftlichen Positionierung und Aktion bedürfen. Für Gülçin D. dagegen stellt das Kopftuchverbot kein abstraktes Thema dar, vielmehr betrifft sie das Verbot direkt und hat konkrete Konsequenzen für ihr eigenes Leben. Obwohl säkular und religiös in der Regel keine Gegensätze sind, bezieht sich Gülçin D. aufgrund der sichtbar gelebten religiösen Praxis auf einen anderen Orientierungsrahmen als Asl A. Beide deuten das Verbot vor dem Hintergrund ihrer jeweiligen, von jener der anderen an diesem Punkt sich unterscheidenden Erfahrungen und Positionierungen. Beide fordern Unterstützung von den jeweils anderen ein, die Begründung und die Adressierung fällt aber entgegengesetzt aus: Während Aslı A. die muslimischen Verbände auffordert, bei »Kampagnen« mitzumachen, um sie zu »beleben«, wünscht sich Gülçin D. als direkt vom Verbot betroffene Hijabi solidarische Unterstützung. Mehr noch, sie sagt: »Also (.) manchmal ist es wirklich gu::t, wenn ein anderer (.) für einen spricht.«

Als Hijabi hat Gülçin D. »auf ganz vielen Diskussionsforen« gesprochen, aber: »[W]enn ich mich eben gegen das Neutralitätsgesetz als Musli::min ausspreche, dann kommt das bei den Leuten ga:nz anders an, als, wenn da eine: (.) äh Sabine sitzt, die (.) eben im Sinne der Neutralität das sagt."Wenn sie sich für ihr Recht einsetzt, das Kopftuch zu tragen, wird das dahingehend interpretiert, dass Muslim:innen »Druck« ausüben, womit das Bedrohungsszenario bestätigt wird: "Also es sind die radikalen Muslime: die wollen eben, dass alle Frau- 
en ein Kopftuch tragen und seht ihr, sie sind auch gegen die Neutralität unseres Staates (.) und da fängt ja schon die Islamisierung an und so. Gülçin D.s Einsatz für ihr Recht, Kopftuch zu tragen, wird im Sicherheitsdiskurs als Gefahr für die Gesellschaft umgedeutet. Einerseits werden Muslim:innen ununterbrochen genötigt, zu gewissen Themen Stellung zu beziehen. Andererseits werden sie nicht gehört und ihre Antworten antimuslimisch umgedeutet. Einen Ausweg aus diesem Dilemma sieht Gülçin D. darin, dass Verbände für Muslim:innen sprechen, die nicht direkt betroffen sind, aber mit denen es partielle Übereinstimmungen gibt, nämlich einerseits säkular-migrantische und andererseits christliche Verbände. Diesen Einwand hört Aslı A. nicht, da ihr Orientierungsrahmen ein anderer ist.

Beide sprechen aus der Erfahrung einer rassifizierten muslimischen Frau, die eine trägt Kopftuch, die andere nicht. Insofern unterscheiden sich ihre Rassismuserfahrungen an diesem Punkt. Gülçin D.s Intervention zielt darauf, die Perspektive von Hijabis zu stärken und gleichzeitig vor dem Hintergrund ihrer Erfahrung die Aufforderung zurückzuweisen, sich als Muslim:innen in dieser Frage mehr zu engagieren. Vor dem Hintergrund ihres situierten Wissens als Hijabi ist ihr Engagement gegen das Kopftuchverbot kontraproduktiv. Indem Gülçin D. nur an dem Argument von Aslı A. anknüpft, das für sie als Hijabi relevant ist, verschiebt sie die Aufforderung zu allgemeinem gesellschaftlichem Engagement hin zu einer eindringlichen Bitte um Unterstützung in einer Sache, in der sie als betroffene Person nicht am Diskurs partizipieren kann, weil sie nicht gehört wird. Vor Gülçin D.s Erfahrungshintergrund ist die Verschiebung nachvollziehbar, weil sie ihre Situation als Dilemma erlebt: Musliminnen sehen sich als direkt und gravierend von antimuslimischem Rassismus Betroffene permanent gezwungen, für ihre Rechte zu kämpfen und Stellung zu beziehen. Auf ihre Argumente wird aber nicht eingegangen: Weder ihre wiederholten Erklärungen, dass sie aus anderen als den vermuteten Gründen Kopftuch tragen, noch, dass sie selbst entscheiden wollen, ob sie es tragen oder nicht, erhalten Resonanz. Gülçin D. erlebt nun in der Gruppendiskussion wieder, dass ihre Interessen ins Leere laufen. Sie selbst geht ebenfalls nicht auf Asl A.s Argumentation ein, sodass 
die persönliche Not der einen dem politischen Anliegen der anderen gegenübersteht. Beide knüpfen zwar aneinander an und bestätigen sich gegenseitig in der Notwendigkeit, das Ziel zu erreichen (das Kopftuchverbot abzuschaffen), reden aber letztlich doch aneinander vorbei, indem sie nicht auf die Argumente der jeweils anderen eingehen, sondern ihre eigene Strategie verfolgen.

Viola E. versucht sich wiederholt in die Diskussion einzubringen, die von langen Redebeiträgen geprägt ist. Schließlich unterbricht sie und sagt:

»Ich muss mal ganz kurz was, auch wenn=es vielleicht nicht direkt dazu gehört, was sagen. Weil es immer heißt gegen Neutralitätsgesetz. Ich finde es fängt schon bei den Begriffen an. Ähm das klingt so, wie man ist gegen Neutralität. Natürlich sind wir ja für Neutralität. Aber, hier gehts nicht um Neutralität, sondern hier gehts um Berufsverbote."

Aslı A. unterbricht zweimal: »von Frauen« und »Berufsverbote, von denen Frau=Frauen betroffen sind «, beim zweiten Mal fällt ihr wiederum Gülçin D. ins Wort: "Hauptsächlich muslimische Frauen«, woraufhin Viola E. sagt: "Genau. Diskriminierung von Frauen.« Alle drei betonen also, dass das Berufsverbot nur Frauen diskriminiert. Während es Gülçin D. wichtig ist, zu betonen, dass es sich »[h]auptsächlich [um] muslimische Frauen« handelt, belassen es Aslı A. und Viola E. bei der gegenderten Problematisierung.

Die unterschiedlichen Deutungen der drei an dieser Sequenz beteiligten Diskussionsteilnehmerinnen stehen im Zusammenhang mit ihren aktuellen Erfahrungen mit dem Kopftuchdiskurs. Weder Aslı A. noch Viola E. teilen die Erfahrung von Gülçin D., nämlich als Hijabi diskriminiert, missgedeutet und ausgeschlossen zu werden. Indem auch Viola E. Gülçin D. korrigiert und ihr sagt, welche Begriffe angemessen sind und verwendet werden sollen, bestätigt sie Gülçin D.s These, wonach Musliminnen mit Hijab nicht gehört werden. Für sie als Kopftuch tragende Person stellt das Gesetz ein Kopftuchverbot dar. Gülçin D. fordert sowohl eine sprachliche Konkretisierung des Verbots, die Hijabis als Betroffene im Blick behält, als auch eine effektive Unterstützung 
zur Erlangung ihrer Rechte ein. In diese Diskursivierung sind Aslı A. und Viola E. nicht als direkt beteiligte Personen eingeschlossen, vielmehr fordert eine direkt Betroffene von ihnen solidarisches Handeln ein. In dieser Sequenz bleibt diese allerdings aus, sodass Gülçin D.s Ohnmachtsgefühl, das aus den "ganz vielen Diskussionsforen « resultiert, aktualisiert und bestätigt wird. Sie wird selbst von jenen nicht gehört, die das gleiche Ziel verfolgen wie sie. Ihrer Erfahrung nach ist es manchmal »besser [...], wenn nicht die muslimischen Verbände (.) sich [zu bestimmten Themen] aussprechen «, sondern sich zurückhalten und anderen das Feld überlassen, die für ihr Anliegen, das sie direkt betrifft, eintreten. Trotz gemeinsamen Ziels ist aus jeder der drei Perspektiven die Diskussion zum Kopftuch-/Berufsverbot indes gescheitert.

\section{"guck mal du bist aus einer anderen Generation "}

In der ersten Gruppendiskussion fließen Migrationserfahrung sowie Alter und Generation in die Reflexion des erlebten antimuslimischen Rassismus ein. In der bereits erwähnten und im Zusammenhang mit gegendertem antimuslimischem Rassismus diskutierten Sequenz beschreibt Rahman F., dass er als Vorstand einer Gemeinde nachbarschaftlichen Konflikten durch Dialog begegnen möchte. Er plädiert dafür, Bürger:innen die medial geschürte Angst vor Muslim:innen zu nehmen, auf sie zuzugehen und sie für sich zu gewinnen. In seinen Ausführungen erscheinen in der Gegenüberstellung zur »Zielgruppe [...] Bü:::rger« Muslim:innen nicht als Bürger:innen, sondern als Eingewanderte. Das korrespondiert mit seiner persönlichen Positionierung; ob sie auch jene seiner Gemeinde spiegelt, wissen wir nicht.

Gülçin D. überzeugt der Vorschlag zum Dialog nicht. Ihr zufolge machen die Gemeinden das schon seit Jahren mit wenig Erfolg. Gülçin D. betont, dass sie ihre Zugehörigkeit zur Gesellschaft nicht beweisen muss: Sie ist hier geboren und verdient die gleichen Rechte wie alle anderen auch. Der Beweispflicht setzt sie die Forderung entgegen, als Teil der Gesellschaft anerkannt zu werden. Sie begründet ihre Rechte hier mit einem Argument, das die Lebenssituation der sersten Generation nicht mit einbezieht, sodass diese in der so begründeten Forde- 
rung nach Anerkennung unberücksichtigt bleiben. Gülçin D.s und Rahman F.s Erwartungen an die Gesamtgesellschaft und ihr Umgang damit knüpfen an ihren unmittelbaren eigenen Erfahrungen an. Gülçin D. möchte sich nicht (mehr) von bestimmten Bildern über Muslim:innen distanzieren oder Nicht-Muslim:innen aufklären, sondern sich als gleichberechtigte Muslimin dann äußern, wenn es ihr angemessen erscheint. Sie nimmt Bezug auf die Unterscheidung von Eingewanderten nach Generationen: Im Gegensatz zu Rahman F. (erste Generation) ist sie hier geboren (x'te Generation). Sie erklärt ihm: »[G]uck mal $\mathrm{du}$ bist aus einer anderen Generation. Du bist aus der Generation, die als (1) GASTarbeiter gekommen ist.« Gülçin D. zählt Rahman F. zu den älteren >Gastarbeiter:innen`. Gemäß ihrer eigenen Familiengeschichte korrespondieren Alter und Einwanderungsgeneration mit politischem und persönlichem Migrationskontext. Gülçin D. unterscheidet die Deutungen zu und Umgangsweisen mit dem antimuslimischen Rassismus vor dem Hintergrund der jeweiligen Zugehörigkeit zur Gesellschaft: »hier geboren« versus "gekommen«. Die Differenz zwischen sich und ihren (Groß-)Eltern dient ihr dabei sowohl als Muster, das sie auf die Adressierung von Rahman F. anwendet, als auch als Erfahrungsdifferenz, die sie - aufgrund ihrer Zugehörigkeit zu einer anderen Migrationsgeneration - als die Erfahrenere (»guck mal«) von ihnen beiden im Umgang mit der Migrationssituation auszeichnet. Als »hier geboren[e]« hebt sich Gülçin D. im Verhältnis zu Rahman F. mehrfach (Gender, Position, Alter, Generation) hervor: »Herr Sheikh abi [...] guck mal«.

Rahman F. unterbricht Gülçin D.: Er ist als Student nach Deutschland gekommen (»nein, nein, ich bin Student als=als Student«), ihn als 'Gastarbeiter anzusprechen ist demnach inkorrekt. Der intersektionalen Selbst- und Fremd-Positionierung fügt Rahman F. durch Klasse ein weiteres Differenzverhältnis zwischen den Diskussionsteilnehmer:innen hinzu. Im Unterschied zur Thematisierungsweise der anderen Differenzverhältnisse ist diese jedoch im bundesdeutschen Islamdiskurs recht jung und trifft ins Zentrum des Sicherheitsdiskurses. Die Markierung sarabischer Student< unterscheidet sich von jener >türkischer Arbeitsmigrant<, wird aber in der Diskussion nicht aufgegriffen; Rahman F.s Einwurf bleibt insgesamt ohne Resonanz. Interessanterweise wirft 
er weder seinen Beruf noch seine Position in der Gemeinde in die Waagschale, auch Gender und Alter nutzt er hier nicht, um seiner Zurücksetzung ("guck mal«) zu begegnen. Gülçin D. lässt sich nicht bremsen und führt ihre Argumentation fort, wie weiter oben bereits ausgeführt. Rahman F. kann erst an der Diskussion partizipieren, als er den Faden wieder aufgreift und seine Überlegungen zum eingangs erwähnten Nachbarschaftskonflikt weiterführt. Im Austausch über die Probleme, die sich ihnen als Muslim:innen stellen, können er und Gülçin D. wieder mit einem gemeinsamen Ziel vor Augen diskutieren. Ihre unterschiedlichen Positionen werden hier zwar auf unterschiedliche Positionierungen im intersektionalen Gefüge des antimuslimischen Rassismus zurückgeführt, flankieren aber nur die Diskussion und führen letztlich nicht dazu, entlang von Intersektionen Differenzen festzuschreiben.

\section{»Manchmal ist es ein Privileg sogar nicht hier geboren zu sein."}

Die unterschiedlichen Deutungen von Menschen, die erst als Erwachsene nach ihrer Migration Rassismuserfahrungen machen, und jenen, die bereits damit großgeworden sind, kommen auch in der dritten Gruppendiskussion zur Sprache. Hassan M., der einzige nicht in Deutschland sozialisierte Teilnehmer in dieser Gruppendiskussion, beschreibt, dass er an seinem eigenen Verhalten im öffentlichen Raum einen Unterschied bemerkt zwischen seiner Kindheit und Jugend in einem arabisch-sprachigen Land und seinem Leben als Student in Deutschland heute. Er fühlt sich unwohl ${ }^{11}$, wenn er öffentlich ein arabisches Buch liest. Hassan M. reflektiert, dass der antimuslimische Rassismus dazu führt, dass er sich zensiert und bestimmte Orte und Situationen meidet, um sich zu schützen. Er unterwirft sich also dem Diskurs, aber nicht, indem er sich assimiliert, sondern indem er seine Lektüre und seine Mobilität einschränkt. Die anderen Diskussionsteilnehmer:innen knüpfen mit eigenen Erzählungen an seine Schilderung an. Im Unterschied zu den anderen führt er jedoch die Differenz zwischen seinem

11 Zur emotionalen Dimension von Rassismuserfahrungen anhand dieser Sequenz: Keskinkılıç 2021 i.E. 
Verhalten vor und nach der Migration an. Er hat heute in manchen Situationen das irritierende Gefühl, nicht mehr er selbst sein zu können. Sein Erfahrungswissen aus einem anderen gesellschaftlichen Kontext ist ihm noch präsent. Leyla L. dagegen ist mit dem antimuslimischen Rassismus aufgewachsen. Sie verbindet das Lesen in der Öffentlichkeit mehr mit dem Gefühl der Scham und der Angst - Gefühle, die ihr Verhältnis zur deutschen Gesellschaft ausdrücken. Das wiederum überrascht Hassan M.: »echt? « In einer anderen Sequenz gesteht er, dass er erleichtert ist, nicht in Deutschland aufgewachsen zu sein: »Manchmal ist es ein Privileg sogar nicht hier geboren zu sein.«

Leyla L. spricht darüber, was die alltägliche Erfahrung von Rassismus mit Menschen macht, die davon unmittelbar betroffen sind. Einerseits freut sie sich, dass sie selbst und viele andere es schaffen, sich davon nicht allzu sehr beeinträchtigen zu lassen: »Krass ja, ma'sha'alla, was wir so alles erleben so und was wir so durch den Tag tragen (.) und krass- el'hamd'ul'illah, dass ich so heil- dass ich noch so glücklich bin so.« Ihre Freude gründet jedoch nicht auf einer Verharmlosung der Dinge, die »wir so durch den Tag tragen«. Sie betont: »[D]iese

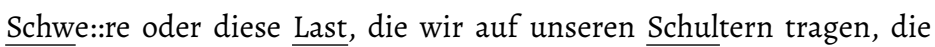
ist doch nicht norma::1.« Angesichts dessen und »im Umkehrschluss« ist sie dann doch "auch ein bisschen stolz«, dass sie nicht zerbricht und sogar "glücklich« sein kann. Diese Möglichkeit führt sie darauf zurück, vergessen und verdrängen zu können: »[I]ch frag mich natürlich, ob ich das- (.) ob das einfach (.) passiert, dass ich es vergesse oder ob das vielleicht auch irgendwi:e m: ob es 'n unbewusster Prozess ist, um es immer wieder zu verdrängen." Einerseits ist es hilfreich, vergessen und verdrängen zu können, denn so kann sie »heil« bleiben und »glücklich« sein. Andererseits ist ihr das nicht geheuer, sie sieht »natürlich«, dass "voll viele Menschen aus unserer Community« psychisch darunter leiden, "kaputt « gehen und »mit so Sachen nicht umgehen können«. Sie resümiert: »[E]s zieht nicht an uns vorbei.« Fatouma K. bestätigt die "psychische Dimension« von Rassismuserfahrungen: »[E]s gibt halt auch Leute, die diesem Druck nicht standhalten können und (.) und die nicht eine Überlebensstrategie finden (.) und dann in der Resigna- 
tion verharren, vielleicht es auch schaffen wieder rauszukommen und es immer so ein Pingpong-Ding ist.«

Hieran knüpft Hassan M. an. Er leitet seine Ausführungen mit der Frage ein, ob seine Vermutung zutrifft, dass er als einziger nicht in Deutschland geboren und aufgewachsen ist. Das bis zu diesem Zeitpunkt eine Stunde währende Gespräch und insbesondere die letzten Ausführungen haben ihn zu dieser Schlussfolgerung verleitet, die von den anderen bestätigt wird. Die zunehmend persönlicher und reflexiver ausfallenden Redebeiträge verdeutlichen die sich ständig neu formierenden Konstellationen von Gemeinsamkeiten und Unterschieden zwischen den Beteiligten. Hier ist es nun die Frage nach den Möglichkeiten der emotionalen und psychischen Verarbeitung von Rassismuserfahrungen vor dem Hintergrund des Nicht_Aufwachsens in einer rassistisch strukturierten Normalität.

Hassan M. kann auf ein anderes Selbstbild zurückgreifen als die anderen Diskussionsteilnehmer:innen. Es beruht auf seiner biographischen Erfahrung, einer Gesellschaft, hier seines Geburtslandes, selbstverständlich anzugehören. Rassismuserfahrungen be_treffen ihn nun auch, aber berühren ihn auf andere Weise: Sie sind ihm präsent und irritieren sein Selbstkonzept. Die Herausforderung, sich selbst permanent »aus der Perspektive der anderen« $\mathrm{zu}$ evaluieren, ist ihm neu. Die Scham, von der Leyla L. im Zusammenhang mit der öffentlichen Lektüre spricht, das Vergessen und Verdrängen der "schweren Last«, auch Fatouma K.s Ausführungen zu Überlebensstrategien und der Resignation thematisieren ihr Verhältnis zur weißen Gesellschaft. Auch ihr Selbstverhältnis ist daran orientiert, sie sprechen als (marginalisierte) Deutsche. Hassan M. greift auf einen anderen Erfahrungshintergrund zurück. Im Unterschied zu den hier Aufgewachsenen erinnert Hassan M.s Irritation ihn daran, dass er mit einer Situation konfrontiert ist, die ihn aus der Gesellschaft heraushalten will: »Am Ende sozusagen ich bin Outsider (.), ich gehöre nicht dazu.«

Er sagt »Outsider« und nicht »Ausländer«, denn selbst der deutsche Pass, wie die Diskussionsteilnehmer:innen später feststellen, ändert nur graduell etwas an ihrem Selbstverhältnis angesichts von Rassismuserfahrungen. Seine Erfahrung von Nicht-Zugehörigkeit gepaart 
mit der Erinnerung an eine zwar zurückliegende, aber dennoch seinerzeit selbstverständliche Zugehörigkeit lässt ihn mit anderen Gefühlen zurück. Er kann sich an seiner Erinnerung orientieren und vor diesem Hintergrund die aktuelle Situation als neue Herausforderung reflektieren. Sie erschüttert weder sein Selbstbild grundlegend noch lässt sie ihn (zu diesem Zeitpunkt) daran zweifeln, den aktuellen Zustand überwinden zu können. Die Erfahrung von Nicht-Zugehörigkeit der hier Sozialisierten gründet dagegen auf einem Widerspruch zwischen ihrem Gefühl von Zugehörigkeit und ihrer Adressierung als fremd. Ihre Reflexion nimmt die "Story of our Life« als ambivalent in den Blick. Hassan M. ahnt, dass diese Positionierung ihm ebenfalls bevorsteht und wird darin von Asmah N. bestätigt. Niemand in der Gruppe kommt auf die Idee, dass die unterschiedlichen Gefühle und Deutungen mit individuellen oder kulturellen Eigenschaften zu tun haben könnten, wie dies in hegemonialen Kontexten rassifizierend üblich ist.

In der weiter oben geschilderten Perspektivierung ergänzen Hassan M. und Asmah N. sich in der Aufzählung von Situationen, in denen sie sich angesichts des Sicherheitsdiskurses selbst führen. Hassan M. berichtet von seinen Versuchen, »unsichtbarer [zu] werden« und dass sich das »so falsch« anfühlt. Die Spannung bezieht sich auf sein altes im Vergleich zu seinem neuen Leben, die Selbstführung ist (noch) nicht internalisiert und zu seiner Normalität geworden. Er kann (noch) unterscheiden zwischen einem Verhalten, das sich für ihn selbst richtig anfühlt im Unterschied zu einem, das ihn mit einem Fremdbild konfrontiert, dessen mögliche Affirmation er reflektieren und dessen konkreten Gefahren aus dem Weg gehen muss. Der sicherheitspolitisch aufgeladene, rassistische, gesellschaftliche Kontext versetzt ihn in Unsicherheit im doppelten Sinne: Er verunsichert ihn emotional angesichts des Abgrunds zwischen Selbst- und Fremdbild und er gefährdet seine bürgerliche Unbescholtenheit, denn ein arabisch lesender junger Mann passt in das Profil eines >Gefährders $<.{ }^{12}$ Die hier sozialisierten Diskussionsteilnehmer:innen orientieren ihre Reflexion dagegen ausschließlich an der Differenz zwischen Selbst- und Fremdbild, die ihnen ein Leben 
lang bekannt ist und als solche unterschiedliche Kontextualisierungen kennt, jedoch keine selbstverständliche Zugehörigkeit zu jener Gesellschaft, in der sie ihren Lebensmittelpunkt haben. Sie kommentieren den Unterschied zwischen jenen, die anderswo aufgewachsen sind und auf andere Selbstbilder zurückgreifen können und ihnen selbst, die in einer weißen Dominanzgesellschaft, die sie als fremd diskursiviert, groß geworden sind. Sie können Hassan M.s Situation nachvollziehen, wenn sie sie mit eigenen Reiseerfahrungen im Rahmen eines Auslandsstudiums etwa vergleichen oder die Erfahrungen von Familienangehörigen und Freund:innen zitieren. Tasnim J. kommentiert: »Ganz ehrlich, irgendwie bin ich schon ein bisschen neidisch so.«

Hassan M. ist selbst eingewandert, gehört aber einer anderen Altersgruppe an als Rahman F. aus der ersten Gruppendiskussion, der ebenfalls zu Studienzwecken einreiste. Zwischen ihren Migrationen liegen indes Jahrzehnte. Beide gehören zwar vor dem Hintergrund der Kategorisierung der deutschen Migrationsforschung biographisch der gleichen >Migrationsgeneration an, aber unterschiedlichen Altersgenerationen. Auch Mohamed B. aus der ersten Gruppendiskussion ist im gleichen Alter wie Hassan M. und vor nicht allzu langer Zeit als Jugendlicher nach Deutschland eingewandert. Im Disput zwischen Gülçin D. und Rahman F. stimmt Mohamed B. teils Gülçin D. zu, die seinem Alter näher ist, andernteils Rahman F., der wie er auf eine eigene Migrationserfahrung zurückblickt. Auch Mohamed B. fordert Gleichberechtigung als hier lebender Bürger und weist die Verantwortung für die >Ängsteく der weißen Gesellschaft zurück. In der dritten Gruppendiskussion besteht ein Konsens darüber, dass rassifizierte Personen nicht verantwortlich für Rassismus sind, hier unterwirft sich auch Hassan M. nicht dieser Anrufung. Unterwerfung und Widerstand angesichts der Adressierung als bedrohlich fremd wird in den Diskussionen also vor dem Hintergrund vielschichtiger und sich in konkreten Situationen immer wieder neu intersektional kreuzender Positionierungen und Selbstidentifizierungen unterschiedlich verhandelt. Die Komplexität des antimuslimischen Rassismus und der damit verwobenen politischen, gesellschaftlichen, biographischen und persönlichen Dimensionen sowie die Vielfalt an damit einhergehenden 
Konstellationen hat changierende Positionierungen auch aufgrund des Zusammenspiels verschiedener Macht- und Differenzverhältnisse zur Folge, die wiederum zu lebendigen Gesprächsdynamiken und unterschiedlichen Allianzen und Strategien führen.

\section{Ent_Solidarisierung}

In allen Gruppendiskussionen wird auf das gesellschaftliche Misstrauen gegenüber $>$ Muslim:innen Bezug genommen. Der Sicherheitsdiskurs produziert ein Klima der Verdächtigung und Grenzziehung, das auch das Verhältnis innerhalb der Community berührt. (Als) Muslim:innen (Markierte) werden implizit oder explizit aufgefordert, sich $\mathrm{zu}>$ muslimischen (Gewalttaten bzw. zu Aussagen und Handlungen anderer (als) Muslim:innen (Markierter) $\mathrm{zu}$ positionieren. Zudem sehen sie sich in alltäglichen, beruflichen und zivilgesellschaftlichen Zusammenhängen dazu angehalten, Kontakte jeglicher Art daraufhin $\mathrm{zu}$ überprüfen, ob diese Personen oder Gruppierungen berühren, die unter Beobachtung stehen, um nicht selbst unter Verdacht zu geraten. Insbesondere der Verfassungsschutzbericht übt als sicherheitspolitisches Instrument nicht nur direkt, sondern auch indirekt Einfluss auf die muslimische Bevölkerung aus. Organisationen, die dort erwähnt werden, erfahren einschneidende Sanktionen, und zwar auch dann, wenn sie lediglich unter Beobachtung stehen. Nicht im Verfassungsschutzbericht erwähnt $\mathrm{zu}$ werden, bleibt trotzdem nicht folgenlos: Zum einen kann sich dies jederzeit ändern, zum anderen besteht die Gefahr, wegen der sogenannten Kontaktschuld Restriktionen zu erfahren. Insofern operiert der Verfassungsschutz an der Schnittstelle von Disziplinarmacht und Gouvernementalität. Im Kontext von Selbstführung gibt er ein eindrückliches Beispiel dafür, wie (als) Muslim:innen (Markierte) die Perspektive des hegemonialen Sicherheitsdiskurses einnehmen und sich in seinem Sinne selbst führen. Zwang und Strafe greifen (noch) nicht, sondern die institutionellen und strukturellen Rahmen von Beobachtung und Misstrauen führen dazu, dass sich (als) Muslim:innen (Markierte) selbst und gegenseitig disziplinieren und 
kontrollieren, indem sie ihr Verhalten und ihre Zusammenarbeit am Sicherheitsdiskurs ausrichten. Die Vorwegnahme der Sanktion führt dazu, dass die Sanktion nicht mehr nötig wird, weil sie schon im Vorfeld greift.

Dennoch sind die Diskussionsteilnehmer:innen dem Sicherheitsdiskurs nicht ausgeliefert. Unterwerfung ist nicht die einzige und vorrangige Praxis: Sie ringen um Differenzierung, handeln aus, mit wem es möglich ist, solidarisch zu sein und mit wem nicht, ohne sich selbst und die eigene Gemeinde allzu sehr in Gefahr zu bringen. Zum einen gibt es eine chronologische Abfolge, der zufolge Solidarität auf Entsolidarisierung folgt. Zum anderen analysieren die Diskussionsteilnehmer:innen, warum sich überhaupt die Frage nach Ent_Solidarisierung stellt, wenn >wir $<$ ohnehin divers sind und alle >Muslim:innen $<$ potentiell in Verdacht geraten und unter Beobachtung stehen. Tatsächlich deutet sich in der Dynamik der Gespräche selbst eine gelebte Praxis der Solidarisierung und des Widerstands gegen allzu große Vereinnahmung ab. Diese ist weder harmonisch noch zielt sie auf Übereinstimmung, sondern schöpft aus der gemeinsamen Erfahrung, als Bedrohung adressiert zu werden und auch aus der Unklarheit der Kriterien. Da lediglich die (vermutete) Religionszugehörigkeit zum Islam als Kriterium sicher ist, vermag es die essentialistische und homogenisierende Ansprache, $>$ Muslim:innen< trotz (teils erheblicher) Differenzen an diesem Punkt zu vereinen, sodass sie füreinander einstehen.

Die Solidarisierung äußert sich in den Reflexionen der Beteiligten. Sie greift auf die Erfahrungen anderer `Muslim:innen zurück und wird durch eigene Perspektiven ergänzt. Die Diskussionsteilnehmer:innen tauschen sich über die Erfahrung mit dem Sicherheitsdiskurs aus und erschaffen damit einen Raum, in dem das Problem intern verhandelt und aus verschiedenen Blickwinkeln diskutiert wird. Deutlich wird, dass die Frage nach einer möglichen Ent_Solidarisierung weniger vor dem Hintergrund der eigenen Einschätzung zur politischen, gesellschaftlichen oder religiösen Ausrichtung eines potentiellen Kooperationspartners diskutiert wird als auf Basis der Vorsicht vor einem Kontaktschuldvorwurf. Die Notwendigkeit, sich selbst und die eigene Organisation zu schützen, durchkreuzt die Solidarität als Community, 
der Gemeinschaft als Muslim:innen, in der füreinander eingestanden und solidarische Werte miteinander gelebt werden. Dabei treten ambivalente Positionierungen und Handlungsspielräume mit Blick auf gemeinsame Visionen für eine muslimische Agency an den Tag.

In den Gruppendiskussionen werden Risiken, Chancen und Herausforderungen (öffentlich vernehmbarer) Solidaritätsbekundungen mit anderen Muslim:innen kritisch reflektiert. Insgesamt scheint für die Diskussionsteilnehmer:innen solidarisches Handeln und manchmal sogar eine Solidaritätsgeste kein leichtes Unterfangen $\mathrm{zu}$ sein, könnten sie doch im öffentlichen Raum missgedeutet werden oder den sich Solidarisierenden und ihrem Anliegen schaden. Die allgemeine Stimmung von Bedrohung, Verdacht und Unsicherheit dringt in den Diskussionsraum ein und fordert die Sprechenden dazu auf, sich zu positionieren und das eigene Handeln angesichts des Bedrohungsszenarios zu legitimieren. Die Erfahrung, für das Verhalten anderer >Muslim:innen zur Rechenschaft gezogen $\mathrm{zu}$ werden, wird jeweils vor dem Hintergrund eigener Erfahrungen perspektiviert, kommentiert und bewertet. Insofern hängt die Ent_Solidarisierung zwischen verschiedenen muslimischen Gruppierungen mit ihrem Antwortverhalten auf ihre hegemoniale Adressierung und der ihnen zugewiesenen bzw. verwehrten Verantwortungsübernahme gegenüber der Gesamtgesellschaft zusammen ${ }^{13}$. Die Unterwerfung unter das Eine kann als Widerstand gegen das Andere gedeutet werden und entsprechende Effekte zeitigen. Das Changieren zwischen Ver_Antworten und Ent_Solidarisierung bringt jeweils neue Reflexionen und Entscheidungen hervor, die im konkreten Fall neu verhandelt werden, sodass jedenfalls in unserem Sample - solidarisches Verhalten untereinander bzw. Unterwerfung unter den Sicherheitsdiskurs nicht bestimmten Personen oder Gruppierungen zugeordnet werden können: Beides wird gleichzeitig und in Relation zueinander konkret und kontrovers diskutiert, und häufig kommt es nicht zu rigorosen Positionen und eindeutigen Entscheidungen, sondern der Austausch über die verschiedenen Dimensionen und das Abwägen möglicher Folgen steht 
im Mittelpunkt. In den Diskussionen zeigt sich auch, wie ermüdend und letztlich unbefriedigend die Verhandlung von Ent_Solidarisierung angesichts drohender Disziplinarmaßnahmen ist und welche Auswege daraus gesucht werden.

\section{„Verhaltens- (.) äh ra- Verfassungs- ä:h schutzbericht ist sozusagen unsere Verhaltensgrundlage oder unsere Aktionsgrundlage"}

Abdul I. ist Vertreter eines muslimischen Verbandes und nimmt an der zweiten Gruppendiskussion mit zwei weiteren Vertreter:innen anderer muslimischer Verbände teil, die ebenfalls in der Vergangenheit direkt von Praktiken der Sicherheitsbehörden betroffen waren. Er gibt zu bedenken, dass die Zusammenarbeit unter Muslim:innen angesichts zunehmender Verdächtigungen erschwert wird:

»Es gibt die Tendenz (.) ä:hm durch diesen Druck, der entsteht das machen dann Organisationen auch, ne, also indem sie dann einfach sagen ok Verhaltens- (.) äh ra- Verfassungs- ä:h schutzbericht ist sozusagen unsere Verhaltensgrundlage oder unsere Aktionsgrundlage, was ja klar ist wegen den Förderungsrichtlinien und so weiter.«

Der Verfassungsschutzbericht wirkt aus dieser Perspektive in mehrere Richtungen. Er ist ein Instrument des Staates, um unter Verdacht gestellte Gruppierungen zu beobachten. Diese werden nicht erst bei erwiesenem Fehlverhalten sanktioniert, sondern bereits die Erwähnung im Verfassungsschutzbericht zieht Sanktionen nach sich, die von der Beobachtung, über das Versagen öffentlicher Gelder bis hin zum Verlust der bürgerlichen Reputation reichen. Der Verfassungsschutzbericht wirkt jedoch auch nach innen hin. Zum einen in Gestalt des Unter-Beobachtung-Stehens der dort aufgeführten Gruppierungen, zum anderen wirkt das (Noch-)Nicht-unter-Beobachtung-Stehen bereits im Vorfeld: Mit einer der im Verfassungsschutzbericht genannten Personen oder Gruppierungen in Verbindung gebracht $\mathrm{zu}$ werden, kann dazu führen, selbst im nächsten Verfassungsschutzbericht aufgeführt zu werden, also der >Kontaktschuld < bezichtigt zu werden. Das 
bedeutet, dass staatliche Sanktionen drohen, sobald die Selbstführung versagt. Die Disziplinarmacht kann auch in der Gouvernementalität greifen, Zwangsmaßnahmen und Selbstführung bleiben in enger Verbindung bestehen.

Das hat zur Folge, dass es muslimische Gruppierungen gibt, die im Verfassungsschutzbericht nicht erwähnt sind und nicht beobachtet werden, und die dennoch aus Angst, der >Kontaktschuld verdächtigt und sanktioniert zu werden, andere Gruppierungen und Personen kritisch überprüfen, Kooperationen ablehnen und sich von Muslim:innen, die der Gefahr verdächtigt werden könnten, distanzieren. Die Praxis der gegenseitigen Überprüfung wird von Abdul I. nicht kritisiert; im Gegenteil, er kann sie gut nachvollziehen und hält sie für angemessen, "was ja klar ist«. Aus Angst, für die (vermuteten) Taten Anderer zur Rechenschaft gezogen zu werden, greift die Selbstführung und führt da$\mathrm{zu}$, dass der Verfassungsschutzbericht als Maßstab für das eigene Verhalten innerhalb der Communities dient. Gouvernementalität wirkt, jedenfalls in diesem Kontext, nur in ihrer Interrelation zur Disziplinarmacht. Ohne Androhung von Sanktionen würde die gegenseitige Überprüfung und die Ausrichtung des eigenen Verhaltens an der drohenden Gefährdung der eigenen Gruppe nicht wirken. Abdul I.s Versprecher »Verhaltens- (.) äh ra-Verfassungs- ä:h schutzbericht« und die Beendigung seines Satzes »ist sozusagen unsere Verhaltensgrundlage oder unsere Aktionsgrundlage« reflektiert diesen Zusammenhang: Für muslimische Verbände und andere Zusammenschlüsse, aber auch für Einzelpersonen, ist der Verfassungsschutzbericht nicht lediglich ein Instrument staatlicher Führung, sondern auch ein Instrument der Selbstführung - eine Selbstführung, die zu Entsolidarisierung auch dann führen kann, wenn sie selbst anhand der Kriterien des Verfassungsschutzes nicht gerechtfertigt ist.

Die sicherheitspolitische Unterscheidung zwischen >guten $<$ und >bösen $<$ Muslim:innen im Zusammenhang mit der gouvernementalen Strategie der Selbstführung hat den Effekt, dass Muslim:innen analog dazu vorgehen und muslimische Verbände nach dem gleichen Kriterium zu unterscheiden suchen. Fatouma K. aus der dritten Gruppendiskussion sagt, dass "mit bestimmten Gruppen nicht assoziiert [zu] werden« 
die Chance bietet, zu den >guten Muslim:innen zu gehören und nicht in Ungnade $\mathrm{zu}$ fallen. Diese Einschätzung wird kontrovers diskutiert. Asmah N. beobachtet, dass viele versuchen, möglichen Anschuldigungen dadurch zu entgehen, dass bestimmte Muslim:innen nicht mehr als Referent:innen zu Veranstaltungen eingeladen werden. Es gehört mittlerweile zur gängigen Praxis, muslimische Referent:innen über OnlineSuchmaschinen auszuleuchten, mit Blick auf Details, die als problematisch gewertet werden könnten. Die Diskussionsteilnehmer:innen wissen um diese Details und führen sie im Gespräch nicht näher aus, es handelt sich um eine kollektiv geteilte Erfahrung. Sie können sich also aufeinander beziehen und an das Gesagte anknüpfen, ohne zu explizieren, worum es geht. Von diesem Generalverdacht, auf dessen Grundlage muslimische Gruppierungen andere beobachten, bleiben NichtMuslim:innen verschont. ${ }^{14}$ Dass auch Asmah N. den Auswirkungen des Sicherheitsdiskurses nicht gänzlich entkommen kann, zeigt sich darin, wie auch sie angesichts medialer Berichterstattung über bestimmte Moscheegemeinden und Muslim:innen, die als >Gefährder eingestuft werden, sofort zur Suchmaschine greift und recherchiert: "Ich muss das jetzt wissen.« Trotz ihrer Unterwerfung übt sie Selbstkritik an ihrem eigenen Verhalten. Sie beklagt die Angst vor Kontaktschuldvorwürfen auch innerhalb ihrer eigenen Familie, in der vor dem Besuch von Moscheen gewarnt wird, um sich vor staatlichen und in der Folge auch vor öffentlichen und zivilgesellschaftlichen Verdächtigungen und Sanktionen zu schützen. Asmah N. kritisiert das Ausmaß der Verdächtigungen, sodass muslimische Religionspraxis und das Community-Leben auch intern unter Verdacht stehen, Zeichen einer Radikalisierung zu sein oder als solches missverstanden zu werden. Dass etwa ihre Kinder den Islam- und Arabisch-Unterricht in Moscheen besuchen, wird von anderen misstrauisch kommentiert oder gar davor gewarnt. Die fehlende Abgrenzung kann dazu führen, selbst Entsolidarisierung zu erfahren. Trotz Wissens um den Sicherheitsdiskurs, seiner entsolidarisierenden und grenzüberschreitenden Effekte auf das eigene, das familiä-

14 Obwohl die extreme Rechte sich etwa auf ihr Deutschsein bezieht, geraten nicht alle >Deutschen $<$ unter Generalverdacht. 
re und das Community-Leben und der kritischen Auseinandersetzung damit können sich ihm die Diskussionsteilnehmer:innen nicht entziehen.

\section{"es gibt wirklich manchmal (2) ä Chaos von unserer Seite»}

Die Anrufung, sich von >bösen Muslim:innen abzugrenzen, ist auch in der ersten Gruppendiskussion Thema. Rahman F. ist Imam einer Moscheegemeinde und berichtet von nachbarschaftlichen Problemen, die er lösen will, um ein intaktes Gemeindeleben zu sichern und die Räumlichkeiten nicht zu verlieren. ${ }^{15}$ Er tut dies, indem er den Sicherheitsdiskurs aufgreift, ihn im Zusammenhang mit einer bestimmten Gruppe Muslim:innen bestätigt, die eigene Organisation jedoch davon ausnimmt und so die Gültigkeit des Diskurses nicht grundsätzlich verwirft, aber in Bezug auf sich zu neutralisieren versucht. Der folgenden Sequenz liegt keine Beschreibung eines konkreten Konflikts zugrunde, auf den er sich bezieht; es handelt sich um einen beliebigen, alltäglichen Nachbarschaftskonflikt. Rahman F. räumt ein, dass es "Salafisten « gibt, die »missionieren«, sie »verteilen« Ausgaben des Qur'an in der Stadt. Er erklärt nicht, was problematisch daran ist, Qur'an-Exemplare $\mathrm{zu}$ verteilen und niemand fragt nach, was er meint. Die Markierung als muslimische Praxis knüpft am hegemonialen Wissen an, das allen Diskussionsteilnehmer:innen bekannt ist, unabhängig davon, ob sie es teilen oder nicht.

Das Verteilen von Qur'an-Exemplaren gilt im Rahmen des Sicherheitsdiskurses (und des antimuslimischen Rassismus im Allgemeinen) als problematisch, weil es als Werbung für eine Sache diskursiviert wird, die es nicht zu verbreiten, sondern vielmehr zu begrenzen und einzudämmen gilt. Das Verteilen von Qur'an-Exemplaren symbolisiert vor diesem Hintergrund die >Islamisierung< des Westens und wird als aggressiver Akt des Eindringens und der feindlichen Übernahme interpretiert. Gar nicht in Betracht kommt dagegen eine andere

15 Dieselbe Sequenz wird im Abschnitt Inter_Sektionen unter anderem Fokus interpretiert. 
Deutung, etwa dass es den Gläubigen um Aufklärung über ihre Religion, um Transparenz und Dialog zu offenem und kommunikativem Miteinander in einer pluralen Gesellschaft geht. Diese Deutung liegt auch deswegen in der Diskussion nicht nahe, weil diejenigen, die die Schriften verteilen, als »Salafisten« bezeichnet werden. Salafist:innen gelten als Vertreter:innen eines besonders rückschrittlichen, gewalttätigen und bedrohlichen Islams, insofern ist jede Aktion, die von ihnen ausgeht, $\mathrm{zu}$ missbilligen. Salafiyya als pietistische Reformbewegung $\mathrm{zu}$ verstehen, die auf moderne Herausforderungen antwortet und globalhistorische Bezüge aufweist ${ }^{16}$, kommt als Deutung in dieser Sequenz nicht in Betracht. Rahman F. knüpft am hegemonialen Wissen an und die Diskussionsteilnehmer:innen wissen, was er damit zu verstehen geben will. Insofern muss nicht erwähnt werden, wovon genau sich Rahman F. abgrenzt, um seine Gemeinde als unverdächtig zu präsentieren: Die Symbole >Salafisten und >Exemplare des Qur'an verteilen reichen aus.

Das Bild, das Rahman F. aufruft, ist gesellschaftlich bekannt, wird medial in Szene gesetzt und kann auch unter den Diskussionsteilnehmer:innen als bekannt vorausgesetzt werden. Rahman F. aktualisiert das Bild von den missionierenden Salafist:innen ("Manche haben's ge$\tan \ll)$, um sich tadelnd von ihnen zu distanzieren: »Das ist $\ddot{a}=a ̈$ (1) nicht die Art«. Salafist:innen werden auch im weiteren Verlauf der Diskussion nicht weiter definiert oder ihre Thematisierung wird problematisiert. Die Unterwerfung unter den Sicherheitsdiskurs bestätigt dessen Berechtigung und nimmt die eigene Gemeinde aus. So kann Rahman F. die Fehler der Muslim:innen seiner Gemeinde anders rahmen. Er ruft »Chaos« als Grund für Nachbarschaftskonflikte auf und diskursiviert den Konflikt damit als etwas ganz Normales: »[E]s gibt wirklich manchmal (2) ä Chaos von unserer Seite.« Sich paternalistisch entschuldigend (»wirklich«, »unserer Seite«) steht er zu >seinen Leuten und gesteht Konflikte ein. In der Abgrenzung zu »Salafisten« verschiebt er die Grenzziehung, die im antimuslimischen Rassismus gezogen wird, (>die Muslime versus >die Deutschen`) und bestätigt die Grenzziehung des 
Sicherheitsdiskurses (die >guten versus die `bösen Muslim:innen). Die Unterwerfung unter den Sicherheitsdiskurs geht mit der Entsolidarisierung mit einer anderen muslimischen Gruppe einher.

Die Unterwerfung unter den Diskurs rührt nicht daher, dass seine Gemeinde keine Exemplare des Qur'an verteilt. Vielmehr ist es die Art und Weise, in der er die Distanzierung performativ inszeniert, die auf eine Selbstführung hindeutet: Rahman F. unterwirft sich dem Sicherheitsdiskurs, indem er die Berechtigung der Ausgrenzung >böser Muslim:innen bestätigt, wenn auch mit dem Ziel, seine Gemeinde von der Kritik auszunehmen und diesseits der Grenzziehung anzusiedeln. Gleichwohl deuten seine Ausführungen auch auf Widerstand hin. Widerständig fällt seine Kritik an der negativen medialen Berichterstattung über den Islam im Allgemeinen und das Schüren von Ängsten im Besonderen aus: »[W]ir ä: (2) haben nur Sch- ä Stress mit den Medie(.) ä mit den Medien.« Das inklusive »wir« vereint alle Muslim:innen. Die Differenzierung zwischen >bösen $<$ und guten $<$ Muslim:innen ist angesichts des medialen Islamdiskurses ebensowenig nötig wie jene zwischen verschiedenen Medien. Um diese geht es ihm auch gar nicht, er nennt sie stellvertretend, wenn auch verantwortlich für die öffentliche Meinung und den hegemonialen Diskurs.

Vor diesem Hintergrund ist eine snormale Kontaktaufnahme mit Nachbar:innen nicht möglich: »Die Leute sind nicht genug aufgeklä:rt. Die sind beeinflusst.« Rahman F. kann eine derart vernichtende Kritik an »den Medien« üben und »die Leute« - diesmal sind »die Bürger« gemeint, wie er sie wiederholt bezeichnet - wiederum paternalistisch in Schutz nehmen, weil er die grundsätzliche Legitimität des Sicherheitsdiskurses akzeptiert. Die Unterwerfung durch Entsolidarisierung ermöglicht ihm also sowohl Kritik am hegemonialen Islamdiskurs $\mathrm{zu}$ üben als auch sich vor »seine Leute« und die »Bürger« zu stellen. Im Spannungsfeld von Unterwerfung und Widerstand und unter Berücksichtigung des hegemonialen Wissens kann er seine Kritik formulieren: Es gibt `böseく Muslim:innen namens »Salafisten«, die Bedrohliches tun, und zwar Exemplare des Qur'an verteilen, sodass Kritik an einigen Muslim:innen berechtigt ist. Die Medien verallgemeinern unberechtigterweise und üben Kritik am Islam als Ganzem. Damit schüren 
sie Ängste in der Bevölkerung, sodass normale Nachbarschaftskonflikte vor großen Herausforderungen stehen. Rahman F. unterscheidet nicht zwischen verschiedenen Medienhäusern, Publikationsorganen, Journalist:innen, Textsorten usw. Ihm geht es nicht um die Einschätzung medialer Berichterstattung, sondern um die Skizzierung des hegemonialen Kontextes, in dem Nachbarschaftskonflikte zwischen Muslim:innen und Nicht-Muslim:innen stattfinden. »Die Leute« lassen sich von »den Medien« »beeinflussen «, sodass ein ganz normaler Nachbarschaftskonflikt antimuslimisch oder gar sicherheitspolitisch aufgeladen wird. Die Anordnung von Konflikt und Kontext, die Rahman F. entwirft, ermöglicht es ihm, sein Ziel zu verfolgen, nämlich einen normalen nachbarschaftlichen Konflikt zu lösen bzw. gegebenenfalls dessen Scheitern zu erklären.

\section{"Die reden erst von denen, aber eigentlich meinen die mich."}

Aslı A. aus der ersten Gruppendiskussion beklagt die mangelnde Solidarität untereinander. ${ }^{17}$ Dennoch kann sie die Befürchtungen anderer Diskussionsteilnehmer:innen nachvollziehen, dass es weitere Vorwürfe mit sich bringen kann, als Muslim:innen für andere Muslim:innen $\mathrm{zu}$ sprechen und sich offen für muslimische Themen einzusetzen. Aslı A., Vertreterin eines säkularen Verbandes, bestätigt, dass auch andere muslimische Verbände Herausforderungen zu bewältigen haben, da »man sie der Radikalität bezeichnet«. Der Versprecher (bezeichnet anstelle von bezichtigt) steht für eine symbolische Gleichsetzung von Bezeichnung und Bezichtigung, wonach mit der Bezeichnung als muslimisch bereits der Vorwurf der Radikalität impliziert ist. Im Machtgefälle zwischen Anrufenden und Angerufenen ist die Beziehung zwischen fraglos >Normalen $\triangleleft$ auf der einen Seite und unter Verdacht und Beobachtung Stehenden auf der anderen Seite inhärent. Als radikal »bezeichnet « $\mathrm{zu}$ werden, ist nicht nur einfach irgendeine Bezeichnung, sondern eine Bezichtigung, ein Vorwurf. Der Versprecher weist auf das 
gesellschaftliche Stigma der >Radikalität hin: Die Bezeichnung markiert und wertet im Zuge dessen.

In der dritten Gruppendiskussion provoziert Asmah N., indem sie sich den Begriff »Gefährder « aneignet. ${ }^{18} \mathrm{Zu}$ Beginn zögert sie, schließlich ist ihr die Bedeutung ihrer Aussage bewusst, es sind Personen im Raum, die sie nicht kennt und zwei Aufnahmegeräte zeichnen zuverlässig alles auf, was gesagt wird, auch wenn sie die Stimme senkt. Es entsteht eine subversive Atmosphäre, als sie den Schimpfnamen ins Positive umkehrt und sich implizit solidarisch mit den Beschuldigten zeigt. Die Konstruktion des "Gefährders« überbrückt die Lücke zwischen der Allgemeinheit der Muslim:innen und Personen, die strafrechtlich in Erscheinung treten. Zwischen gouvernementaler und disziplinierender Macht angesiedelt regelt diese Konstruktion nicht nur das Verhältnis zwischen Muslim:innen und dem Sicherheitsapparat, sondern auch unter den Muslim:innen selbst. Die Unbestimmtheit des Gefährder-Begriffs ragt in muslimische Communities hinein, verunsichert sie und gefährdet Kooperationen. Asmah N. erklärt den anwesenden Diskussionsteilnehmer:innen, dass alle im Raum und auch andere, die sich aktivistisch betätigen, jederzeit als >Gefährder:in< klassifiziert werden können und viele gar nicht wissen, dass ihr Kooperationspartner oder gar sie selbst als solche unter Beobachtung stehen: »Vielleicht arbeiten sie aktivistisch zusammen, weil die Leute das selbst nicht wissen.« Asmah N. beschreibt das Problem, dass Menschen, die als >Gefährder gelten, ohne es zu wissen, auch andere gefährden, die fortan mit ihnen assoziiert werden. Sich zivilgesellschaftlich $\mathrm{zu}$ engagieren, kann also auch jene gefährden, die vom Engagement profitieren, weil sie mit >Gefährder:innen in Verbindung gebracht werden könnten. Das Thema beschäftigt alle im Raum, Asmah N.s Ausführungen werden nur durch gemurmelte (»((zustimmend))«) oder deutliche (»@Voll!@@()@«) Zustimmung, Nachfragen und Zwischenrufe (« ((wirre und lebhafte Gespräche durcheinander))«) und nachdenkliche Laute (»ja, hm:, ((aktiv zuhörend))«) unterbrochen.

18 Diese Sequenz wird im Abschnitt Ver_Antworten unter anderem Fokus diskutiert. 
Auch Fatouma K. stimmt mehrfach zu (»Und man meint uns, ja.«) und will Genaueres wissen: ${ }^{\circ}$ Ich versteh das grade nicht. ${ }^{\circ}$ Wer=wer soll- oder wer hat diesen Begriff überhaupt gesetzt, Gefährder? « Entsetzen, Sorge und Verunsicherung einen die Gruppe. Alle sprechen leise, sodass der Eindruck entsteht, als fühlten sie sich beobachtet und abgehört, als sprächen sie über etwas Verbotenes, das ihnen Schaden zufügen könnte. Auf Fatouma K.s Nachfrage antwortet Asmah N. direkt mit »Polizei«; auch sie flüstert zurück, es sei eine »Polizeifachdefinition« und bestätigt damit die Atmosphäre. Während einige im Raum eine Antwort auf die Frage suchen, wer den Begriff etabliert hat - Tasnim J. bringt das Innenministerium fragend ins Gespräch und Asmah $\mathrm{N}$. führt Landeskriminalämter und das Bundesverwaltungsgericht als Institutionen auf, die den Begriff übernahmen - beschäftigt Leyla L. das Wort selbst; sie empfindet es als »hart beleidigend «. Der Begriff als solches, die Zuschreibung und Bezeichnung als Gefahr, stellt eine Beleidigung dar, die zudem »hart « ist, sie verletzt und erniedrigt. Sie ist keine Gefahr und will auch nicht als solche tituliert werden. Leyla L. spricht aber nicht nur für sich selbst, sondern »find[et] das Wort schon hart beleidigend« und bezieht ihre Kritik daran auf die Bezeichnung von Muslim:innen im Allgemeinen (oder gar aller Menschen) als >Gefährder . Im Verlauf der gesamten Sequenz verteidigt sich niemand, alle teilen das situierte Wissen, dass die Anschuldigung haltlos ist. Eine Unterwerfung kommt nicht in Frage, auch nicht in der Form, sich selbst von der Anschuldigung auszunehmen. Obwohl Leyla L.s Resümee gegen diejenigen gerichtet scheint, die den Begriff prägen und nutzen, weist er in die Community hinein und stellt eine widerständige Solidaritätsbekundung dar: Die grundsätzliche und unspezifische Zurückweisung der pejorativen Bezeichnung >Gefährder als »hart beleidigend « unterscheidet nicht zwischen Muslim:innen, die ihn verdienen und anderen, die fälschlicherweise erniedrigt werden. Die selbstverständliche und übereinstimmende Ablehnung vereint die Gruppe, sie solidarisieren sich nicht nur untereinander, sondern auch mit anderen (potentiell allen) Muslim:innen, da diese ebenfalls von der Beleidigung betroffen sind. Die Funktion des Gefährder-Konzepts, auch unbescholtene Muslim:innen als potentielle Straftäter:innen auszuspä- 
hen, sie zu verunsichern und Misstrauen $\mathrm{zu}$ sähen, greift jedenfalls in diesem Moment nicht: »Die reden erst von denen, aber eigentlich meinen die mich.«

Asmah N. spricht das diskursive Verbot aus: Sie identifiziert sich mit den >Gefährder:innen`. Die Identifikation mit ihnen gibt eine ambivalente Verortung im Diskurs wieder, in der sich Asmah N. (und mit ihr die anderen) angesichts des Bedrohungsszenarios als `Gefährder:innen〈 wiederfinden bzw. befürchten, aufgrund undurchsichtiger Kriterien als solche beobachtet, kontrolliert und reglementiert $\mathrm{zu}$ werden. Asmah $\mathrm{N}$. nimmt den Namen an, aber nicht um sich selbstführend als >guteく Muslimin von den >bösen` `Gefährder:innen` abzugrenzen, sondern sie nimmt ihn für sich selbst an und fordert die Deutungshoheit über die Begrifflichkeiten heraus. Sie tritt in den Diskurs ein, will aber die Spielregeln mitbestimmen, den Diskurs durch kritische Analyse und Aufklärungsarbeit mitgestalten und beginnt gleich in der Gruppendiskussion damit. Sie nimmt die Beleidigung nicht an, auch nicht indem sie dagegen rebelliert oder sie zurückgibt. Vielmehr akzeptiert sie den Namen und nutzt ihn zur solidarischen Selbstermächtigung: Wenn alle Muslim:innen Gefährder:innen sind, verliert das Konzept seine Macht. Obwohl sich diese Strategie von der Zurechtweisung (»hart beleidigend«) unterscheidet, deuten beide auf die Solidarisierung mit jenen hin, die als >Gefährder:in< bezeichnet und bezichtigt werden, und auf das widerständige Potential, das in der Solidarisierung liegt.

\section{»ich bin hier groß geworden. Ich bin hier geboren."}

Auch bei guter Führung, also Unterwerfung unter den Sicherheitsdiskurs und kritikloser Annahme der zugewiesenen Position, bleibt die gleichberechtigte Anerkennung aus, so Gülçin D. aus der ersten Gruppendiskussion. Die Gleichstellung mit anderen Deutschen ist prekär und kann jederzeit einseitig aufgelöst werden. Dazu reichen Kleinigkeiten aus, die dazu dienen, das Gegenüber zu muslimisieren und zur Gefahr zu erklären. Gülçin D. kritisiert, dass man »bei einer kleinen Sache [...] trotzdem der Muslim« ist, so sehr man sich auch anstrengt, den zugewiesenen Platz zu akzeptieren und dabei »nett« und »freundlich« 
bleibt. ${ }^{19}$ In einer späteren Sequenz bezieht sich Gülçin D. erneut auf das Othering von Muslim:innen. Ihrem Erfahrungshorizont entsprechend spielt die Arbeit eines türkischen Verbandes eine zentrale Rolle. `Gute Muslim:innen, die zuvor allseits als Kooperationspartner bevorzugt wurden (»hätte man mit keinem anderen Muslim zusammen gearbeitet«), wurden plötzlich fallengelassen und unter Beobachtung gestellt: Sie stehen jetzt »auch auf dieser ä:hm auf diesem Bedrohungs- (2) auf der Bedrohungsliste. Ne, es sind auch die bösen Muslime geworden.« Die Homogenisierung und das Fremdmachen von >Muslim:innen geht so weit, »deutschtürkische Muslime«, zu denen sie sich selbst zählt, für türkische Regierungspolitik in die Verantwortung zu ziehen. Jahrzehnte lange gute Kooperation kann "mit einer Türkeipolitik [...] auch das schon wieder zu-ä-nichtewerden«. Gülçin D. lässt hier offen, wen sie für schuldig hält an der Transformation von sguten Muslim:innen in >böse und für die sicherheitspolitische Überwachung (»Bedrohungsliste«). Sie führt auch nicht weiter aus, was vorgefallen ist und es bleibt unklar, ob sie die deutsche »Türkeipolitik«, jene des Verbandes oder die türkische Politik meint. Ihre Solidarität ist indes eindeutig und gilt ihrer Bezugsgruppe: „Das sind ja Leute, die hier groß geworden sind, deren Eltern mit diese Moscheen aufgebaut haben, die in den Projekten arbeiten, das sind ja wir hier, die deutschtürkischen Muslime.«

Die Solidarisierung mit den nun >bösen Muslim:innen hält Gülçin D. nicht davon $a b$, ihnen »Fehler $z u$ attestieren: Sie haben, so meint sie, »sich ja auch viele Fehler erlaubt«. Ihre Solidarität ist nicht unkritisch, aber angesichts der Sanktion eine notwendige Folge. Sie grenzt sich von anderen Muslim:innen ab, die nicht in ihrem »wir « mitgemeint sind. Sie selbst betont mehrfach: »Ich bin hier groß geworden. Ich bin hier geboren. « Ihr verzweifelter Ausruf ist in Richtung Dominanzgesellschaft gerichtet und zielt darauf, die gleichen Rechte wie alle anderen auch, und zwar »als Muslimin in dieser Gesellschaft« zu haben. Das geschieht in Abgrenzung zu nicht-türkischen Muslim:innen und

19 Diese Sequenz wurde unter anderem Fokus im Abschnitt Inter_Sektionen diskutiert. 
$\mathrm{zu}$ nicht hier geborenen und nicht hier aufgewachsenen deutschtürkischen Muslim:innen. Im Zusammenhang mit der »Türkeipolitik«steht ihre Zugehörigkeit jenen gegenüber, die sie als »Imame, Attachés, Bürokraten« bezeichnet und von denen sie sich vehement abgrenzt. Gülçin D. unterwirft sich damit dem Sicherheitsdiskurs und folgt der nationalen Einteilung: >Gute Muslim:innen sind hier geboren, >böse dringen von außen ins Land ein und gehören einem anderen Staat an, sind gar dessen Vertreter. Sie bedient sich des Diskurses über einen domestizierten >Euro-Islam< in Abwehr eines anderen, fremden, >bösen $<$ Islams. Dieser trägt nicht nur seine Interessen von außen in die deutsche Gesellschaft hinein, sondern bedroht ihren Platz darin. Gülçin D. betont, dass sie »hier« in Deutschland geboren ist und das Recht hat, als »Teil in dieser Gesellschaft« anerkannt zu werden. Über die Türkei verliert sie bis auf die genannten Kontexte, in denen es um Schuldige (»Türkeipolitik«) und Eindringlinge (»Imame, Attachés, Bürokraten«) geht, kein Wort. Ihre Solidarität ist eindeutig, sie gilt nicht allen Muslim:innen, im Gegenteil: Einige gefährden ihr Deutsch(türkisch)sein und werden aus der Solidarisierung implizit oder explizit ausgenommen. Die hegemoniale Gleichsetzung von Religionszugehörigkeit und nationaler $\mathrm{Zu}$ gehörigkeit wird bestätigend verschoben: »Deutschtürkische Muslime« gehören dazu, andere Muslim:innen gefährden dieses Arrangement. Das Changieren zwischen Solidarisierung und Entsolidarisierung geht einher mit der punktuellen Unterwerfung unter den Sicherheitsdiskurs, die es ihr erlaubt, Kritik zu üben und die Grenzen, wenn auch nur zu Gunsten ihrer eigenen Gruppe, zu verschieben.

\section{"der war ja nicht religiös«}

In der ersten Gruppendiskussion unterscheiden Gülçin D. und Rahman F. (auf unterschiedliche Weisen) zwischen sguten lim:innen. Sie normalisieren die sguten Muslim:innen, indem sie ihr Muslimischsein unmarkiert lassen und implizit als snormak mitführen: Gülçin D. spricht von "ga:nz norma:le[n] Jugendliche[n] « mit Bedarf nach »Jugendarbeit« und »religiöser Unterweisung«. Von ihnen grenzt sie die »radikalisierten jungen Leute $[n] \ll a b$, die sie auch als »ra- 
dikalisierte Muslime« bezeichnet. Rahman F. unterscheidet zwischen "Salafisten « und »unseren Leuten«: Während erstere eindeutig aus dem Rahmen fallen, werden letztere durch ihre fehlende muslimische Rahmung normalisiert. Die fehlende Bezeichnung des Selbstverständlichen und Normal(isiert)en ist im Kontext hegemonialer Benennungspraktiken bekannt, die explizite Bezeichnung von Menschen als weiß, bürgerlich oder heterosexuell stößt bei diesen auf Irritation; sie bleiben üblicherweise unmarkiert. Gülçin D. und Rahman F. bedienen sich dieser Strategie, um sich und ihre Leute als >normale Menschen zu empfehlen und quasi leise hinzuzufügen, dass es sich um Muslim:innen handelt.

Die Entsolidarisierung gegenüber den `bösen` >Muslim:innen geht nun paradoxerweise mit ihrer Hypervisibilisierung als (>böse $)$ Muslim:innen bei gleichzeitiger Entmuslimisierung einher. Das funktioniert am Beispiel von Gülçin D. folgendermaßen: Sie bezieht sich auf den berechtigten Bedarf "ganz normaler Jugendlicher« nach »religiöser Unterweisung«. Sprachlich stellt sie muslimische Jugendliche mit christlichen, jüdischen, buddhistischen und anderen Jugendlichen gleich. Trotzdem besteht kein Zweifel, dass es ihr um Muslim:innen geht. Die Begriffsverschiebung, die das Muslimische in den Hintergrund rückt und dafür das Religiöse und das Jugendliche aufruft, greift anerkannte Aspekte auf und nutzt sie für das Ziel, muslimische Religionszugehörigkeit als eine mögliche von sganz normalen Deutschen zu normalisieren. Die performative Wiederholung erlaubt ihr, ihre Interessen als Bürger:in zu artikulieren (»Ich bin ein Teil in dieser Gesellschaft. Ich möchte mich nicht andauernd erklären «) ${ }^{20}$ und im Zuge dessen, ihre Religion zu inkludieren (»Ich möchte, dass es selbstverständlich ist, dass ich als Muslim in dieser Gesellschaft (1) ((atmet tief ein)) genauso viel Rechte habe«).

Gleichzeitig geschieht dies auf Kosten der Exklusion anderer Muslim:innen, und zwar aus der Gemeinschaft der Muslim:innen: Zunächst spricht Gülçin D. von »radikalisierte[n] Muslime[n]«, dann von »radikalisierten jungen Leuten«, anschließend argumentiert sie bezüglich 
eines konkreten Falls, den sie verallgemeinert: »[D]er war ja nicht religiös, ja, es geht ja, also (2) wenn er religiös gewesen wä:re, und, hm (2), wie sagt man (1) aufgeklärt, dann würde er ja wissen, dass er sowas nicht machen darf oder nicht machen kann. "Sie bezieht sich auf den Fall eines Jugendlichen, der einen Mann wegen seiner Kippa mit einem Gürtel schlug. Der im öffentlichen Diskurs als muslimisch markierte Täter wird von ihr als nicht-religiös jenen "ganz normalen Jugendlichen « entgegengehalten, deren religiöse Unterweisung zum Ziel hat, sich das Verhalten des Propheten zum Vorbild zu nehmen. Der Gewalt des entmuslimisierten Jugendlichen stellt sie das angestrebte Verhalten normalisiert-muslimischer Jugendlicher gegenüber: »[E]in vorbildlicher, ein liebevoller, ein respektvoller Umgang mit anderen Menschen« ist für sie das Ziel der »religiösen Unterweisung«. Die Unterwerfung unter den Sicherheitsdiskurs erlaubt es ihr, eine Verschiebung vorzunehmen: Das gewalttätige Verhalten, das im antimuslimischen Diskurs als muslimisch markiert wird, wird von ihr als nicht-muslimisch, weil nicht religiös gedeutet. Sie lehnt die Gewalt ab, weil sie Muslimin ist (und nicht obwohl sie Muslimin ist) und erwartet, "als Muslimin « anderen Deutschen gleichgestellt zu werden. Die Unterwerfung erlaubt den Widerstand gegen antimuslimische Stereotype und überlässt die entmuslimisierten Jugendlichen dem Sicherheitsdiskurs, sodass die Durchführung von Deradikalisierungs- und Präventionsmaßnahmen als gerechtfertigt erscheint, wenn auch nicht für normale (also muslimische) Jugendliche indiziert.

\section{"Ok, dann machen wir halt äh unser Ding“}

In der zweiten Gruppendiskussion wird die Frage der Ent_Solidarisierung mit Blick auf Konflikte zwischen etablierten Muslim:innen thematisiert, die in Deutschland »Strukturen« aufgebaut haben und sich darin engagieren, und jüngeren, »in Deutschland sozialisiert[en]« Muslim:innen, die neue Strukturen aufbauen - teils neben, teils gegen die alten Strukturen. Abdul I. kritisiert dies scharf und bevorzugt es, auch konfliktreiche Diskussionen zu führen und »Widerstände« als »interne« anzugehen und $\mathrm{zu}$ »überwinden«, um muslimische 
Strukturen zwar divers, aber gemeinsam zu gestalten. »Ich glaube schon, dass wir neue Initiativen und so brauchen, aber sie müssen nicht diesen (.) diese Richtung einschlagen. « Er kritisiert, dass junge, hier sozialisierte Muslim:innen es sich durch den Austritt aus den etablierten Verbänden zu leicht machen, wenn sie den Konflikten ausweichen. Abdul I. spricht aus der Position der alten Strukturen, die er repräsentiert und denen als Strukturen eine gewisse Starrheit, aber auch Stärke anhaftet. Etwas daran ändern zu wollen, bedeutet für ihn, gegen »Widerstände« ankämpfen zu müssen, von denen er aber glaubt, dass sie »zu überwinden« sind. Er führt verschiedene Gründe an, warum aus seiner Perspektive die Auseinandersetzung zu suchen ist. Zum einen gebietet das laut Abdul I. der Respekt gegenüber jenen, die die Strukturen aufgebaut haben. Er wünscht sich, dass neue Vereine »sagen >Okay, wir sind im Dialog, wir respektieren die Arbeit der Verbände usw.« Er kritisiert also nicht grundsätzlich die Gründung neuer Organisationen, sondern seine Kritik richtet sich dagegen, dass neue Vereine in Opposition $\mathrm{zu}$ den alten Strukturen gegründet werden und den internen Konflikten ausweichen, mehr noch, sie in der Öffentlichkeit austragen, die also »sagen >Ja wir sind gegen Verbände und wir kritisieren sie jetzt von $\mathrm{A}$ bis $\mathrm{Z}$ und sonst was ähm und wir arbeiten sogar äh im Zweifelsfall gegen sie.«

Aus Abdul I.s Sicht ist das respektlos. Das Vorgehen ist nicht nur aus moralischen Gründen, sondern seines Erachtens auch deswegen verwerflich, weil die neuen Vereine aus den alten Strukturen hervorgingen. Ohne die Stärke, den Rückhalt und die Unterstützung durch die etablierten Strukturen wären die neuen Akteur:innen nicht in der Lage gewesen, sich zu "akademisieren « und Neues aufzubauen, so seine Argumentation. "Die sind aus den Strukturen heraus entstanden, aus den Strukturen heraus. "Samir H. stimmt Abdul I. zu, sie reden durcheinander und bestätigen sich gegenseitig: »Ja, genau, [...] das geworden, was sie sind und propagieren jetzt etwas VÖLLIG NEUES, NIE DAGEWESENES. «Er führt seinerseits aus: „Es DARF äh außerhalb der ähm (3) der Moscheen oder der Strukturen das geben, aber diese Leute, die sich strukturiert haben, müssen eben selber sich fragen >Welchen Mehrwert gebe ich der muslimischen Community insgesamt zu- 
rück.« Samir H. ist ebenfalls nicht grundsätzlich gegen Neugründungen und eine Pluralität von Vereinen und Verbänden. Er erwartet allerdings nicht nur Respekt, sondern auch Dankbarkeit sowie eine gewisse Bescheidenheit in der Einschätzung der eigenen Arbeit.

In der Argumentation der beiden Vertreter etablierter Verbände scheinen die klassischen Konflikte zwischen etabliert und innovativ, Eltern und Kindern und ähnlichen Konstellationen durch. Im Unterschied zu den üblichen Konflikten dieser Art finden die hier genannten jedoch in einem gesellschaftlichen Kontext statt, der muslimische Verbände beobachtet, kommentiert und reglementiert. Abdul I. meint: »Ja, das das ist ja (.) offensichtlich ne Tendenz ähm, die die mehr als gefährlich ist aus meiner Sicht.« und Samir H. bestätigt: »Das sehe ich auch.«Die eindringliche Aufforderung, die Konflikte als »interne« zu behandeln und die »Community-Anbindung « nicht aufzugeben, reflektiert den gesellschaftlichen und sicherheitspolitischen Kontext. Auch die Erinnerung daran, dass die hier sozialisierte Generation durch die Stärke, den Rückhalt und die Unterstützung der Communities wachsen konnte, reflektiert den Umstand, dass die Marginalisierung von Eingewanderten aufgefangen wurde und die Verbände durch eigene Angebote der Diskriminierung entgegen getreten sind. Vor diesem Hintergrund stellt die öffentliche Opposition, also das Hineintragen interner Konflikte in jene Bereiche der Gesellschaft, die die Entfaltung von Muslim:innen behindern, eine besonders empfindliche Verletzung dar und hat Verständnislosigkeit und Kränkung zur Folge. Die hier sozialisierte Generation, so Abdul I., reflektiert diesen Umstand nicht. Sie bricht das Gespräch mit der Community ab und wendet sich der Dominanzgesellschaft zu.

Die Kommentierung der oppositionellen Neugründungen erfährt aber auch aus einem weiteren Grund Kritik. Abdul I. unterscheidet verschiedene Positionierungen innerhalb der muslimischen Community und führt Alter, Sozialisation, »liberale Muslime« und »MainstreamMuslime« an. Er hält der - aus seiner Sicht - »fragwürdige[n] Entwicklung« den Vorschlag entgegen, »interne Widerstände zu überwinden« und »neue Initiativen« respektvoll und im Dialog mit den alten Strukturen zu gründen. Diesen Umgang miteinander und mit Konflikten ver- 
misst er: Er bedauert, dass »das immer mehr verloren geht« und fragt sich, »wo das am Ende hinführen wird«. Seine Kritik richtet sich auch gegen die Beliebigkeit und Unverbindlichkeit jener Gruppierungen, die er - analog zu deren Selbstbezeichnung - unter einem »liberalen Label« zusammenfasst. Aber auch »Mainstream-Muslim:innen« wirft Abdul I. Kompliz:innenschaft vor. Es handelt sich bei einer der Gruppen, über die in der Diskussion exemplarisch geredet wird, laut Abdul I. um "20 Männekens«, die aber große öffentliche Aufmerksamkeit erhalten, ohne seiner Meinung nach repräsentativ sein zu können. Aus der Perspektive der etablierten Strukturen ist das seiner Meinung nach "gefährlich«, und zwar "gesellschaftlich« und »intern muslimisch«. Samir H. verknüpft den Vorwurf der Beliebigkeit mit dem Argument der Gefährlichkeit und meint, dass »eine selbst gewählte Optimierung höchst gefährlich« ist. Gegen Neugründungen hat auch er nichts einzuwenden, erwartet von den Akteur:innen aber eine Orientierung an muslimischen Interessen: "Es DARF äh außerhalb der ähm (3) der Moscheen oder der Strukturen das geben, aber diese Leute, die sich strukturiert haben, müssen eben selber sich fragen: Welchen Mehrwert gebe ich der muslimischen Community insgesamt zurück. « Er wird dabei mehrfach von Abdul I. bestätigt: »Genau!« An Abdul I.s einleitenden Ausführungen anknüpfend beklagt Samir H., dass die kritisierten Vereine sich an persönlichen, individuellen Interessen und an der Optimierung der eigenen Person und Position orientieren. Im Zuge der »selbstgewählten Optimierung« verlieren seiner Ansicht nach diese Neugründungen die Community aus dem Blick, da ihre Aktivitäten keinen »Mehrwert« für Muslim:innen haben. Samir H. hält die dominanzgesellschaftliche Anerkennung dieser Gruppen für »gefährlich«, weil »20 Männekens« ohne "Community-Anbindung« als repräsentativ missgedeutet werden. Indem diese nicht nur neben, sondern auch gegen die etablierten Strukturen arbeiten, sind sie auch »intern muslimisch« "gefährlich« und schädigen die Community: «[G]egen die Strukturen, ähm äh und auch ähm ZU DEM PREIS, DASS WIR DIE STRUKTUREN SCHWÄCHEN. Und ich glaub das ist ne=ne fr- ne sehr fragwürdige Entwicklung."

Obwohl einige Kritikpunkte gegen das attestierte neoliberale Verhalten gerichtet sind, meinen die Diskussionsteilnehmer:innen hier 
nicht jene, die sich unter dem »liberalen Label« formieren. Im Unterschied zu »liberalen « Muslim:innen, über die es sich - so die einhellige Meinung - nicht lohnt weiter zu sprechen, stellt Samir H. heraus, dass der Verein, über den diskutiert wird, aus den Strukturen heraus entstanden ist. Er wiederholt diese Tatsache mehrfach. Die Kränkung liegt darin, dass die Akteur:innen des Vereins auf eine gemeinsame Geschichte mit jenen Muslim:innen und ihren Strukturen zurückblicken, von denen sie sich nun absetzen. Der genannte neue Verein greift, so die Kritik der Diskussionsteilnehmer:innen, auf geteiltes Wissen zurück, stellt dieses aber als neu dar, grenzt sich von den etablierten Strukturen $\mathrm{ab}$ und setzt sich medienwirksam in Szene. Für Unverständnis unter den Diskussionsteilnehmer:innen sorgt auch, dass diese Gruppe im Unterschied zu den »liberalen« Muslim:innen weiß, wovon sie spricht. Während mit den »liberalen« Muslim:innen keinerlei Verbindung und demnach auch keine Solidarität, noch nicht einmal eine aktive Entsolidarisierung stattfindet, scheint das Band zwischen den etablierten Verbänden und den neuen Vereinen zumindest in Form einer aktiven gegenseitigen Abgrenzung noch zu bestehen. Aufgrund ihrer historischen und religiösen Nähe kann aus Sicht der alten "Strukturen« ein solidarisches Mit- oder auch Nebeneinander nur aufrechterhalten werden, wenn ein Mindestmaß an Respekt, Vernetzung und Community-Orientierung gegeben ist. Dazu gehört auch, dass die Arbeit der jeweils anderen anerkannt wird und nicht "propagiert « wird, dass die neuen Vereine etwas "völlig Neues«, "nie Dagewesenes« anbieten. Anstatt konkrete, niedrigschwellige Vereinsarbeit zu leisten, die an den Bedürfnissen der Community ansetzt, organisieren die Akteur:innen der neuen Vereine aber zum Beispiel eine Konferenz, die an eine nicht-muslimische Zielgruppe gerichtet ist. Das wertet Samir H. als »Versuch der Akademisierung«. Anstatt der Gemeinde zu dienen und ihr etwas zurückzugeben - schließlich haben auch sie von den Gemeindediensten profitiert -, bemühen sie sich um Nicht-Muslim:innen, und zwar auf Kosten jener Community, die sie aufgezogen hat, so Samir H. Die Aufkündigung der Solidarität seitens der neuen Vereine wird ihrerseits mit Entsolidarisierung beantwortet. In der Argumentation deuten sich mehr als nur eine Konkurrenzsitua- 
tion sowie gegenseitige Verletzungen und Kränkungen an, vielmehr scheint hier ein klassischer Eltern-Kind-Konflikt durch. Der Kontext ist jedoch gesellschaftlich strukturiert, der Vorwurf des fehlenden "Mehrwerts« und mehr noch, der Arbeit "gegen die Strukturen«, um den Preis, diese zu »schwächen", erhält angesichts dessen eine gesellschaftliche Dimension von großer Reichweite.

Die sicherheitspolitische Dimension des Konflikts liegt darin, dass das Ausrichten von öffentlichkeitswirksamen Angeboten, die sich an den Interessen der Dominanzgesellschaft orientieren, politisch und finanziell gefördert wird, während die allägliche Basisarbeit als Ehrenamt geleistet wird und unsichtbar bleibt. Während geförderte Vereine öffentlichkeitswirksame Konferenzen für Nicht-Muslim:innen planen, muss sich der Moscheevorstand nach Feierabend »um Klopapier kümmern [...], um den Wasserrohrbruch und dann soll er noch ein Konzept für Jugendarbeit entwerfen?«, so Samir H. Mit Blick auf die Privilegien und Ressourcen der anderen verteidigt er sich und entschuldigt die wenig aufregenden Angebote: »Was bieten wir denen an?« Hier scheint der Konflikt zwischen produktiver und reproduktiver Arbeit durch. Samir $H$. ist verärgert, weil die ausscherenden Akteur:innen der neuen Vereine all das wissen, schließlich sind sie in diesen Strukturen großgeworden. Umso unverständlicher ist ihm ihre Kritik an den »Leute[n] in der Moschee«. Samir H. kritisiert, dass sie Moscheeverbände öffentlich diskreditieren, anstatt der Community etwas zurückzugeben.

Rania G., der Jüngsten in der Runde, gelingt es nach wiederholten Versuchen, in das Gespräch einzusteigen. Als Vertreterin eines muslimischen Jugendverbandes macht sie auf die Erfahrungen von muslimischen Jugendlichen aufmerksam, um sowohl die Achse zwischen Jung und Alt aufzubrechen als auch der Deutung des Konflikts eine andere Sichtweise hinzuzufügen. Sie fordert von den älteren Anwesenden als Vertreter der etablierten Strukturen Unterstützung ein und kritisiert ihrerseits deren fehlende Würdigung des Engagements der Jungen, die innerhalb der Community aktiv sind: »[G]anz ehrlich, also bei uns inim Verband, wer sind denn die Aktiven? Studenten. Und warum? @Weil die flexibel sind.@.«Sie durchkreuzt damit gleich mehrere Differenzlinien, die Samir H. und Abdul I. in ihrer Kritik angeführt hatten: $\mathrm{Zu}$ - 
nächst ist ihr Verband ein Beispiel dafür, dass jüngere, hier sozialisierte Muslim:innen innerhalb der Strukturen aktiv werden. Der Markierung der Neugründungen als »Versuch der Akademisierung« hält sie entgegen, dass in ihrem Verband angehende Akademiker:innen (»Studenten«) die Hauptlast tragen. Argumentativ setzt sie der Identifizierung von Gründen für die reproduktive Arbeit eine andere Komponente als jener von Samir H. angeführten entgegen: Personen, die über Zeit verfügen, können sich um die Basisarbeit kümmern. Insbesondere aber steht die Gruppendiskussion selbst paradigmatisch für den Umgang mit jüngeren Mitgliedern der Community: Rania G. wird nicht gehört. Selbst als sie in dieser Sequenz endlich sprechen kann, wird ihr Beitrag von Abdul I. kurz kommentiert (»Sicherlich. Gar keine Frage.«) und das Gespräch in eine andere Richtung weitergeführt. Rania G. leitet ihren Redebeitrag damit ein, dass sie das Gespräch zum Thema der Diskussion zurückführen und daran erinnern will:

»]a, aber einfach nochmal um zu=zu dem Thema Sicherheitsdiskurse zurückzukommen. Man muss aber auch sagen, dass eben, also ich mach Sicherheitsdiskurse auch mit dafür verantwortlich, dass ähm Jugendarbeit oder islamische Verbandsarbeit whatever, ähm (.) ein Privileg bleibt.«

Sie führt also ebenfalls den gesellschaftlichen Kontext in die Reflexion ein, gibt diesem aber eine andere Wendung. Während Samir H. und Abdul I. die fehlende Berücksichtigung des Kontextes im Zusammenhang mit dem öffentlichen Austragen interner Konflikte bemängeln, argumentiert Rania G. diesbezüglich mit Blick auf muslimische Verbände insgesamt. Fehlende Ressourcen für die Basis- und Verbandsarbeit erweitert sie um fehlende Ressourcen für Jugendarbeit und lenkt damit die Kritik um: Anstatt gegeneinander zu argumentieren und sich um die viel zu knapp bemessenen Ressourcen zu streiten, richtet sie ihre Kritik an diejenigen, die die Verteilung von Ressourcen verantworten. Die Entsolidarisierung, die im Gespräch zwischen Samir H. und Abdul I. tragend ist, wird von Rania G. aufgefangen und damit implizit 
Solidarität eingefordert, um gemeinsame Interessen verfolgen zu können. ${ }^{21}$

Rania G. ruft in ihrer Argumentation in Erinnerung, dass die Sicherheitsdiskurse maßgeblich für die Handlungsfähigkeit von muslimischen Verbänden und Organisationen verantwortlich sind, etwa wenn Gemeinnützigkeit aberkannt und der Zugang zu finanziellen Ressourcen eingeschränkt oder gar nicht erst gewährt wird. Ehrenamt kann dann häufig nur von flexiblen und aktiven Studierenden getragen werden. Rania G.s Argument beleuchtet Samir H.s Kritik aus einer anderen Perspektive: Sie reagiert (ähnlich zur ersten Interviewsituation zwischen Gülçin D. und Rahman F.) auf die Erwartungshaltung der älteren Generation ihrerseits mit der Aufforderung, das Engagement der jungen Generation zu würdigen und ihre Lebenssituation sowie ihre Interessen im Kontext des Sicherheitsdiskurses anzuerkennen. Sie lenkt den Blick auf die Erfahrung anderer junger muslimischer Studierender und Ehrenamtlicher, die Lücken schließen, aber unsichtbar gemacht und nicht gehört werden. Der Entsolidarisierung setzt sie einen Appell zur Solidarisierung entgegen und fordert gemeinsames Handeln ein, um der gesellschaftlichen Diskriminierung begegnen zu können. Auch in den anderen Gruppendiskussionen wird anhand konkreter Beispiele und allgemeiner Diskussionen darüber gesprochen, wie der gesellschaftliche Islam- und Sicherheitsdiskurs in das Verhältnis zwischen Muslim:innen hineinragt und dabei Fragen nach Ent_Solidarisierung zentral sind. Denn die Sichtbarkeit von Muslim:innen als Muslim:innen in der Öffentlichkeit ist auf wenige ihnen zugestandene Rollen begrenzt, die zudem eher dazu geeignet sind, sich von anderen (als) Muslim:innen (Markierten) abzugrenzen als muslimische Bedürfnisse aufzugreifen. Zusammengenommen verdeutlichen die Argumentationen, wie komplex und vielschichtig die Fragen sind, mit denen sich die Verbände auseinandersetzen müssen, und dass dabei sowohl intersektionale Machtverhältnisse als auch die Rahmung durch den Islam- und Sicherheitsdiskurs stets mitverhandelt werden.

21 Über Jugendarbeit ist im Abschnitt Ver_Antworten mehr zu lesen. 


\section{Ver_Antworten}

In allen Gruppendiskussionen antworten die Diskussionsteilnehmer:innen auf den Sicherheitsdiskurs, indem sie den Staat zur Verantwortung ziehen und ihn mehr oder weniger explizit auffordern, demokratischen Prinzipien gerecht zu werden. Dazu gehört aus ihrer Sicht selbstverständlich auch, muslimische Interessen und Bedürfnisse als bürgerliche in einem säkularen Staat zu akzeptieren. Sie diskutieren verschiedene Konstellationen, in denen sie ihre Anerkennung und ihre Sicherheit gefährdet oder missachtet sehen. Die Funktionsweise des >Bedrohungsszenarios « wird evaluiert hinsichtlich seiner Auswirkungen auf ihr persönliches und soziales Leben, ihr zivilgesellschaftliches Engagement und ihre Gemeindearbeit sowie ihren beruflichen Alltag. Sie kehren also die Blickrichtung um: Die geteilte Erfahrung, beobachtet, kommentiert und reglementiert $\mathrm{zu}$ werden, wird selbst zum Gegenstand von Beobachtung und Kommentierung. Unsere Diskussionsteilnehmer:innen reflektieren die Adressierung von >Muslim:innen als Bedrohung unter Berücksichtigung intersektionaler Konstellationen ${ }^{22}$ und ambiguer Bedeutungen für jeweils konkrete Kontexte. Sie werden gleichzeitig als Teil des Problems und seiner Lösung adressiert und durch wiederholte Ansprachen dazu angehalten, sich zum Sicherheitsdiskurs zu positionieren. Sie werden zur Distanzierung von >Islamismus< und >islamisch legitimierter Gewalt aufgefordert, ansonsten droht die Disziplinarmacht in Form von Verdächtigung, Beobachtung und Reglementierung zu greifen sowie damit, Restriktionen und Sanktionen zu ergreifen. Ihr aktiver Beitrag, den sie als Muslim:innen zur Gesellschaft leisten, wird auf die Mitwirkung in Maßnahmen zu Deradikalisierung und Prävention reduziert. Die Bereitschaft zur Selbstführung durch Bekenntnis und Verantwortungsübernahme wird mit (vorläufiger und begrenzter) $\mathrm{Zu}$ gehörigkeit belohnt. Diese reicht jedoch nicht aus und führt letztlich zu der Frage, wie (als) Muslim:innen (Markierte) angesichts ihrer prekären 
Anerkennung und des ihnen verweigerten Schutzes ihre Bedürfnisse nach Bildung, Sicherheit und Würde selbst organisieren können.

Die Diskussionsteilnehmer:innen sind sich der Anrufung bewusst, mit der sie dazu angehalten werden, ihren Beitrag zu Deradikalisierung und Prävention zu leisten. Sie wissen aus eigener Erfahrung, dass die Förderlandschaft auf sislamistischen Extremismus` fokussiert und finanzielle Anreize schafft mit dem Ziel, dass muslimische Gemeinden und Vereine die sicherheitspolitische Führung und Disziplinierung mittragen und sich selbst entsprechend führen. Sie kritisieren einerseits, auf die Rolle als >Sicherheitspartner im Kampf gegen Islamismus reduziert $\mathrm{zu}$ werden ${ }^{23}$, andererseits kann selbst in diesem Arbeitsfeld von einer Zusammenarbeit auf Augenhöhe keine Rede sein. Letztendlich interessiert ihr allgemeiner und gleichberechtigter Beitrag zur Gesellschaft, den sie als muslimische Mitbürger:innen leisten, nicht. Stattdessen steht ihre Loyalität als Deutsche zur Disposition und sie stehen als >Muslim:innen< unter Generalverdacht, sodass sie sich häufig damit konfrontiert sehen, mehr mit ihrer Verteidigung und Außenwahrnehmung zu beschäftigen als mit ihren community-internen Interessen und ihrem allgemeinen, gesellschaftlichen Engagement. Die >Partnerschaft<, die im Sicherheitsdiskurs zwischen Staat und muslimischen Communitys von ersterem eingefordert wird, ist unausgeglichen. Muslimische Communities machen die Erfahrung, dass Grundgesetz, Rechtsstaat, Demokratie, Sicherheit, Meinungs- und Religionsfreiheit $\mathrm{zu}$ ihren Ungunsten missgedeutet werden, also universalistische Werte partikular ausgelegt und Muslim:innen benachteiligt werden. Die Diskussionsteilnehmer:innen bemängeln die Intransparenz sicherheitspolitischer Apparate und Begriffsinstrumente. Das Konzept des `Gefährders` verunsichert und verletzt sie. Die Aufforderung, eigene Angebote nach sicherheitspolitischen Interessen auszurichten, um ihre erfolgreiche Integration und Demokratiefähigkeit zu beweisen, blendet aus, dass (als) Muslim:innen (Markierte) vielfältigen Diskriminierungen ausgesetzt sind, nicht zuletzt, indem ihre Integrations- und Demokratiefähigkeit kollektiv in Frage gestellt wird, ihre Anerkennung prekär 
bleibt und ihre Bedürfnisse - zum Beispiel nach Sicherheit und Gleichstellung - missachtet werden. In der Reflexion dessen stellt sich die Frage, wer eigentlich sie beschützt und ihre Sicherheit im Blick hat, die nicht zuletzt infolge des Sicherheitsdiskurses notwendig wird.

Im Zusammenspiel von Gouvernementalität und Bedrohungsszenario bringen sich (als) Muslim:innen (Markierte) als Subjekte hervor, die ihre Loyalität immer wieder neu unter Beweis stellen und ihre >Deradikalisierung als Lebens- und Community-Aufgabe zu erfüllen haben. Da die Selbstführung >Freiheit`voraussetzt ${ }^{24}$, kann im Zuge der Unterwerfung Handlungsmacht erlangt werden, sodass eigene muslimische Bedürfnisse in den politischen Diskurs eingeführt und die Anerkennung und Bekämpfung von antimuslimischem Rassismus als demokratisches Bürger:innenrecht eingefordert werden kann. Indem sie sich der Anrufung unterwerfen ${ }^{25}$ und Deradikalisierungs- und Präventionsarbeit leisten, gestalten sie den Diskursraum mit: Sie können Position beziehen und neben Erfolgen auch von der unangemessenen Diskursivierung sowie von politischen Fehlentscheidungen und Förderlücken berichten. Das Antworten ermöglicht eine Deutungsposition, von der aus der Staat selbst in die Verantwortung genommen wird.

Die Diskussionsteilnehmer:innen kritisieren, dass im Unterschied zu Präventions- und Deradikalisierungsprogrammen, die >Muslim:innen als (potentielle) Täter:innen in den Blick nehmen, Förderprogramme für Muslim:innen fehlen, und zwar solche, die antimuslimischen Rassismus thematisieren, Muslim:innen empowern oder, wie Gülçin D. es fordert, "ganz normale« Jugendarbeit anbieten. Sie rücken eigene Bedarfe ins Licht, die im Rahmen von Präventions- und Deradikalisierungsarbeit nicht zufriedenstellend bedient werden können. Sie fordern gleichberechtigte Teilhabe, auch bezüglich ihrer spezifischen Interessen, sodass >Freiheit $<$ und >Demokratie ihren universalistischen Ansprüchen gerecht werden. Die Relevanz und Bedeutung der Arbeit muslimischer Communities wird von ihnen selbst als Beitrag zur Ge-

\footnotetext{
24 Foucault 2017b; Sarasin 2005.

25 Butler 2006.
} 
samtgesellschaft betont und mit Hinweis darauf ihre uneingeschränkte Anerkennung und Gleichstellung eingefordert.

Die Argumentation gründet unter anderem auf allgemein gesellschaftlich geteilten und anerkannten Interessen (etwa Jugend- und Bildungsarbeit zu betreiben oder sich um die religiösen Bedarfe ihrer Gemeinde zu kümmern), auf ihrer faktischen Zugehörigkeit (hier geboren, aufgewachsen und lebend) und auf ihrem Anspruch, durch die deutsche Verfassung geschützt und gleichgestellt $\mathrm{zu}$ werden. In der Kritik, die in der Unterwerfung möglich wird, präsentieren sich unsere Gesprächspartner:innen als Bürger:innen, artikulieren ihre Interessen aus dieser Position und streben nach Handlungsmacht. Sie fordern ein, dass das Versprechen nach Teilhabe tatsächlich eingelöst wird und sie als gleichgestellte Bürger:innen anerkannt werden. Das setzt voraus, dass ihre Sorgen und Ängste ernst genommen, ihre Sicherheitsbedürfnisse nicht übergangen und ihre Forderungen nach Partizipation, Zugang zu Ressourcen und Investition in muslimische Strukturen stärker ins gesellschaftliche Blickfeld rücken. Muslimische Interessen treten im Sinne der gouvernementalen Vernunft ${ }^{26}$ als bürgerliche Interessen in Erscheinung, auf die der Staat reagieren muss, wenn er die (Selbst-)Führung nicht gefährden will. Gleichwohl vertrauen nicht alle Diskussionsteilnehmer:innen darauf, dass der Staat sie schützen wird. Es werden Möglichkeiten diskutiert, sich gegenseitig Schutz zu bieten. In gemeinsamen Visionen über eine community-nahe Solidarität und Zusammenarbeit mit anderen Muslim:innen tritt die Vorstellung eines gegen-hegemonialen Raumes in Erscheinung. Die Diskussionsteilnehmer:innen konzipieren einen heterotopen Raum $^{27}$, in dem nicht Misstrauen und Ent_Solidarisierung, sondern Gemeinschaft in Verschiedenheit gelebt wird. teressen, sondern erwächst aus dem »komplexen Spiel zwischen individuellen und kollektiven Interessen« (2017b: 73). 


\section{„Dicka, habt doch die Transparenz so!»}

Unsere Diskussionsteilnehmer:innen teilen die kollektive Erfahrung, dass ihre Loyalität als Deutsche zur Disposition steht. Sie stehen unter Generalverdacht und müssen auf Vorwürfe und Anschuldigungen reagieren. Sie unterwerfen sich dem Sicherheitsdiskurs, indem sie seine Sprache und Argumentation aufgreifen auf der Suche nach einem adäquaten Umgang damit. Eine Strategie besteht darin, Staat und Gesellschaft an ihre eigenen Maximen zu erinnern und ihnen zu spiegeln, dass sie es sind, deren Verhalten undemokratisch, unredlich und sozial unverträglich ist. Die Diskussionsteilnehmer:innen kehren also sowohl die Blickrichtung als auch den Vorwurf um.

In der dritten Gruppendiskussion diskutieren die Teilnehmer:innen über den `Gefährder«-Begriff. Leyla L. bemängelt, dass sie sich vom Sicherheitsdiskurs »verarscht « fühlt und fordert Transparenz: »Dicka, habt doch die Transparenz so!« Die kumpelhafte Anrede unterstreicht, dass sie sich auf derselben Seite wähnt. Sie spricht den Staat direkt an und durchbricht einerseits die Grenzziehung zwischen Muslim:innen und Deutschen und andererseits die im Sicherheitsdiskurs gegen Muslim:innen gerichtete einseitige Kontrollfunktion des Staates. Sie ruft ein Verhältnis zwischen Staat und Bürger:innen auf, das zwar arbeitsteilig, aber gleichberechtigt operiert: »So wenn wir, weißt du (.), wenn wir $\mathrm{n}$ gemeinsames Ziel haben, dann können wir doch zusammenarbeiten." Obwohl auch in dieser Gruppendiskussion die Solidarität zwischen Muslim:innen angesichts ihrer Diskriminierung verhandelt wird, werden in dieser Sequenz Muslim:innen, Staat und Dominanzgesellschaft in ein »wir« zusammengeführt, das ein "gemeinsames Ziel« verfolgt. Indem Leyla L. das »wir « so selbstverständlich formuliert, setzt sie die Gegenüberstellung eines Staates der Deutschen auf der einen Seite und Muslim:innen, deren Integration verhandelt wird und deren Zugehörigkeit zur Disposition steht, auf der anderen Seite, außer Kraft. ${ }^{28}$ In 
der Einladung zur Zusammenarbeit präsentiert Leyla L. Muslim:innen nicht nur als Bürger:innen, sondern lädt sie zu einer Kooperation auf Augenhöhe ein, um die parallel laufenden Bemühungen zusammen zu führen. Die derzeitige Situation ironisierend, formuliert sie ihre Aufforderung so, als hätte bislang einfach niemand daran gedacht, zu kooperieren (»dann können wir doch«). Gleich darauf übt sie offen Kritik am Staat, weil er Muslim:innen gegenüber nicht offen ist und ihnen nicht vertraut, so ihr Vorwurf: »Warum versteckst du was vor mir? Warum?« Sie lacht kurz auf und ergänzt »Weißt du so? Weil es ist halt so, is direkt so: Wen willst du hier verarschen. «Sie fühlt sich hintergangen. Ihr Redebeitrag wird von den anderen Diskussionsteilnehmer:innen rege und zustimmend kommentiert. Hassan M. sagt: Das "Problem« ist »nicht nur man=etwas versteckt, sondern man versteckt was, aber behauptet man dann, man sei transparent«. Intransparenz und Heuchelei sind klassische antimuslimische Topoi $^{29}$, die insbesondere im Sicherheitsdiskurs ihre Wirkung zeigen. Sie werden hier nun aufgegriffen und zurückgespiegelt. »Man« ist nicht nur intransparent, sondern gibt auch noch vor, transparent zu sein, mehr noch: »Man« wirft Muslim:innen vor, intransparent und heuchlerisch zu sein. Der Staat als wichtiger Akteur im Sicherheitsdiskurs wird demokratischen Ansprüchen offenbar nicht gerecht, wirft aber die eigenen Versäumnisse Muslim:innen vor, so die Argumentation. Die Diskussionsteilnehmer:innen hinterfragen die Machtstruktur und die doppelten Standards: Von Muslim:innen wird etwas erwartet, das ihnen gegenüber nicht eingehalten wird, dessen Missachtung ihnen aber wiederum vorgeworfen wird.

\section{"die Frage der Priorität und [die] Frage der Augenhöhe»}

Gülçin D. spricht aus der Perspektive eines Vereins, der Präventionsund Deradikalisierungsarbeit anbietet. Vor dem Hintergrund ihrer Erfahrungen in der konkreten Arbeit zweifelt sie an der Berechtigung des Bedrohungsszenarios: »Ich sehe das ja aus dem äh aus der Arbeit, die 
ich bei meinem Träger mache, dass eigentlich die Gefahr nicht so groß ist, dass wir andauernd radikalisierte Jugendliche haben.« Demgegenüber mangelt es an regulären Angeboten:

»[I] $\mathrm{m}$ Vorfeld braucht es mehr Aufklärungsarbeit unter Musli::men oder braucht es mehr politische Bildung, um die Menschen, (1) die $\mathrm{Mu}=$ Muslime, in dem stark zu machen, was sie gerade tun. Also seisind sie Schüler sind sie stä- zu stärken in ihrem Schülersein, wenn sie Student sind, sie zu stärken in ihrem Studium.«

Zwischen den politischen Interessen, die sich in Förderrichtlinien niederschlagen, und jenen muslimischer Communities besteht eine Diskrepanz: »[W]ir würden gerne da eigentlich andocken, ähm aber (1) das wird schwie::rig, weil die Töpfe, aus denen man Projekte beantragen kann (1), eben in diesem großen Topf untergebracht sind Sicherheit und Extremismusprävention.«

Darin drückt sich eine doppelte Diskriminierung aus. Die gewünschten Arbeitsschwerpunkte gehören zu den regulären Leistungen und Aufgaben der Kinder- und Jugendhilfe. Sie liegen in staatlicher Verantwortung und werden dem Subsidiaritätsprinzip zufolge von freien Trägern ausgeführt. Zusätzlich existieren Projektförderungen, die zeitlich befristet sind und auf spezifische, kurzfristige Problemlagen reagieren. Während Jugendarbeit und politische Bildung regelfinanziert sind, woran aber muslimische Träger erst zögerlich partizipieren können, fällt die Deradikalisierungsarbeit unter die Projektförderung. Gülçin D. schließt sich nun weder der verbreiteten Kritik an der Einschätzung an, dass politischer Radikalismus und Rassismus als kurzfristige Probleme behandelt werden, noch kritisiert sie die mangelnde Unterstützung an der Etablierung eines muslimischen Wohlfahrtsverbands. Sie begnügt sich vielmehr damit, Projektmittel (also eine zeitlich und thematisch begrenzte Förderung) für muslimische Jugend- und Bildungsarbeit $\mathrm{zu}$ fordern. Gülçin D.s Wunsch »Projekte $[\mathrm{zu}]$ beantragen« knüpft an ihrer Erfahrung an, als muslimischer Träger lediglich Gelder aus »diesem großen Topf [...] Sicherheit und Extremismusprävention« zu erhalten. Angesichts der 
anvisierten gesellschaftlichen Aufgaben, die sie übernehmen will, ist ihre Forderung bescheiden.

Sowohl die Bescheidenheit als auch die Unterwerfung stoßen auf Mohamed B.s Kritik. Muslim:innen wird nur zugestanden, im Rahmen von Projekten für die Lösung konkreter Probleme in die Verantwortung genommen zu werden: ")Es gibt ein Problem. Diese Muslime können das irgendwie auch ändern [...] aber sollen nur das bitte ändern und so wie es uns passt und nicht so, wie sie denken, dass es richtig wäre «. Aus dieser Sicht ist diese Praxis aus verschiedenen Gründen problematisch: Muslim:innen haben keinen Anteil daran, das Problem zu definieren oder ein Handlungskonzept zu entwerfen. Sie sind lediglich als Ausführende vorgesehen. Die sicherheitspolitische Rahmung des Handlungsbedarfs hält Muslim:innen auch perspektivisch aus der Verantwortung heraus, reguläre Jugendarbeit ist nicht vorgesehen. Insofern argumentiert Mohamed B. sowohl mit als auch gegen Gülçin D.s Strategie. Auch er hält reguläre Angebote für sinnvoll, zweifelt aber daran, dass Muslim:innen mehr gewährt wird als »ein Problem [...] irgendwie [zu] ändern«. Ihm stellt sich »die Frage der Priorität und [die] Frage der Augenhöhe«. Muslimischen Verbänden wird im Rahmen des Sicherheitsdiskurses lediglich eine nachgeordnete Akteursposition zugestanden: "Man betrachtet die muslimischen Gemeinden nicht als ebenbürtig", sie sollen so arbeiten, "wie es uns passt und nicht so, wie sie denken, dass es richtig wäre«. Die Perspektiven, Erfahrungen und Wissensbestände von Muslim:innen finden keine Berücksichtigung, vielmehr werden Aspekte davon für sicherheitspolitische Interessen instrumentalisiert.

Gülçin D. ist um einen pragmatischen Ansatz bemüht. Sie stimmt Mohamed B.s Analyse und Kritik zu (»Das stimmt, da hast du Recht, aber nur das-«) und widerspricht gleichzeitig seiner Strategie. Sie teilt seine Kritik an der Übertragung sicherheitspolitischer Aufgaben an muslimische Verbände, da ihre Kompetenzen nicht in diesem Bereich liegen: »Plötzlich müssen sie irgendwelche Projekte stemmen, mit denen sie vielleicht gar nichts $\mathrm{zu}$ tu:n haben.« Gleichzeitig wird ihnen verwehrt, sich in einer Weise gesellschaftlich einzubringen, die ihren Fähigkeiten entspricht: »[U]nd sie hätten vielleicht andre 
Sachen, die sie bisher gut gemacht haben. Wenn man das fördert, vielleicht viel bessere Arbeit auf die Beine bringen können«. Sie schwächt ihre Kritik $a b$, indem sie gleich dreimal ihre eigene Aussage in Frage stellt (»vielleicht«). Weder sind muslimische Akteur:innen eindeutig nicht in der Lage, sicherheitspolitische Verantwortung zu übernehmen, noch kann sie eindeutig bestätigen, dass sie anderes besser können und dass dieses andere auch fruchtet. Die größtenteils ehrenamtliche Arbeit der Verbände bedarf der Professionalisierung. Deren Förderung wird weitgehend erfolglos eingefordert. Stattdessen werden von außen andere Aufgaben an die Verbände herangetragen und gefördert. Die ihnen zugedachte Rolle anzunehmen, beruht nicht nur auf strategischen Überlegungen, sondern ist auch der materiellen Situation der Verbände geschuldet. Über Deradikalisierungsprojekte können Verbandsstrukturen professionalisiert werden, allerdings nur einen Projektzeitraum lang. Die Grenze zwischen Selbstführung und Disziplinierung verschwimmt.

Gülçin D. argumentiert, dass muslimische Jugendarbeit den Effekt hat, gesellschaftlich unerwünschtes Verhalten zu reduzieren: »Also es gibt so viele Sachen, die man machen müsste, damit es gar nicht erst zur Radikalisierung kommt oder zu dem Geda:nken einer Radikalisierung. So viel Ju::gendarbeit aus muslimischer Perspektive. « Sie wirbt in der Gruppendiskussion mit muslimischen Teilnehmer:innen für eine sicherheitspolitische Begründung von Jugendarbeit und versucht, ihr pragmatisches Vorgehen gegenüber der Politik zu normalisieren. Mohamed B. deutet Gülçin D.s Ziel, muslimische Jugendarbeit zu professionalisieren, als gemeinsames Anliegen von Muslim:innen. Im Unterschied dazu geht es »der Politik« seiner Ansicht nach um etwas anderes: »Anschläge zu verhindern, einzuschränken oder geringer zu haben«, also restriktive Maßnahmen durchzusetzen. Demgegenüber wollen muslimische Verbände junge Leute stärken, so Mohamed B. Der nicht näher spezifizierte Begriff im Plural »Anschläge« deutet auf die Gewalt hin, die von >Muslim:innen ausgeübt wird. Nicht gemeint sind hier Anschläge, die gegen Muslim:innen gerichtet sind. Insofern zielen die von »der Politik« eingeführten Maßnahmen im Zusammenhang mit »Anschlägen« nicht auf die Stärkung von Muslim:innen. Vielmehr 
steht ihre Thematisierung als Täter:innen im Zentrum. Mohamed B. bescheinigt »der Politik« zudem Hilflosigkeit in der Konzeption sinnvoller Maßnahmen: »[M]ach mal irgendwas, aber bewegt euch nicht $\mathrm{zu}$ viel.«Aus seiner Perspektive mangelt es der Politik an klaren Konzepten, lediglich das Ausmaß an Handlungsfähigkeit wird begrenzt. Die Anrufung von muslimischen Verbänden als Akteure nimmt sie einerseits in die Verantwortung, gesteht ihnen aber nicht zu viel davon zu: » [S]o wie ihr noch in der Ecke seid, ist es ganz gut«", sagt Mohamed B. sarkastisch; Muslim:innen sollen »nicht zu sehr in Erscheinung treten«.

Die Diskussionsteilnehmer:innen sind sich nicht einig, ob ihnen eine unterwerfende Antwort auf die Anrufung muslimischer Verbände als >Sicherheitspartner $<$ perspektivisch ermöglicht, als reguläre Partner Verantwortung übernehmen zu können oder aber sie instrumentalisiert und »in der Ecke« hält. Einig sind sie sich dagegen darin, dass sie Verantwortung übernehmen wollen, und zwar als muslimischer Teil der Gesellschaft. Hierzu muss die Politik muslimischen Akteuren auf Augenhöhe begegnen, sodass andere Prioritäten möglich werden.

\section{"eigentlich brauchen wir was ganz anderes, wir brauchen eigentlich eine Stärkung der Community«}

Viola E. antwortet ebenfalls auf den antimuslimischen Sicherheitsdiskurs und hält muslimische Jugendarbeit für eine gute Möglichkeit zur Prävention. Sie kehrt allerdings die Argumentation des Sicherheitsdiskurses um. Nicht »zu viel Islam« führt aus ihrer Sicht zu »Extremismus« und »Terrorismus«, sondern »zu wenig«: »In der Realität ist es ja tatsächlich so:, dass nicht ä::h zu viel Isla:m äh dieses Problem erzeugt hat, sondern ä zu wenig oder zu wenig Wissen über den Islam. « «xtremisten « haben »skurrile Vorstellungen « und das rührt daher, »dass man eben überhaupt nicht mehr verwurzelt ist und dass (1) ganz grundlegende Dinge, die die Religion ja auch ausmachen [...], dass das überhaupt nicht mehr klar ist«. Viola E. diskutiert im Unterschied zu Gülçin D. nicht die Frage, wie groß das Radikalisierungsproblem ist. Einig sind sie und die anderen Diskussionsteilnehmer:innen sich jedoch in der Theoretisierung des Problems und der Konzipierung von Handlungs- 
konzepten. Viola E. knüpft an den Sicherheitsdiskurs in einer Weise an, die den Staat darin bestätigt, dass etwas geschehen muss. Sie sieht sowohl den Staat als auch muslimische Verbände in der Verantwortung zu handeln. Sie führt aber andere Gründe für das Problem an, insofern weicht auch ihr Vorschlag ab: »[E]igentlich brauchen wir was ganz anderes, wir brauchen eigentlich eine Stärkung der Community. Wenn wir äh, wenn Moscheegemeinden äh sich äh Räume leisten könnten (1) und ganz normal ihre Arbeit machen (1) könnten«. Gülçin D. und Mohamed B. stimmen Viola E. zu, sie unterbrechen sie bestätigend und führen ihre Sätze analog zu Ende.

Das hier in Anschlag gebrachte Wissen widerspricht dem sicherheitspolitischen: Die muslimische Deutung des Problems und seiner Lösung weist das hegemoniale Wissen zurück. Die muslimische Sicht auf ihre Religion und die Einschätzung der Akteur:innen bezüglich ihres Potentials zur Extremismusprävention steht der Außenwahrnehmung gegenüber. Dass sie dennoch auf ihre Anrufung als $>\mathrm{Si}$ cherheitspartner:innen antworten und Verantwortung übernehmen, gründet auf ihrem Selbstverständnis, dass auch sie sich als geeignete Ansprechpartner:innen für das Thema erachten, wenn auch aus anderen Gründen. Indem sie in den Sicherheitsdiskurs eintreten, eröffnet sich ihnen die Möglichkeit, das hegemoniale Wissen über den Islam und über Radikalisierungsprozesse zu verschieben, zumindest aber zu pluralisieren. Das hegemoniale Wissen über Islam und Radikalisierung präsentiert sich als Wahrheit, ist aber partikular und situiert. Es bildet nicht die Erfahrungen und das Wissen der muslimischen Diskussionsteilnehmer:innen ab. Auch ihr Wissen ist situiert. Im Unterschied zum hegemonialen Wissen kennen Marginalisierte aber sowohl die eigenen als auch die hegemonialen Wissensbestände und reflektieren die Begrenztheit und Standortgebundenheit des jeweiligen kommunikativen Wissens. Den Diskussionsteilnehmer:innen ist die Kontingenz der kommunikativen Wissensbestände vertraut. Das Ver_Antworten bietet ihnen die Gelegenheit, das Präventionsparadigma um eigene Bedürfnisse zu erweitern und für ihre Sichtweise auf den Islam sowie auf Radikalisierungsprozesse in der Dominanzgesellschaft Gehör zu finden. In der Präventions- und Deradikalisierungsarbeit Verant- 
wortung zu übernehmen, bietet ihnen zudem die Möglichkeit, die konkrete Arbeit nach ihrem eigenen Verständnis und den Bedürfnissen der "ganz normalen Jugendlichen« zu gestalten.

Die Hoffnung auf Änderung des Diskurses ist jedoch brüchig. Schließlich bestätigen auch jene Diskussionsteilnehmer:innen, die sich auf die Sicherheitspartnerschaft einlassen, dass sie nicht "auf Augenhöhe« stattfindet und ihre »Prioritäten« andere sind. Die kurzfristige Projektfinanzierung erlaubt es nicht, grundständige Aufgaben $\mathrm{zu}$ übernehmen und sich zu professionalisieren, selbst Räume fehlen, um kontinuierlich und nachhaltig arbeiten zu können. In der zweiten Gruppendiskussion konfrontiert Samir H. die Aufforderung an Muslim:innen, sich an der "Deradikalisierung « $\mathrm{zu}$ beteiligen mit der fehlenden Bereitschaft des Staates, im Gegenzug die "Betroffene[n] des eigenen Sicherheitsdiskurs « zu unterstützen. Er betont die »krasse Diskrepanz [...], Sicherheitsdiskurs hat ne Auswirkung auf unsere Lebensrealität, eben antimuslimischer Rassismus, Ausschlussmechanismen. Sowohl individuell als Muslime, als auch als Organisation, hat ein=ein äh (.) ä:h realen Effekt auf unsere Lebensrealität«. In seinem Bundesland gibt es bis dahin »kein einziges Programm gegen antimuslimischen Rassismus. ${ }^{\circ}(2)$ « Indem er den antimuslimischen Rassismus in einen Zusammenhang mit dem Sicherheitsdiskurs bringt, stellt er die Dringlichkeit von Projekten gegen antimuslimischen Rassismus heraus. Neben der üblichen Jugend- und Bildungsarbeit, die muslimische Träger als reguläre Aufgabe wie alle anderen »Teile der Gesellschaft« leisten wollen, sind Projekte gegen antimuslimischen Rassismus zumindest als Folge des Sicherheitsdiskurses wie Projekte zu Prävention und Deradikalisierung zu behandeln. Insofern nehmen die Diskussionsteilnehmer:innen ihrerseits den Staat in die Verantwortung, für ihre Bildung und Entwicklung sowie für ihre Sicherheit und ihr Empowerment zu sorgen. Sie kritisieren auf unterschiedliche Weise, dass Muslim:innen einseitig in die Verantwortung genommen werden, indem sie als (potentielle) Gefahr angerufen werden und von ihnen disziplinierende und gouvernementale Kooperation erwartet wird, ohne dass ihren eigenen Partizipationsansprüchen und 
Sicherheitsbedürfnissen als gleichberechtigte Bürger:innen und damit demokratischen Prinzipien entsprochen wird.

\section{»irgendwie müssen wir füreinander Schutzschilder sein«}

Die Auswirkungen des Sicherheitsdiskurses auf (als) Muslim:innen (Markierte) sind gravierend. Der Sicherheitsdiskurs als Dimension des antimuslimischen Rassismus hat nicht nur Folgen auf die Förderung, die Disziplinierung und das gesellschaftliche Ansehen von Personen und Gruppierungen, sondern dringt in die Körper und in die Psyche von (als) Muslim:innen (Markierten) ein. Leyla L. berichtet in der dritten Gruppendiskussion, dass es einen Unterschied macht, mit wem sie sich im öffentlichen Raum bewegt: Wenn sie mit Personen unterwegs ist, die als asiatisch wahrgenommen werden, dann werden sie als Tourist:innen adressiert und auf Englisch angesprochen: »[W]ir sind keine Gefahr quasi, wir sind nur ne Kaufkraft quasi.« Das kann amüsant sein. Im Unterschied dazu belastet es sie sehr, wenn sie sich mit männlichen, insbesondere jüngeren, als muslimisch markierten Personen in der Öffentlichkeit bewegt:

»[W]enn ich aber mit meinem Vater (.) aber eigentlich hauptsächlich mit meinem Bruder unterwegs bin [...] uns beiden ist es bewusst [...]

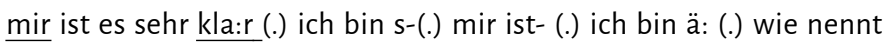
man das (.) ä: (.) ich bin wach, ich bin wach, ich guck, jede Sekunde, könnte jetzt was kommen.«

Die ständige Angst und die Vorsicht vor möglichen verbalen oder körperlichen Verletzungen führt dazu, dass sie sich ihrer Markierung als Muslimin deutlicher bewusst ist und folglich ihre muslimischen Bezüge auch ihr selbst stärker ins Bewusstsein rücken. Die Alltäglichkeit solcher Situationen wirkt identitätsstiftend, sodass »dann meine Identität auch stärker muslimisch ist mit meinem Bruder zusammen, also wenn wir zusammen unterwegs sind und die Sache ist (.) mittlerweile hat sich das so normalisiert«. Identität beschreibt Leyla L. nicht als Wesenseigenschaft, sondern als Antwort, als Relation zwischen ihr und der Außenwelt. Sie führt ihre Haut als Medium zwischen Innen und Außen 
an: Im Ausland, »wo ich nicht als (.) muslimisch rassifiziert werde [...] wo ich einfach nur als Touri oder so gesehen werde (.) es lö:st sich eine Haut von mir, es entsteht eine Leichtigkeit«. Hassan M. kennt diesen Unterschied auch, und zwar zwischen seinem früheren Leben und seiner aktuellen Situation. ${ }^{30}$ Als Person, die muslimisch, männlich, jung wahrgenommen wird, kann er jedoch "nicht ausschalten, weil ich erlebe das tagtäglich, am eigenen Leib«. Er kennt »diesen Luxus« nicht mehr, sich »ohne nachzudenken (.) ähm was sagen die Leute jetzt« in der Öffentlichkeit zu bewegen. Auch Tasnim J. setzt der Sicherheitsdiskurs zu:

»[I]ch hab soviel (.) äh soviel Unrecht äh gese:hen und (.) ähm ich hab so n starkes Cefühl von Ungerechtigkeit, die immer und immer und immer wieder passiert ((hustet)) und ä:hm (1) wie ä: ja es treibt dich in so'ne Ecke [...] ich glaube wir tra:gen soviel von diesem psychischen (.) Fuck-up und dieser=dieser (.) also es ist für mich (.) wie (.) also es ist wie Vergewaltigung einfach (.) psychisch, die ga:nze Zeit.»

Die »Normalität, dass wi:r (.) ähm ä als gefährlich wahrgenommen werden«, wird »internalisiert « und wirkt sich darauf aus, »wie man seinen eigenen Körper sozusagen als gefährlich wahrnimmt«.

Die langen Ausführungen aller Diskussionsteilnehmer:innen werden von den anderen nicht unterbrochen, sie hören konzentriert $\mathrm{zu}$. Bei der Suche nach dem richtigen Wort für die psychischen Folgen der Normalisierung von Rassismuserfahrungen helfen die Diskussionsteilnehmer:innen aus: »[D]u isolierst dich oder du vernichtest dich oder du findest Wege und Strategien, um irgendwie weiter zu machen«, man versucht, die »Gewalt [zu] sublimieren«, man wird »stumpf [...] gegenüber diesen ganzen Bildern und den ganzen Diskussionen«, »akzeptiert « sie, ist "gelangweilt«, »distanziert« sich, aber bei erneuten »Mikroaggressionen«»kommt es wieder hoch«. Die Diskussionsteilnehmer:innen fühlen sich ausgeliefert und ungeschützt. Besonders hilflos macht es sie, wenn ihre Kinder und andere jüngere Menschen von Rassismus getroffen werden. Asmah N. stimmt in die Diskussion ein: 
»Ich hab schon Angst (.) ä::hm (1) ich ä: ich hab kleine Jungs [...] es gibt so Sachen, mit denen kann ich umgehen (.) oder ich hab die für mich schon einmal durch [...] es ist nicht für immer und ewig stabil und so ( ) ja, es nervt [...], aber jetzt hab ich- jetzt gibt's ne kleine Ceneration (.) von jung, männlich, arabisch, musli:misch, das ist jetzt da so (.), also meine=meine Angst richtet sich so'n bisschen auf die, deswegen ich bin sehr, sehr besorgt, ähm (.) und ich merk auf der einen Seite, wie ich mich auch gerade als Frau so'n bisschen als ä::h menschliches Schutzschild fühle ((bestätigendes Gemurmel)), dass es wichtig ist, Frauen müssen da sein, wo Männer sind [...] ich mach da überhaupt keine Abstriche an feministischen Fragen, a:ber (.) ich mach mir richtig So:rgen um die Männer (.) um meinen Mann, um meine K- nicht nur meine Kinder, sondern um alle jungen Männer da draußen, also alle Braunen jungen Männer [...] irgendwie müssen wir füreinander Schutzschilder sein, ganz sta:rk.«

Als Verbandsvertreter:innen entwickeln die Diskussionsteilnehmer:innen Ideen zum professionellen Umgang mit dem Sicherheitsdiskurs, mit der Adressierung als >Sicherheitspartner und mit der Ausgestaltung muslimischer Jugend- und Bildungsarbeit. In dieser Sequenz aber wird der Austausch sehr persönlich und intim, Aktivismus und Engagement weichen der Exploration und Reflexion ihrer Gefühle. ${ }^{31}$ Die Antworten auf den Sicherheitsdiskurs sind nicht länger an den Staat und die Gesamtgesellschaft gerichtet, sondern an sich selbst. Die Frage danach, wie besonders vulnerable Muslim:innen und als solche Markierte vor allzu großen Verletzungen beschützt werden können, nimmt die gegenseitige Verantwortung in den Blick. Es wird deutlich, dass intersektionale Aspekte nicht nur in der Einschätzung besonders gefährdeter Personengruppen, sondern auch in der Erwartung an Schutz eine zentrale Rolle spielen.

Auf Asmah N.s Plädoyer, »füreinander Schutzschild [zu] sein«, entsteht eine Diskussion zu der Frage, wie angesichts der intersektio-

31 Die emotionale Ebene wird im Kapitel Selbst_Positionierungen im Abschnitt Schützende Distanzierungen und in Keskinkılıç 2021 i.E. diskutiert. 
nal-muslimischen Erfahrungen Verantwortung übernommen werden kann. Asmah N. hat das starke Gefühl, sich als Frau insbesondere schützend vor jüngere Männer stellen zu müssen. Sie selbst fühlt sich geschützt, wenn jüdische Personen ihr als Palästinenserin beistehen. Leyla L. wirft ein, dass sie als Hijabi regelmäßig von Trans* of color in Schutz genommen wird. Sie vermisst dagegen, dass muslimische cis-Männer weibliche und queere Muslim:innen verteidigen, wenn sie diskriminiert werden. Hassan M. fühlt sich als einziger Mann im Raum angesprochen und gibt zu verstehen, dass er befürchtet, dass seine Fürsprache als muslimisch markierter Mann für muslimische Frauen und Queers instrumentalisiert wird und den antimuslimischen Rassismus affirmiert. Er ruft das hegemoniale Wissen auf, dass die Markierung des muslimischen Mannes als aggressiver Täter mit der Markierung der muslimischen Frau als sein passives Opfer korrespondiert. Hassan M.s Zurückhaltung rührt daher, dass er den hegemonialen Diskurs nicht bestätigen und auch nicht als Täter adressiert werden möchte. Asmah N.s Szenario, wonach sich Frauen schützend vor Männer stellen, ist insofern ambivalent auf den Islamdiskurs bezogen, als dass in ihrem Szenario Frauen als stark erscheinen, sodass sie in der Anordnung von Schutzbedürftigen und Schützenden den orientalistischen Opferdiskurs durchkreuzt. Gleichzeitig reflektiert Asmah N., dass die geschilderte Konstellation missgedeutet werden kann, nämlich als antifeministische Geste. Sie betont deswegen, dass sie diesbezüglich »keine Abstriche« macht. Sie bestätigt Hassan M. in seiner Zurückhaltung und kehrt das feministische Argument um: »Leute, die schwächer sind als ich, die können nicht mein Schutzschild sein.« Die Schwäche muslimisch markierter Männer resultiert aus ihrer Diskursivierung als Gefahr. Asmah N. weist die antimuslimische Diskursivierung des muslimischen Geschlechterverhältnisses durch eine Anordnung zurück, die sie entgegen der üblichen religiösen und kulturellen Rahmung gesellschaftlich akzentuiert. Auch Leyla L. bezieht in ihren Wunsch an Community-Mitglieder den gesamtgesellschaftlichen Kontext ein, sowohl in ihrer Aufforderung, in konkreten Diskriminierungssituationen beschützt $\mathrm{zu}$ werden, als auch in der Art und Weise des Antwortens. Sie wünscht sich Unterstützung von 
anderen Muslim:innen, um denjenigen, die diskriminieren, deutlich zu zeigen: »[W]ir sehen euch.«

Die nach Innen gerichtete emotionale Öffnung kann angesichts des gesellschaftlichen Kontextes nicht lange aufrecht erhalten werden. Dieser rahmt und durchdringt die Schilderungen auch dann, wenn die Antworten und Aufforderungen einander gelten. Der hegemoniale Diskurs begründet den Wunsch nach »menschlichem Schutzschild« und muss in seiner Wirkung auf die Gesellschaft berücksichtigt werden, weil dieser wiederum auf Muslim:innen zurückwirkt. Die möglichen Effekte vorwegnehmend schließt die Aushandlung der gegenseitigen Verantwortungsübernahme die Kritik am antimuslimischen Rassismus ein. Mit Blick auf mögliche Konsequenzen stellt sich die Frage danach, wie Schutz gewährt werden kann und gleichzeitig Machtverhältnisse berücksichtigt, vielleicht sogar umgekehrt werden können. Leyla L. möchte nicht länger beobachtet, kommentiert, reglementiert und sanktioniert werden, vielmehr kehrt sie zumindest das Blickregime um: »Wir sehen euch «, in der Hoffnung, dass dies Wirkung zeigt. ${ }^{32}$

\section{„Wo sind die ganzen Princes?»}

In der geschilderten Sequenz resigniert Fatouma K. und stellt fest, »dass es keine Schutzschilder für uns gibt, deswegen muss man auch fragen, Sicherheit für wen? « Hassan M. ist noch nicht bereit, aufzugeben. Er unterscheidet verschiedene Strategien des Umgangs mit dem Sicherheitsdiskurs und dem antimuslimischen Rassismus im Allgemeinen und ruft Umgangsformen in Erinnerung, die nach innen statt nach außen gerichtet sind. Jenseits der medialen und politischen Verwertungslogik zielen diese auf die Stärkung der Community im Innern. In allen Gruppendiskussionen wird dieser Gedanke im Wunsch nach eigenen, sicheren, stärkenden Räumen deutlich, in denen Themen bearbeitet werden können, die Muslim:innen selbst für relevant chert wird, wenn rassifizierte Frauen weiße Männer sehen, diesen aber der Blick auf sie verwehrt ist. 
erachten und die im Zuge der Sicherheitslogik des Bedrohungsszenarios zurückgedrängt werden, wie etwa eine muslimisch akzentuierte Jugendarbeit oder Maßnahmen, die infolge von Rassismus notwendig werden, wie Schutz, Empowerment und Heilung.

Im Anschluss an die sehr intime und konzentrierte Sequenz "füreinander Schutzschilder sein « entsteht in der dritten Gruppendiskussion ein lebhafter Austausch über Visionen von Community-Zentren und geschützten Räumen. Im Vergleich zu ihren Erfahrungen in Großbritannien vermisst Tasnim J. in Deutschland eine CommunityInfrastruktur. Andere ergänzen, dass es selbst in Kunst und Comedy in Deutschland nicht jene künstlerische Freiheit für Muslim:innen gibt, die, als Gegenmodell zu den bürokratisierten Strukturen der Zivilgesellschaft, Ausdrucksmöglichkeiten bieten könnten. Leyla L., die als Künstlerin tätig ist, widerspricht und berichtet, dass weiße Strukturen nicht vor künstlerischen Projekten halt machen. Aufgrund der finanziellen Abhängigkeit freiberuflicher Kunst- und Kulturschaffender wird auch hier an den Sicherheitsdiskurs angeknüpft. "Der deutsche Staat hat alles im Griff, was die Zivilgesellschaft betrifft«, resümiert Tasnim J. und lenkt damit das Thema zurück zur Führung durch die Kontrolle finanzieller Ressourcen. Angesichts des staatlich reglementierten Zugangs zu materiellen Ressourcen und der eingeschränkten Möglichkeiten, eigene Themensetzungen zu finanzieren, fragt Tasnim J.: »[W]o sind unsere privaten Funders?«, woraufhin Leyla L. - den Orient- und Islamdiskurs karikierend - sekundiert: »Wo sind die ganzen Princes? « Leyla L. greift implizit auf das Stereotyp des Öl-Scheichs zurück, das vom orientalistischen Märchenprinzen hin zur weltbeherrschenden Macht mutiert ist und nun, so der antimuslimische Verschwörungsmythos, die Medien-, Politik-, Justiz- und Bildungslandschaft steuert. Das Stereotyp ist allen bekannt, das Auflachen kommentiert die Absurdität der Vorstellung, dass saudiarabische Milliardäre rassifizierte und marginalisierte Minderheiten in Europa fördern könnten.

Asmah N. greift die Frage nach alternativen Handlungsmöglichkeiten auf und meint, dass Strategien sich manchmal ändern müssen. Sie unterscheidet zwischen solchen, die innerhalb des hegemonialen Diskurses dessen Verschiebung anstreben und anderen, die von außen In- 
novationen anstoßen. Das erinnert an die zweite Gruppendiskussion, in der sich die Unterscheidung nach inneren Auseinandersetzungen und äußeren Neugründungen aber auf die muslimische Community bezog. Asmah N. hält es manchmal für angemessener, ihre Bedürfnisse nicht laut $\mathrm{zu}$ artikulieren und wird dabei von Leyla L. unterstützt, die bei sich bleiben, eigene Interessen und Ziele verfolgen und sich nicht von Rassismen und den Anliegen der Anderen ablenken lassen will. Sie kann auf diese Weise anderen Muslim:innen Vorbild sein, da es ihrer Wahrnehmung nach »viele kaputte Menschen in den Communities« gibt, denen sie ihre Aufmerksamkeit zukommen lassen möchte. Auch Asmah N. hat jene Muslim:innen im Blick, denen mangels Bildungsprivilegien weniger Ressourcen zum Umgang mit den Anschuldigungen und Ausschlüssen zur Verfügung stehen.

In allen Gruppendiskussionen zeigen sich Bereiche, in denen Muslim:innen als Muslim:innen bereit sind, Verantwortung zu übernehmen, mehr noch, diese Möglichkeit der Verantwortungsübernahme füreinander und auch für die Gesamtgesellschaft einfordern. Darunter fallen muslimische Jugendarbeit und religiöse Unterweisung, Auseinandersetzungen mit dem antimuslimischen Rassismus und muslimische Zentren als Orte der Begegnung und Stärkung untereinander. Um dies zu erreichen, appellieren sie an die Politik, demokratische Prinzipien einzuhalten, mit muslimischen Akteur:innen auf Augenhöhe zu kooperieren, sie als genuinen Teil der Gesellschaft mit gleichen Rechten und Ressourcen auszustatten und ihnen eigene Räume zuzugestehen. An sich selbst und aneinander richten die Diskussionsteilnehmer:innen die Erwartung, in Anerkennung ihrer Differenzen solidarisch miteinander im Gespräch zu bleiben und sich gegenseitig zu unterstützen. 


\section{Selbst_Positionierungen}

In zehn Einzelinterviews baten wir Muslim:innen, die sich entweder selbst als solche bezeichnen oder als solche adressiert werden, uns zu schildern, wie sie den Sicherheitsdiskurs wahrnehmen, welche Auswirkungen er auf sie hat und wie sie damit umgehen. Uns interessierte, wie sie sich angesichts ihrer Adressierung als >bedrohlich (und) fremd selbst positionieren. Um ein möglichst breites Feld an Deutungen und Praktiken $\mathrm{zu}$ erhalten, versuchten wir, möglichst diverse Gesprächspartner:innen für Interviews zu gewinnen. Zur Auswertung nutzten wir die Objektive Hermeneutik als Werkzeug und passten sie unserer Fragestellung sowie unseren erkenntnistheoretischen und forschungsethischen Positionen an. An den Einzelinterviews nahmen die folgenden Personen (Pseudonyme) teil: Adem O., Ibtissam P., Khadija R., Rabea S., Latifa T., Can U., Jasmin V., Martin W., Ussama X. und Merve Y.

Die folgenden drei Abschnitte fassen unsere Forschungsergebnisse aus den Einzelinterviews zusammen. Die Fokussierung auf die genannten Aspekte »Schützende Distanzierungen «, »Performative Interventionen« und »Globalhistorische Verflechtungen « entwickelten wir induktiv aus dem Material. Der Datenerhebung und -auswertung gingen theoretische, ethische und methodologische Auseinandersetzungen voran, die den Forschungsprozess begleiteten und in Form neuer Erkenntnisse und Fragen an Theorien sowie Forschungsmethoden und -ethik darüber hinausragen. 


\section{Schützende Distanzierungen}

Das Bedrohungsszenario nimmt Muslim:innen als Sicherheitsrisiko in den Blick, während ihre eigenen Sicherheitsbedürfnisse nicht berücksichtigt werden. Es werden sicherheitspolitische Themen an sie herangetragen, sie werden aufgefordert, sich damit zu beschäftigen und dazu zu positionieren. Auf ihre Adressierung als >muslimische Bedrohung ‘ntworten unsere Gesprächspartner:innen unterschiedlich. Sie setzen - mehr oder weniger bewusst - verschiedene Strategien ein, um verletzenden Erfahrungen präventiv auszuweichen oder sich davon zu erholen. ${ }^{1}$ Sie gehen intellektuell auf Distanz, indem sie sich mit anderen Themen beschäftigen, sie gehen körperlich auf Distanz und wechseln die Arbeitsstelle oder den Wohnort und sie gehen emotional auf Distanz, indem sie ihre Erfahrungen verdrängen oder die überwältigenden Gefühle, die sie auslösen, zu bändigen versuchen. Sie kehren das Distanzierungsgebot gegenüber dem >islamistischen Terrorismus und >bösen $<$ Muslim:innen um, indem sie ihrerseits in Distanz zum Bedrohungsszenario gehen. Sie kritisieren, dass ihre Erfahrungen, ihre Wahrnehmungen und ihre Bedürfnisse kaum Eingang in den Sicherheitsdiskurs finden, obwohl ihre Sicherheit unter anderem durch die Omnipräsenz und durch die Gewalt des Bedrohungsszenarios gefährdet ist. Sie erleben Rassismus als intellektuelle, physische und emotionale Herausforderung und versuchen ihr auf diesen Ebenen zu begegnen. Der Einschreibung in ihre Körper und Psyche versuchen sie durch befristete Migration, berufliche Entscheidungen und emotionale Strategien zu begegnen. Sie analysieren Rassismus in seiner Bedeutung für ihr Befinden und suchen nach Wegen, sich davon zu distanzieren, um sich in Sicherheit zu bringen.

1 Zum emotionalen Umgang mit Rassismuserfahrungen: Ahmed 2018; Mecheril 1994. 


\section{»0kay ich will mich damit nicht mehr befassen«}

Auf die Einstiegsfrage, wie Latifa T. die Sicherheitsdebatten wahrnimmt und beurteilt, in denen Muslim:innen als Sicherheitsbedrohung dargestellt werden, bemerkt sie, dass diese ihr Leben »total« strukturieren. Ihre Rassifizierung stellt für sie einen »Zugang« zum Thema dar, dem sie sich nicht entziehen kann. Dem Alltagsrassismus, dem sie tagtäglich ausgesetzt ist, stellt sie allerdings selbstermächtigend ihre eigene Entscheidung entgegen: Sie will sich »damit nicht mehr befassen«. Es wirkt zunächst so, als stehe es ihr frei, sich zu entscheiden, ob sie eine Rassismuserfahrung als solche zur Kenntnis nehmen will, als handelte es sich um irgendein Thema, das sie aufgreifen kann oder auch nicht. Latifa T. ist sich jedoch darüber im Klaren, dass sie sich als rassifizierte Person dieser Erfahrung nicht entziehen kann, weil sich ihr Rassismus nicht als selbstgewähltes Thema stellt, sondern ihren Alltag in einer antimuslimisch-rassistisch strukturierten Normalität durchzieht. Sie wünscht sich, dass es anders wäre und weiß doch, dass sie im Unterschied zu weißen Personen keine Wahl hat zu entscheiden, ob sie sich mit Rassismus »befassen« will. Sie weiß auch, dass ihre Beschäftigung damit positioniert ist: "Äh ich glaub, wenn man davon direkt betroffen ist, dann hat man nochmal $\mathrm{n}$ ganz anderen Zugang äh dazu.«Rassismus ruft rassifizierte Menschen permanent dazu auf, sich mit dieser Erfahrung zu beschäftigen und hindert sie daran, andere Dinge zu tun. Sich zu entscheiden, andere Dinge zu tun und sich nicht von Rassismus aufhalten zu lassen, ist eine mögliche Umgangsweise mit Rassismus. Die Person entzieht sich der Antwort und konzentriert sich stattdessen auf andere Dinge. Sie entscheidet sich dazu, die Antwort schuldig zu bleiben, zu schweigen, auszuweichen oder sich abzuwenden, und damit doch, wenn auch auf verweigernde Weise, zu antworten.

Latifa T. arbeitet mit einer weißen Partnerin in einem eigenen Laden. Sie hat sich einen Raum geschaffen, in dem sie sich mit anderen Themen als mit Rassismus beschäftigen kann. Sie wendet sich Dingen $\mathrm{zu}$, die sie und ihre Community betreffen, und ist sich währenddessen gewiss, dass ihre weiße Partnerin die Verantwortung für Rassismus 
übernimmt, sich ihm stellt, sich informiert und engagiert - sie ist ohnehin »viel engagierter und informierter « als Latifa T. selbst. Die Bereitschaft, sich als weiße Person mit Rassismus zu beschäftigen, ist für Latifa T. eine Voraussetzung dafür, dass eine Freundschaft und eine private und berufliche Partnerschaft möglich wird und funktioniert. Latifa T. berichtet, dass ihre weiße Partnerin »mitkriegt «, dass ihr Rassismuserfahrungen aufgenötigt werden, dass sie »im Laden oder auch im Freundeskreis« mit Rassismus konfrontiert wird. Im Unterschied zu weißen Personen kann sie sich nicht entscheiden, ob sie sich dieser Erfahrung aussetzen will oder nicht. Der Begriff »mitkriegen« deutet darauf hin, dass weiße Personen aufgrund der Beobachtung rassistischer Situationen eine rassismuskritische Haltung entwickeln können.

Latifa T. versucht sich rassistischen Erfahrungen zu entziehen, indem sie sich räumlich/körperlich und thematisch/intellektuell auf Distanz und damit in Sicherheit bringt. Indem sie versucht, sich nicht allzu sehr Rassismus auszusetzen, eröffnet sie sich Handlungsmöglichkeiten: Sie kann Personen, die sich rassistisch äußern oder verhalten, den Zutritt verweigern bzw. des Raumes verweisen und so die Souveränität über ihren Laden und ihr Leben behalten bzw. zurückgewinnen. Sie kann weiße Personen zur Verantwortung ziehen, sich mit ihrer Verstrickung in rassistische Diskurse und Strukturen auseinanderzusetzen, sodass sie diejenigen sind, die sich Rassismus stellen müssen. Sie selbst gewinnt dadurch Raum und Zeit, um sich anderen Dingen zu widmen und eigenen Themen nachzugehen. Latifa T. entscheidet sich, auf welche Weise und zu welchem Zeitpunkt sie (re-)agieren möchte. Es obliegt ihrer Entscheidung, ihre limitierten Ressourcen, ihre Zeit und ihre Energie, ihre emotionalen und ihre intellektuellen Kapazitäten einzusetzen. Sie hat den Entschluss gefasst, sich Rassismus soweit wie möglich zu entziehen und stattdessen Räume der Erholung, der Selbstermächtigung und der alltäglichen Selbstverständlichkeit zu schaffen. Ihr Entschluss ist eine Antwort auf den antimuslimischen Rassismus: Sie unterwirft sich dem hegemonialen Diskurs, indem sie ihn in Rechnung stellen muss, gleichzeitig widersteht sie ihm, indem sie ihn in Schach hält und die Möglichkeiten, Deutungs- und Handlungsmacht zu ihren Gunsten auszuweiten, ergreift. Diese Möglichkeiten bieten sich 
ihr aufgrund des sozialen Raums, in dem sie sich bewegt: Er ist von kritischer Diversität und den Kämpfen sozialer Bewegungen geprägt.

Adem O. entwickelt eine ähnliche Strategie, sich zu schützen. Als muslimische, romani, männliche Person blickt er auf »nicht nur eine, sondern mehrere« Erfahrungen mit dem Sicherheitsdiskurs zurück. Seine »spezifische Strategi:e« besteht darin, sich dem antimuslimischen Sicherheitsdiskurs zu »entzieh[en]«, indem er sicherheitspolitische Themen aktiv zurückweist. Als muslimischer Rom wird er als Kronzeuge aufgerufen mit dem Ziel, ihn gegen andere Muslim:innen in Stellung $\mathrm{zu}$ bringen. Er verweigert diese Rolle in der Hoffnung, zumindest diesen Diskursstrang aufzulösen: "Also das ist eine ganz bewusste Abgrenzungsstrategie. Auch damit lege ich einen Diskurs trocken." Sein situiertes Erfahrungswissen und die antizipierte Rassismuserfahrung helfen ihm $\mathrm{zu}$ erkennen, so seine Ausführungen, dass sein Beitrag zu bestimmten Themen instrumentalisiert wird. Um dieser Instrumentalisierung $\mathrm{zu}$ entgehen und gewisse »Rollen nicht $\mathrm{zu}$ erfüllen«, entzieht er sich und reagiert nicht auf Anfragen, die er als antimuslimisch identifiziert. Adem O. sagt, dass es »keinen Sinn« ergibt, in der Dominanzgesellschaft auf muslimische Perspektiven aufmerksam zu machen, da die hegemoniale Anrufung als Sicherheitsbedrohung keine andere Positionierung zulässt und er nicht gehört wird. Er entzieht sich aber nicht gänzlich. Wenn er sich entscheidet, doch zu antworten, dann »erklärt « er zunächst die Spielregeln: »[D]as fing halt damit an, dass ich erklärt hab, äh es geht um Menschenrechte, das ist eigentlich das Thema."Er distanziert sich thematisch/intellektuell von der Diskursivierung des Islams als bedrohlich und verleiht seiner Thematisierung eine andere Rahmung. Er weist die fremdbestimmte Deutung und Kontrolle zurück und antwortet in einer Weise, zu der er sich selbst bemächtigt und die ihn ermächtigt. Inwiefern er von der Dominanzgesellschaft gehört wird, bleibt offen. Nach innen hin kann er in der muslimischen Rom-Community eigene Themen setzen und bearbeiten: »ä:hm haben wir auch gelernt äh, j:a: (.) äh eigene Schutzräume zu schaffen«. Das Verb »schaffen« deutet auf die 
selbstbestimmte, kreative Aktivität hin, im Unterschied zum passiven, reaktiven Antworten auf die Fragen Anderer. ${ }^{2}$

\section{"0k eigentlich muss ich hier weg"}

Ibtissam P. geht auf physische/körperliche Distanz und sucht einen räumlichen, in diesem Fall geografischen Abstand $\mathrm{zu}$ ihren Rassismuserfahrungen in Deutschland. Sie ist »müde« und »erschöpft«, fühlt sich »sehr, sehr stark eingeengt«: »Ok eigentlich muss ich hier weg.« Sie entscheidet sich, für mehrere Monate im Südwesten der USA zu leben. Die Sicherheitsdebatten dort und auch ihre intersektionalen Rassismuserfahrungen unterscheiden sich von jenen in Deutschland: »Und spannend war da, (.) ich hab mich die ganze wirklich bedroht gefühlt als Schwarze Frau.« Der unmittelbaren Bedrohung begegnet sie analytisch (»spannend«) und kann so die Angst durch intellektuelle Distanz bändigen. Ihre gewohnte Rassismuserfahrung als Hijabi in Deutschland tritt in den USA hinter jene als Schwarze Frau zurück. In Südspanien wiederum, wo sie ebenfalls mehrere Monate lang versucht, sich vom deutschen Rassismus zu erholen, tritt ihre muslimische Markierung wieder in den Vordergrund, allerdings auf andere Weise als im deutschen Kontext:

»[D]er nationale Diskurs (.) zu Muslimen ist ja grade in Südspanien einfach $\mathrm{n}$ anderer ist, im Sinne von so auch so ner Anerkennu:ng. Wir haben ja islamische:: sowas wie ne islamische Geschichte, Südspanien ist verwurzelt mit ner spanisch-äh mit ner islamischen Ceschichte. Äh wie das dann ausgehandelt ist, ist dahin gestellt. «

Die >Verwurzelung « in der islamischen Geschichte ist ein Zugeständnis Südspaniens, es ist in der Architektur, der Kultur und anderen Materialisierungen offensichtlich und kann nicht geleugnet werden. Der nationale Diskurs in Südspanien ist ein anderer als in Deutschland, weil er eine islamische Geschichte berücksichtigen muss. Ibtissam P.

2 Auf geschützte Räume wird im Kapitel Ver_Handlungen im Abschnitt Ver_Antworten ausführlich eingegangen. 
relativiert zwar das positive Beispiel, indem sie hervorhebt, dass das Eingeständnis einer islamischen Geschichte Südspaniens nicht gleichbedeutend ist mit der Wertschätzung einer gemeinsamen Geschichte oder Kultur. Die Tatsache als solche kann aber nicht geleugnet werden, sodass sie dort als Schwarze Muslimin anders positioniert wird und sich anders positionieren kann als in Deutschland. Die Anerkennung der historischen Existenz des Islams auf südspanischem Boden ist für Ibtissam P. ausschlaggebend dafür, dass sie dort »das Gefühl [hat], dass ich da auf einmal anders leben konnte«. Sie fühlt sich frei, selbstverständliche Dinge selbstverständlich zu tun, Sport zu treiben, tanzen $\mathrm{zu}$ gehen und einen Malkurs zu belegen. Gleichwohl fühlt sie sich dort nicht heimisch: »ähm aber weil ich auch nicht aus Spanien bin und ich halt auch nicht so vertraut war mit diesen mit dem Kontext von Spanien .

Der geografische, räumliche, physische Abstand, der hier in zwei verschiedenen Kontexten ein nationaler ist, und die körperliche Erfahrung, außerhalb Deutschlands anders adressiert zu werden und sich selbst anders wahrzunehmen, ermöglichen ihr, neu auf ihre deutsche Erfahrung zu blicken: Es existiert eine Wirklichkeit außerhalb der ihr vertrauten, ihr Körper wird anderswo anders wahrgenommen und fühlt sich deswegen auch anders an. In den USA fühlt sie sich als Schwarze Frau verletzlich, sie bezieht sich im Interview auf die von der Polizei ausgehende Gewalt gegen Schwarze. Ihre Sicherheit als Schwarze steht dort zur Disposition. Ihr Umgang damit ist ein intellektueller, sie analysiert sich in der Situation und schafft so eine Distanz zwischen dem erlebenden, bedrohten Körper und dem analysierenden, ermächtigten Intellekt. In Spanien wiederum ist der nationale Diskurs ein anderer. Die >islamischen Wurzeln bieten ihr als Muslimin eine Grundlage, dort anders leben zu können als in den USA oder in Deutschland. Sie kann als Hijabi aufatmen und sich frei fühlen. Gleichzeitig fühlt sie sich dort fremd, sie ist in Deutschland sozialisiert. In Deutschland wird sie als Muslimin, zumal mit Kopftuch, als Gefahr adressiert und ihre - aus ihrer Sicht - fraglos gegebene Zugehörigkeit in Frage gestellt. Indem sie auf räumliche/körperliche Distanz zu ihrem Alltag geht, kann sie ihre Erfahrungen als historisch, national und intersektional akzentuierte 
reflektieren und auf analytische Distanz zu der Ursache ihrer Erschöpfung gehen. Das ermöglicht es ihr, Handlungsmöglichkeiten zu erproben, die über Reaktionen auf ihre Adressierung hinausgehen. Ihr eröffnen sich Freiräume, Dinge für sich selbst zu tun. Es handelt sich dabei um Aktivitäten (Sport, Tanzen, Malen), die einer Schwarzen Hijabi im deutschen Kontext nicht so ohne weiteres möglich sind, auf rassifizierende Kommentierungen muss sie gefasst sein.

Sich dem deutschen Kontext und den belastenden Diskursen zu entziehen, bedeutet für Ibtissam P., sich der auch an sie persönlich gerichteten Adressierung zu entziehen und sich nicht zu Salafismus und Terrorismus positionieren zu müssen. Die räumliche/körperliche Distanzierung und die vergleichende Reflexion ermöglichen es Ibtissam P., nach ihrer Rückkehr auch diskursiv und thematisch/intellektuell auf Distanz zu gehen. Sie fällt eine Entscheidung: Sie reagiert nicht mehr auf ihre Anrufung, da sie den hegemonialen Diskurs nicht verändern kann, und geht stattdessen »selbstbestimmt «ihren eigenen Interessen nach.

\section{"also irgendwie man versucht natürlich sich auch dann anders zu (.) verwi:rklichen«}

Rabea S. beendet ein Arbeitsverhältnis und geht auf thematisch/intellektuelle, räumlich/physische und emotionale Distanz $\mathrm{zu}$ einer Situation, die sie belastet. Sie berät muslimische Klient:innen in einer staatlichen Institution und bemerkt, dass sowohl sie selbst als auch ihre Klient:innen im Vergleich $\mathrm{zu}$ den nicht-muslimischen Berater:innen und Klient:innen ungerecht behandelt werden. Im Unterschied zu den anderen Beratungsverhältnissen ist der Datenschutz im muslimischmuslimischen Beratungsverhältnis eingeschränkt. Es wird von ihr erwartet, Informationen über ihre Klient:innen preiszugeben. Sie berichtet von verschiedenen Hürden, die ihr bei der Ausübung ihrer Tätigkeit in den Weg gelegt werden. Sie spricht im Interview den Staat direkt an und wirft ihm vor, Muslim:innen auszuspähen und zu 
vernachlässigen: »Wie willst DU mir glaubhaft machen, dass du für uns DA bist? $^{3}$

Rabea S. signalisiert, dass sie den Staat durchschaut hat und zieht ihn in die Verantwortung. Implizit fordert sie, dass die Ungleichbehandlung beendet wird und Muslim:innen nicht nur gleichgestellt, sondern auch beschützt und umsorgt werden. Diese Forderung kehrt den Sicherheitsdiskurs in mehrfacher Hinsicht um: Rabea S. ist diejenige, die ein Bedürfnis nach und Recht auf Sicherheit und Fürsorge hat, sie ist diejenige, die in der Position ist, zu fordern und sie ist es, die sich getäuscht fühlt und anklagt. Im Sicherheitsdiskurs ist sie in der Bringschuld und steht mit dem Rücken an der Wand. Nun tadelt sie und fordert ihre Rechte ein.

Sie wird im Interview laut, nimmt sich Zeit und überlegt, wie sie ihre Bedürfnisse und Erfahrungen prägnant in Worte fassen kann. Im Verlauf des Gesprächs artikuliert Rabea S. ihre eigenen Sicherheitsbedürfnisse und fordert, dass der Staat für alle Bürger:innen gleichermaßen Verantwortung zu übernehmen hat. Sie ist nicht länger bereit, aufgrund ihrer Rassifizierung Ungerechtigkeiten zu ertragen. Ihre diesbezügliche Kritik an ihrer Arbeitsstelle wird nicht gehört, ihr wird nicht "glaubhaft" gemacht, dass der Staat auch für sie da ist. Deswegen entscheidet sich Rabea S., das Arbeitsverhältnis zu beenden und sich umzuorientieren: "[I]rgendwie: geht's dann doch (.) also irgendwie man versucht natürlich sich auch dann anders zu (.) verwi:rklichen.« Sie hat sich entschieden zu gehen, sich also räumlich/physisch und damit auch intellektuell und emotional zu distanzieren, um sich selbst zu schützen und die Ungerechtigkeit nicht länger zu ertragen und mitzutragen. Rabea $S$. ist zuversichtlich, dass es »irgendwie« weitergeht und sie sich selbstverständlich (»natürlich«) verwirklichen möchte, dies aber auch auf andere Weise möglich ist. Wenn dies im Beruf oder in diesem Beruf nicht möglich ist, weil ihr die Umstände schaden, stehen ihr auch andere Wege offen. Sie entscheidet sich, die diskriminierende Situation zu verlassen und kündigt ihre Arbeitsstelle.

3 Hier klingt auch der Vorwurf der Täuschung an, der im Kapitel Ver_Handlungen im Abschnitt Ver_Antworten diskutiert wurde. 
Rabea S. spricht an anderer Stelle ganz allgemein über den antimuslimischen Rassismus. Im Anschluss an ihre rationalen und kontrollierten Überlegungen zur Bedeutung des Sicherheitsdiskurses für muslimische Communities geht sie auf ihre eigene Wahrnehmung und schließlich auf ihre Gefühle ein: »Also es is zwischen Wut und Trauer (.) pendelt das dann immer bei mir.« Sie reagiert emotional auf ihre rassifizierende Adressierung, die ihr unter anderem in der medialen Berichterstattung begegnet und die Muslim:innen unter Generalverdacht stellt. Sie versucht, ihre Emotionen zu kontrollieren. Sie hofft, dass sie gehört wird, wenn ihre emotionale Betroffenheit als Folge ihrer Rassismuserfahrungen anerkannt wird. Sie wirkt unsicher und findet nur schwer die richtigen Worte: »Und manchmal weiß ich nicht: Was soll ich mehr empfinden? Also das ist immer so g-emotional immer auch so Chaos äh, was es bei mir dann verursacht.« Rabea S. ist sich nicht sicher, ob sie ihre Rassismuserfahrungen richtig deutet, weil sie häufig erleben muss, dass sie geleugnet oder bagatellisiert werden. ${ }^{4}$ Das "Chaos« rührt möglicherweise daher, dass ihr signalisiert wird, dass ihre emotionalen Reaktionen (»Wut und Trauer«) unangemessen sind, vielleicht werden sie nicht ernst genommen.

Rabea S. will die Ursache für ihren emotionalen Aufruhr verstehen und kontrollieren, vielleicht auch ihre Empfindungen regulieren. Sie beobachtet und analysiert ihre Gefühle in der Hoffnung, sich nicht von ihnen beherrschen zu lassen. »Was soll ich mehr empfinden? « Die Frage wirkt wie der Ruf nach einer klaren Anweisung, um das Chaos zu beenden und Klarheit zu gewinnen. Der Widerspruch, der in der Anweisung (»soll«) zu einer emotionalen Reaktion (»empfinden«) liegt, drückt ihre Verzweiflung aus: Sie ist bereit, anders zu empfinden, wenn sie nur wüsste, wie. Sie sucht nach einer kognitiven Lösung ihrer emotionalen Verstrickung. Der intellektuelle Austausch über den Sicherheitsdiskurs ersetzt nicht den Austausch über die Emotionen, die er auslöst. Trotz fachlicher Expertise und argumentativer Sicherheit in ihrer Kritik am Diskurs bleibt die Auseinandersetzung auf der Gefühlsebene unabgeschlossen. Ihr stehen keine Gelegenheiten zur Verfügung, über ihre 
Emotionen $\mathrm{zu}$ reflektieren, ihr »emotional[es...] Chaos« $\mathrm{zu}$ verstehen und zu ordnen, einen Umgang mit ihrer »Wut und Trauer $\mathrm{zu}$ finden. Die Frage nach angemessenen Empfindungen ist mit der Frage nach entsprechenden Deutungen und Handlungen verknüpft. Ihre überwältigenden Gefühle zu klären und $\mathrm{zu}$ ordnen und so in eine analytische Distanz zu ihnen und zum verletzenden Auslöser zu gehen, um anschließend wieder zu sich zurückzufinden und sich zu zentrieren, kann als Voraussetzung für angemessene (Sprech-)Handlungen notwendig werden. Die Sicherheit, die ihr eine Ordnung auf emotionaler Ebene gibt, könnte zu ihrer Handlungsfähigkeit beitragen.

\section{"weil ich das einfach so lächerlich finde»}

Khadija R. findet sich in den Sicherheitsdebatten in Deutschland nicht wieder, will sich damit nicht beschäftigen und kann sie nicht wirklich ernst nehmen: »Ähm, ja ich kann das gar nicht so richtig wahrnehmen oder ähm (.) mach mir da überhaupt gar nicht so wirklich Gedanken, weil ich das einfach so lächerlich finde."Sie geht auf Distanz dazu und grenzt sich $a b$. Das hegemoniale Wissen über Muslim:innen nimmt Khadija R. als verzerrt und falsch wahr, sie findet es »ächerlich", es verdient keine weitere Beachtung. Der hegemonialen Perspektive stellt sie ihre eigene entgegen. Vor dem Hintergrund ihrer Erfahrungen und ihres Wissens über sich selbst kann sie die Diskursivierung muslimischer junger Frauen als Gefahr nicht nachvollziehen: „Weil ich mir nicht vorstellen kann, dass ein kleines Kopftuch ${ }^{\circ}$ mädchen ${ }^{\circ}$ äh auf der Straße eine Gefahr darstellen kann. «Sie lacht auf, als sie "Kopftuch ${ }^{\circ}$ mädchen $^{\circ}$ « sagt und unterstreicht, wie »ächerlich « die Vorstellung ist, dass sie und andere Hijabis eine Gefahr darstellen könnten. Indem sie den Namen, der ihr im öffentlichen Diskurs gegeben wird, annimmt, um ihn der Lächerlichkeit preiszugeben, übernimmt sie die Kontrolle über die Bedeutung des Wortes und nutzt es für ihre eigene Erzählung.

Khadija R. berichtet von verschiedenen Rassismuserfahrungen. Im Zuge eines Bewerbungsverfahrens muss sie von einem Gebäude in ein anderes wechseln und lässt ihre »Sachen« zurück. Der aufgebrachte Wachmann trägt ihr ihre Tasche am weit ausgestreckten Arm durch 
zwei Gebäude hinterher, da er das klingende Mobiltelefon darin für eine Bombe hält: "»Nicht dass hier gleich was hochgeht $\iota . «$ In einer anderen Situation drängelt sich an der Kasse ein Kunde vor. Sie versucht zunächst durch Vorfahren, dann durch Hüsteln auf sich aufmerksam zu machen, um ihn schließlich mit ihrem Rollstuhl leicht anzustupsen. Die Person holt, ohne sich umzudrehen, mit dem Fuß nach hinten aus und tritt sie weg. In der Schilderung gesteht sie ein, dass sie den sich vordrängelnden Mann hätte zur Rede stellen sollen. Sie entschuldigt sich damit, dass sie "nicht so die Person [ist], die dazu was sagen kann«. Sein Verhalten erlebt sie trotz ihres eigenen Fehlers als unangemessen aggressiv und gewaltvoll. Sie ringt bei der Schilderung nach Worten, wiederholt sich mehrfach und fragt: »Ok, war-warum? Äh aus welchem Grund? Warum gibt es so Menschen?« Sie ist fassungslos. Obwohl ihre Frage zunächst nach den Motiven fragt, kehrt sie in der Evaluation der Situationen zu sich zurück und konzentriert sich auf die Effekte. Sie ist emotional betroffen: "Und man hat sich einfach so schlecht dabei gefühlt. "Sie abstrahiert von ihrem eigenen Gefühl und ihrer Betroffenheit (»man«) sowie von der konkreten Person (»so Menschen«). Durch die Generalisierung gelingt ihr eine Distanzierung. Sie teilt ihre emotionale Betroffenheit mit anderen Personen in vergleichbaren Situationen.

Obwohl Khadija R. zu Beginn des Interviews zu verstehen gibt, dass sie den Sicherheitsdiskurs nicht ernst nehmen kann, geht aus den konkreten Schilderungen ihrer Erfahrungen und aus ihrer emotionalen Involviertheit hervor, dass dies nur bedingt gelingt: Sie bleibt vom antimuslimischen Rassismus nicht unberührt. Sie weigert sich jedoch, sich von diesen Erfahrungen überwältigen zu lassen: Sie verallgemeinert ihre emotionale Betroffenheit und nimmt ihnen durch die Distanzierung von sich als Person das Verletzungspotential. Auch rahmt sie die Fülle an Beispielen, die sie im 22-minütigen Interview vorträgt, durch eine Basiserzählung, nach der ihre »Erfahrungen bis jetzt eigentlich immer ganz positiv« waren. Ihre Schilderungen fallen sehr knapp aus. Sie führt sie lediglich an, um sie sogleich wieder zu verdrängen, sie will nicht darüber nachdenken. Indem sie sie dennoch anführt, zielt ihre Schilderung nicht darauf, sich als Opfer zu thematisieren. Vielmehr versucht sie zu 
verstehen, was ihr und anderen passiert, warum Menschen anderen Gewalt antun und wie sich das für die Opfer der Gewalt anfühlt. Zudem dienen die Beispiele dazu, auf die Eingangsfrage zurückzukommen, sie kontert: »Und sind wir jetzt die be-Bedrohung oder sind es andere Leute mit deren Gedanken?« Resümierend stellt sie fest, dass sie vermehrt Diskriminierung und Gewalt erfährt, seitdem sie ein Kopftuch trägt. Ihre Erfahrung, als »armes Mädchen im Rollstuhl« »nett und freundlich« behandelt zu werden, weicht ihrer Erfahrung, nun als gefährliche Hijabi verhandelt zu werden.

Khadija R. reflektiert, dass ihr Gefahr »auf der Straße« droht, in der Öffentlichkeit, im nicht-muslimischen Außen. Zuhause und in ihrer Community ist sie geschützt. Sie kehrt die hegemoniale Erzählung um, wonach Kopftuch tragende Frauen durch das muslimische Innen - die Familie, andere Muslim:innen, die Community und den Islam unterdrückt werden und das nicht-muslimische Außen sie rettet und emanzipiert. Vor ihrem eigenen Erfahrungshintergrund sind muslimische Frauen mit Hijab in der Öffentlichkeit bedroht, hier erleben sie Diskriminierung und Gewalt. Angesichts dessen ist die hegemoniale Diskursivierung von Hijabis besonders abwegig (»lächerlich«): »Weil ich mir nicht vorstellen kann, dass ein kleines Kopftuch ${ }^{\circ}$ mädchen ${ }^{\circ}$ äh auf der Straße eine Gefahr darstellen kann.« Sie spitzt den Widerspruch zu, indem sie die rassistische Bezeichnung (’kleines Kopftuchmädchen`) und das koloniale Narrativ (>muslimische Frauen retten $\triangleleft$ ) dem Bedrohungsszenario gegenüberstellt. Wenn Musliminnen Opfer (des Islams, der muslimischen Kultur und muslimischer Männer) sind, dann stellen sie keine Gefahr dar, sondern benötigen Schutz. Ihre Erfahrung lehrt sie aber, dass sie zwar schutzbedürftig ist - allerdings angesichts des antimuslimischen Rassismus. Khadija R. nimmt die Bezeichnung >kleines Kopftuchmädchen an, aber nicht, um sich selbst als solches zu positionieren, sondern um die Inkonsistenz und >Lächerlichkeit< des hegemonialen Diskurses aufzudecken. Sie spricht von Demokratie und Menschenrechten, appelliert an Religions- und Meinungsfreiheit, an Fairness und ein "gutes Klima«. Sie hält auch hier der Dominanzgesellschaft den Spiegel vor, wendet die antimuslimische Argumentation um 
und fordert sozialverträgliches Verhalten, demokratische Rechte und die Gewährleistung ihrer Sicherheit ein.

\section{Performative Interventionen}

Das Sprechen über antimuslimischen Rassismus lässt sich nicht auf eine einzelne Diskriminierungserfahrung reduzieren - im Gegenteil legen die Gesprächspartner:innen großen Wert darauf, das Thema von einzelnen Erfahrungen zu trennen und auf Diskursstrukturen zu verweisen, die sie selbst überblicken, verstehen und einordnen können. Themen, die an sie herangetragen werden, können sie verkomplizieren, Fragen ausweiten, eigene Sprechhandlungen analysieren und kontextualisieren. Unsere Gesprächspartner:innen bieten eine alternative Sichtweise auf ihre Lebensrealitäten abseits der etablierten Ordnung an. ${ }^{5}$

Unter Rückgriff auf die eigenen Erfahrungshorizonte sind sie in der Lage, ein widerständiges Bewusstsein zu artikulieren und der Diskriminierung mit distinkten, selbstdefinierten Standpunkten entgegenzutreten. Diese reichen von mimetischen Sprachhandlungen, bei denen hegemoniale Diskursfiguren angeeignet, umgedeutet und zum Zwecke der Unterbrechung des antimuslimischen Rassismus zurückgeworfen werden, über Strategien, an sie herangetragene Fragen zu sezieren, historisch-politisch zu kontextualisieren und Diskursräume auszudifferenzieren, bis hin $\mathrm{zu}$ aktivistischen, pädagogischen und zivilgesellschaftlichen Interventionen, bei denen hegemoniale Narrative irritiert und mit multiplen Erzählungen verkompliziert werden. Die Gesprächspartner:innen untergraben hegemoniale Sprechakte im antimuslimischen Bedrohungsszenario: Dessen Effekt, zum Schweigen zu bringen, $\mathrm{zu}$ führen und $\mathrm{zu}$ disziplinieren, scheitert bisweilen an performativanalytischen Protestformen, die sich am Diskurs selbst orientieren, an 
dessen Themen und Begrifflichkeiten anknüpfen, sie umdeuten, irritieren oder aneignen und verkomplizieren.

\section{»ich beg- benutz immer diese Fachbegriffe, das tut mir leid"}

Adem O. stellt direkt zum Interviewbeginn klar, dass er sich vollkommen bewusst ist darüber, »dass ich natürlich in einer bestimmten Art und Weise gele:sen werde«. Um sich als muslimisch markierter Rom vor Racial Profiling an öffentlichen Orten zu schützen, verfolgt er »eine ganz spezifische Strategie«. Wenn er auf Reisen geht, etwa am Flughafen, trägt er Anzug und Hemd. Den hegemonialen Sicherheitsdiskurs versteht er als »eine Art (2) Theater, äh in dem festgelegte Rolle sind«. Das bedeutet aber nicht, dass er den Zuschreibungen und Debatten ohnmächtig ausgeliefert ist. Er betritt die diskursive Bühne und spielt mit den Bedeutungen, er eignet sich sozioökonomische Codes an, um die rassifizierende Wahrnehmung zu irritieren oder zu neutralisieren. Schließlich werden sowohl Muslim:innen als auch Rom:nja mit einem bestimmten sozialen Habitus assoziiert, mit Armut und Mangel an Bildung und sozialem Kapital in Verbindung gebracht. Wenn Adem O. sich das Kostüm der bürgerlichen Mitte anzieht, also Anzug und Hemd trägt, entgeht er den rassistisch motivierten Kontrollen: "und ä:hm in der Regel werde ich dann auch nicht ${ }^{\circ}$ kontrolliert ${ }^{\circ}$." Adem O. senkt die Stimme, als befürchte er, seiner Strategie überführt zu werden, gleichzeitig amüsiert ihn die mimikryhafte Performanz, mit der er den Diskurs zu untergraben hofft. Er will nichts dem Zufall überlassen. Die bewusste, fast schon parodistische Nachahmung sozioökonomischer Symbole soll hier weniger affirmieren als vielmehr irritieren, Ausschlüsse entlarven und ihm Schutz bieten bzw. vor erneuten Rassismuserfahrungen bewahren. ${ }^{6}$

6 Homi K. Bhabha schreibt über Mimikry im kolonialen Setting: »Die metonymische Strategie produziert den Signifikanten der kolonialen Mimikry als den Affekt der Hybridität - als Modus sowohl der Aneignung als auch des Widerstandes, vom Disziplinierten zum Begehrenden. Als das diskriminierte Objekt wird das Metonym der Präsenz zur Stütze eines autoritären Voyeurismus und dient dazu, nur um so besser das Auge der Macht zur Schau zu stellen. Wenn 
Zum einen führt Adem O. praktisch vor, wie sich Rassismen miteinander und mit anderen sozialen Kategorien, darunter Klasse, aber auch Gender, vermengen und Hierarchien hervorbringen. Zum anderen zeigt er dadurch ihren performativen Charakter. Es handelt sich um keine gegebenen, schon gar nicht natürlich festgeschriebenen Identitäten, sondern um Konstrukte, die ihre Wirkmächtigkeit in ihrer wiederholten Performativität entfalten und gleichzeitig in der performativen Wiederholung verschieben lassen. Adem O. erlaubt sich einen spielerischen Umgang, um den Ausschluss an sich in seinen Funktionsweisen exemplarisch vorzuführen und die Trennlinien ins Absurde zu führen. Das heißt, er eignet sich die Zeichen der Autorität bewusst in jenen Situationen an, in denen er eigentlich diskriminiert werden soll - und wirft sie zurück. Die Subversion liegt darin, dass dualistische Ordnungen ausgehöhlt werden, was mitunter einen parodierenden Effekt hat. Im vorliegenden Fall liegt das Ziel der Strategie darin, in einer zeitlich begrenzten Situation der Unsicherheit bewusst sozioökonomische und -kulturelle Codes nachzuahmen, um sich vor (rassistischer) Diskriminierung zu schützen. Mehr nur als (weißbürgerliche) Autorität zu kopieren, irritiert die Handlung essentialistische Identitätskategorien und zieht die Ordnung ins Lächerliche.

Es ist nicht die einzige Umgangsweise, auf die Adem O. alltäglich zurückgreift: »Tatsächlich, das heißt das sind ganz ganz ähm ja alltägliche Strategien.«Die strategische Performativität tritt bereits im Sprechen über seine Erfahrung mit dem Sicherheitsdiskurs auf. Das performative Wissen, wie sich rassifizierte Subjekte im öffentlichen Raum $\mathrm{zu}$ geben haben, um sich Gewalt möglichst zu entziehen, spiegelt sich sprachlich wieder und strukturiert im Sprechen über die Erfahrung Handlungsoptionen. Indem Adem O. etwa durchgängig auf seine Wortwahl achtet und Fachtermini nutzt (»ich beg- benutz immer diese Fachbegriffe, das tut mir leid.«) und der wissenschaftlichen Befragung eine eigene wissenschaftliche Analyse und Expertise anbietet (»ich bin

dann die Diskriminierung in die Bestätigung des Hybriden umschlägt, wird das Signum der Autorität zu einer Maske, zu einer Farce.« (2000: 178). 
ja selbst Autodidakt« und »das ist meine Analyse«), expliziert er seine Sprecherposition und interveniert in das Geschehen.

Adem O. fordert Kontrolle über den Gesprächsverlauf und seine Wahrnehmung und Bewertung ein, indem er seine Position als Experte stärkt und die Deutungshoheit Anderer über sich abweist und zugleich seine Situation in einen größeren gesellschaftlichen Kontext setzt: »Also ich bin mir voll und ganz bewusst, dass unsere Gesellschaft von Rassismus, Antisemitismus und gruppenbezogener Menschenfeindlichkeit strukturie:rt ist. "Er wechselt im Sprechen mehrfach zwischen »wir« (Plural) und »ich« (Singular), setzt also seine eigenen Erfahrungen in den Zusammenhang kollektiver Erfahrungen: »Man muss natürlich sagen als Ro:ma haben wir andere historische nochmal Bedürfnisse als andere Muslime. U:nd ä:hm (.) und durch die historische Erfahrung haben wir auch gelernt (.) - ich spreche auch im- in einem wi:r.«

Damit greift Adem O. Fremdmarkierungen vor: Er analysiert und erklärt sein Sprechen und nimmt Einfluss auf die Beobachtung und die Analyse Anderer über ihn. Er reflektiert, wann er als Teil eines/welches Kollektivs spricht und wann als Einzelperson. Er liefert eine Analyse als Befragter und unterläuft die übliche Hierarchie zwischen Forscher:in und Beforschtem. ${ }^{7}$ Adem O. macht sich im Sprechen über seine Diskriminierungserfahrung selbst zum Untersuchungsgegenstand und signalisiert, dass er durchaus weiß, wer er ist und was er tut. Es könnte sein, dass ihm diese kurze Intervention auch deshalb wichtig ist, weil rassifizierten Menschen i.d.R. ein Expert:innentum abgesprochen wird: Es wird über sie gesprochen, ihr Erfahrungswissen als unwissenschaftliches, subjektives Wissen diskreditiert und abgewiesen. Einem hegemonialen Objektivitätsanspruch hält Adem O. ein »situiertes Wis-

7 Die Zurückweisung weißer Gadje-Wissenschaftler:innen gründet sicherlich auch auf der historischen Erfahrung und dem kollektiven Gedächtnis von Rom:nja und Sinti:zze. Lange vor dem Nationalsozialismus und bis in die Cegenwart hinein nehmen Wissenschaftler:innen eine maßgebliche Rolle in der Rassifizierung, Aussonderung, Verfolgung und Tötung von Rom:nja und Sinti:zze ein. 
sen ${ }^{8}$ entgegen, das den vermeintlich wissenschaftlichen Standpunkt Anderer, aus dem diese heraus sprechen, beobachten und bewerten, als subjektiv entlarvt und zugleich die eigene Position als ebenbürtig und relevant würdigt. Indem er sich selbst einer Gruppe zuordnet (»ich kollektiviere mich gerade«) kommt er einer Fremdzuschreibung zuvor.

Zugleich fungiert die analytische Intervention als vorgreifendes Korrektiv, um die Richtung der Bewertung und Betrachtung der Forschenden mitzubestimmen. Indem Adem O. sich im Sprechen über das eigene Sprechen stellt, davon abstrahiert und die Regeln seines Sprechens erläutert, weist er die wissenschaftliche Autorität der Forschenden über ihn zurück. Diese Strategie wendet er auch im Umgang mit dem Sicherheitsdiskurs im Allgemeinen und den öffentlichen Institutionen im Speziellen an. Adem O. meint, sich der Anrufung (Muslim/Rom/Bedrohung) entziehen zu können. Den Sicherheitsdiskurs kann er "glaub ich trocken legen, indem man eben diese Rolle nicht erfüllt. Und sich auch dieser Rolle entzieht«. Zwar werde er $\mathrm{zu}$ bestimmten Themen angefragt, entscheide aber selbst darüber, ob und wie er auf Adressierungen von außen reagiert: »[I]ch schaffe auch ein ganz bestimmtes Framing. Ich entziehe mich einem Framing und schaffe ein Framing. Ä:h und das heißt auch bestimmte Anfragen abzulehnen. « Adem O. ist sich seiner performativen Interventionsmöglichkeiten bewusst, mit denen er jene, die auf ihn blicken und ihn adressieren, mit dem eigenen (Zurück-)Blicken und der eigenen Themenwahl und -fokussierung konfrontiert und so das Geschehen mitbeeinflusst: »Ich belasse sie äh (.) in ihren Räumen. (.) Und indem ich mich nicht positioniere, müssen sie reflektieren, ob sie wollen oder nicht." Der hegemonialen Anrufung begegnet er mit eigenen Positionierungen und Expertisen: »[E]s geht um Menschenrechte, das ist eigentlich das Thema. Es geht nicht um Sinti und Roma und ich werde jetzt auch nicht sagen, erzählen, wie man über Sinti und Roma, was deren Kultur ist und ähm also ich werd jetzt da kein Roma-Flüsterer-Training anbieten oder so.«Adem O. schlägt hier eine

8 Haraway 1995: 80.

9 Diese Strategie wird im Abschnitt Schützende Distanzierungen interpretiert. 
Brücke: Er führt sowohl persönliche als auch fachliche Erfahrungen zusammen. Er blickt auf eigenes Wissen und eigene Kompetenzen, die im öffentlichen Diskurs zu wenig Beachtung finden, doch deren Relevanz er konsequent verteidigt.

\section{„WOAH, wo soll ich jetzt anfangen?»}

Ibtissam P. schenkt ihre volle Aufmerksamkeit den Fragen, die ihr angesichts ihrer Erfahrungen mit dem antimuslimischen Rassismus gestellt werden: »Ok, gut. Ich so: WOAH, wo soll ich jetzt anfangen? (.) Ähm, kannst du die Frage noch mal wiederholen. (10) Ähm (4)«. Statt unmittelbar zu antworten, lässt sie Fragen auf sich wirken, bittet um ihre Wiederholung und Klarstellung. Sie seziert die Fragen, die an sie herangetragen werden, und nähert sich Schritt für Schritt weiteren Fragestellungen und thematischen Querverbindungen, die für eine differenzierte Beantwortung notwendig erscheinen. "(4) also es geht aber auch schon auch so um hm also nicht nur jetzt heute im Moment oder aktuell, sondern auch so in der-(6)«. Ibtissam P. breitet das Diskussionsfeld aus, sie will das Geschehen zeitlich (»heute im Moment oder aktuell, sondern auch so in der-«), thematisch (»die mediale Berichterstattung zu Muslimen«) und örtlich (»in Deutschland«) präzisieren. Der jeweilige nationale Kontext, in dem Sicherheitsdebatten geführt werden, wie auch Ort und Zeit sind für Ibtissam P. zentrale Referenzpunkte, um die Mechanismen des antimuslimischen Bedrohungsszenarios einordnen $\mathrm{zu}$ können. ${ }^{10}$ Das besagte Thema setzt sie unmittelbar in einen globalen Zusammenhang: Sie spricht über 9/11 in den USA und die darauffolgenden Veränderungen im Umgang mit Muslim:innen, die auch Effekte auf Debatten und Praktiken in Europa und Deutschland hatten. 9/11 ist deshalb für sie relevant geworden, weil das Thema an sie herangetragen wurde. Retrospektiv macht Ibtissam P. den Mechanismus, bei dem ihr ein Thema aufgedrängt und zu ihrer Diskriminierung angeführt wird, selbst zum Thema ihrer Kritik. Das Thema »mediale Be-

10 Die nationalen Kontexte werden im Abschnitt Schützende Distanzierungen diskutiert. 
richterstattung zu Muslim:innen in Deutschland« im Zusammenhang mit 9/11 gibt gleichzeitig Auskunft über ihre Kindheitserfahrungen, also über jene Zeit, in der das Phänomen in ihr alltägliches Leben tritt und ihr suggeriert, dass »[ich] als Muslima (.) für das Land, in dem ich lebe, und die Welt, in der ich lebe, irgendwie f- eine Gefahr bin, so, ähm (.).«

Systematisch baut Ibtissam P. die eigene Erfahrung als Quellennachweis ein, um erst im Anschluss, nachdem die >richtigen $<$ und präzisen Fragen aufbereitet wurden, zu antworten. Sie hangelt sich zwischen Distanzierung und Annäherung an den Themen entlang und fordert eine Auffächerung in verschiedene Dimensionen, um dem großen Themenblock der Sicherheitsdebatten und ihrer Bedeutung für sie als rassifizierte Person gerecht zu werden. In der Subjektposition »Muslima« zu sein wird nach einer analytischen, retrospektiven Interpretation der medialen Repräsentation von Muslim:innen in Deutschland seit 9/11 für sie virulent. Ibtissam P. bildet damit einen strukturellen Rahmen, um in einem global-politischen Kontext, also über das Individuelle (»Muslima«/»Gefahr«) und über den deutschen Rahmen hinaus (Land/Welt), zu reflektieren. Das heißt, die wissenschaftlich-technische Eingangssequenz erfüllt den Zweck, eine Sprecherinposition zu etablieren und das eigene Sprechen möglich zu machen, um die eigene Erfahrung zu erzählen und wissenschaftlich zu untermauern.

Durch die analytische Schrittfolge kommt sie zu dem Ergebnis, dass Muslim:innen in Deutschland zu einer globalen Metapher für Gefahr werden, das heißt die mediale Berichterstattung nicht konkrete Menschen in den Blick nimmt, sondern auf Symbolen beruht, die transnational zirkulieren und auf Ereignisse woanders zurückgreifen, die sie lokal relevant machen. Die Gesprächspartnerin würdigt die persönlichen Erfahrungen in der Herausbildung ihrer eigenen Interpretation, stärkt kollektive Bezugspunkte und würdigt marginalisierte Positionen, sodass sie zugleich Wege alternativer, widerständiger Wissensproduktionen beschreitet. ${ }^{11}$ Einer stigmatisierenden und pauschalisierenden Medienlandschaft, die sich als objektiv und neutral definiert, hält sie 
eine eigene differenzierte Methode der Berichterstattung und Erörterung entgegen.

Sie demonstriert im Gesprächsverlauf, wie sie sich selbst gesellschaftlichen Themen annähert: Es bedarf der Selbstreflexion der Sprechenden und ihrer gesellschaftspolitischen Sprecherposition (Ich), der Explikation und Reflexion dessen, worum es geht (mediale Berichterstattung), wen diese wiederum berührt bzw. welches Thema (Markierung als Gefahr) und wo dies im Konkreten oder Allgemeinen örtlich stattfindet (Deutschland/Welt). Dadurch bringt Ibtissam P. gleichzeitig etwas anderes zum Ausdruck, nämlich dass die relevante Schnittstelle (9/11) gesellschaftlich relevant gemacht wurde und dadurch relevant für sie wird, das Thema also an sie herangetragen wird und keinesfalls selbst gewählt ist. Im Bedrohungsszenario haben Muslim:innen dem hegemonialen Interesse $\mathrm{zu}$ folgen und auf Fragen $\mathrm{zu}$ antworten. Das bedeutet auch, dass Muslim:innen, die sich mit diesen relevant gemachten Themen befassen, aus dem Blick verlieren, was eigentlich in ihrem eigenen Leben von Relevanz ist, also vom Eigenen abgelenkt werden. Die Gesprächspartnerin widersetzt sich einer solchen Ablenkung, indem sie einerseits auf das relevant Gemachte zurückgreift, um aber dann die eigene Erfahrung ins Feld zu führen und dadurch implizit gesellschaftspolitische Forderungen nach Anerkennung, Repräsentation, Zugehörigkeit und differenzierter, medialer Berichterstattung mit zu erzählen.

\section{"das war wirklich nicht meine Geschichte. Das ist nu:r ihre (.) Vorstellung und sie wollen das hö:ren«}

Jasmin V. positioniert sich als Geflüchtete, die in ihrer Sprache über ihre eigene Fluchterfahrung in weißen Räumen eingeschränkt wird. Sie beklagt, dass ihre Geschichte nicht gehört wird, sondern in dominanten Kontexten missgedeutet wird. Sie macht die Erfahrung, dass nur eine bestimmte Geschichte in der Dominanzgesellschaft über geflüchtete, (queere) Muslim:innen bzw. muslimische Trans* zugelassen ist. »Warum denken sie, wir sind Opfer?«, fragt Jasmin V. und weist unzutreffende Vorannahmen über ihren Fluchtgrund zurück. Es werde durchgän- 
gig vermutet, dass Jasmin V. wegen ihrer muslimischen Familie (aus einem mehrheitlich muslimischen Land) geflohen sei. Es gäbe bestimmte Bilder über »die muslimische Familie«. Jasmin V. beklagt, dass ihr ganz persönlicher Grund zur Flucht stereotyp interpretiert wird, wonach sie aufgrund familiärer Erfahrungen, das heißt der Verfolgung durch Familienmitglieder, nicht aber als regimekritische politische Aktivistin, geflohen sei. Gemäß einer solchen Vorstellung wird »die muslimische Familie« als Täter qua Religion und Kultur imaginiert, während unsichtbar gemacht wird, dass muslimische Familien auch in Deutschland leben und hiesigen Herrschaftsverhältnissen ausgesetzt sind: Die Dichotomie Hier Freiheit vs. Dort Unfreiheit geht nicht auf. Jasmin V. stellt klar: »[D]as war wirklich nicht meine Geschichte. Das ist nu:r ihre (.) Vorstellung und sie wOllen das hÖ:ren."

Jasmin V. ist mit einem hegemonialen Begehren nach einer queeren muslimischen Geflüchteten konfrontiert, die antimuslimische Stereotype bestätigt. Andere Geschichten können im öffentlichen Raum nicht gehört werden, nicht weil es keine Stimmen gibt, die widersprechen, sondern weil sie verdrängt und ausgeblendet werden. Jasmin V. wird auf ein stereotypes Opfer-Sein reduziert, ihre Erfahrungen zum Zwecke der Bestätigung antimuslimischer Narrative und stereotyper Darstellungen über mehrheitlich muslimische Länder instrumentalisiert. Sie beschreibt eine Abhängigkeitssituation: Um gesellschaftlich Zugänge zu erlangen, >Erlaubnis` zu bekommen, eintreten zu dürfen bzw. Teil der (Dominanz-)Gesellschaft zu sein, von ihr wahrgenommen und gehört zu werden, können Diskursregeln, die über das GehörtWerden entscheiden, nicht ignoriert werden. Jasmin V. beklagt: »Und wir müssen diese Geschichte w-(.) einfach wiederholen, a-auf ihre Interesse reden zum Beispiel für eine (.) als geflüchtete Person [...] (.) weiße Fantasie einfach wiederholen.« Muslimisierte Subjekte werden dazu gedrängt, die »Bilder«, »Vorstellungen« und »weißen Fantasien« $\mathrm{zu} »$ wiederholen«. Doch Jasmin V. weigert sich, in Argumentationen des interkulturellen Paradigmas zu sprechen und eine hegemoniale Geschichtsdeutung zu erzählen. Sie weist diese zurück, da sie nicht auf sie zutrifft. Die Gesprächspartnerin führt die persönliche Biografie als signifikante Wissensquelle für die Kritik sozialer Machtverhältnisse in 
Deutschland ein. Jasmin V. sucht nach Auswegen, um innerhalb der Diskursfelder, in die sie als Geflüchtete und queere Muslimin verwiesen und innerhalb der Kategorien, auf die sie durchgängig reduziert wird, den eigenen Geschichten Raum zu geben und zu widersprechen.

Das hindert Jasmin V. nicht daran, andere queere Fluchtgeschichten anzuerkennen, die ebenso ko-existieren können. Die narrative Intervention besteht darin, sensibel für verschiedene Erzählungen zu sein und multiplen Realitäten Gehör zu verschaffen. Umso wichtiger ist ihr der Begriff der Intersektionalität. Als Transfrau, als Muslimin und als Geflüchtete macht sie spezifische Erfahrungen. Es gibt demnach nicht die eine muslimische Erfahrung, sondern verschiedene, die sich überschneiden können. Jasmin V. lässt sich von bestimmten absoluten Begriffen weder vereinnahmen noch schränkt sie sich durch diese selbst ein. Die vielschichtige Erzählung über ihre Erfahrungen und Identitäten erfüllen zugleich den Zweck, politischen Forderungen nach Schutz Nachdruck zu verleihen und für jeweils spezifische Antworten zu plädieren. Jasmin V. braucht "mehr Sicherheit « gerade weil sie an der Schnittstelle verschiedener Diskriminierungskategorien existiert. Damit bringt sie auch zum Ausdruck, dass es wichtig ist, das Spezifische in jeder Rassismuserfahrung zu berücksichtigen. Die Unsicherheit, die aus der muslimischen Erfahrung hervorgeht, vermengt sich mit der Unsicherheit für Trans* of Color und Geflüchtete. Daraus folgen spezifische Sicherheitsbedürfnisse genauso wie Allianzvorhaben. Jasmin V.s narrative Intervention untergräbt das hegemoniale Islambild, reichert die Erfahrung als queere muslimische Geflüchtete an und weist damit Fantasien der Dominanzgesellschaft zurück.

\section{"Ä:hm is ja eine se:hr ((atmet tief aus)) breit gefasste Frage. (...) Die Debatten äh gibt=es (.) ja scho- seit etlichen Jahren«}

Merve Y. macht schon zu Beginn des Interviews klar, dass sie nicht als Betroffene sprechen will, sondern rein beruflich mit dem Thema zu tun hat und vor diesem Erfahrungshintergrund sprechen wird. Sie kommentiert Fragen im Gespräch (»Ä:hm is ja eine se:hr ((atmet tief aus)) breit gefasste Frage«) und betont: »Die Debatten äh gibt=es (.) ja scho- 
seit etlichen Jahren.« Merve Y. entscheidet sich dazu, das Thema Bedrohungsszenario "ganz allgemein« zu beantworten. Sie wahrt Anonymität und Professionalität. Sie berichtet nicht von persönlichen Rassismuserfahrungen, aber nicht weil sie keine hat. Indem sie auf die fachliche Diskussion setzt und sich der Diskurstermini bedient, macht sie sich die Regeln öffentlicher Debatten zunutze und verteidigt ihre Sprecherinposition als Expertin bzw. macht sich hörbar. ${ }^{12}$ Ihre strategische Überlegung geht davon aus, dass ihre fachliche Erfahrung nicht in Frage gestellt wird, wohl aber ihre persönliche. Die Entscheidung, als Expertin statt als Diskriminierte zu sprechen, gibt ihr Sicherheit im Gespräch. Merve Y. positioniert sich in einer Fachdebatte zum antimuslimischen Rassismus und bringt die Expertise anderer Kolleg:innen thematisch ein (»ich mein du hast darüber auch ne Veröffentlichung geschrieben«). Das ist eine Möglichkeit, an das Wissen Anderer anzuknüpfen, dieses zu bestätigen und auf eine politische Misslage hinzuweisen, in der die Bedürfnisse muslimischer Jugendlicher von Behörden und Ministerien übergangen, ausschließlich auf das Thema islamistischer Extremismus und Deradikalisierung beschränkt werden, und andere Themen, die muslimisches Leben betreffen, darunter auch Empowerment und Schutz, nicht förderwürdig erscheinen: »Weil das [Islamismus] nicht die Realität ist, mit der wir konfrontiert sind oder werden in unserer Arbeit. Dass es um ganz andere Themen geht. Und ä:h (.) auch vor allem um das Sicherheitsbedürfnis der rassifizierten Jugendlichen geht.«

Merve Y.s Einstieg "ganz allgemein« zu sprechen, kann gerade deshalb nicht als persönliche Einschätzung abgewiesen werden, weil auch andere "ganz allgemein« $\mathrm{zu}$ den gleichen Schlüssen gekommen sind. »Ganz allgemein« kann der antimuslimische Rassismus folglich nicht geleugnet werden, er ist nicht nur deshalb eine Realität, weil Betroffene davon berichten, sondern weil auch Wissenschaft und Praxis zu der gleichen Einschätzung kommen. Indirekt bestätigt Merve Y. damit auch die persönlichen Berichte anderer Muslim:innen als "ganz allgemein« wahr und zutreffend, weil sie im Einklang mit dem wissenschaft- 
lichen Diskurs stehen. Wie in den vorangegangen Ausführungen anderer Gesprächspartner:innen kommen auch in dieser Umgangsstrategie persönliche und wissenschaftliche/professionelle Erfahrungen zusammen, sie ergänzen sich gegenseitig. Merve Y. ist von diesen Entwicklungen nicht unberührt, sie flechtet, ähnlich wie andere Gesprächspartner:innen, eigene Gefühle ein. Es sei »immer wieder schmerzhaft«, dass öffentliche Diskussionen, die mit dem Bedrohungsszenario spielen, »hochkochen « und das Leben von Muslim:innen negativ beeinflussen. Sie führt die gesellschaftlichen Dynamiken an, wenn rassistische Vorurteile in den Medien zirkulieren und auch in den sozialen Medien aufgegriffen werden können, sodass Diskussionen um Muslim:innen und als solche Markierte »hochkochen«. Sie kritisiert, dass »rassistische Vorurteile sehr stark in den Medien präsent sind und (.) was dann auch vor allem auch in den sozialen Medien äh thematisiert wird«. Eine Lösung des Problems sieht sie darin, mit Jugendlichen gemeinsam zu reflektieren, wie Medien auf sie (ein-)wirken und wie Gegennarrative gestärkt werden können (»Und ich würde das [Sicherheitsdiskurse] gerne so ein bisschen um=umdeuten.«).

Merve Y.s Aussagen erinnern an Ibtissam P.s Kritik an etablierten Medien. Sie erkennt ein Potential in alternativen Medien gerade für Muslim:innen, die in etablierten Medien oft stereotyp dargestellt und übergangen werden. Das Gespräch bietet zugleich einen Anknüpfungspunkt zu Jasmin V.s Kritik, dass die dominante Mehrheit ihre Geschichte nicht hören will und sie dem öffentlichen Begehren ausgeliefert ist. Umso stärker erscheint Merve Y.s Argument, dass soziale Medien für benachteiligte Gruppen einen Handlungsspielraum ermöglichen, um ihre Realitäten in die Öffentlichkeit zu tragen und sich Gehör zu verschaffen.

\section{Globalhistorische Verflechtungen}

Einige unserer Gesprächspartner:innen sehen sich mit einer eurobzw. germanozentrischen Geschichtsschreibung und Erinnerungspolitik konfrontiert, in der Narrative und Erfahrungen ihrer Familie 
keinen Platz finden. Sie bleiben eine historische Randnotiz, werden umgedeutet und angepasst oder gar geleugnet und diskreditiert. Unsere Interviewpartner:innen heben globalhistorische Verknüpfungen hervor und rücken vor dem Hintergrund ihrer eigenen Erfahrungen und Bezüge außer- und innerhalb Europas Verbindungslinien in den Fokus. Sie finden unterschiedliche Auswege, um mit der historisch unterkomplexen Narration von Bürger:inschaft im Zusammenhang mit der unterfütterten Anrufung als Sicherheitsgefahr umzugehen. Sie schweigen in bestimmten Gesprächssituationen, zensieren sich selbst und schützen sich, weil sie die Erfahrung machen, dass ihnen ihre Geschichte genommen und ihre Erinnerung diskreditiert wird, dass ihre Lebensrealitäten geleugnet oder umgedeutet und sie auf das eine vermeintlich wahre Narrativ verpflichtet werden. Sie treffen Entscheidungen, wann und in welcher Form historische Adressierungen aufgegriffen und beantwortet werden, indem für sie relevante Bezüge aus dem kollektiven Gedächtnis in Erinnerung gerufen werden, sodass sie ihre eigenen Bedürfnisse im Licht der Vergangenheit und dem $\mathrm{Zu}$ sammenspiel globalhistorischer Verflechtungen artikulieren können. Sie lesen die nationale Geschichtsschreibung gegen den Strich und bringen verdeckte Ereignisse und Erinnerungen an die Oberfläche. Sie erinnern multidirektional, ergänzen und durchkreuzen das Feld der Erinnerung um weitere Dimensionen. Sie setzen die smuslimische Erfahrung ins Verhältnis zu anderen Ereignissen und Erfahrungen, changieren zwischen dem Universellen und dem Partikularen, zwischen der Zurückweisung eines einzigen nationalen Narrativs und dem Insistieren auf globalhistorischen Perspektivierungen und verschaffen sich dadurch Gehör für eigene Lebenswirklichkeiten und Interessen, Deutungen und Praktiken.

\section{"eine vollkommen andere, (.) äh als die Erfahrung, die man mir versucht sozusagen aufzudrücken"}

Adem O. identifiziert sich als muslimischer Rom, seine Rassismuserfahrungen beruhen auf beiden Rassismen und ihrer Intersektion. Der Präsenz muslimischer Rom:nja-Gemeinden in Deutschland wird in der 
breiten Öffentlichkeit wenig Beachtung geschenkt und sowohl in der Rassifizierung von Rom:nja als auch in jener von Muslim:innen wenig Rechnung getragen. Einerseits entlastet das Adem O., denn seine Unsichtbarkeit als Muslim erspart ihm diesbezüglich verletzende und unsinnige Fragen: »Und äh wir werden also nicht gefragt, wie wir zu Erdogan stehen ${ }^{\circ}$ oder $\mathrm{so}^{\circ}$, zum Beispiel. Und alle anderen werden gefragt.« Andererseits werden andere grenzüberschreitende und unsinnige Fragen an ihn gerichtet: »Wir werden eher gefragt, warum wir Muslime sind." Adem O. berichtet, dass seine Identifizierung als muslimischer Rom unsichtbar gemacht wird, indem sein gleichzeitiges Muslimischund Romsein in ein Muslimischsein und ein Romsein gespalten und gegeneinander ausgespielt wird. Er spricht von einem »weißen Diskurs«, in dem er als Rom gegen andere Muslim:innen instrumentalisiert werden soll: »Ok, das ist ein Roma, der ist bestimmt irgendwie unterdrückt von anderen Muslimen. "Mit der gesellschaftlichen Anrufung als Rom geht die Erwartungshaltung einher, eine Rolle einzunehmen, die der Dominanzgesellschaft im Umgang mit anderen Muslim:innen dient: »Und das ist ja die Rolle, die ich ausüben soll.«Indem Adem O. als (muslimischer) Rom angerufen und nach einer Diskriminierungs- und Unterdrückungserfahrung mit Islam und Muslim:innen befragt wird, wird er als Kronzeuge zu instrumentalisieren versucht. Er widersetzt sich jedoch der Teile-und-Herrsche-Strategie, die die Sorge um Rom:nja plakativ ins Feld führt, um Muslim:innen zu stigmatisieren: »Und das ist die Rolle, die ich eben nicht ausübe, weil tatsächlich für mich Muslimsein für meine eigene Biographie äh und (eher) etwas Emanzipatorisches bedeutet.«

Der diskursiven Spaltung Rom versus Muslim hält er eine widerständige Selbstpositionierung entgegen: Muslimischsein bedeutet für ihn weder ein Makel noch ein Defizit, vielmehr erlebt er es als Bereicherung und hebt dessen emanzipatorische Bedeutung hervor. "Muslimsein« stellt für ihn keine Gefahr dar, die von anderen Muslim:innen oder dem Islam ausgeht, sondern ist für ihn ein biographisch wichtiger Ankerpunkt, der ihm Schutz und Entwicklung bietet. Adem O. irritiert die homogene Zuschreibung von Muslim:innen als Täter:innen und Rom:nja als deren Opfer, indem er sich selbstbewusst als musli- 
mischer Rom präsentiert. Er untergräbt die eurozentrische Universalisierung des Unrechts an Europas Sinti:zze und Rom:nja, indem er die Geschichte muslimischer Rom:nja kontrapunktisch erzählt und globalhistorisch perspektiviert. Er weist die der Anrufung inhärente Narration zurück, wonach der Rassismus gegen Sinti:zze und Rom:nja der nationalsozialistischen Vergangenheit angehört, während andernorts und in anderen >Kulturen der geläuterten, bundesdeutschen Gesellschaft beschützt würden. Die deutsche Selbstvergewisserung, aus der Geschichte gelernt zu haben und nun Andere (wieder) zu führen und in Demokratie und Moderne einzuführen, beruft sich auf die Figur des Islams und muslimischer Herrschaft als europäisches Gegenbild.

Demgegenüber greift Adem O. auf eine Narration zurück, in der »ein idealisiertes Bild über das Osmanische Reich« vorherrscht. Das Bild entspricht zwar nicht der Realität, so Adem O., es ist »natürlich kein differenziertes Bild«. Gleichwohl erfüllt das »idealisierte Bild« wichtige Funktionen: Zum einen vermag es das Narrativ, der Erfahrung und der Identifizierung muslimischer Rom:nja einen Rahmen zu geben: »[E]ine Roma-Community im Balkan, die muslimisch ist und die sich unter Umständen idealisiert als Osmanen definieren."Adem O. liest die deutsch-europäische Geschichtserzählung kontrapunktisch und kann nun seine eigenen und die Erinnerungen seiner Community als muslimischer Rom emanzipatorisch zusammenführen. Zum Anderen entlarvt die Idealisierung jene Mechanismen, die Rom:nja gegen Muslim:innen instrumentalisieren, indem sie den Islam als rassistisch gegen Rom:nja in Stellung zu bringen versucht. Die Idealisierung ist jedoch »auch nicht differenziert«. Adem O.s Bezug auf das Narrativ geht es nicht darum, Geschichte $\mathrm{zu}$ idealisieren und Konflikte $\mathrm{zu}$ glätten. Vielmehr nutzt er das »idealisierte Bild«, um sich mit anderen Muslim:innen zu solidarisieren, und gleichzeitig die spezifische Erfahrung von (muslimischen) Rom:nja zu betonen: »Man muss natürlich sagen, als Roma haben wir andere historische nochmal Bedürfnisse als andere Muslime.«

Adem O. antwortet auf den Sicherheitsdiskurs, indem er einerseits sein (romani-)Muslimischsein emanzipatorisch deutet, widerständige 
Narrative in Erinnerung ruft und die Erfahrungen mit den Rassismen globalhistorisch verkompliziert. Andererseits kann er zwischen Romsein und Muslimsein changieren:»Tatsächlich haben Roma (.) ab einem bestimmten (2) ab einem bestimmten Level auch eine privilegierte Position. (.) Äh und es gibt ja eine Staatsräson.« Je nach Kontext wählt er bestimmte Aspekte aus und reklamiert ihre Relevanz, um sich so der einseitigen, instrumentalisierenden Anrufung zu widersetzen. Das bedeutet nicht, dass sich für Adem O. Muslimischsein und Romsein gegenseitig ausschließen, vielmehr legt er Wert auf das Zusammenspiel und die Komplexität verschiedener sozialer Verhältnisse, Erfahrungen und Erinnerungen. Schließlich ist seine Erfahrung »dann also eine vollkommen andere, (.) äh als die Erfahrung, die man mir versucht sozusagen aufzudrücken«.

\section{"konnte ich mich nicht so äußern wie ich (.) w- gerne, also ich (.) hab dann einfach oft dieses Thema vermieden (.) so"}

Ussama X. identifiziert sich als muslimisch und palästinensisch. Er ist in Deutschland geboren und aufgewachsen, die Flucht und die Vertreibung aus Palästina gehören zu zentralen Aspekten seiner Biografie. Während in seiner Familie darüber gesprochen wird, finden diese Erfahrungen in der deutschen Öffentlichkeit keinen Platz. Sie werden aus der postnazistischen Geschichtsschreibung der Bundesrepublik und der hegemonialen Narration verdrängt und/oder umgedeutet. Ussama X. findet sich regelmäßig in Gesprächssituationen wieder, in denen seine Erinnerungen und seine Erfahrungen nicht gehört werden und nicht zählen, sie werden als illegitim zurückgewiesen und er zum Schweigen gebracht: »[K]onnte ich mich nicht so äußern wie ich (.) wgerne, also ich (.) hab dann einfach oft dieses Thema vermieden (.) so.« Seine Erfahrung mit dem antimuslimischen Rassismus hängt unmittelbar mit der Auslöschung der palästinensischen Erfahrung aus der bundesdeutschen Geschichtsschreibung und dem hegemonialen deutschen, kollektiven Gedächtnis zusammen, der antimuslimische Rassismus zeigt sich ihm gleichzeitig antiarabisch und antipalästinensisch. Ussama X. wird wiederholt als Sicherheitsbedrohung angerufen, einer- 
seits im Verhältnis zur Dominanzgesellschaft, indem er als muslimisch adressiert wird, und andererseits im Verhältnis zur jüdischen Minderheit im In- und Ausland, indem er als muslimisch, arabisch und palästinensisch adressiert wird.

Bereits seit den 1970/80er Jahren sind die Namen >Palästinenser<, >Araber $<$ und >Terrorist $<$ im bundesdeutschen Diskurs eng verwoben und mit anderen Gewaltakten (etwa der RAF) diskursiv verkoppelt. Mithilfe des antimuslimischen Bedrohungsszenarios werden die historischen Karten neu gemischt und eine Anordnung skizziert, wonach das deutsche Wir, das (wieder aufblühende) jüdische Leben in Deutschland und der Staat Israel auf der gleichen Seite platziert werden, während ihnen gegenüber der muslimisch-arabisch-palästinensische Terrorist verortet wird. Gemeinsam mit >Juden « und >Jüdinnen < diskursivieren sich >Deutsche $<$ als Opfer von >Muslim:innen im Allgemeinen und im Besonderen als Opfer von >Arabern ner Sprachgemeinschaft nutzend, um die Vertreibung und Staatenlosigkeit der Palästinenser:innen zu de-thematisieren. Die Verschiebung der antisemitischen Gefahr auf Muslim:innen, Araber:innen und Palästinenser:innen, insbesondere auf solche, die als Migrant:innen und Geflüchtete von außen eindringen, beendet das kollektive sdeutscheく Täternarrativ und entlässt sich selbst aus der historischen Verantwortung für die Schoah und für die Vertreibung der nicht-jüdischen Palästinenser:innen. Gleichzeitig werden migrationsgesellschaftliche Defizite der Bundesrepublik in den >Nahen Osten v verschoben, sodass sich die Bundesrepublik auch dieser Verantwortung und ihrer (geo-)politischen Rolle entziehen bzw. sie verdecken kann.

Die palästinensische Erfahrung von Flucht und Vertreibung wird unsichtbar gemacht und mit ihrer Dethematisierung auch die deutsche, (post-)nazistische Verantwortung dafür. Dies kollidiert mit dem palästinensischen Narrativ und einer globalhistorischen Perspektivierung, wie sie Ussama X. reflektiert. Im Unterschied zur hegemonialen Diskursivierung stellt sich die >Naqba< seiner Familie weder als räumlich noch als zeitlich entferntes, wenn nicht gar fragwürdiges, Ereignis dar, sondern ist mit seinem Leben eng verwoben. In der deutschen Erinnerungskultur haben Ussama X.s Erfahrungen jedoch keinen Platz. 
Im Gegenteil sieht er sich mit einer Geschichtsschreibung und mit Narrativen konfrontiert, die palästinensische Erinnerungen als partikular, wenn nicht gar als falsch deuten, und sie der eigenen, als richtig und universell gültigen Erzählung, die als objektive Geschichtsschreibung präsentiert wird, entgegensetzen. Das hat Konsequenzen für Ussama $X$. und die (Un-)Möglichkeit, die eigene Biografie und die Vertreibung seiner Familie aus Palästina in alltäglichen Situationen anzusprechen. Er hat daraus gelernt und antizipiert eine erneute Zurechtweisung und einen diskursiven Ausschluss, indem er schweigt und sich nun selbst führt: "Ähm (.) und hab so mein Palästinenser (.) meine palästinensische Seite irgendwie so'n bisschen einfach so (.) so'n bisschen unter den Tisch oder so weißte so (.) nu::r nicht auffallen (.) oder so.«

Die Selbstführung bedeutet aber nicht, dass Ussama X. sich gänzlich dem Diskurs unterwirft und in einer passiven Position verharrt. Die Interviewsituation selbst ist exemplarisch für sein Vermögen, sich Gehör zu verschaffen. Er spricht sein Dilemma offen an und riskiert, sanktioniert zu werden, gleichzeitig erwartet er, von der ihn interviewenden Person verstanden zu werden. Ussama X. nutzt die einseitige Gesprächssituation - das Interview - und vertraut angesichts der Forschungsfrage auf die Bereitschaft der Forschenden zur Sensibilität für seine Selbstpositionierung und ihre Reflexion. In der Interviewsituation greift er auf konkurrierende, historische Gedächtnisse zurück, kontextualisiert nationale Geschichtsschreibungen globalhistorisch und führt vor Augen, dass seine biographische Erfahrung und kollektive Erinnerung mit der Anderer verwoben ist. Ussama X. Interesse daran, gehört zu werden, rührt auch daher, dass er nun als Vater besondere Verantwortung trägt: »Also ich hab jetzt auch zwei Jungs zum Beispiel ja? Und (1) vielleicht macht das auch $n$ Unterschied, weil ich mich mit diesem Thema beschäftige ja?«

Er ist nun selbst in der Position, das palästinensische Narrativ weiterzugeben, er trägt Verantwortung gegenüber seiner Community und seinen Kindern. ${ }^{13}$ Gleichzeitig lotet er Handlungsoptionen aus und sucht nach Wegen, Erinnerungen Rechnung zu tragen und Zeit 
und Energie nicht in ungeeigneten Situationen aufs Spiel zu setzen, in denen das Gegenüber keine Bereitschaft zeigt, die Engführung nationaler Erinnerungskultur zu hinterfragen und der Komplexität von Globalgeschichte und Widersprüchlichkeit von Narrativen Aufmerksamkeit zu schenken. Ussama X. reflektiert den Kontext und wählt wohlüberlegt aus: Er begreift das Erinnern und das Erzählen als individuelle und kollektive Ressourcen und entscheidet je nach Ort und Situation, wann er sie einsetzt und wann nicht. Die gleichzeitig aufgenötigte und selbstgewählte Zensur, der Rückzug aus Orten und Gesprächssituationen ist ein emanzipatorischer Akt, er bietet Schutz und Heilung und vermag so, die Erinnerung selbstbestimmt und eigenmächtig zu entfalten.

\section{"der nationale Diskurs (.) zu Muslimen [...] einfach $\mathrm{n}$ anderer ist, im Sinne von so auch so ner Anerkennu:ng“}

Ibtissam P. fasst ihre Erfahrung, als Muslimin in Deutschland zu leben, wie folgt zusammen: »Also dieses immer wieder hinterfragt werden. Frage von welche Berechtigung, welche Existenzberechtigung hat man?« Im Unterschied dazu blickt sie auf eine positive Auslandserfahrung in Südspanien zurück. ${ }^{14}$ Auch dort war sie »natürlich weiterhin eine Schwarze muslimische Frau«, jedoch konnte sie sich dort ganz anders im öffentlichen Raum bewegen und einen anderen Alltag führen. Schließlich ist »der nationale Diskurs (.) zu Muslimen [ist] ja grade in Südspanien einfach $\mathrm{n}$ anderer ist, im Sinne von so auch so ner Anerkenn:ung >Wir haben ja islamische:: sowas wie ne islamische Geschichte, Südspanien ist verwurzelt mit ner spanischäh mit ner islamischen Geschichte «« Die islamische Geschichte im heutigen Europa legt Zeugnis über die historisch-gewachsene Präsenz muslimischer Wirklichkeiten ab und prägt das Bewusstsein, sowohl jenes von (europäischen) Muslim:innen als auch jenes der Region. Zwar ist Geschichtsschreibung auch in Südspanien eine Frage der nationalen

14 Unter anderer Fragestellung wird diese Sequenz im Abschnitt Performative Interventionen rekonstruiert. 
Aushandlung und Rassismus kein Relikt der Vergangenheit, dessen ist sich Ibtissam P. bewusst. Nichtsdestotrotz liegt der Unterschied für sie darin, dass muslimische Geschichte in Südspanien anders als in Deutschland nicht geleugnet werden kann.

Ibtissam P. identifiziert sich weder als arabisch noch als amazighisch, sie verfügt auch nicht über persönliche, biografische Bezüge zu Südspanien oder dem nordafrikanischen Raum, in den Spaniens Muslim:innen einst deportiert wurden. Das hindert sie aber nicht daran, sich in der Geschichte Andalusiens wiederzufinden und auf das kollektive Gedächtnis als Muslimin zurückzugreifen. Darin kommt der acht Jahrhunderte währenden Präsenz von Muslim:innen im nördlichen Mittelmeerraum ein hoher erinnerungspolitischer Stellenwert zu. In Deutschland wird dieser historischen Präsenz kaum Rechnung getragen, ebensowenig wie den anderen Orten im heutigen Europa, in denen Muslim:innen heimisch waren und noch sind. Dem nationalen und europäischen Diskurs zufolge werden Muslim:innen außerhalb Europas verortet und als Gefahr für europäische Werte und Sicherheit konstruiert. Die nationale Geschichtsschreibung und Erinnerungskultur in Deutschland kommt ohne Muslim:innen aus bzw. führt sie als äußere Andere in die Diskussion ein. Durch die (zeitlich befristete) Verlagerung ihres Alltags nach Südspanien entzieht sich Ibtissam P. der hegemonialen, deutschen Adressierung als Andere, deren »Existenzberechtigung« auf dem Spiel steht. Durch ihre Selbstpositionierung (unter anderem) als Muslim:in kann sie im ehemaligen Al-Andalus ihre Anwesenheit als Selbstverständlichkeit einfordern und verteidigen. Dort verhält sie sich anders als in Deutschland, sie legt keine Rechenschaft über ihr So-Sein $a b$, sie bezieht keine Position (»Erklär mal Salafismus. Was- Wie stehst du dazu? Was machst du damit?«).

Indem Ibtissam P. auf die noch sichtbare muslimisch-arabischamazighische Präsenz in Südspanien verweist, kann sie die eigene Präsenz als Schwarze muslimische Hijabi als Teil der europäischen Geschichte und Gegenwart verteidigen, mehr noch: Sie kann ihren Platz dort reklamieren bzw. ganz selbstverständlich einnehmen. Sie kontextualisiert die eigene muslimische Erfahrung erinnerungspolitisch, rückt die islamische Geschichte innerhalb Europas ins Zentrum 
168 Muslimischsein im Sicherheitsdiskurs

und gibt jenen Perspektiven Raum, die in einer deutschen nationalen Erinnerungskultur zum Schweigen gebracht werden. 


\section{Zusammenfassung und Fazit}

In einem Zeitraum von insgesamt drei Jahren galt unser Forschungsinteresse den Erfahrungshorizonten von (als) Muslim:innen (Markierten), muslimischen Communities und Vertreter:innen aus Selbstorganisationen im Umgang mit dem hegemonialen Sicherheitsdiskurs in Deutschland. Wir fragten danach, wie sie auf die Anrufung als Sicherheitsgefahr antworten und interessierten uns vor allem dafür, wie es möglich ist, sich nicht gänzlich zu unterwerfen und eigene Interessen nicht aus dem Blick zu verlieren oder sie gar ins Spiel zu bringen: Wie navigieren Muslim:innen durch Strukturen des Othering und der Diskriminierung, von Ein- und Ausschlüssen, Stigmatisierung und Generalverdacht? Wie knüpfen sie an gesellschaftliche Debatten um Sicherheit an, wie beteiligen sie sich an Aushandlungsprozessen und wie positionieren sie sich, um sich Gehör für ihre Anliegen, Bedürfnisse und Erfahrungen zu verschaffen? Wie gelingt es (als) Muslim:innen (Markierten) am Diskurs teilzuhaben und dabei Definitions- und Handlungsmacht (wieder-) zu gewinnen?

Um die soziale Wirklichkeit von Muslim:innen und ihre Umgangsweisen mit dem Sicherheitsdiskurs zu rekonstruieren, bedienten wir uns zweier rekonstruktiver Methoden, die wir rassismus- und machtkritisch perspektivierten ${ }^{1}$. Ein rassismuskritischer und intersektionaler Ansatz erschien uns geboten, um - im Unterschied zur üblichen Essentialisierung und Homogenisierung von Muslim:innen - den vielstimmigen Deutungen Raum zu geben, die auf der Suche nach Strate- 
gien deren Vielfalt erst sichtbar werden lassen. Ein solcher Ansatz ist auch geboten, um Rassismus als Gesellschaft strukturierendes Machtverhältnis, das in den Wissensbeständen und Subjektivierungsweisen wirkt, im gesamten Forschungsprozess zu reflektieren und zu berücksichtigen. Den dominanzkulturellen Blick auf Muslim:innen in Rechnung stellend kehrten wir die Blickrichtung um, um muslimische Stimmen und den ihnen zugrunde liegenden Erfahrungen und Reflexionen zu hören.

Die Auswahl der Interviewpartner:innen und Diskussionsteilnehmer:innen ermöglichte es uns, eine große Bandbreite an Wissensbeständen $\mathrm{zu}$ vernehmen, die an der Schnittstelle von Alter, Gender, Sexualität, Klasse, Ability, Hautfarbe, Migration, Religion u.a. spezifisch adressiert werden. Mithilfe der Objektiven Hermeneutik ${ }^{2}$ rekonstruierten wir aus zehn Einzelinterviews Positionierungen und (Sprech-)Handlungen, während wir mithilfe der Dokumentarischen Methode $^{3}$ Aushandlungen zum Sicherheitsdiskurs in drei Gruppendiskussionen in den Blick nahmen. Die Reflexion beider Methoden in Hinblick auf ihre Methodologien und Anwendungsempfehlungen ermöglichte uns einerseits die Ausdifferenzierung von Wissensformen in hegemoniale und muslimisch/rassifizierte Wissensbestände. Andererseits konnten wir durch die Herausarbeitung der latenten Sinnstrukturen und konjunktiven Erfahrungsräume die unbewussten und die bewussten Umgangsweisen erschließen.

$\mathrm{Da}$ wir auch widerständiges (Sprach-)Handeln herausarbeiten wollten, war eine macht- und rassismuskritische Anpassung beider rekonstruktiver Forschungsmethoden nötig, um die Wissensbestände rassifizierter Personen interpretieren, analysieren und rekonstruieren zu können. Aus dem untersuchten empirischen Material ließ sich unter Berücksichtigung gouvernementalitätstheoretischer ${ }^{4}$ und rassismuskritischer Perspektiven ein Archiv an Handlungsoptionen

\footnotetext{
$2 \quad$ Wernet 2019.

3 Bohnsack 2018.

4 Foucault 2017a.
} 
nachvollziehen, mit denen Menschen mit antimuslimischer Rassismuserfahrung dem hegemonialen Sicherheitsdiskurs auf vielfältige, oft ambivalente und doch selbstermächtigende Weise begegnen.

\section{Zusammenfassung der Rekonstruktionsergebnisse}

Im Folgenden werden zunächst die Rekonstruktionsergebnisse aus den Gruppendiskussionen und Einzelinterviews vorgestellt, um anschließend allgemeine Schlussfolgerungen zu ziehen. Die vorgestellten und diskutierten Ergebnisse sind nicht repräsentativ für alle Muslim:innen und als solche Markierte. Vielmehr geben sie Aufschluss über unsere Rekonstruktion und Interpretation unterschiedlicher Deutungen und Umgangsweisen, Positionierungen und Verhandlungen, die sich unsere Gesprächspartner:innen in der Auseinandersetzung mit dem Sicherheitsdiskurs angeeignet haben und die sie in den Gesprächen mit uns und miteinander zur Sprache brachten.

\section{Ver_Handlungen}

In drei Gruppendiskussionen baten wir Muslim:innen, die sich entweder selbst als solche äußern oder als solche adressiert werden, miteinander darüber zu sprechen, wie sie den Sicherheitsdiskurs wahrnehmen, welche Auswirkungen er auf sie hat und wie sie damit umgehen. Im Gespräch mit anderen werden Reflexionsprozesse verbal zugänglich und in der Explikation und der Aushandlung von Positionen entwickelt und differenziert. Insofern boten sich Gruppendiskussionen zur Datenerhebung an.

Inter_Sektionen: Unsere Gesprächspartner:innen teilen die Erfahrung, mit kollektiven Verdächtigungen und Fragen nach ihrer Demokratiefähigkeit konfrontiert $\mathrm{zu}$ werden, sie werden alltäglich mit beleidigenden und verletzenden Begriffen als Fremde markiert und herabgewürdigt, in medialen und politischen Debatten über Sicherheit, Religion und Kultur als Bedrohung konturiert und kriminalisiert, in sicherheitspolitischer Adressierung beobachtet und 
reglementiert. Ihre Anrufung als >Muslim:innen jekte hervor ${ }^{5}$. Sie können sich dem nicht entziehen, haben aber die Möglichkeit, auf verschiedene Weisen $\mathrm{zu}$ antworten. Ihre Aussagen und Handlungen werden vor dem Hintergrund des Islam- und Sicherheitsdiskurses bewertet und reguliert. Unsere Gesprächspartner:innen antworten auf ihre Adressierung als Muslim:innen, vervielfältigen und verkomplizieren aber ihr Muslimischsein, indem sie es mit weiteren Positionierungen - Geschlecht, Sexualität, Klasse, Bildung, Migration, Kultur, Ethnizität, Sprache, Hautfarbe, Religion, Ability, Alter - kreuzen. So weisen sie einerseits die dem antimuslimischen Rassismus inhärente Essentialisierung und Homogenisierung >der Muslimin « und >des Muslim`zurück, greifen aber andererseits auf jene vergeschlechtlichten, sexualisierten, kolonialen, klassistischen Positionierungen zurück, die den antimuslimischen Rassismus im Allgemeinen und den Sicherheitsdiskurs im Besonderen durchziehen.

Ent_Solidarisierung: In allen Gruppendiskussionen wird auf das gesellschaftliche Misstrauen gegenüber Muslim:innen Bezug genommen. Der Sicherheitsdiskurs produziert ein Klima der Verdächtigung und Grenzziehung, das auch das Verhältnis innerhalb der Community berührt. Die Diskussionsteilnehmer:innen berichten davon, zu sicherheitsrelevanten Themen und Fragen Rede und Antwort stehen und sich von `bösen` Muslim:innen distanzieren und zu >liberalen`, ’konservativen zu müssen. Sie ringen um Differenzierung, handeln aus, mit wem es möglich ist, solidarisch zu sein und mit wem nicht, ohne sich selbst und die eigene Gemeinde allzu sehr in Gefahr zu bringen. So deutet sich in der Dynamik der Gespräche selbst eine gelebte Praxis der Solidarisierung und des Widerstands gegen allzu große Vereinnahmung durch die Dominanzgesellschaft an. Die Diskussionsteilnehmer:innen reflektieren Solidaritätsbekundungen, kommentieren und bewerten bisherige Erfahrungen und stellen Fragen an die Bedingungen und Chancen solidarischen Verhaltens. 
Ver_Antworten: In allen Gruppendiskussionen ziehen die Teilnehmer:innen den Staat zur Verantwortung und fordern ihn mehr oder weniger explizit auf, in demokratischen Prinzipien auch ihnen gegenüber gerecht zu werden. Sie sind sich der Anrufung bewusst, mit der sie dazu angehalten werden, ihren Beitrag zu Deradikalisierung und Prävention $\mathrm{zu}$ leisten. Sie wissen aus eigener Erfahrung, dass die Förderlandschaft auf islamistischen Extremismus fokussiert und finanzielle Anreize schafft mit dem Ziel, dass muslimische Gemeinden und Vereine die sicherheitspolitische Führung und Disziplinierung mittragen und sich selbst entsprechend führen. Sie kritisieren, auf die Rolle als Sicherheitspartner im Kampf gegen Islamismus reduziert zu werden, und dass nicht einmal in diesem Arbeitsfeld von einer Zusammenarbeit auf Augenhöhe die Rede sein kann. Stattdessen steht ihre Loyalität als Bürger:innen zur Disposition und sie werden als Muslim:innen unter Generalverdacht gestellt, sodass sie häufig mehr mit ihrer Verteidigung und Außenwahrnehmung beschäftigt sind als mit ihren community-internen Interessen und ihrem allgemeinen, gesellschaftlichen Engagement. Sie bemängeln die Intransparenz sicherheitspolitischer Apparate und Begriffsinstrumente und fordern, dass auch ihren Sicherheitsbedürfnissen und Schutzinteressen angesichts des antimuslimischen Rassismus Rechnung getragen wird.

\section{Selbst_Positionierungen}

In zehn Einzelinterviews baten wir Muslim:innen, die sich entweder selbst als solche zu Wort melden oder als solche adressiert werden, uns zu schildern, wie sie den Sicherheitsdiskurs wahrnehmen, welche Auswirkungen er auf sie hat und wie sie damit umgehen. Uns interessierte, wie sie sich angesichts ihrer Adressierung als >bedrohlich (und) fremd selbst positionieren. Um auch hier ein möglichst breites Feld an Deutungen und Praktiken zu erhalten, suchten wir unsere Gesprächspartner:innen nach ihrer Diversität aus.

Schützende Distanzierungen: Unsere Gesprächspartner:innen setzen - mehr oder weniger bewusst - verschiedene Strategien ein, um verletzenden Erfahrungen präventiv auszuweichen oder sich davon zu 
erholen. Sie gehen intellektuell auf Distanz, indem sie sich mit anderen Themen beschäftigen, sie gehen körperlich auf Distanz und wechseln die Arbeitsstelle oder den Wohnort und sie gehen emotional auf Distanz, indem sie ihre Erfahrungen verdrängen oder die überwältigenden Gefühle, die sie auslösen, zu bändigen versuchen. Sie kehren das Distanzierungsgebot gegenüber dem >islamistischen Terrorismus und >bösen Muslim:innen um, indem sie ihrerseits auf Distanz zum Bedrohungsszenario gehen. Sie erleben Rassismus als intellektuelle, physische und emotionale Herausforderung und versuchen ihr auf diesen Ebenen zu begegnen. Der Einschreibung in ihre Körper und Psychen versuchen sie durch befristete Migration, berufliche Entscheidungen und emotionale Strategien zu begegnen. Sie analysieren Rassismus in seiner Bedeutung für ihr Befinden und suchen nach Wegen, sich davon zu distanzieren, um sich in Sicherheit zu bringen.

Performative Interventionen: Unsere Gesprächspartner:innen ordnen einzelne Erfahrungen mit dem antimuslimischen Bedrohungsszenario in deren größeren gesellschaftlichen Zusammenhang ein und verweisen auf Diskursstrukturen, die sie selbst überblicken und verstehen. Sie verkomplizieren Themen, die an sie herangetragen werden, weiten Fragen aus, analysieren und kontextualisieren das eigene Sprechen. Der Diskriminierung treten sie mit selbstdefinierten Standpunkten vor dem Hintergrund eigener Erfahrungshorizonte, situierten Wissens und widerständigen Bewusstseins entgegen. Diese reichen von mimetischen Sprachhandlungen, bei denen hegemoniale Diskursfiguren angeeignet, umgedeutet und zum Zwecke der Unterbrechung des antimuslimischen Rassismus zurückgeworfen werden, über Strategien, an sie herangetragene Fragen zu sezieren, historisch-politisch zu kontextualisieren und Diskursräume auszudifferenzieren, bis hin zu aktivistischen, pädagogischen und zivilgesellschaftlichen Interventionen, bei denen hegemoniale Narrative irritiert und mit multiplen Erzählungen verkompliziert werden. Unsere Gesprächspartner:innen untergraben hegemoniale Sprechakte im antimuslimischen Bedrohungsszenario: Dessen Effekt, zum Schweigen zu bringen, zu führen und zu disziplinieren, scheitert zuweilen an performativ-analytischen Protest- 
formen, die hegemoniale Debatten umdeuten, irritieren und verkomplizieren.

Globalhistorische Verflechtungen: Unsere Interviewpartner:innen heben globalhistorische Verknüpfungen hervor und rücken vor dem Hintergrund ihrer eigenen oder familiären Erfahrungen, Narrative und Bezüge außer- und innerhalb Europas Verbindungslinien in den Fokus. Sie finden unterschiedliche Auswege, um mit der historisch unterkomplexen Narration von Bürger:inschaft im Zusammenhang mit der Anrufung als Sicherheitsgefahr umzugehen. Sie schweigen in bestimmten Gesprächssituationen, zensieren sich selbst und schützen sich, weil sie die Erfahrung machen, dass ihre Geschichte missrepräsentiert, umgedeutet und instrumentalisiert und ihre Erinnerung diskreditiert wird sowie ihre Lebensrealitäten geleugnet oder umgedeutet und sie auf das eine vermeintlich wahre Narrativ verpflichtet werden. Sie treffen Entscheidungen, wann und in welcher Form historische Adressierungen aufgegriffen und beantwortet werden, indem für sie relevante Bezüge aus dem kollektiven Gedächtnis in Erinnerung gerufen werden, sodass sie ihre eigenen Bedürfnisse im Licht der Vergangenheit und dem Zusammenspiel globalhistorischer Verflechtungen artikulieren können. Sie lesen die nationale Geschichtsschreibung gegen den Strich und bringen verdeckte Erinnerungen und Ereignisse an die Oberfläche. Sie erinnern multidirektional ${ }^{6}$, ergänzen und durchkreuzen das Feld der Erinnerung um weitere Dimensionen.

\section{Islambezogene Gouvernementalität: (Selbst-)Führung und Widerstand}

Im antimuslimischen Bedrohungsszenario tritt die Foucault'sche ${ }^{7}$ Gouvernementalität gerade dort in Erscheinung, wo weder ausschließlich Disziplin noch Strafe greifen, sondern vielmehr muslimische/muslimisierte Subjekte zur Selbstführung angerufen werden. Mit Blick

$6 \quad$ Rothberg 2009.

7 Foucault 2017a: 162. 
auf unsere Rekonstruktionsergebnisse lässt sich festhalten, dass (als) Muslim:innen (Markierte) die Erfahrung äußern, dass im hegemonialen Sicherheitsdiskurs Einfluss auf ihre Gewohnheiten und Gefühle, ihre Ängste, Erwartungen und Selbstbilder genommen wird und dass gesellschaftliche, strukturelle und institutionelle Rahmenbedingungen derart (um-)gestaltet werden, dass sich (als) Muslim:innen (Markierte) unter den hegemonialen Sicherheitsdiskurs (Salafismus, islamistischer Terrorismus, Deradikalisierung, Prävention, Leitkultur, Integration, Sicherheitspartnerschaft) $\mathrm{zu}$ fügen haben. Das bedeutet, dass in dieser neuen Regierungstechnologie Muslim:innen und als solche adressierte Menschen zusätzlich zu klassischen Machtapparaten, die kriminalisieren, pathologisieren, erziehen und ausschließen, als sehr spezifische Bevölkerungsgruppe angerufen und einbezogen werden, jedoch einseitig und instrumentell im staatlichen Interesse und vor dem Hintergrund orientalisierenden und rassifizierenden Wissens, mit dem Ziel, sie zu regulieren und Kontrolle zu dezentralisieren.

Doch Muslim:innen sind keine passiven Subjekte einer allgegenwärtigen Macht, die sie uneingeschränkt lenkt und kontrolliert, der sie sich ohnmächtig fügen oder gegenüber der sie unsichtbar bleiben. Auch muslimischen/muslimisierten Subjekten eröffnen sich durch die Unterwerfung unter den Sicherheitsdiskurs und den Namen, mit denen sie angerufen und adressiert werden, Handlungsspielräume ${ }^{8}$, sodass sie Freiheiten erweitern, eigene Positionierungen und Umgangsweisen inmitten des Spannungsverhältnisses von Macht- und Selbsttechniken selbstreflexiv, intersektional und in Ambivalenz statt Homogenität ${ }^{9}$ verhandeln.

Unsere Gesprächspartner:innen changieren zwischen verschiedenen gesellschaftlichen Positionierungen und bringen sie miteinander in Dialog. Sie berichten von (persönlichen) Rassismuserfahrungen im Bedrohungsszenario, setzen sie und den Sicherheitsdiskurs in einen politischen und historischen Zusammenhang und untermauern zugleich professionelle, wissenschaftliche und aktivistische Sprechpositionen.

$8 \quad$ Butler 2006.

9 Jain 2003; Wagenknecht 2003. 
Einige bemühen sich, antizipierten Rassismuserfahrungen vorzubeugen, zum Beispiel indem sie islamische oder als solche wahrgenommene Lektüre im öffentlichen Raum meiden, kein Arabisch sprechen oder auf deutsche Kulturgüter zurückgreifen. In der Erwartung, als Muslim:innen angesprochen und deshalb gemaßregelt oder benachteiligt zu werden, wird der öffentliche Rückgriff auf eigene Sprachen und Handlungen sowie die eigene Mobilität eingeschränkt. Das bedeutet nicht, dass unsere Gesprächspartner:innen sich den hegemonialen (Sprach-)Handlungen und den zugewiesenen Handlungsräumen vollkommen unterwerfen. Vielmehr eignen sie sich die Begrifflichkeiten subversiv an, deuten Themenfelder um, stellen Rückfragen und erweitern die Handlungsfelder um eigene Interessen, Perspektiven und Erfahrungen. Sie greifen auf performative Sprachcodes zurück, um Kontrolle über ihre Situation (wieder-) zu erlangen, indem sie Themenkomplexe sezieren und vom Gesagten abstrahieren, ihre Rassismuserfahrung problematisieren, zugleich die Situationen fachlich, wissenschaftlich und aktivistisch bewerten und untersuchen.

Den antimuslimischen Sprechakten begegnen sie mit performativen Interventionen und demonstrieren Handlungsoptionen bereits im Sprechen und Reflektieren über die Erfahrungen mit dem hegemonialen Sicherheitsdiskurs. Um sprechen zu können bzw. gehört zu werden, nehmen sie die Anrufung als Muslim:innen an, nicht aber um sie zu bestätigen, sondern um die Kategorie zu vervielfältigen und zu verkomplizieren und ihr Muslimischsein zu pluralisieren. Sie geben kollektiven wie individuellen Zuweisungen und Wirklichkeiten an der Schnittstelle von Alter, Gender, Sexualität, Klasse, Migration, Sprache, Hautfarbe, Ability und Religion Ausdruck, untergraben dadurch homogene Konstruktionen von Muslim:innen als Fremde und Andere, und stärken darüber hinaus eigene Wissensbestände, die in hegemonialen Islamund Sicherheitsdebatten marginalisiert werden.

Ingesamt wird deutlich, dass die geteilte Rassismuserfahrung Muslim:innen sowohl zu vereinen als auch zu trennen vermag. Gleichzeitig werden Selbstverständnisse und Lebensrealitäten mit anderen Erfahrungen ins Verhältnis gesetzt und zusammengeführt. In Gesprächssituationen teilen Muslim:innen verbindende und trennende Erfahrun- 
gen, sie geben einander Raum und verschaffen sich Gehör, knüpfen an eigene Erinnerungen und jene anderer aus ihren Familien oder Communities an, vervielfältigen sie oder reflektieren ausgehend davon eigene Positionierungen affirmativ oder kritisch. Sie stellen Fragen an das gesellschaftliche Zusammenleben in der Dominanzgesellschaft und unter Muslim:innen. Der Vorwurf der Illoyalität bereitet ihnen Sorgen: Sie kritisieren ein Klima der gegenseitigen Verdächtigungen und Beschuldigungen, entziehen sich Strategien des Teilens und Herrschens oder bedienen sich ihrer, und diskutieren darüber, wie eine Gesellschaft aussehen könnte, die den Bedürfnissen und Interessen aller Menschen gerecht wird, die ihre politische Teilhabe ermöglicht und CommunityStrukturen fördert und schützt, anstatt sie zu behindern, $\mathrm{zu}$ instrumentalisieren, zu stigmatisieren oder unter Generalverdacht zu stellen. Sie treten als zivilgesellschaftliche, wissenschaftliche, politische, kulturelle und soziale Akteur:innen auf, die Möglichkeiten der Solidarisierung diskutieren und Visionen austauschen für eine bessere, community-basierte Zusammenarbeit, in der sie nicht gegeneinander ausgespielt werden, sondern gemeinsame Räume gestalten, die ihnen Schutz bieten.

\section{Schlussfolgerung und Ausblick}

Den Umgang von (als) Muslim:innen (Markierten) mit dem antimuslimischen Rassismus empirisch $\mathrm{zu}$ untersuchen, legt einen methodenkritischen Perspektivenwechsel nahe. Die den Methoden zugrunde liegenden Theorien und auch ihre Anwendung müssen kritisch reflektiert und positivistische Grundannahmen aufgespürt werden, um ihren Einfluss auf den Forschungsprozess zu minimieren und der Analyse zugänglich zu machen. Hierzu sind rassismuskritische und intersektionale Zugänge in allen Phasen des Prozesses geboten: in der Formulierung der Forschungsfragestellung, im Zugang zum Feld, im Datenerhebungs- und Auswertungsprozess sowie in der Zusammensetzung des Forschungsteams und der Interpretationsgruppen. Methoden sind Werkzeuge, die machtkritisch eingesetzt auch der 
Annäherung an Wirklichkeiten und Perspektivierungen rassifizierter Menschen von Nutzen sein können. Die Reflexion grundlagentheoretischer und epistemologischer Positionen trägt zur Revision und Differenzierung von Forschungsmethoden bei.

Für unsere Studie war es notwendig, anstelle des hegemonialen >kommunikativen Wissens die sozialen Wirklichkeiten von Muslim:innen zum Ausgangspunkt der Rekonstruktion zu nehmen. Wir fragten danach, wie sie die (hegemoniale) Wirklichkeit als bedrohlich-muslimisch markierte Personen wahrnehmen, deuten und wie sie damit umgehen. Die Art und Weise, wie Muslim:innen in Forschungsvorhaben adressiert werden, welche Fragen an sie gerichtet werden, mit welchem Vokabular sie angerufen und welche Themen relevant gemacht oder in den Ausführungen der Interviewten von den Forscher:innen als relevant selektiert werden, bestimmt den weiteren Verlauf der Gespräche, der Interpretation und der abschließenden Auswertung, sodass sich durchaus auch in kritisch reflektierten Methoden stereotype Deutungsmuster wiederfinden, die bestätigt oder aufgegriffen werden.

In unserer Studie knüpfen die von uns befragten (als) Muslim:innen (Markierten) an ihrer begrifflichen und thematischen Adressierung an, weisen sie zurück oder greifen sie subversiv oder affirmativ auf, um gehört zu werden. Gerade Letzteres kann dazu führen, dass in Interviewsituationen (als) Muslim:innen (Markierte) in der Erwartung, ein bestimmtes Wissen zu präsentieren, hegemoniale Ordnungen reproduzieren oder sich an ihnen abarbeiten. In der Antizipation erneuter Rassismuserfahrungen sprechen sie in rassifizierenden Codes und unterwerfen sich - scheinbar, vorübergehend - dem Diskurs, ver-andern ihre Erfahrungen in Begriffen des hegemonialen, kommunikativen Wissens als kulturell oder religiös sfremd sie befragt, beobachtet und beforscht werden, handlungsfähig zu bleiben oder zu werden. Umso größer wirkt der Unterschied, wenn Muslim:innen sich in der Forschungssituation nicht erklären, rechtfertigen oder verteidigen und am stereotypen Wissen des Gegenübers abarbeiten müssen, sondern ein Gespräch vertiefen können, das an ihrem situierten Wissen ansetzt und nach differenzierenden Begriffen und einer Sprache sucht, mit der sie ihren Anliegen, Themen und Gefühlen 
Ausdruck verleihen können. Unsere Eingangsfrage ${ }^{10}$ eröffnete nicht nur durch die methodologische Gesprächsführung, sondern auch durch das Wissen, nicht missgedeutet, instrumentalisiert und vorgeführt zu werden, eine offene Gesprächsatmosphäre, die es möglich machte, auch sensible Themen anzusprechen und intime Perspektiven zu teilen.

Für gewöhnlich sorgt das Präfix »anti« im Begriff des antimuslimischen Rassismus für Irritation, richtet es sich doch auf den ersten Blick auf Handlungen und Diskurse, die Muslim:innen dämonisieren, ausgrenzen und kriminalisieren und sie zur Zielscheibe von Feindschaft und Gewalt machen. Eine solche Interpretation verliert aber jene Phänomene aus dem Blick, die (als) Muslim:innen (Markierte) exotisieren, paternalisieren oder - wie im Falle der Gouvernementalität - indirekt lenken, etwa indem sie als 'Sicherheitspartner angerufen und in das Othering aktiv eingebunden, zur >Leitkultur oder einem >deutschen Islam $\mathrm{zu}$ >besseren < deutschen Staatsbürger:innen zu erziehen, die islamische Religionspraxis nach christlichem Muster zu regulieren und das Verhältnis zwischen >guten und `bösen Muslim:innen weiter auszudifferenzieren. Dass auch jene Praktiken nicht im Widerspruch zur Funktionsweise des antimuslimischen Rassismus stehen, wurde in der vorliegenden empirischen Studie deutlich.

Antimuslimischer Rassismus wird als Wissensordnung wirksam, die >Muslim:innen als bedrohliche (und) fremde Andere adressiert, um ihre Ein- und Ausgrenzung zu rechtfertigen und die Barrieren und Maßnahmen, die sie an der fraglos gegebenen Zugehörigkeit hindern, $\mathrm{zu}$ legitimieren. Im Zuge dessen werden Muslim:innen als gesellschaftliche Subjekte hervorgebracht und bringen sich selbst als solche hervor. Ihre Rassifizierung hat sich freilich im Laufe der Jahrhunderte modifiziert, wie bereits Fanon ausführt. ${ }^{11}$ Im antimuslimischen Rassismus kulminieren >Religion<, >Kultur und >Herkunft< als Diskursstränge - in Intersektion mit weiteren Diskursen und Machtverhältnissen

10 Wir fragten danach, wie sie den Sicherheitsdiskurs wahrnehmen, welche Auswirkungen er auf sie hat und wie sie damit umgehen.

11 Fanon 1969: 39f. 
- und weisen >Muslim:innen $\mathrm{zu}$ - auch dann oder gerade dann, wenn sie im Inneren leben. Das konstitutive Außen wird nicht ausgeschlossen in dem Sinne, dass es entfernt wird und nicht mehr vorhanden ist, vielmehr muss es immer wieder neu nach Außen diskursiviert werden, also sichtbar bleiben als Ausgeschlossenes, sodass es das Innen weiterhin konstituieren kann. Mit Bezug auf Laclau schlägt Hall in Das verhängnisvolle Dreieck vor, zur Theoretisierung der anhaltenden Relevanz von Rassismus trotz der weit verbreiteten naturwissenschaftlichen Erkenntnis, dass es keine biologischen Rassen gibt, die Konstruktion von Rasse als "gleitenden Signifikanten « zu verstehen, der - zusammen mit Ethnizität und Nation - kulturelle Differenz legitimiert, die sich historisch und sozial entwickelt hat. Rasse, Ethnizität und Nation als Konzeptionen

»kulturelle[r] Differenz in diskursiven Termini neu zu denken [bedeutet,] Diskurs als dasjenige [zu] begreifen, was menschlichen Praktiken und Institutionen Bedeutung verleiht, was uns dazu fähig macht, die Welt zu verstehen, und folglich als das, was menschliche Praktiken zu bedeutungsvollen Praktiken macht, die genau deshalb geschichtlicher Natur sind, weil sie auf jene Weise signifizieren, auf die sie auch menschliche Differenzen markieren ${ }^{13}{ }^{13}$

Diese Theoretisierung von Rassismus unterscheidet sich von einer Deutung von Rassismus als Feindschaft oder Angst, wie sie in Begriffen der "Islamophobie« oder der »Islamfeindlichkeit« anklingen. Der antimuslimische Rassismus stellt freilich in der Geschichte des europäischen Rassismus keine Ausnahme dar, zeigt doch bereits Hall in Der Westen und der Rest ${ }^{14}$, wie zentral diskursive Aufspaltungen in sgute und >böse Andere im europäischen Expansions- und Kolonialprojekt auch in der Kolonisierung der Amerikas waren und eine intrinsische Funktion im Rassismus erfüllten. So gesehen stellt die Produktion sguter Fremder und die Bandbreite an kolonialen Strategien jenseits von Ausschluss

Laclau 2002: 67.

13 Hall 2017: 55.

14 Hall 1994. 
und Verfolgung keinen Widerspruch zum Rassismus dar, sondern eine zentrale Dimension insbesondere des postmodernen Rassismus, die rohe Formen physischer Gewalt und staatlichen Zwangs - als ultima ratio - erst denkbar macht und legitim erscheinen lässt.

Die >Anderen Macht und Herrschaft eingebunden, auch, aber nicht nur, durch Ausschluss. Wir halten daher einen Begriff des antimuslimischen Rassismus für sinnvoll, der den vormodernen und modernen Geschichten von Nationsbildung und Kolonialismus Rechnung trägt und die aktuellen Rückbezüge auf Religion, Kultur, Herkunft und Rasse (er-)neu(-t) verwebt. Es handelt sich um einen Begriff von (antimuslimischem) Rassismus, der die ambivalenten und widersprüchlichen Mechanismen nicht aus dem Blick verliert, deren Ziel und Methode es ist, nicht-weiße Menschen als Andere zu produzieren, zu disziplinieren, $\mathrm{zu}$ führen und einzuordnen. Der Begriff des Rassismus glättet die vielfältigen, gleichzeitig wirkenden und sich sowohl verstärkenden als auch irritierenden (diskursiven) Praktiken nicht zu einer einzigen Erzählung, in der Dämonisierung, Gewalt und Ausschluss als alleinige Mittel von Herrschaft und Zwang, Ausbeutung und Unterdrückung geltend gemacht werden. Rassismus dient auch und zentral der Selbstvergewisserung, sodass sowohl Aus- als auch Einschluss und sowohl »Islamophobie« als auch »Islamophilie $\ll^{15}$ dazugehören.

Im Prozess der Rassifizierung finden neben negativen durchaus romantisierende und exotisierende Vorstellungen Resonanz, die am Orientalismus anknüpfen und `Muslim:innen als Kontrastfolie zur Eigengruppe konstruieren. Ob >gut< oder >böse<, auch die zunehmenden Unter- und Zwischenkategorien halten an >Muslim:innen Figuren des ewigen Anderen fest, genauso wie an den Äquivalenz- und Differenzketten, wie Laclau und Hall sagen würden, und versuchen (als) Muslim:innen (Markierte) dazu zu bringen, von diesem Platz aus zu sprechen - oder ungehört zu bleiben.

Gleichwohl erlaubt dieses Verständnis von Rassismus (an-) zu erkennen, dass Muslim:innen auf antimuslimische Diskurse auch 
mit subversiven Gegen-Narrativen antworten, ihren Perspektiven und Stimmen widerständig Raum geben, sich vernetzen und verbünden, auch über die eigenen Communities hinaus, dass sie sich aktivistisch, künstlerisch, akademisch und zivilgesellschaftlich engagieren und mediale wie politische Debatten mitgestalten und eigenen Bedürfnissen und Interessen Gehör verschaffen. Die Performativität macht es möglich. Gleichzeitig reicht der Begriff des Widerstandes nicht aus, um der Spannbreite an Strategien Ausdruck zu verleihen, in denen Widerständiges, aber auch Affirmatives zur Geltung kommen und sowohl als das Eine als auch als das Andere gedeutet werden kann, in denen Handlungsoptionen also nicht eindeutig als Ausdruck entweder von Unterwerfung oder von Widerstand klassifiziert werden können. Vielmehr gibt es inmitten des Spektrums von Unterwerfung und Widerstand vielfältige, oft widersprüchliche Umgangsweisen, mit denen Muslim:innen innerhalb und außerhalb des diskursiven Rahmens das eigene Sprechen, die eigene Mobilität, das individuelle und kollektive Auftreten, die religiöse Praxis, das zivilgesellschaftliche Engagement, das professionelle Berufsleben und den Alltag (um-)deuten, Möglichkeiten und Grenzen austesten und so das Verhältnis von Disziplinierung und Selbstführung tagtäglich aushandeln. 



\section{Literaturverzeichnis}

Ahmed, Leila (1992): Women and Gender in Islam, New Haven \& London.

Ahmed, Sara (2004): Affective Economies. In: Social Text, 70 (22/2), S. 117-139.

Ahmed, Sara (2018): Das Glücksversprechen. Eine feministische Kulturkritik, Münster.

Al-Azmeh, Aziz (1996): Die Islamisierung des Islam. Imaginäre Welten einer politischen Theologie, Frankfurt a.M.

Amir-Moazami, Schirin (2014): Wer spricht wie für wen und warum? Zur Anerkennung, Authentizität und Repräsentation von Muslimen unter liberal-säkularen Bedingungen. In: Bertelsmann Stiftung (Hg.): Vielfältiges Deutschland. Bausteine für eine zukunftsfähige Gesellschaft, Gütersloh, S. 357-377.

Amir-Moazami, Schirin (2018) (Hg.): Der inspizierte Muslim. Zur Politisierung der Islamforschung in Europa, Bielefeld.

Amling, Steffen (2015): Peergroups und Zugehörigkeit. Empirische Rekonstruktionen und ungleichheitstheoretische Reflexionen, Wiesbaden.

Asad, Talal (2003): Formations of the Secular. Christianity, Islam, Modernity, Stanford.

Attia, Iman (2007): Orient- und IslamBilder. Interdisziplinäre Beiträge $\mathrm{zu}$ Orientalismus und antimuslimischem Rassismus, Münster.

Attia, Iman (2009): Die »westliche Kultur« und ihr Anderes. Zur Dekonstruktion von Orientalismus und antimuslimischem Rassismus, Bielefeld. 
Attia, Iman (2019a [2016]): Unzumutbare Koexistenz. Rassialisierungsprozesse von Muslimen und Musliminnen in historischer Perspektive. In: Uçar, Bülent/Kassis, Wassilis (Hg.): Antimuslimischer Rassismus und Islamfeindlichkeit, Göttingen, S. 125-140.

Attia, Iman (2019b): Die fremdgemachte Gewalt. Zum Verhältnis von antimuslimischem Rassismus, dem Bedrohungsszenario des >islamistischen Terrorismus` und Extremismusprävention, https://praeventionsnetzwerk.org/wp-content/uploads/2019/11 /Debattenbeitrag-I.-Attia-Die-fremdgemachte-Gewalt.pdf.

Attia, Iman/Bock, Andreas/Scherschel, Karin (Hg.) (2021 i.E.): Unter Beobachtung. Das Bedrohungsszenario des »islamistischen Terrorismus« aus den Perspektiven von Medien, Politik und muslimischen Communities, Bielefeld.

Attia, Iman/Keskinkılıç, Ozan Zakariya (2016): Antimuslimischer Rassismus. In: Mecheril, Paul (Hg.): Handbuch Migrationspädagogik, Weinheim \& Basel, S. 168-182.

Attia, Iman/Popal, Mariam (Hg.) (2018): BeDeutungen dekolonisieren. Spuren von (antimuslimischem) Rassismus, Münster.

Attia, Iman/Yılmaz-Günay, Koray (Hg.) (2021 i.E.): Antimuslimischer Rassismus im Kontext. Annäherungen - Relationen - Reflexionen, Berlin.

Austin, John (1975): How to do things with words, Cambridge.

Bäcker, Matthias (2020): Von der Gefahr zum »Gefährder«. In: Kulick, Andreas/Goldhammer, Michael (Hg.): Der Terrorist als Feind? Personalisierung im Polizei- und Völkerrecht, Tübingen, S. 147-165.

Balibar, Étienne (1992): Gibt es einen >Neorassismus? « In Ders./Wallerstein, Immanuel (Hg.): Rasse Klasse Nation. Ambivalente Identitäten, Hamburg, S. 23-38.

Bartel, Daniel/Liebscher, Doris/Remus, Juana (2017): Rassismus vor Gericht. Weiße Norm und Schwarzes Wissen im deutschen Recht. In: Fereidooni, Karim/El, Meral (Hg.), a.a.O., S. 361-383.

Bassiouni, Mahmoud (2014): Menschenrechte zwischen Universalität und islamischer Legitimität, Berlin.

Bayoumi, Moustafa (2009): How Does It Feel to Be a Problem? Being Young and Arab in America, New York. 
Bellmann, Uta (2011): >Orientierungen<. Über die Entstehung europäischer Bilder vom Orient und von Arabien in der Antike. Einflussfaktoren und stereotype Fortführungen im Mittelalter, Berlin.

Benz, Wolfgang (2012): Die Feinde aus dem Morgenland. Wie die Angst vor den Muslimen unsere Demokratie gefährdet, München.

Berger, Maurits S. (2014): A Brief History of Islam in Europe. Thirteen Centuries of Creed, Conflict and Coexistence, Leiden.

Berman, Nina (1996): Orientalismus, Kolonialismus und Moderne. Zum Bild des Orients in der deutschsprachigen Kultur um 1900, Stuttgart.

Bhabha, Homi K. (2000): Die Verortung der Kultur, Tübingen.

Bock, Andreas (2017): Islamistischer Terrorismus, die konstruierte Bedrohung. In: Zeitschrift für Außen- und Sicherheitspolitik (ZfAS), 10 (2), S. 245-265.

Bohnsack, Ralf (1989): Generation, Milieu und Geschlecht - Ergebnisse aus Gruppendiskussionen mit Jugendlichen, Opladen u.a.

Bohnsack, Ralf (2018): Dokumentarische Methode. In: Ders./Geimer, Alexander/Meuser, Michael (Hg.), a.a.O., S. 52-58.

Bohnsack, Ralf/Geimer, Alexander/Meuser, Michael (Hg.) (2018): Hauptbegriffe Qualitativer Sozialforschung, Opladen u.a.

Bohnsack, Ralf/Przyborksi, Aglaja (2010): Diskursorganisation, Gesprächsanalyse und die Methode der Gruppendiskussion. In: Ders./Dies./Schäffer, Burkhard (Hg.), a.a.O., S. 233-248.

Bohnsack, Ralf/Przyborski, Aglaja/Schäffer, Burkhard (Hg.) (2010): Das Gruppendiskussionsverfahren in der Forschungspraxis, Opladen u.a.

Brunner, Claudia (2011): Wissensobjekt Selbstmordattentat. Epistemische Gewalt und okzidentalistische Selbstvergewisserung in der Terrorismusforschung, Wiesbaden.

Brunnett, Regina/Gräfe, Stefanie (2003): Gouvernementalität und AntiTerror-Gesetze. Kritische Fragen an ein analytisches Konzept. In: Pieper, Marianne/Gutiérrez Rodríguez, Encarnación (Hg.), a.a.O., S. 50-67.

Bublitz, Hannelore (2014): Gouvernementalität, Normalisierung und Selbstführung. In: Vasilache, Andreas (Hg.): Gouvernementalität, 
Staat und Weltgesellschaft, Staat - Souveränität - Nation, Wiesbaden, S. 83-99.

Butler, Judith (1990): Gender Trouble. Feminism and the Subversion of Identity, New York.

Butler, Judith (2006): Haß spricht. Zur Politik des Performativen, Frankfurt a.M.

Collins, Patricia Hill (1986): Learning from the Outsider Within: The Sociological Significance of Black Feminist Thought, Social Problems, 33 (6), S. 14-32.

Collins, Patricia Hill (1993): Die gesellschaftliche Konstruktion Schwarzen feministischen Denkens. In: Joseph, Gloria (Hg.): Schwarzer Feminismus: Theorie und Politik afro-amerikanischer Frauen, Berlin, S. 17-52.

Cowans, Jon (Hg.) (2003): Early Modern Spain. A Documentary History, Philadelphia.

Cremer, Hendrik (2017): Rassismus? - Die Entscheidung des UNAusschusses gegen rassistische Diskriminierung (CERD) im »Fall Sarrazin.«In: Fereidooni, Karim/El, Meral (Hg.), a.a.O., S. 415-427.

Denkowski, Charles von (2014): Die formelle soziale Kontrolle des Jihadismus sowie der islamfeindlich motivierten Kriminalität - werden beide Phänomene in der Bundesrepublik Deutschland angemessen behandelt? In: Jahrbuch für Islamophobieforschung 2014, S. 42-65.

Denzin, Norman K./Lincoln, Yvonna S./Smith, Linda Tuhiwai (Hg.) (2008): Handbook of Critical and Indigenous Methodologies, Los Angeles u.a.

Dörner, Olaf/Loos, Peter/Schäffer, Burkhard/Schondelmayer, Anne (Hg.) (2019): Dokumentarische Methode: Triangulation und blinde Flecken, Opladen u.a.

Elahi, Farah/Khan, Omar (Hg.) (2017): Islamophobia. Still a challenge for us all, Runnymede Trust, London.

El-Qasem, Kawthar (2018): Palästina erzählen. Inversion als Strategie zur Bewahrung des eigenen in Dekulturalisierungsprozessen, Bielefeld.

Fanon, Frantz (1966 [1961]): Die Verdammten dieser Erde, Frankfurt a.M. 
Fanon, Frantz (1972 [1969]): Für eine afrikanische Revolution, Berlin.

Fanon, Frantz (2017 [1959]): Der Schleier, Wien.

Fereidooni, Karim/El, Meral (Hg.) (2017): Rassismuskritik und Widerstandsformen, Wiesbaden.

Flügel-Martinsen, Oliver (2014): Macht zwischen Unterwerfung und Widerstand. Zur Subjektkonstitution im politischen Denken Foucaults. In: Vasilache, Andreas (Hg.): Gouvernementalität, Staat und Weltgesellschaft. Studien zum Regieren im Anschluss an Foucault, Wiesbaden, S. 43-58.

Fouad, Hazim (2019): Zeitgenössische muslimische Kritik am Salafismus, Baden-Baden.

Foucault, Michel (1976): Die Macht und die Norm. In: Mikrophysik der Macht, Berlin, S. 114-123.

Foucault, Michel (1992): Was ist Kritik?, Berlin.

Foucault, Michel (2001 [1976]): In Verteidigung der Gesellschaft, Frankfurt a.M.

Foucault, Michel (2005): Heterotopien, Frankfurt a.M.

Foucault, Michel (2013a): Die Geburt der Biopolitik. In: Ders. (Hg.): Analytik der Macht, Frankfurt a.M., S. 180-187.

Foucault, Michel (2013b): Subjekt und Macht. In: Ders. (Hg.): Analytik der Macht, Frankfurt a.M., S. 240-263.

Foucault, Michel (2017a): Sicherheit, Territorium, Bevölkerung. Geschichte der Gouvernementalität I, Frankfurt a.M.

Foucault, Michel (2017b[1976]): Der Wille zum Wissen. Sexualität und Wahrheit 1, Frankfurt a.M.

Fuhrmann, Malte (2006): Der Traum vom deutschen Orient. Zwei deutsche Kolonien im Osmanischen Reich 1851-1918, Frankfurt a.M.

Graebsch, Christine (2019): Krimmigration: Die Verwobenheit strafrechtlicher mit migrationsrechtlicher Kontrolle unter besonderer Berücksichtigung des Pre-Crime-Rechts für »Gefährder.«In: KrimOJ, Kriminologie - Das Online-Journal, (1) 1, S. 75-103, https://do i.org/10.18716/ojs/krimoj/2019.1.6.

Gutiérrez Rodríguez, Encarnación (2003): Gouvernementalität und die Ethnisierung des Sozialen. Migration, Arbeit und Biopolitik. In: Pieper, Marianne/Dies. (Hg.), a.a.O., S. 161-178. 
Hafez, Kai/Schmidt, Sabrina (2014): Die Wahrnehmung des Islams in Deutschland. Religionsmonitor - verstehen was verbindet, Gütersloh.

Hall, Stuart (1994): Rassismus und kulturelle Identität, Hamburg.

Hall, Stuart (2004): Wer braucht >Identität? In: Ders. (Hg.): Ideologie, Identität, Repräsentation. Ausgewählte Schriften 4, Hamburg, S. 167-187.

Hall, Stuart (2017): Das verhängnisvolle Dreieck, Berlin.

Haraway, Donna (1995): Situiertes Wissen. Die Wissenschaftsfrage im Feminismus und das Privileg einer partialen Perspektive. In: Dies. (Hg.) (1995): Die Neuerfindung der Natur. Primaten, Cyborgs und Frauen, Frankfurt a.M., S. 73-97.

Hempfer, Klaus W./Volbers, Jörg (Hg.) (2011): Theorien des Performativen: Sprache - Wissen - Praxis. Eine kritische Bestandsaufnahme, Bielefeld.

Hering Torres, Max Sebastián (2006): Rassismus in der Vormoderne. Die »Reinheit des Blutes« im Spanien der Frühen Neuzeit, Frankfurt a.M.

Hernández Aguilar, Luis Manuel (2018): Die Orientalisierung des muslimischen Lebens in Deutschland. Die Deutsche Islamkonferenz und die Studie Muslimisches Leben in Deutschland. In: Attia, Iman/Popal, Mariam (Hg.), a.a.O., S. 297-319.

Höfert, Almut (2003): Den Feind beschreiben. »Türkengefahr« und europäisches Wissen über das Osmanische Reich 1450-1600, Frankfurt a.M.

Jain, Anil K. (2003): Die Spannung zwischen Subjekt und Politik und die Dialektik von Reflexion und Deflexion. In: Pieper, Marianne/Gutiérrez Rodríguez, Encarnación (Hg.), a.a.O., S. 240-256.

Johansen, Birgitte/Spielhaus, Riem (2012): Counting Deviance. Revisiting a Decade's Production of Surveys among Muslims in Western Europe, Journal of Muslims in Europe, 1, S. 81-112.

Johansen, Birgitte/Spielhaus, Riem (2018): Die Vermessung der Muslime. Ein Jahrzehnt quantitativer Forschung zu Muslimen in Westeuropa. In: Amir-Moazami, Schirin (Hg.), a.a.O., S. 125-157. 
Jukschat, Nadine/Leimbach, Katharina (2019): Radikalisierung als hegemoniales Paradigma. Eine empiriebasierte kritische Bestandsaufnahme. In: Behemoth. A Journal on Civilisation, 12 (2), S. 11-23.

Kabbani, Rana (1993): Mythos Morgenland. Wie Vorurteile und Klischees unser Bild vom Orient bis heute prägen, München.

Karakaşoğlu, Yasemin/Wojciechowicz, Anna Aleksandra (2017): Muslim_innen als Bedrohungsfigur für die Schule - Die Bedeutung des antimuslimischen Rassismus im pädagogischen Setting der Lehramtsausbildung. In: Fereidooni, Karim/El, Meral (Hg.), a.a.O., S. 507-528.

Katzer, Annette (2008): Araber in deutschen Augen. Das Araberbild der Deutschen vom 16. bis zum 19. Jahrhundert, Paderborn.

Keskinkılıç, Ozan Zakariya (2019a): Die Islamdebatte gehört zu Deutschland. Rechtspopulismus und antimuslimischer Rassismus im (post-)kolonialen Kontext, Berlin.

Keskinkılıç, Ozan Zakariya (2019b): Was ist antimuslimischer Rassismus?, https://www.bpb.de/politik/extremismus/radikalisierung spraevention/302514/was-ist-antimuslimischer-rassismus.

Keskinkılıç, Ozan Zakariya (2021 i.E.): Unbehagen, Sorge und Scham. Affekte in islambezogener Gouvernementalität und das Problem der Selbstzensur. In: Attia, Iman/Bock, Andreas/Scherschel, Karin (Hg.), a.a.O.

Keskinkılıç, Ozan Zakariya/Langer, Ármin (Hg.) (2018): Fremdgemacht $\&$ Reorientiert. Jüdisch-muslimische Verflechtungen, Berlin.

König, Ekkehard (2011): Bausteine einer allgemeinen Theorie des Performativen aus linguistischer Perspektive. In: Hempfer, Klaus W./Volbers, Jörg (Hg.), a.a.O., S. 43-68.

Laclau, Ernesto (2002 [1996]): Emanzipation und Differenz, Wien.

Lamnek, Siegfried/Krell, Claudia (2016): Qualitative Sozialforschung, Weinheim \& Basel.

Lemke, Thomas (2001): Gouvernementalität. In: Kleiner, Marcus S. (Hg.): Michel Foucault. Eine Einführung in sein Denken, Frankfurt a.M., S. 108-122. www.thomaslemkeweb.de/publikationen/Gouvernementalit \%E4t \%20_Kleiner-Sammelband_.pdf.

Lemke, Thomas (2007): Biopolitik zur Einführung, Hamburg. 
Lewicki, Aleksandra (2016): Islamophobia in Germany: National Report 2016. In: Bayrakli, Enes/Hafez, Farid (Hg.): European Islamophobia Report 2016, Istanbul, S. 213-236.

Lewis, Gail (1996): Situated Voices: >Black Women's Experience $<$ and Social Work. Feminist Review, 53, Speaking Out. Researching and Representing Women, S. 24-56.

Lohlker, Rüdiger (2014): Salafismus als Teil der Globalgeschichte. In: Schneiders, Thorsten G. (Hg.): Salafismus in Deutschland. Ursprünge und Gefahren einer islamistisch-fundamentalistischen Bewegung, Bielefeld, S. 137-148.

Mahmood, Saba (2015): Religious Difference in a Secular Age. A Minority Report, Princeton.

Marx, Daniela (2006): Vom `feministischen Schreckgespenstく zur gefragten Expertin. Alice Schwarzers Islamismuskritik als Eintrittskarte in die Welt der Mainstream-Medien. In: Jäger, Margret/Link, Jürgen (Hg.): Macht - Religion - Politik. Zur Renaissance religiöser Praktiken und Mentalitäten, Duisburg, S. 209-230.

Mecheril, Paul (1994): Die Lebenssituation Anderer Deutscher. Eine Annäherung in dreizehn thematischen Schritten. In: Ders./Teo, Thomas (Hg.): Andere Deutsche. Zur Lebenssituation von Menschen multiethnischer und multikultureller Herkunft, Berlin, S. 57-93.

Meuser, Michael (2018): Rekonstruktive Sozialforschung. In: Bohnsack, Ralf/Geimer, Alexander/Ders. (Hg.), a.a.O., S. 206-209.

Motadel, David (2017): Für Prophet und Führer. Die Islamische Welt und das Dritte Reich, Stuttgart.

Mühe, Nina (2019): Muslimische Religiosität als Stigma. Wie muslimische Schüler und Schülerinnen mit Stigmatisierung an den Schulen umgehen. In: Uçar, Bülent/Kassis, Wassilis (Hg.): Antimuslimischer Rassismus und Islamfeindlichkeit, Göttingen, S. 197-208.

Mustafa, Imad (2013): Der Politische Islam. Zwischen Muslimbrüdern, Hamas und Hizbollah, Wien.

Mustafa, Imad (2016): Die diskursive Konstruktion des »muslimischen Terrorismus« im Kontext »westlicher« Dominanzstrategien. In: DAS ARGUMENT, 319, S. 683-693. 
Naguib, Tarek (2013): Wenn der Antirassismus staatlich sanktioniert wird. In: recht - Zeitschrift für juristische Weiterbildung und Praxis, S. 13-27.

Nohl, Arnd-Michael (2013): Relationale Typenbildung und Mehrebenenvergleich. Neue Wege der Dokumentarischen Methode, Wiesbaden.

Nohl, Arnd-Michael (2017): Interview und Dokumentarische Methode. Anleitungen für die Forschungspraxis, Wiesbaden.

Nohl, Arnd-Michael/Schäffer, Burkhard/Loos, Peter/Przyborski, Aglaja (2013): Einleitung: Zur Entwicklung der Dokumentarischen Methode durch Ralf Bohnsack. In: Dies. (Hg.): Dokumentarische Methode. Grundlagen - Entwicklungen - Anwendungen, Opladen u.a., S. 9-40.

Núñez Muley, Francisco (1567): A Memorandum for the President of the Royal Audiencia and Chancery Court of the City and Kingdom of Granada. 2007 herausgegeben und übersetzt von Vincent Barletta, Chicago.

Oevermann, Ulrich (1993): Die Objektive Hermeneutik als unverzichtbare methodologische Grundlage für die Analyse von Subjektivität. Zugleich eine Kritik der Tiefenhermeneutik. In: Jung, Thomas/Müller-Doohm, Stefan (Hg.): »Wirklichkeit« im Deutungsprozeß: Verstehen und Methoden in den Kultur- und Sozialwissenschaften. Frankfurt a.M., S. 106-189.

Pieper, Marianne/Gutiérrez Rodríguez, Encarnación (Hg.) (2003): Gouvernementalität: Ein sozialwissenschaftliches Konzept in Anschluss an Foucault, Frankfurt a.M.

Pinn, Irmgard/Wehner, Marlies (1995): EuroPhantasien. Die islamische Frau aus westlicher Sicht, Duisburg.

Puar, Jasbir K. (2007): Terrorist Assemblages. Homonationalism in Queer Times, Durham \& London.

Qasem, Sindyan (2019): Erfahrungen von Rassismus als Radikalisierungsfaktor: Ein (Gegen-)Beispiel, https://www.bpb.de/politik/ext remismus/radikalisierungspraevention/295169/erfahrungen-von-r assismus-als-radikalisierungsfaktor-ein-gegen-beispiel. 
Rohde, Achim (2005): Der innere Orient. Orientalismus, Antisemitismus und Geschlecht im Deutschland des 18. bis 20. Jahrhunderts. In: Die Welt des Islams 45 (2), S. 370-411.

Rose, Nadine (2015): Subjekte der Macht bei Judith Butler und Michel Foucault. Machtvolle Diskurse, Subjektivierungen und Widerstand als Ausgangspunkt für eine rassismuskritische Perspektive in der Migrationsforschung. In: Reuter, Julia/Mecheril, Paul (Hg.): Schlüsselwerke der Migrationsforschung, Wiesbaden, S. 323-342.

Rothberg, Michael (2009): Multidirectional Memory. Remembering the Holocaust in the Age of Decolonization, California.

Said, Edward W. (1978): Orientalism, New York.

Said, Edward W. (1985): Orientalism reconsidered. In: Cultural Critique, 1, S. 89-107.

Said, Edward W. (1994): Culture and Imperialism, New York.

Sarasin, Philipp (2003): Zweierlei Rassismus? Die Selektion des Fremden als Problem in Michel Foucaults Verbindung von Biopolitik und Rassismus. In: Stingelin, Martin (Hg.): Biopolitik und Rassismus, Frankfurt a.M., S. 55-79.

Sarasin, Philipp (2005): Michel Foucault zur Einführung, Hamburg.

Scharathow, Wiebke (2014): Risiken des Widerstands. Jugendliche und ihre Rassismuserfahrungen, Bielefeld.

Scharathow, Wiebke (2017): Jugendliche und Rassismuserfahrungen. Kontexte, Handlungsherausforderungen und Umgangsweisen. In: Fereidooni, Karim/El, Meral (Hg.), a.a.O., S. 107-127.

Schiffauer, Werner (2020): Vorwurf des Islamismus. Warum das Konzept der Kontaktschuld problematisch ist, https://mediendienst-in tegration.de/fileadmin/Expertise_Kontaktschuld.pdf.

Schütz, Alfred (1974): Der sinnhafte Aufbau der sozialen Welt. Eine Einleitung in die verstehende Soziologie, Frankfurt a.M.

Shooman, Yasemin (2014): »... weil ihre Kultur so ist«. Narrative des antimuslimischen Rassismus, Bielefeld.

Shooman, Yasemin (2015): Zur Debatte über das Verhältnis von Antisemitismus, Rassismus und Islamfeindlichkeit. In: Rauschenberger, Katharina/Konitzer, Werner (Hg.): Antisemitismus und andere 
Feindseligkeiten. Interaktionen von Ressentiments, Frankfurt a.M., S. 125-156.

Soyer, François (2018): Glaube, Kultur und Angst. Antimuslimischer Rassismus im Spanien der Frühen Neuzeit und im Europa des 21. Jahrhunderts - ein Vergleich. In: Attia, Iman/Popal, Mariam (Hg.), a.a.O., S. 126-148.

Spielhaus, Riem (2011): Wer ist hier Muslim? Die Entwicklung eines islamischen Bewusstseins in Deutschland zwischen Selbstidentifikation und Fremdzuschreibung, Würzburg.

Spielhaus, Riem (2013): Muslime in der Statistik. Wer ist Muslim und wenn ja, wie viele?, https://mediendienst-integration.de/artikel/we r-ist-muslim-und-wenn-ja-wie-viele.html.

Stoler, Ann Laura (2002): Foucaults »Geschichte der Sexualität« und die koloniale Ordnung der Dinge. In: Conrad, Sebastian/Randeria, Shalini/Römhild, Regina (Hg.): Jenseits des Eurozentrismus. Postkoloniale Perspektiven in den Geschichts- und Kulturwissenschaften, Frankfurt a.M., S. 301-321.

Tamdgidi, Mohamed H. (2012): Beyond Islamophobia and Islamophilia as Western Epistemic Racisms. Revisiting Runnymede Trust's Definition in a World-History Context. Islamophobia Studies Journal, 1(1), S. 54-81. http://doi:10.13169/islastudj.1.1.0054.

Tezcan, Levent (2007): Kultur, Gouvernementalität der Religion und der Integrationsdiskurs. In: Wohlrab-Sahr, Monika/Ders. (Hg.): Konfliktfeld Islam in Europa, Baden-Baden, S. 51-74.

Wadud, Amina (1999): Qur'an and Woman. Rereading the Sacred Text from a Woman's Perspective, New York \& Oxford.

Wagenhofer, Sophie (2010): »Rassischer« Feind - politischer Freund? Inszenierung und Instrumentalisierung des Araberbildes im nationalsozialistischen Deutschland, Berlin.

Wagenknecht, Peter (2003): »Always be yourself!« Männlichkeit, Klassenposition und normative Heterosexualität in der Formierung von Subjektivität. In: Pieper, Marianne/Gutiérrez Rodríguez, Encarnación (Hg.), a.a.O., S. 196-223.

Wernet, Andreas (2009): Einführung in die Interpretationstechnik der Objektiven Hermeneutik, Wiesbaden. 
196 Muslimischsein im Sicherheitsdiskurs

Yeğenoğlu, Meyda (1998): Colonial Fantasies. Towards a feminist reading of Orientalism, New York. 


\section{Soziologie}

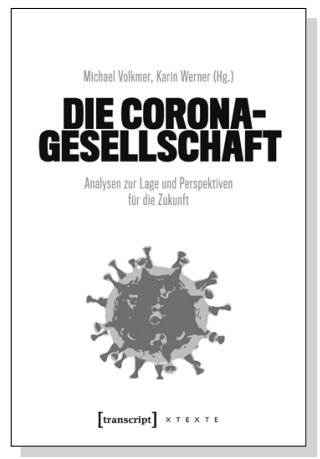

Michael Volkmer, Karin Werner (Hg.)

Die Corona-Gesellschaft

Analysen zur Lage und Perspektiven für die Zukunft

Juli 2020, 432 S., kart., 2 SW-Abbildungen

24,50€ (DE), 978-3-8376-5432-5

E-Book:

PDF: $21,99 €$ (DE), ISBN 978-3-8394-5432-9

EPUB: $21,99 €$ (DE), ISBN 978-3-7328-5432-5

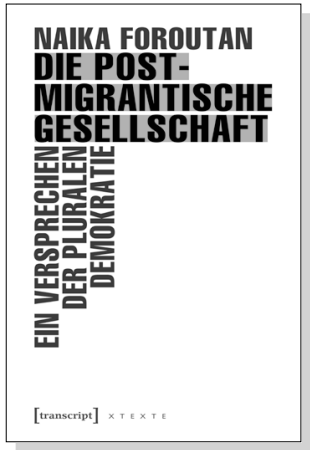

Naika Foroutan

Die postmigrantische Gesellschaft

Ein Versprechen der pluralen Demokratie

2019, 280 S., kart., 18 SW-Abbildungen

19,99€ (DE), 978-3-8376-4263-6

E-Book:

PDF: $17,99 €$ (DE), ISBN 978-3-8394-4263-0

EPUB: $17,99 €$ (DE), ISBN 978-3-7328-4263-6

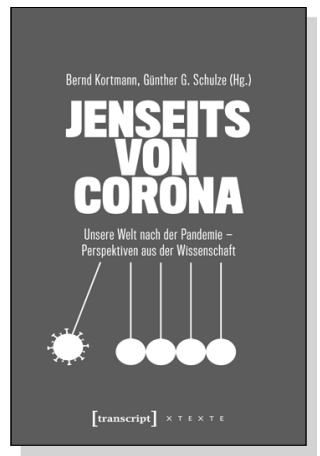

Bernd Kortmann, Günther G. Schulze (Hg.)

\section{Jenseits von Corona}

Unsere Welt nach der Pandemie -

Perspektiven aus der Wissenschaft

September 2020, 320 S., 1 SW-Abbildung

22,50€ (DE), 978-3-8376-5517-9

E-Book:

PDF: $19,99 €$ (DE), ISBN 978-3-8394-5517-3

EPUB: $19,99 €(D E)$, ISBN 978-3-7328-5517-9 


\section{Soziologie}

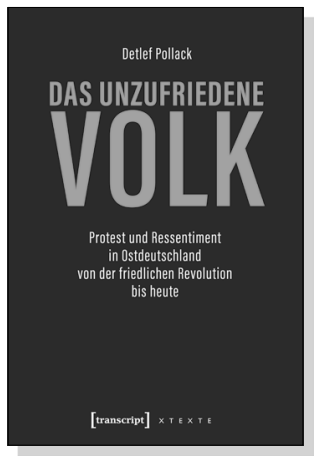

Detlef Pollack

\section{Das unzufriedene Volk}

Protest und Ressentiment in 0stdeutschland von der friedlichen Revolution bis heute

September 2020, 232 S., 6 SW-Abbildungen

20,00€ (DE), 978-3-8376-5238-3

E-Book:

PDF: $17,99 €(D E)$, ISBN 978-3-8394-5238-7

EPUB: $17,99 €(D E)$, ISBN 978-3-7328-5238-3

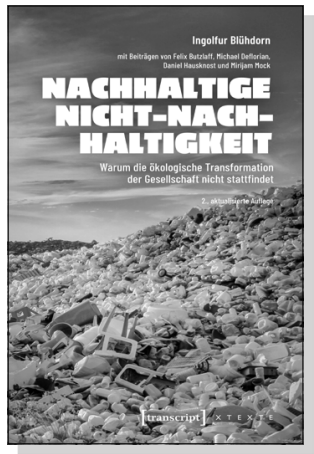

Ingolfur Blühdorn, Felix Butzlaff,

Michael Deflorian, Daniel Hausknost, Mirijam Mock

\section{Nachhaltige Nicht-Nachhaltigkeit}

Warum die ökologische Transformation der Gesellschaft nicht stattfindet

Juni 2020, 350 S., kart.

20,00€ (DE), 978-3-8376-5442-4

E-Book:

PDF: 17,99 € (DE), ISBN 978-3-8394-5442-8

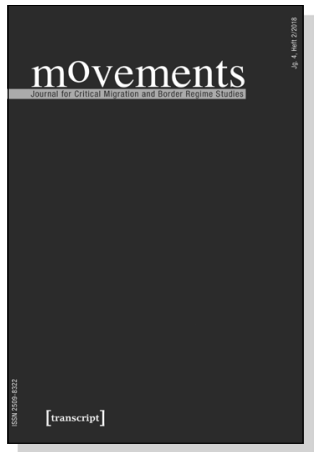

Juliane Karakayali, Bernd Kasparek (Hg.)

movements.

Journal for Critical Migration and Border Regime Studies

Jg. 4, Heft 2/2018

2019,246 S., kart.

$24,99 €(D E), 978-3-8376-4474-6$ 